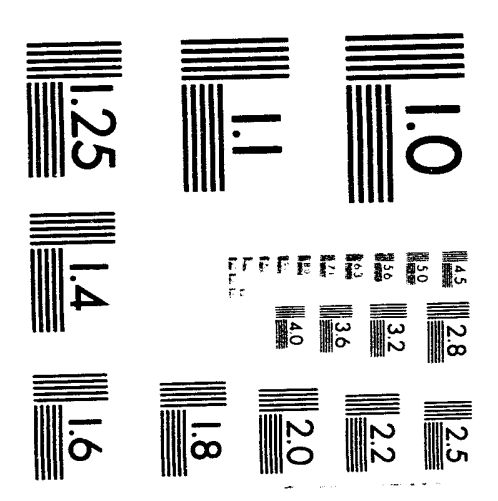




$$
\stackrel{\vec{o}}{\omega}
$$


LBL-34883

UC-404

\title{
Advances and Applications of Dynamic-Angle Spinning Nuclear Magnetic Resonance
}

\author{
Jay Harvey Baltisberger
}

Department of Chemistry

University of California

and

Materials Sciences Division

Lawrence Berkeley Laboratory

University of California

Berkeiey, California 94720

June 1993

\section{MASTER}

This work was supported by the Director, Office of Energy Research, Office of Basic Energy Sciences, Materials Sciences Division, of the U.S. Department of Energy under Contract No. DE-AC03-76SF00098. 
Advances and Applications of Dynamic-Angle Spinning Nuclear Magnetic Resonance Spectroscopy

Copyright $\odot 1993$

by

Jay Harvey Baltisberger

The Goverrment reserves for itself and others acting on its behalf a royalty free, nonexclusive, irrevocable, world-wide license for govermental purposes to publish, distribute, translate, duplicate, exhibit, and perform any such data copyrighted by the contractor. 


\title{
Advances and Applications of Dynamic-Angle Spinning \\ Nuclear Magnetic Resonance Spectroscopy
}

by

\author{
Jay Harvey Baltisberger \\ Doctor of Philosophy in Chemistry \\ University of California at Berkeley \\ Professor Alexander Pines, Chair
}

\begin{abstract}
This dissertation describes nuclear magnetic resonance experiments and theory which have been developed to study quadrupolar nuclei (those nuclei with spin greater than one-half) in the solid state. Primarily, the technique of dynamic-angle spinning is extensively reviewed and expanded upon in this thesis. Specifically, the improvement in both the resolution (two-dimensional pure-absorptive phase methods and DAS angle choice) and sensitivity (pulse-sequence development), along with effective spinning speed enhancement (again through choice of DA.S conditions or alternative multiple pulse schemes) of dynamic-angle spinning experiment were realized with both theory and experimental examples. The application of DAS to new types of nuclei (specifically the ${ }^{87} \mathrm{Rb}$ and ${ }^{85} \mathrm{Rb}$ nuclear spins) and materials (specifically amorphous solids) has also greatly expanded the possibilities of the use of DAS to study a larger range of materials. This dissertation is meant to demonstrate both recent advances and applications of the DAS technique and by no means represents a comprehensive study of any particular chemical problem.

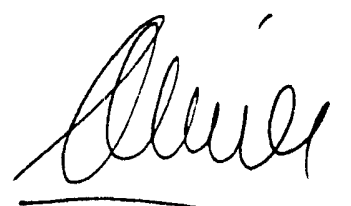


ciedicated to Julie, Mary and Richard 


\section{Table of Contents}

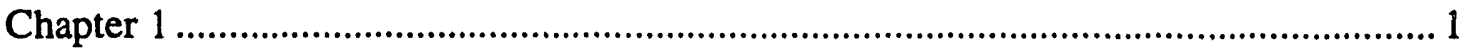

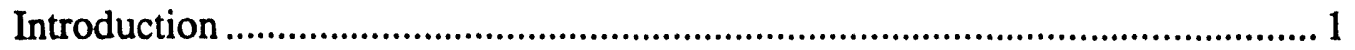

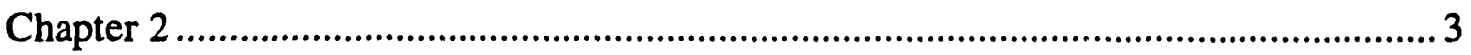

Theory of Nuclear Magnetic Resonance ....................................................... 3

Classical Magnetization Description................................................. 3

Quantum Mechanical Description...................................................5

Rotations and Tensors ................................................... 5

Perturbation Theory................................................................ 7

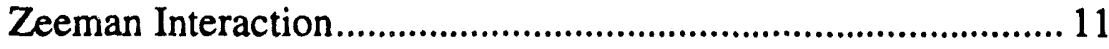

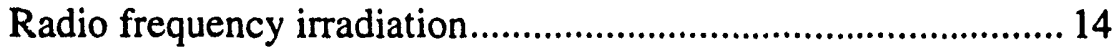

Chemical Shift Anisotropy ................................................... 15

Quadrupolar Interaction ..................................................... 17

Dipole-Dipole Interaction ..................................................... 21

Truncation of RF Hamiltonian by the Quadrupolar

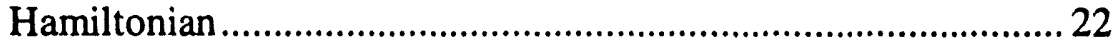

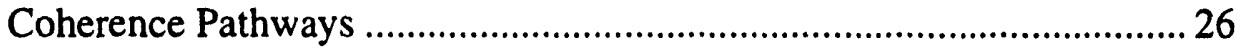

Eigenvalues from static samples ..................................................... 31

Eigenvalues from Rotating Samples ................................................. 34

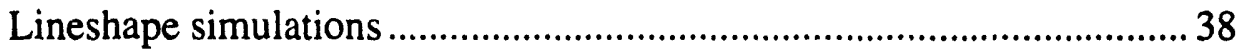

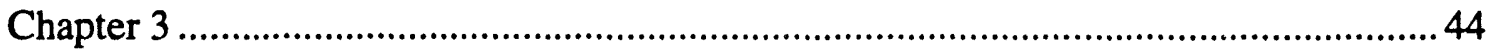

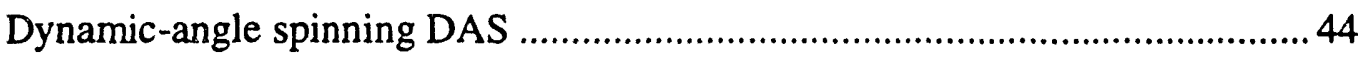

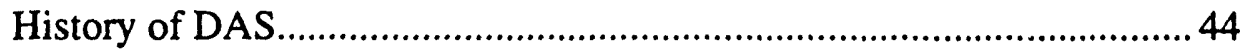

Spinning Sidebands .....................................................................5 52

Linewidths in DAS (Homonuclear Dipole-Dipole Interaction)............. 69

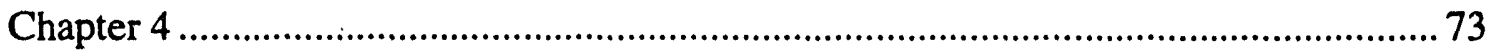

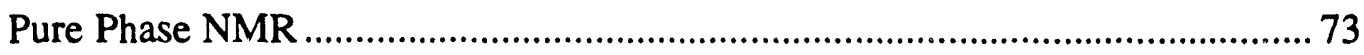

Pure-Absorption Mode Acquisition Methods ..................................... 74

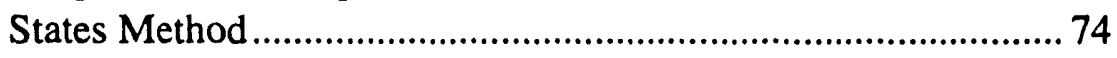

Time Proportional Phase Incrementation .................................. 76

Whole Echo Acquisition ......................................................... 77

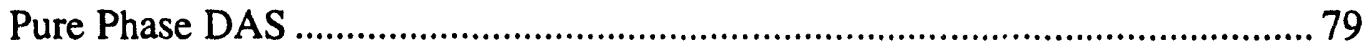

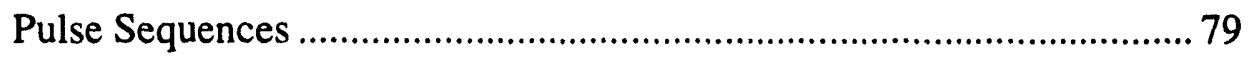

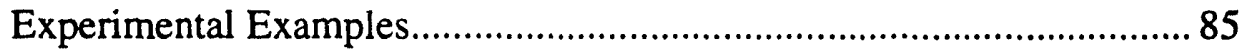

Signal-to-Noise Ratio Enhancements............................................... 88 


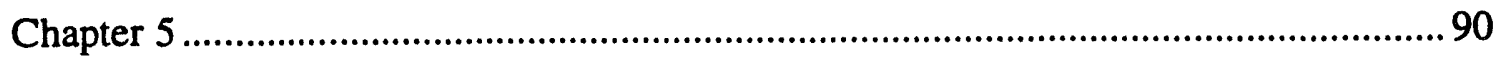

Cross Polarization ......................................................................................... 90

History ................................................................................................. 90

Spinning Effects on CP of Quadrupolar Nuclei ...................................... 91

Theory ....................................................................................92

Experiments on Sodium Pyruvate and Sodium Hydroxide......... 105

Cross Polarization Results and Discussion .................................. 107

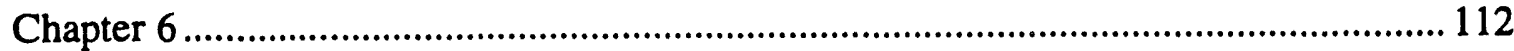

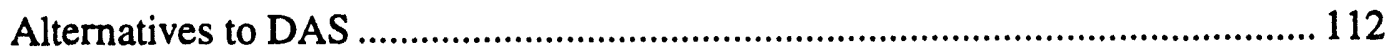

Double Rotation (DOR) ................................................................... 112

Magic-Angle Hopping (MAH)............................................................. 121

Dynamic-Angle Hopping (DAH) ......................................................... 130

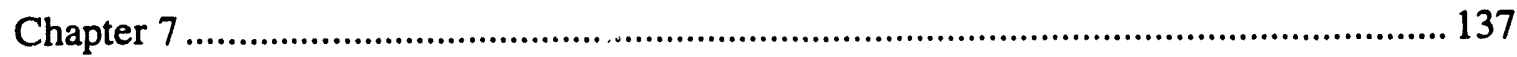

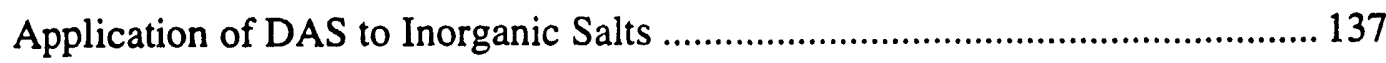

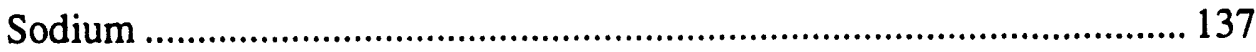

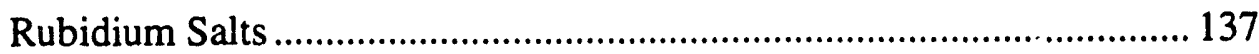

Improvements from Multiple-Field DAS...................................... 145

Improvements from MAS-Detected DAS ..................................... 147

Theory of Coupling Constants from Crystal Structure ................ 150

Application of CPDAS to organic compounds ................................................... 151

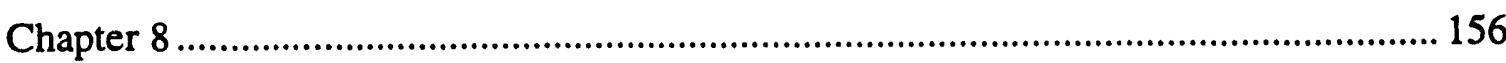

Application of DAS to silicate materials...................................................... 156

Crystalline Silicates............................................................................. 156

Amorphous Silicates ……………………............................................ 168

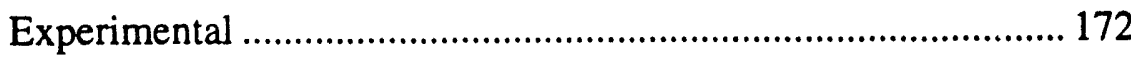

Amorphous Silica ……......................................................... 173

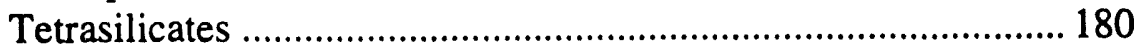

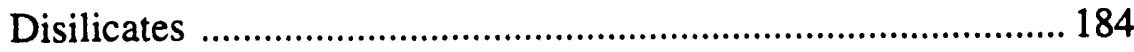

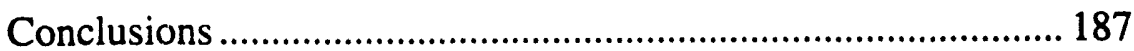

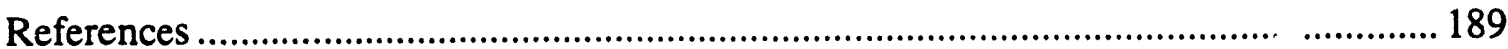

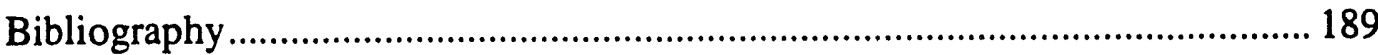

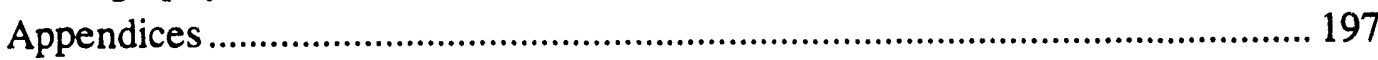

Computer programs.......................................................................... 197

CQP - VAS Spectral Simulation Program ................................. 198

MINUITCQ - VAS Least Squares Fitting Program..................... 210 


\section{List of Figures}

Figure 2.1 Euler Angle Definitions 6

Figure 2.2 90-90 Echo Coherence Pathway ................................................................ 29

Figure 2.3 Zeeman and Quadrupolar Energy Splitting for $I=3 / 2$ nucleus................... 32

Figure 2.4 PAS to ROTOR to LAB rotations..............................................................35

Figure 2.5 Static CSA (a), 1st Order (b) and 2nd Order (c) Quadrupolar

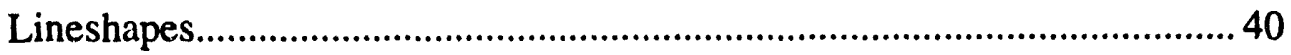

Figure 2.6 MAS CSA (a) and 2nd Order (b) Quadrupolar Lineshapes.......................... 41

Figure 2.7 Quadrupolar VAS Spectra........................................................................... 42

Figure 3.1 2nd and 4th Order Legendre Polynomials ...................................................45

Figure 3.2 DAS Experiment and Pulse Sequence ……………………………..............46

Figure 3.3 DAS Pulse Sequence Coherence Pathway ………......................................... 47

Figure 3.4 DAS Angle Pairs .............................................................................. 49

Figure 3.5 Example 1D DAS Spectrum of Sodium Oxalate ..........................................50

Figure 3.6 Example 2D DAS Spectrum of Sodium Oxalate ..........................................51

Figure 3.7 Sidebands in MAS Spectra of CSA and Second-Order Quadrupolar

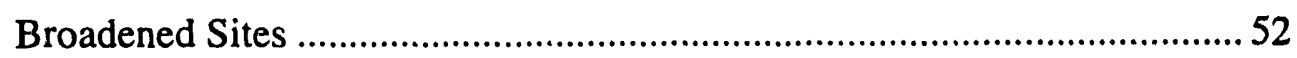

Figure 3.8 Redefined DAS Pulse Sequence for Spinning Sideband Calculation............59

Figure 3.9 Sidebands in $k=1$ DAS 2D Spectrum of $\mathrm{RbClO}_{4}$ at $11.7 \mathrm{~T}$...........................6 60

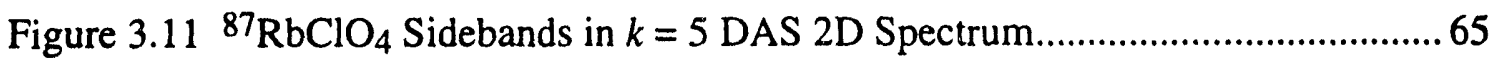

Figure $3.12{ }^{87} \mathrm{RbClO}_{4}$ Sidebands in $k=0.8$ DAS 2D Spectrum...................................66

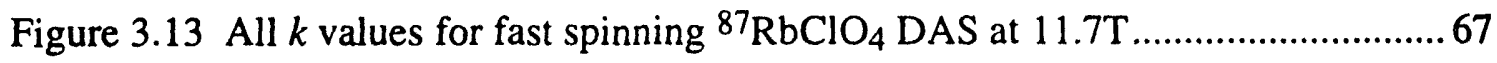

Figure 3.14 Homonuclear Dipolar Linewidth versus Spinning Angle ........................... 70

Figure 3.15 Dynamic-Angle Spinning Linewidths as a function of $k \ldots \ldots \ldots \ldots \ldots \ldots \ldots \ldots \ldots \ldots . . . . . .12$

Figure 4.1 Pure-Absorption mode and Mixed-Phase 2D NMR Spectra ......................... 74

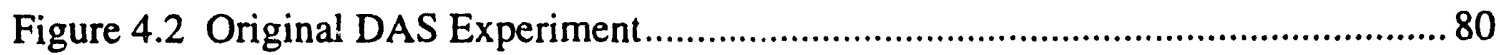

Figure 4.3 Modified DAS Experiment …………….............................................. 81

Figure 4.4 Hypercomplex DAS Experiment ................................................................ 82

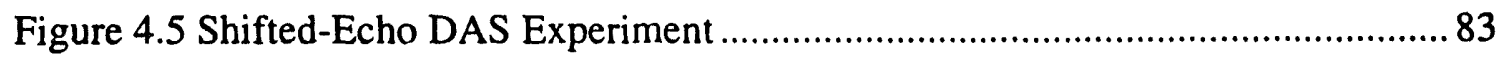

Figure 4.6 Hypercomplex Shifted-Echo DAS Experiment ........................................... 84

Figure 5.4 CP Efficiency versus Spinning Angle ........................................................ 107

Figure 5.5 Comparison of CPMAS, MAS and ZPMAS Experiments ............................ 109 
Figure 5.6 Comparison of CPDAS versus DAS ……….........................................110

Figure 6.1 DOR Rotor and Rotations ..................................................................... 112

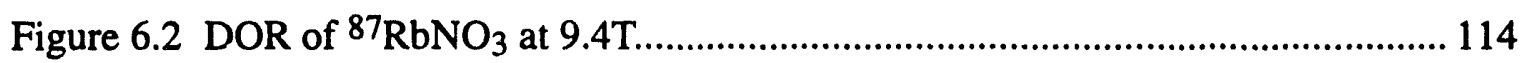

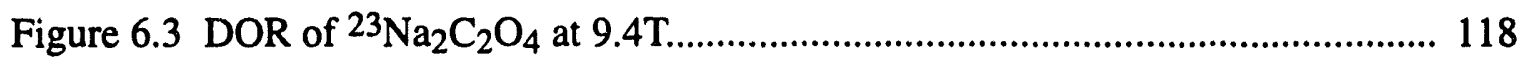

Figure 6.4 DOR of ${ }^{23} \mathrm{Na}_{2} \mathrm{C}_{2} \mathrm{O}_{4}$ at 9.4T............................................................. 121

Figure 6.5 Magic-Angle Hopping Experiment............................................................ 122

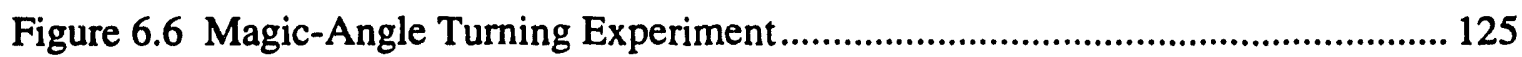

Figure 6.7 Magic-Angle Turning Experiment with $\pi$ Pulses........................................... 127

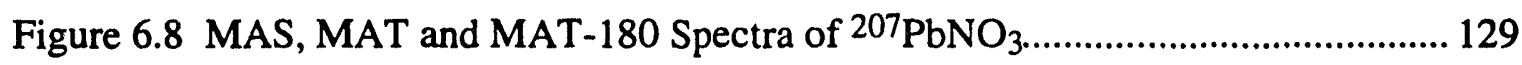

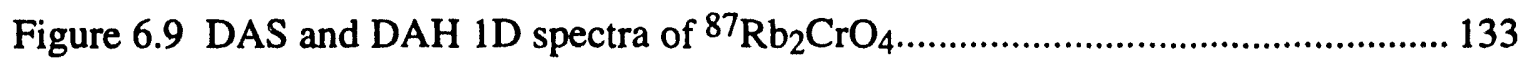

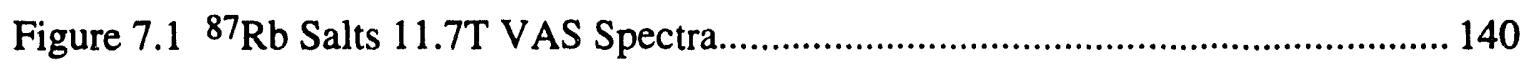

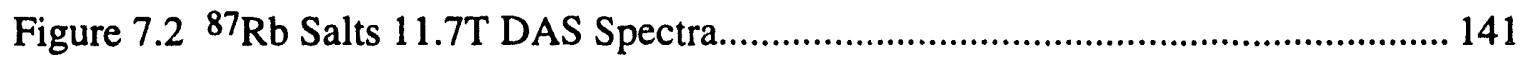

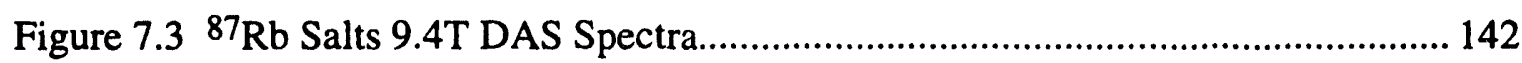

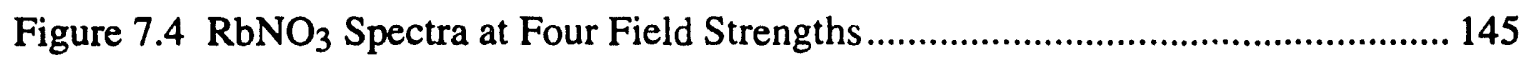

Figure 7.5 $\mathrm{RbNO}_{3}$ Linear Regression of Isotropic Shifts versus $1 / B_{0}^{2} \ldots \ldots \ldots \ldots \ldots \ldots \ldots \ldots \ldots . . .146$

Figure 7.6 $\mathrm{RbNO}_{3}$ 9.4T 2D MAS detected DAS Contour Plot....................................... 148

Figure 7.7 $\mathrm{RbNO}_{3}$ 11.7T 2D MAS detected DAS Contour Plot.................................... 148

Figure 7.8 $\mathrm{RbNO}_{3}$ Single Site MAS Slices and Simulations at 11.7T ............................ 149

Figure $7.9{ }^{1} \mathrm{H}$ Decoupled 2D ${ }^{17} \mathrm{O}$ CPDAS Spectrum of L-Alanine at 7.0T................... 153

Figure 7.10 Undecoupled 17O MAS and DAS Spectra of L-Alanine at 11.7T.............. 154

Figure 8.1 Crystal Structures of Some Pyroxene Silicate Minerals ................................ 159

Figure 8.2 Crystalline 9.4T DAS, DOR and MAS Spectra.......................................... 161

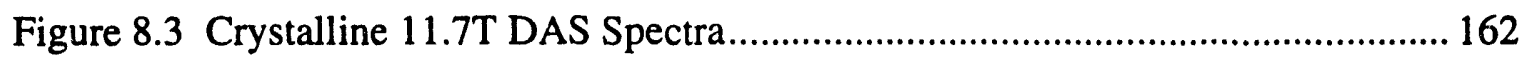

Figure 8.4 Crystalline Diopside 9.4T and 11.7T MAS Spectra ……........................... 165

Figure 8.5 Comparison of $\mathrm{A}_{2} \mathrm{O}_{3}$ Crystal and Glass Lattice Structures........................... 169

Figure 8.6 Insertion of Modifying Cations into Silicate Glasses ................................... 170

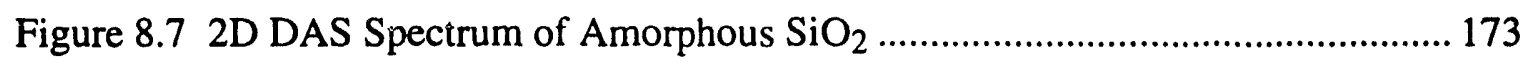

Figure 8.8 $\mathrm{SiO}_{2}$ Anisotropic Slices and Simulations.................................................. 175

Figure 8.9 Electric Field Gradient Model Compound .............................................. 176

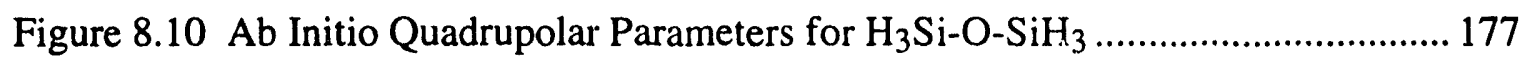

Figure 8.11 Amorphous $\mathrm{SiO}_{2}$ Bond Angle Distribution ............................................ 178

Figure 8.12 2D DAS Spectra of Tetrasilicate Glasses................................................... 181 


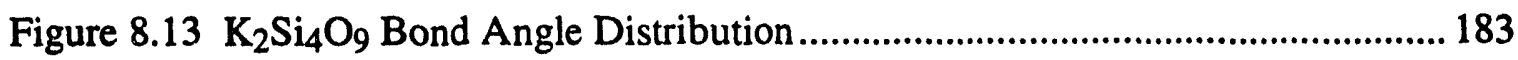

Figure 8.14 DAS Spectrum of $\mathrm{K}_{2} \mathrm{Si}_{2} \mathrm{O}_{5}$ glass at 11.7T ............................................. 185

Figure $8.15 \quad \mathrm{~K}_{2} \mathrm{Si}_{2} \mathrm{O}_{5}$ Bond Angle Distribution......................................................... 186 


\section{List of Tables}

Table 2.1 Second-rank reduced Wigner rotation matrix elements $d_{n m}^{(2)}(\beta)$................. 7

Table 2.2 Cosine Expansion Coefficients.................................................................... 34

Table 2.3 Coefficients in Anisotropic Cosine Expansion for the 2nd-Order Quadrupolar Correction Under Fast Spinning Conditions. ............................... 38

Table 4.1 Signal-to-Noise Ratio Enhancements For a Variety of Pulse Sequences...... 89

Table 5.1 Signal to Noise Ratios in CPDAS and Normal DAS ................................... 111

Table 6.1 Magic-Angle Hopping Experimental Phase Cycle..................................... 122

Table 7.1 ${ }^{87} \mathrm{Rb}$ Isotropic Shifts and Coupling Products............................................. 143

Table 7.2 Previously Measured ${ }^{87} \mathrm{Rb}$ Isotropic Chemical Shifts and Quadrupolar

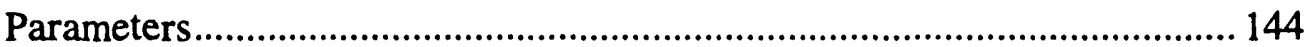

Table $7.3{ }^{87} \mathrm{RbNO}_{3}$ Multiple Field DAS Results .................................................. 147

Table $7.4{ }^{87} \mathrm{Rb}$ Isotropic Shifts from MAS Simulations ............................................. 150

Table 7.5 ${ }^{87} \mathrm{RbNO}_{3}$ EFG Values From Crystal Structure ........................................ 151

Table 8.1 Isotropic Chemical Shifts and Quadrupolar Coupling Products from

Two Field Studies ........................................................................... 164

Table 8.2 Diopside Quadrupolar Parameters............................................................. 166

Table 8.3 $\mathrm{SiO}_{2}$ Anisotropic Slice Fits..................................................................... 174

Table 8.4 $\mathrm{K}_{2} \mathrm{Si}_{4} \mathrm{O}_{9}$ Anisotropic Slice Fits from 9.4T and 11.7T DAS Spectrum......... 182

Table $8.5 \mathrm{~K}_{2} \mathrm{Si}_{2} \mathrm{O}_{5}$ Anisotropic Slice Fits........................................................... 186 


\section{List of Symbols}

Symbol

$A_{l m}^{\lambda}, a_{l m}^{\lambda}, R_{l m}^{\lambda}, r_{l m}^{\lambda}, \rho_{l m}^{\lambda}, \sigma_{l m}^{\lambda}$

$A_{N}, S_{N}, S_{N_{1}, N_{2}}$

$\left(\alpha^{\lambda}, \beta^{\lambda}, \gamma^{\lambda}\right)$

$B_{0}$

$C P$

CSA

$C_{Q}$

$\left\langle L, M \mid l, l^{\prime}, m, M-m\right\rangle$

DAS

DOR

$D_{m, n}^{(l)}\left(\alpha^{\lambda}, \beta^{\lambda}, \gamma^{\lambda}\right)$

$D$

$\delta(z)$

$e Q$

$e q$

FID

$\gamma$

$\eta_{\lambda}$

$\hbar$

$H_{\lambda}$

$\tilde{H}_{\lambda}$

$|I, m\rangle$
Description

Spatial tensor operators for the $\lambda$ interaction

Sideband amplitude functions

Euler angles used in rotations between reference frames

Static magnetic field Cross polarization

Chemical shift anisotropy

Quadrupolar coupling constant

Clebsch-Gordon Coefficient

Dynamic-angle spinning

Double rotation

Wigner rotation matrix element

Dipolar (either heteronuclear or homonuclear) interaction

Dirac delta function

Nuclear quadrupolar moment

Electric field gradient magnitude

Free induction decay

Nuclear gyromagnetic ratio

Asymmetry parameter for the $\lambda$ interaction

Planck's constant

Hamiltonian for $\lambda$ interaction

Rotating frame Hamiltonian for $\lambda$ interaction

Spin state ket for an $I$ spin in state $m$ 


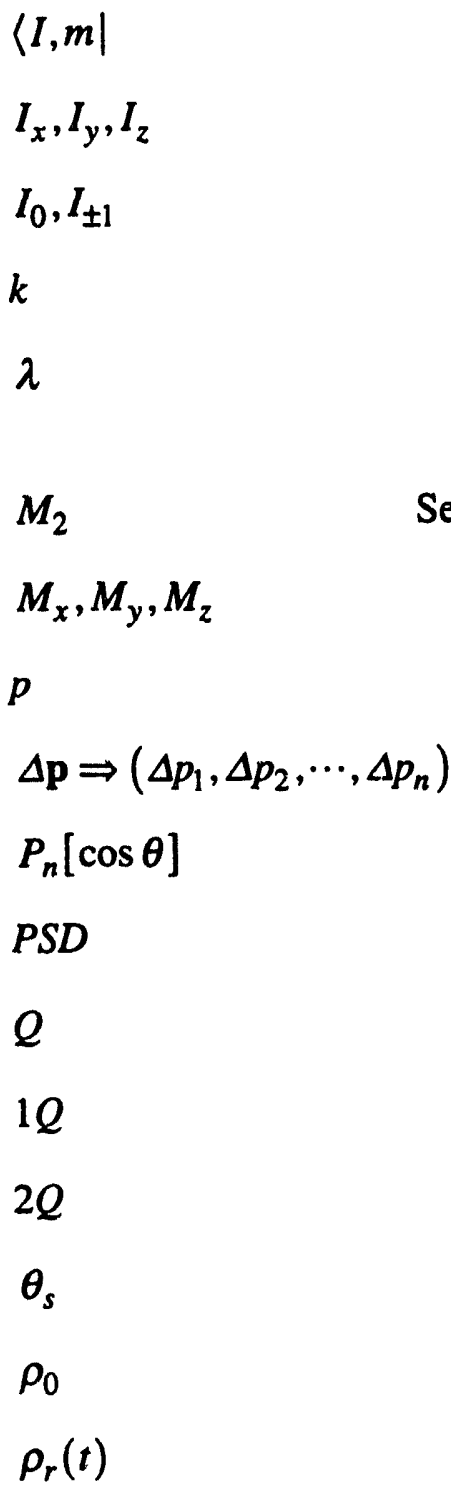

SEDAS

$S(t), S\left(t_{1}, t_{2}\right)$

$S(\omega), S\left(\omega_{1}, \omega_{2}\right)$

$T_{1}, T_{2}$

TPPI

$T_{l m}^{\lambda}$

$U(t), U^{\dagger}(t)$

V
Spin state bra for an $I$ spin in state $m$

Cartesian spin operators

Spherical normalized spin operators

DAS time ratio constant

Type of spin interaction (chemical shift anisotropy, quadrupolar: dipolar, etc.)

Second moment (from either dipolar or other interactions)

Cartesian sample magnetization components

Coherence level

Coherence transfer pathway vector

Order- $n$ Legendre polynomials of $\cos \theta$

Phase sensitive detection (or quadrature)

Quadrupolar interaction

First-order quadrupolar interaction

Second-order quadrupolar interaction

Shearing transformation shearing angle

Equilibrium density matrix describing spin system

Reduced density matrix describing spin system

Shifted echo dynamic-angle spinning

Time domain NMR signal ( 1 or 2 dimensional)

Frequency domain NMR signal (1 or 2 dimensional)

Spin-lattice and spin-spin relaxation times

Time proportional phase incrementation

Spin tenso operators for the $\lambda$ interaction

Unitary time evolution operators

Perturbing Hamiltonian in perturbation theory 
$V_{x x}, V_{y y}, V_{z z}$

$\omega_{1}$

$\omega_{l}$

$\omega_{r}$

$\omega_{\text {rot }}$

$\omega_{Q}$
Principal axes components of the electric field gradient Radiofrequency oscillating magnetic field Larmor precession frequency

Sample spinning speed

Rotating frame precession frequency

Quadrupolar frequency 


\section{Acknowledgments}

Over the last four years at Berkeley, many people have shaped me in one way or another. I cannot thank everyone, but there are a few to whom I would like to give particular thanks. I would like to thank Professor A. Pines who has given me an environment in which I could succeed and ideas to push me forward and Dr. Gerard Chingas for keeping the spectrometers working and providing expert advice on a plethora of topics. I would like to thank Professors Karl Mueller (Penn State), Joe Zwanziger (Indiana University), Phil Grandinetti (Ohio State), Brad Chmelka (UC Santa Barbara) and Dr. Steve Rucker (Exxon) who helped a young graduate student formulate useful ideas and pointed me in the right direction. I would like to thank Brian F. Volkman, Welcome Rex Anthony Huff, Indiana (a particularly stupid black Labrador retriever) and Toby (a second equally dense German shepherd) who were lighthearted roommates who helped to keep me sane, even when my house was shaking in an earthquake (October 1989) or burning down (October 1991). I would thank Dave Williams, perhaps the greatest landlord known to mankind (also a fellow fire victim). I especially thank the cities of Berkeley (Mayor Loni Hancock and the BCA in particular) and Oakland for giving me the inspiration (the parking tickets, rampant crime and homelessness, rent control, and unique fire fighting philosophies) to finish my dissertation as quickly as possible. I also thank the entire Magnetic Fielders team (formerly the Spinors) for many innings of relaxing and enjoyable chemistry league softball. I give my heartfelt thanks to Drs. Ulli Werner, Margaret Eastman, Tsong H. Chang, Yu Wue, Lyndon Emsley, Wrenn Wooten and Lucio Frydman, the knowledgeable post-doctoral fellows who passed through the lab in my years at Berkeley. Dr. Wooten and Dr. Werner in particular played important roles in setting up many of these experiments. As well, I acknowledge Mark Rosen, Sheryl Gann, Young Lee, Geoff Barrall, Joe Sachleben, Henry Long, Holly Gaede and Bruce Black, with whom I endured the life known as graduate school. Sheryl Gann 
deserves special acknowledgment for doing many of the jobs that I hated to do in getting much of this thesis work finished. I thank the National Science Foundation for providing a graduate fellowship. This research was supported by the Director, Office of Energy Research, Office of Basic Energy Sciences, Materials Science Division of the U.S. Department of Energy under Contract No. DE-AC03-76SF00098 and is gratefully acknowledged. Finally, I must thank Dione Carmichael, the group administrative assistant and mother to us all, without whom I am sure none of this would have been accomplished. 


\section{Chapter 1}

\section{Introduction}

Nuclear magnetic resonance (NMR) spectroscopy is one of the most important techniques used in chemistry today. The use of liquid state ${ }^{1} \mathrm{H}$ spectra to identify organic compounds, along with infra-red spectroscopy, mass spectrometry and x-ray diffraction, has essentially replaced the chemical techniques used in previous decades. In addition, the use of NMR to examine large biologically active peptides, proteins, nucleic acids and sugars has greatly enhanced the speed and accuracy of structure determination, as well as giving site specific dynamical information. Finally, the use of magnetic res/nance to do medical imaging has significantly improved the diagnostic capability of the medical community. Magnetic resonance imaging has provided a tool to do non-invasive examination of patients without the use of harmful high-energy radiation (i.e. x-rays) or radioactive chemical isotopes (i.e. positron emission tomography). However, all of these techniques primarily deal with molecules in solution. The application of magnetic resonance to solid state samples has been limited mostly to chemical physics and physical chemistry laboratories at large research institutes. The last ten years have seen an explosion in the use of solid state NMR. Primarily this has come about due to advances both in multiple-pulse techniques and in sample reorientation experiments. The single most important solid state NMR technique to be developed is cross-polarized magic-angle spinning (CPMAS). This experiment allows routine rapid collection of proton decoupled carbon-13 spectra with nearly liquid-like resolution. The last five years have seen the further advances of multi-dimensional solid state experiments which have continued to enhance information content in solid state NMR spectra.

Throughout this thesis, I will discuss experiments I carried out in the laboratory of Prof. A. Pines at the University of California, Berkeley. In particular, I will describe the advances we made with the technique of dynamic-angle spinning NMR. This technique 
was designed to obtain high-resolution liquid-like spectra of solid samples containing quadrupolar nuclei. In this work, I will show the application of this technique primarily to ${ }^{87} \mathrm{Rb}$ and ${ }^{17} \mathrm{O}$ containing compounds. This is by no means a complete or systematic study of all such compounds, but merely a demonstration of the possibilities for the ultimate use of dynamic-angle spinning experiments once these experiments become as routine as CPMAS. 


\section{Chapter 2}

\section{Theory of Nuclear Magnetic Resonance}

To understand the techniques of nuclear magnetic resonance spectroscopy which I will discuss throughout this thesis, I will first need to introduce some of the fundamentals of quantum mechanics which will be used to describe the application of static and rotating frame magnetic fields to a system composed of a large number of spins. There are a large number of good fundamental and more advanced texts on quantum mechanics. ${ }^{1-5}$ Also I would direct the reader to additional books written specifically about nuclear magnetic resonance which provide the basis for much of the theory presented in this chapter. ${ }^{6-}$ 13 Finally the doctoral theses of Sun, Mueller and Chmelka all provide additional detailed information about the techniques presented here. ${ }^{14-16}$ Before doing a complete description of NMR in terms of quantum mechanics, I will first introduce the classical magnetization vector picture.

\section{Classical Magnetization Description}

The magnetization picture assumes that an ensemble of spins may be treated as an overall macroscopic magnetization which rotates about the axis of the applied magnetic field at a characteristic Larmor frequency. This description may adequately characterize some simple single- and multiple-pulse experiments. The Larmor energy splitting is given by the formula

$$
\Delta E_{l}=\hbar \gamma B_{0}=\hbar \omega_{l}
$$

where $\hbar$ is Planck's constant, $\gamma$ is the gyromagnetic ratio and $B_{0}$ is the static magnetic field. A useful concept in describing NMR experiments is the rotating frame of the Larmor frequency. Mathematically we transform from a fixed laboratory frame to a frame which is rotating at the Larmor frequency about the zlab axis defined by the static mag- 
netic field. In this frame the Cartesian coordinates $\left(x_{l a b}, y_{l a b}, z_{l a b}\right)$ will be transformed according to the following relationships:

$$
\begin{aligned}
& x_{\text {rot }}=x_{l a b} \cos \omega_{l} t+y_{l a b} \sin \omega_{l} t \\
& y_{\text {rot }}=y_{l a b} \cos \omega_{l} t-x_{l a b} \sin \omega_{l} t \\
& z_{\text {rot }}=z_{l a b}
\end{aligned}
$$

where $\left(x_{r o t}, y_{\text {rot }}, z_{r o l}\right)$ are the rotating frame coordinates and $\omega_{l}$ is the Larmor frequency. First, in this frame the effective $z_{r o t}$ axis magnetic field is exactly canceled for on resonance spins and appears as a much smaller field $B_{\text {offset }}=B_{0}-\left(\frac{\omega_{1}-\delta}{\gamma}\right)$ to spins off resonance by $\delta$. Second, in the rotating frame a magnetic field oscillating about the laboratory $x$-axis (i.e. the applied radio frequency pulse) will appear as the sum of two oscillating magnetic fields. One oscillates at a frequency $\omega_{\text {effective }}=\omega_{\text {applied }}-\omega_{l}$ and the other at a frequency $\omega_{\text {effective }}=-\omega_{\text {applied }}-\omega_{l}$. Only the former, low frequency, rotating frame magnetic field can affect the net magnetization of the sample. In the presence of a strong radio frequency $(\mathrm{RF})$ pulse, the net $\mathrm{z}$ magnetization will begin to process about the effective magnetic field which is the vector sum of the $B_{\text {offset }}$ along the $z_{\text {rot }}$ axis and $B_{1}$ along the $x_{\text {rot }}$ axis. For on-resonance spins, a $\pi / 2$ pulse consists of RF field applied for a time such that $\frac{\pi}{2}=\gamma B_{\text {effective }}$. For spins which are only slightly off resonance $\left(B_{1}>B_{\text {offset }}\right)$, this RF pulse will effectively be a "90 pulse" to a good approximation and the $z_{r o t}$ magnetization will rotate about the $x_{r o t}$ axes to generate a large $y_{r o t}$ magnetization. When the strong RF pulse is turned off, the effective magnetic field returns to the purely $B_{o f f s e t}$ state along the $z_{r o t}$ axis. At this point, the net $y_{r o t}$ magnetization will begin to process about the $z_{\text {rot }}$ axis. This rotating magnetization will appear as an oscillating magnetic field in the laboratory frame and can be detected with the same coil used to generate the RF pulse. The detected signal called a free induction decay (FID) contains the Fourier sum of all the frequencies present in the sample. The rotating frame Bloch equations describe this magnetization evolution in classical terms and are shown below. 


$$
\begin{aligned}
& \frac{d M_{z}}{d t}=-\gamma M_{y} B_{1}+\frac{M_{0}-M_{z}}{T_{1}} \\
& \frac{d M_{x}}{d t}=\gamma M_{y} B_{o f f s e t}-\frac{M_{x}}{T_{2}} \\
& \frac{d M_{y}}{d t}=\gamma\left(M_{z} B_{1}-M_{x} B_{o f f s e t}\right)-\frac{M_{y}}{T_{2}}
\end{aligned}
$$

These equations show the excitation and free induction decay behavior described. In addition, they include relaxation terms not yet discussed. The $T_{1}$ (longitudinal) and $T_{2}$ (transverse) relaxation rates are described more completely in some of the basic NMR texts. For the purpose of this thesis, they will always be treated classically, meaning, an irreversible exponential loss of coherence of both transverse $\left(M_{x}\right.$ and $\left.M_{y}\right)$ and longitudinal $\left(M_{z}\right)$ magnetization components. In quantum mechanical terms, they will lead to an overall exponential damping of density matrix elements (such as $I_{x}, I_{y}$ or $I_{z}$ ) to equilib$\operatorname{rium}\left(I_{z}\right)$.

\section{Quantum Mechanical Description}

Any discussion of NMR will ultimately be limited if only the Bloch equations are used to describe the system. To this end, the quantum mechanical fundamentals will be discussed in the next section.

\section{Rotations and Tensors}

One of the most fundamental concepts in NMR is that of rotations. Many rather complex problems in NMR spectroscopy (and other physical sciences) are greatly simplified by judicious choice of reference frame. The use of Wigner rotation matrices to simplify the mathematics of rotations has been well described in books by Rose ${ }^{17}$, Edmonds ${ }^{18}$ and Zare ${ }^{19}$. Figure 2.1 shows the definition of the three Euler angles $(\alpha, \beta, \gamma)$ as used in Wigner rotation matrices $D_{m, n}^{(l)}(\alpha, \beta, \gamma)$. One use of Wigner rotation matrices is to rotate an object with tensor properties between frames of reference. 
Suppose we have a tensor $A$ of rank $l$ with elements $A_{l m}$ which we wish to rotate into a
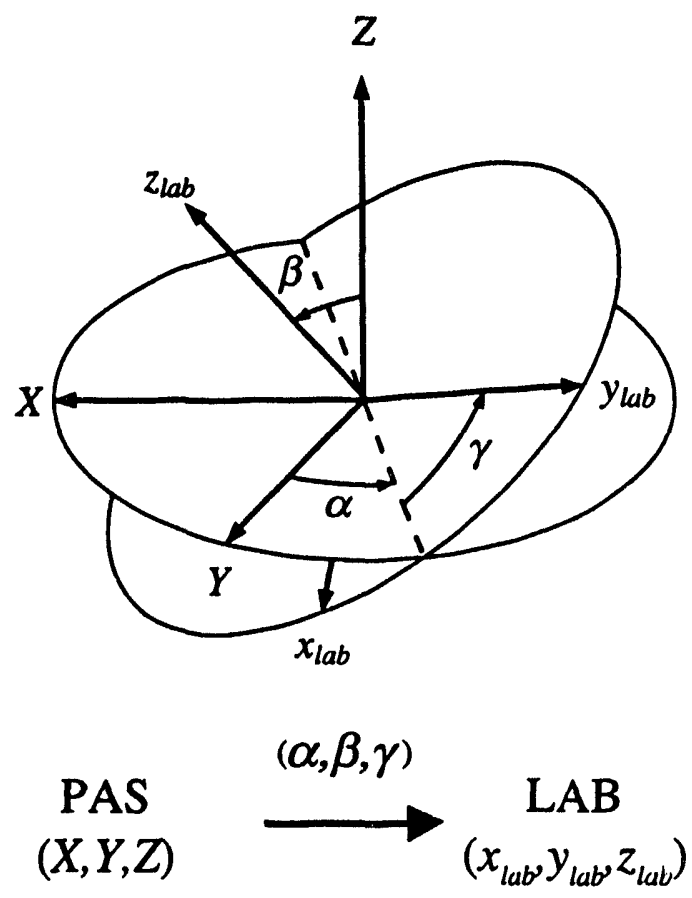

Figure 2.1 Euler Angle Definitions. The first rotation is of size $\alpha$ about the $Z$ axis, followed by a rotation of size $\beta$ about the rotated $\gamma$ axis and finally a rotation of size $\gamma$ about the rotated $Z$ axis to arrive at the new $x_{l a b}, y_{l a b}$ and $z_{l a b}$ axes.

new frame. In this new frame, the resulting tensor $R_{l}$ will have elements $R_{l m}$ given by the following expression.

$$
R_{l m}=\sum_{n=-l}^{l} D_{n m}^{(l)}(\alpha, \beta, \gamma) A_{l n}
$$

This expression may be numerically simplified by expressing $D_{n m}^{(l)}(\alpha, \beta, \gamma)$ in terms of exponentials and reduced Wigner rotation matrix elements, $d_{n m}^{(l)}(\beta)$.

$$
D_{m n}^{(l)}(\alpha, \beta, \gamma)=e^{-i(m \alpha+n \gamma)} d_{m n}^{(l)}(\beta)
$$

The expressions for each of the reduced Wigner rotation matrices can be found in any of the previously mentioned sources. For most of the work in this thesis, I will deal primarily with second rank tensors in both spin and spatial coordinates. Therefore, for refer- 
ence, I include a table (2.1) of second-rank reduced Wigner matrices, but this is by no means a complete set of matrices for all possible applications.

\begin{tabular}{|c|c|c|c|c|c|}
\hline$n$ & 2 & $m$ & & \\
\hline \hline 2 & $\left(\frac{1+\cos \beta}{2}\right)^{2}$ & $-\frac{1+\cos \beta}{2} \sin \beta$ & $\sqrt{\frac{3}{8}} \sin ^{2} \beta$ & $-\frac{1-\cos \beta}{2} \sin \beta$ & $\left(\frac{1-\cos \beta}{2}\right)^{2}$ \\
\hline 1 & $\frac{1+\cos \beta}{2} \sin \beta$ & $\cos ^{2} \beta-\frac{1-\cos \beta}{2}$ & $-\sqrt{\frac{3}{8}} \sin 2 \beta$ & $\frac{1+\cos \beta}{2}-\cos ^{2} \beta$ & $-\frac{1-\cos \beta}{2} \sin \beta$ \\
\hline 0 & $\sqrt{\frac{3}{8}} \sin ^{2} \beta$ & $\sqrt{\frac{3}{8}} \sin 2 \beta$ & $\frac{3 \cos ^{2} \beta-1}{2}$ & $-\sqrt{\frac{3}{8}} \sin 2 \beta$ & $\sqrt{\frac{3}{8}} \sin ^{2} \beta$ \\
\hline-1 & $\frac{1-\cos \beta}{2} \sin \beta$ & $\frac{1+\cos \beta}{2}-\cos { }^{2} \beta$ & $\sqrt{\frac{3}{8}} \sin 2 \beta$ & $\cos { }^{2} \beta-\frac{1-\cos \beta}{2}$ & $-\frac{1+\cos \beta}{2} \sin \beta$ \\
\hline-2 & $\left(\frac{1-\cos \beta}{2}\right)^{2}$ & $\frac{1-\cos \beta}{2} \sin \beta$ & $\sqrt{\frac{3}{8}} \sin ^{2} \beta$ & $\frac{1+\cos \beta}{2} \sin \beta$ & $\left(\frac{1+\cos \beta}{2}\right)^{2}$ \\
\hline
\end{tabular}

Table 2.1 Second-rank reduced Wigner rotation matrix elements $d_{n m}^{(2)}(\beta)$. These elements may be used in conjunction with equation 2.4 and 2.5 to facilitate rotation of tensors.

\section{Perturbation Theory}

To analyze the effect of adding a small internal Hamiltonian, like chemical shift, to the overall system described by a large external Hamiltonian, I will use standard static perturbation theory. Perturbation theory has been described previously in a large number of locations; any good quantum mechanics text will include a section on this topic. Briefly, I will sketch the basics and their application to NMR. Given an arbitrary Hamiltonian which is the sum of two parts, one large (for example the Zeeman interaction) and the other small (the chemical shift or quadrupolar interactions), a starting point is to assume that the eigenvalues and eigenstates are those of the large Hamiltonian only and then add correction terms, as in a Taylor series expansion. Shown below is the derivation of the correction to both the eigenvalues and eigenstates for a general Hamiltonian (equation 2.6) which is very similar to that found in Baym.'

$$
H=H_{0}+V
$$


where the eigenstates $|n\rangle$ and eigenvalues $\varepsilon_{n}$ of $H_{0}$, the dominant interaction, are known exactly

$$
H_{0}|n\rangle=\varepsilon_{n}|n\rangle
$$

It is assumed that the actual eigenvalues and eigenstates will be very similar to those of the large $H_{0}$ Hamiltonian. This assumption will be analytically true as the size of the perturbation $V$ goes to zero. Therefore, we will arbitrarily redefine our Hamiltonian with a parameter which may be varied between 0 and 1 (ultimately we will let $\lambda$ go to 1 ).

$$
H=H_{0}+\lambda V
$$

Now we can confidently expand the actual eigenvalues $E_{N}$ and eigenstates $|N\rangle$ below.

$$
\begin{aligned}
& |N\rangle=|n\rangle+\lambda\left|N^{(1)}\right\rangle+\lambda^{2}\left|N^{(2)}\right\rangle+\lambda^{3}\left|N^{(3)}\right\rangle+\cdots \\
& E_{N}=\varepsilon_{n}+\lambda E_{N}^{(1)}+\lambda^{2} E_{N}^{(2)}+\lambda^{3} E_{N}^{(3)}+\cdots
\end{aligned}
$$

Now we assume that the eigenstates of $H_{0}$ are normalized to $1(\langle n \mid n\rangle=1)$ and we will choose the normalization of $|N\rangle$ so that $\langle n \mid N\rangle=1$ also.

$$
\langle n \mid N\rangle=1=\langle n \mid n\rangle+\lambda\left\langle n \mid N^{(1)}\right\rangle+\lambda^{2}\left\langle n \mid N^{(2)}\right\rangle+\lambda^{3}\left\langle n \mid N^{(3)}\right\rangle+\cdots
$$

This implies that for an arbitrary $\lambda$ each correction term $\left|N^{(i)}\right\rangle$ is orthoganal to the original basis state $|n\rangle$.

$$
\left\langle n \mid N^{(i)}\right\rangle=0 \quad \text { for } \quad i=1,2,3, \ldots
$$

Now we can write the Schödinger equation for the complete Hamiltonian.

$$
H|N\rangle=\left(H_{0}+\lambda V\right)|N\rangle=E_{N}|N\rangle
$$

Collecting terms on both sides of the equation with the same power of $\lambda$ yields the following set of equations. 


$$
\begin{aligned}
& \lambda^{0} \rightarrow H_{0}|n\rangle=\varepsilon_{n}|n\rangle \\
& \lambda^{1} \rightarrow H_{0}\left|N^{(1)}\right\rangle+V|n\rangle=E_{N}^{(1)}|n\rangle+\varepsilon_{n}\left|N^{(1)}\right\rangle \\
& \lambda^{2} \rightarrow H_{0}\left|N^{(2)}\right\rangle+V\left|N^{(1)}\right\rangle=E_{N}^{(2)}|n\rangle+E_{N}^{(1)}\left|N^{(1)}\right\rangle+\varepsilon_{n}\left|N^{(2)}\right\rangle \\
& \quad \vdots \\
& \lambda^{k} \rightarrow H_{0}\left|N^{(k)}\right\rangle+V\left|N^{(k-1)}\right\rangle=E_{N}^{(k)}|n\rangle+E_{N}^{(k-1)}\left|N^{(1)}\right\rangle+\cdots+\varepsilon_{n}\left|N^{(k)}\right\rangle
\end{aligned}
$$

To determine the first order eigenvalue correction $E_{N}^{(1)}$, we need to take the scalar product of the second equation with $\langle n|$. By our previously defined orthogonality and normalization equations, this produces the eigenvalue correction term.

$$
\begin{aligned}
\left\langle n\left|H_{0}\right| N^{(1)}\right\rangle+\langle n|V| n\rangle & =\left\langle n\left|E_{N}^{(1)}\right| n\right\rangle+\left\langle n\left|\varepsilon_{n}\right| N^{(1)}\right\rangle \\
\varepsilon_{n}\left\langle n \mid N^{(1)}\right\rangle+\langle n|V| n\rangle & =E_{N}^{(1)}\langle n \mid n\rangle+\varepsilon_{n}\left\langle n \mid N^{(1)}\right\rangle \\
\langle n|V| n\rangle & =E_{N}^{(1)}
\end{aligned}
$$

This result states that to first-order in perturbation theory, we need merely to calculate the eigenvalues using the original basis set. This is equivalent to simply ignoring the non-diagonal matrix elements of $V$. For most NMR calculations we perform, this rather crude approximation is sufficient. In some cases where $V$ is large, however, higher order terms may be needed (for example the second-order quadrupolar shift). To calculate the higher order eigenvalue corrections, we take the scalar product of the $\lambda^{k}$ equation with $\langle n|$. This produces the following result.

$$
E_{N}^{(k)}=\left\langle n|V| N^{(k-1)}\right\rangle
$$

This expression shows that once we know the $(k-1)$ th correction to the eigenstate, we can calculate the $k$ th correction to the eigenvalue. The first step in calculating the correction to the eigenstates is to expand the $k$ th correction eigenstate in terms of the complete basis set of $H_{0}$. Note that the sum excludes the $m=n$ state but includes all other possible eigenstates of $H_{0}$, as this state is defined as orthogonal to the correction eigenstate.

$$
\left|N^{(k)}\right\rangle=\sum_{m \neq n}|m\rangle\left\langle m \mid N^{(k)}\right\rangle
$$


Now to calculate the second order correction (higher-orders are calculated in a similar fashion) we take the scalar product of the $\lambda^{1}$ equation with a basis state $\langle m|$ (but not $\langle n|$ ).

$$
\begin{aligned}
\left\langle m\left|H_{0}\right| N^{(1)}\right\rangle+\langle m|V| n\rangle & =\left\langle m\left|E_{N}^{(1)}\right| n\right\rangle+\left\langle m\left|\varepsilon_{n}\right| N^{(1)}\right\rangle \\
\varepsilon_{m}\left\langle m \mid N^{(1)}\right\rangle+\langle m|V| n\rangle & =E_{N}^{(1)}\langle m \mid n\rangle+\varepsilon_{n}\left\langle m \mid N^{(1)}\right\rangle \\
\langle m|V| n\rangle & =\left(\varepsilon_{n}-\varepsilon_{m}\right)\left\langle m \mid N^{(1)}\right\rangle \\
\frac{\langle m|V| n\rangle}{\left(\varepsilon_{n}-\varepsilon_{m}\right)} & =\left\langle m \mid N^{(1)}\right\rangle
\end{aligned}
$$

Finally, we insert this result into equation 2.16 and take the scalar product with $\langle n| V$.

$$
E_{N}^{(2)}=\left\langle n|V| N^{(1)}\right\rangle=\sum_{m \neq n} \frac{\langle n|V| m\rangle\langle m|V| n\rangle}{\varepsilon_{n}-\varepsilon_{m}}
$$

At this point, a word of warning in general, accidental degeneracy in the $\varepsilon_{m}$ eigenvalues can lead to problems and special precautions must be taken under those conditions (this actually happens when this type of theory is applied to nuclear quadrupole resonance (NQR) experiments ${ }^{13}$ ). However, for most NMR problems, this will not present any difficulties. An alternative way of writing our result is that the original Hamiltonian $\left(H_{0}+V\right)$ may be approximated by a diagonal Hamiltonian, in which the higher order energy correction terms appear on the diagonal.

$$
H=\sum_{n}\left(\varepsilon_{n}+E_{N}^{(1)}+E_{N}^{(2)}+\cdots\right)|n\rangle\langle n|
$$

In summary, the results of perturbation theory show that to first-order it is correct to truncate Hamiltonians, such as the chemical shift Hamiltonian in the presence of the Zeeman interaction, by ignoring all terms which do not commute with the basis (Zeeman) Hamiltonian (this is often refered to as dropping the non-secular terms). In cases where the first-order correction is very large, second-order (and possibly higher) corrections must be considered, but these must be considered using the entire perturbing Hamiltonian (as the truncated first-order Hamiltonian would actually give a zero result for any higher order correction, as it is entirely diagonal in the unperturbed basis set). 


\section{Zeeman Interaction}

In nuclear magnetic resonance, the single most important Hamiltonian is the nuclear Zeeman Hamiltonian. This is expressed in the laboratory frame below in equation 2.20 .

$$
H_{Z}=-\hbar \gamma B_{0} I_{0}=-\hbar \omega_{l} I_{0}
$$

The magnetic field, $B_{0}$, defines the $z$ lab axis, $\hbar$ is Planck's constant, $\gamma$ is the gyromagnetic ratio of the nucleus of interest and $\omega_{l}$ is the Larmor frequency. The spin operator, $I_{0}$, is defined below, as well as the two other spherical operators which make up the complete Zeeman spin operator set.

$$
\begin{aligned}
& I_{0}=I_{z} \\
& I_{ \pm 1}= \pm \frac{1}{\sqrt{2}}\left(I_{x} \pm i I_{y}\right)
\end{aligned}
$$

The most fundamental aspect of the Zeeman interaction in high magnetic field is that it is always (for the purposes of this dissertation) the largest nuclear spin interaction present in a system. This effectively means that the eigenvalues and eigenstates of the Zeeman Hamiltonian will act as a basis set for the perturbative expansion of the other important interactions. The $m$ spin states (the Zeeman eigenstates) for an isolated $I$ spin nucleus are $|I, m\rangle$. The matrix elements in this basis set for the various operators are given below.

$$
\begin{aligned}
& I_{0}|I, m\rangle=m|I, m\rangle \\
& I_{ \pm 1}|I, m\rangle=\frac{ \pm 1}{\sqrt{2}} \sqrt{(I \mp m)(I \pm m+1)}|I, m \pm 1\rangle
\end{aligned}
$$

In this description any state with $|m|>I$ is equivalent to the null state. For many of our applications, however, we will also use a density matrix formulation of quantum mechanics. In this formalism, operators can be represented as matrices which operate on a density matrix which describes the system. Matrices which represent operators are defined below. 


$$
\begin{aligned}
I_{k} & =\sum_{m, n=-I}^{I}|I, m\rangle\left\langle I, m\left|I_{k}\right| I, n\right\rangle\langle I, n| \\
& =\sum_{m, n=-I}^{I} c_{m, n}|I, m\rangle\langle I, n| \\
c_{m, n} & =\left\langle I, m\left|I_{k}\right| I, n\right\rangle
\end{aligned}
$$

A brief description of the density matrix formulation of quantum mechanics is merited here. Suppose we have a linear superposition state $\psi(t)$ (in Hilbert space) of the Zeeman Hamiltonian given below with complex coefficients $a_{m}(t)$.

$$
\psi(t)=\sum_{m=-I}^{I} a_{m}(t)|I, m\rangle
$$

This state will evolve under a time-independent Hamiltonian according to the Schrödinger equation.

$$
\begin{gathered}
i \hbar \frac{d \psi(t)}{d t}=H \psi(t) \\
\Downarrow \\
\psi(t)=e^{-i H t / \hbar} \psi(0)
\end{gathered}
$$

Alternatively, the same information may be presented in a density matrix formulation (Liouville space), where the density matrix which defines the system is given in equation 2.26 .

$$
\begin{aligned}
\rho(t) & =\psi(t) \psi^{\dagger}(t)=\sum_{m, n=-I}^{I} a_{m}(t) a_{n}^{\dagger}(t)|I, m\rangle\langle I, n| \\
& =e^{-i H t / \hbar} \psi(0) \psi^{\dagger}(0) e^{i H t / \hbar}=e^{-i H t / \hbar} \rho(0) e^{i H t / \hbar}
\end{aligned}
$$

It is in this form that most NMR experiments will be described throughout this and other works.

The initial density matrix at thermal equilibrium in a high magnetic field can be shown to be

$$
\begin{aligned}
& \rho_{0}=\frac{1}{Z} \exp \left\{-H_{Z} / k T\right\} \approx \frac{1}{(2 I+1)}\left\{1+H_{Z} / k T\right\} \\
& Z=\operatorname{tr}\left\{\exp \left\{-H_{Z} / k T\right\}\right\} \approx(2 I+1)
\end{aligned}
$$


where $k$ is Boltzmann's constant, 1 is a $(2 I+1 \times 2 I+1)$ unit matrix, and $T$ is the spin temperature. It can be shown that only the second term is observable in an NMR experiment and therefore the unit matrix may be dropped from the expression leaving the reduced density matrix $\rho_{r}$ that will be used throughout this work. (It may be noted that this represents a very small net population difference of only about $10^{-4}$.)

$$
\rho_{0} \approx \frac{\hbar \gamma B_{0}}{(2 I+1) k T} I_{0} \rightarrow \rho_{r}=I_{0}
$$

The evolution of a density matrix under a time-dependent Hamiltonian will be governed by the Liouville-von Neumann equation, which may easily be derived from the Schrödinger equation 2.25 .

$$
\frac{d \rho_{r}(t)}{d t}=-\frac{i}{\hbar}\left[H(t), \rho_{r}(t)\right]
$$

This may be solved analytically, where $T$ is the Dyson time-ordering operator. ${ }^{20,21}$

$$
\begin{aligned}
\rho_{r}(t) & =U(t) \rho_{0} U^{\dagger}(t) \\
U(t) & \left.=T \operatorname{ex} \Gamma_{l}^{\top}-\frac{i}{\hbar} \int_{0}^{t} H(s) d s\right\}
\end{aligned}
$$

Through out the remainder of this work, the subscript $r$ will be dropped from the reduced density matrix and the reader should assume that all density matrices are in the reduced form.

The final element needed to look at NMR problems is the transformation to a frame which is rotating at the frequency $-\omega_{r o t}$, as in the classical case. This may be ac complished by the following transformations. First, the rotating frame wavefunction may be related to the laboratory frame wavefunction below and may be inserted into the Schrödinger equation.

$$
\begin{aligned}
\psi(t) & =e^{i \omega_{r o t} I_{0} t} \psi_{\text {rot }}(t) \\
i \hbar \frac{d \psi(t)}{d t} & =H_{Z} \psi(t) \\
i \hbar \frac{d\left\{e^{i \omega_{\text {rot }} I_{0} t} \psi_{\text {rot }}(t)\right\}}{d t} & =-\hbar \omega_{l} I_{0} e^{i \omega_{\text {rot }} I_{0} t} \psi_{\text {rot }}(t)
\end{aligned}
$$


Expanding the various derivatives and simplifying yields the effective Hamiltonian, $\tilde{H}_{Z}$, below.

$$
\begin{aligned}
i \hbar e^{i \omega_{\text {rot }} I_{0} t} \frac{d \psi_{\text {rot }}(t)}{d t}-\hbar \omega_{r o t} I_{0} e^{i \omega_{r o t} I_{0} t} \psi_{r o t}(t) & =-\hbar \omega_{l} I_{0} e^{i \omega_{r o t} l_{0} t} \psi_{\text {rot }}(t) \\
i \hbar \frac{d \psi_{\text {rot }}(t)}{d t} & =-\hbar\left(\omega_{l}-\omega_{r o t}\right) I_{0} \psi_{\text {rot }}(t) \\
\tilde{H}_{Z} & =-\hbar\left(\omega_{l}-\omega_{r o t}\right) I_{0} \\
\rho_{\text {rot }}(t) & =e^{i \omega_{r o t} I_{0} t} \rho(t) e^{-i \omega_{r o t} I_{0} t}
\end{aligned}
$$

Since the Zeeman Hamiltonian commutes with $I_{0}$, we have merely an offset Hamiltonian in the rotating frame, just as in the classical case. The concept of transforming Hamiltonians into an interaction frame will prove essential to simplify calculations later. In this rotating frame, the Zeeman energy splitting has been effectively removed, however it will show up as an energy offset on all rotating frame measurements or calculations, since these must always be performed in the stationary laboratory frame. In practice, spectra are usually collected over a narrow bandwidth centered at the rotating frame energy and therefore the actual Zeeman splitting energy doesn't appear in most spectra.

\section{Radio frequency irradiation}

The application of radio frequency pulses to a spin system was discussed earlier in the classical description. The quantum mechanical description is very similar in all respects. We express the oscillating magnetic field created at the $\omega_{r o t}$ frequency with intensity $B_{1}$ in the Hamiltonian, $H_{R F}$. Here, the RF is applied to the system through a coil which defines the $x_{l a b}$ axis perpendicular to the static magnetic field $B_{0}$.

$$
H_{R F}=-\hbar \gamma B_{1} \cos \left(\omega_{r o t} t+\phi\right) I_{x}^{l a b}
$$

This Hamiltonian may then be transformed into the rotating frame, as in equation 2.31 and the new RF Hamiltonian is shown below. 


$$
\begin{aligned}
\tilde{H}_{R F} & =-\frac{\hbar \gamma B_{1}}{2}\left[I_{x} \cos \phi-I_{y} \sin \phi\right]- \\
& \frac{\hbar \gamma B_{1}}{2}\left[\cos \left(2 \omega_{r o t} t+\phi\right) I_{x}-\sin \left(2 \omega_{r o t} t+\phi\right) I_{y}\right] \\
& \approx-\frac{\hbar \gamma B_{1}}{2}\left[I_{x} \cos \phi-I_{y} \sin \phi\right]
\end{aligned}
$$

This shows how the single oscillating RF field is converted into two RF fields, one at zero frequency and the other at $2 \omega_{\text {rot }}$. In addition, this shows that by using a standard single coil in the laboratory frame, fully one half of the useful RF power is lost in the rotating frame. This second field averages to zero in the interaction frame and cannot affect the density matrix, just as a high frequency oscillating magnetic field cannot pick up a piece of iron in the laboratory frame. The rotating frame RF Hamiltonian is now the dominant Hamiltonian in the interaction frame, as long as the offset, $\left(\omega_{r o t}-\omega_{l}\right)$, is small compared to $\frac{\gamma B_{1}}{2}$. Now suppose we allow the equilibrium density matrix to evolve for a time $\tau$ under the RF Hamiltonian (assuming no offset and $\phi=0$ ). In this case, the density matrix after a pulse will be described by the following equation.

$$
\begin{aligned}
\rho(\tau) & =e^{-i \omega_{1} \tau I_{x}} \rho(0) e^{i \omega_{1} \tau I_{x}} \\
& =e^{-i \omega_{1} \tau I_{x}} I_{z} e^{i \omega_{1} \tau I_{x}} \\
& =I_{z} \cos \omega_{1} \tau-I_{y} \sin \omega_{1} \tau
\end{aligned}
$$

This shows the same features as the classical description. In fact, including a phase or offset term leads to identical results as the classical results. As a final note on the RF Hamiltonian, by controlling the phase $\phi$ of the RF, the researcher can effectively apply fields along both the $x_{r o t}$ and $y_{r o t}$ axes, which proves essential in the case of multiple pulse experiments.

\section{Chemical Shift Anisotropy}

The chemical shift interaction is a good starting point to look at the major features of the internal NMR Hamiltonians. Chemical shifts arise from the interaction of the magnetic dipole moment of the nucleus and local magnetic fields generated by both the 
motion of electrons in the large magnetic field (diamagnetic effects) and paramagnetic effects due to excited state electrons ${ }^{22}$. I will not discuss the theoretical schemes used to calculate these shifts and will suffice it to say that they exist and are often anisotropic (dependent on orientation of the molecule). The form of the chemical shift Hamiltonian is given below in equation $2.35 \mathrm{a}$.

$$
\begin{aligned}
H_{C S A} & =\hbar \gamma \delta_{i s o, c s} B_{0} I_{0}+\hbar \omega_{c s a} \sum_{m=-2}^{2}(-1)^{m} A_{2,-m}^{C S} T_{2, m}^{C S} \\
\omega_{c s a} & =\sqrt{\frac{2}{3}} \gamma \delta^{C S} \\
T_{2,0}^{C S} & =B_{0} I_{0} \\
T_{2, \pm 1}^{C S} & =B_{0} I_{ \pm 1} \\
T_{2, \pm 2}^{C S} & =0
\end{aligned}
$$

Where $A_{2, m}^{C S}$ is defined below.

$$
\begin{aligned}
& A_{2, m}^{C S}=\sum_{m^{\prime}=-2}^{2} D_{m^{\prime}, m}^{(2)}\left(\alpha^{C S}, \beta^{C S}, \gamma^{C S}\right) \rho_{2, m^{\prime}}^{C S} \\
& \delta^{C S}=\left(\delta_{z z}-\delta_{i s o, c s}\right) \\
& \rho_{2,0}^{C S}=\sqrt{\frac{3}{2}} \\
& \rho_{2, \pm 1}^{C S}=0 \\
& \rho_{2, \pm 2}^{C S}=\frac{1}{2} \eta_{C S}=\frac{\left(\delta_{x x}-\delta_{y y}\right)}{2\left(\delta_{z z}-\delta_{i s o, c s}\right)}
\end{aligned}
$$

The principal values of the chemical shift tensor (sometimes reported instead of $\delta_{i s o, c s}, \delta^{C S}$ and $\left.\eta_{C S}\right)$ are arranged such that $\left|\delta_{z z}-\delta_{i s o, c s}\right| \geq\left|\delta_{y y}-\delta_{i s o, c s}\right| \geq\left|\delta_{x x}-\delta_{\text {iso,cs }}\right|$. The values of these principal values are very small and are usually reported in units of parts per million (ppm). For ${ }^{13} \mathrm{C}$, for instance, the range of possible chemical shifts is from about -20 to $250 \mathrm{ppm}$ which is about $27 \mathrm{kHz}$ at a Larmor frequency of $100 \mathrm{MHz}$. The Euler angles, $\left(\alpha^{C S}, \beta^{C S}, \gamma^{C S}\right)$, refer to the orientation of the principal axis system of the chemical shift tensor relative to the laboratory frame (see definition of Euler angles in 
figure 2.1). This Hamiltonian, while appearing quite complex on the outside, actually can be greatly reduced when transformed to the rotating frame.

$$
\begin{aligned}
\tilde{H}_{C S A} & =\hbar \gamma \delta_{i s o, c s} B_{0} I_{0}+\hbar \omega_{c s a} \sum_{m=-2}^{2}(-1)^{m} A_{2,-m}^{C S} \tilde{T}_{2, m}^{C S} \\
\tilde{T}_{2,0}^{C S} & =B_{0} I_{0} \\
\tilde{T}_{2,+1}^{C S} & =B_{0}\left(I_{+1} \cos \omega_{r o t} t-i I_{-1} \sin \omega_{r o t} t\right) \\
\tilde{T}_{2,-1}^{C S} & =B_{0}\left(I_{-1} \cos \omega_{r o t} t-i I_{+1} \sin \omega_{r o t} t\right)
\end{aligned}
$$

In the rotating frame Hamiltonian, all of the oscillating terms, $\tilde{T}_{2, \pm 1}^{C S}$, may be ignored (alternatively an identical result comes from first-order perturbation theory), leaving the simple chemical shift Hamiltonian.

$$
\tilde{H}_{C S A}=\hbar \delta_{i s o, c s} \omega_{l} I_{0}+\hbar \omega_{c s a} B_{0} A_{2,0}^{C S} I_{0}
$$

Another way of thinking about the truncating effect of transforming to the rotating frame is to say that any terms in any Hamiltonian which do not commute with the Zeeman Hamiltonian will oscillate rapidly in the rotating frame and average to zero. In terms of energy level splittings, the chemical shift may be expressed below.

$$
\begin{aligned}
\Delta E_{m \rightarrow m-1}^{C S A} & =\left\langle I, m\left|\tilde{H}_{C S A}\right| I, m\right\rangle-\left\langle I, m-1\left|\tilde{H}_{C S A}\right| I, m-1\right\rangle \\
& =\hbar \omega_{l}\left(\delta_{i s o, c s}+\sqrt{\frac{2}{3}} \delta^{C S} A_{2,0}^{C S}\right)
\end{aligned}
$$

\section{Quadrupolar Interaction}

The first discussion of the quadrupolar interaction was by Casimir in an essay on the nuclear-electric hyperfine interaction in $1936 .{ }^{23}$ Additionally, one of the more complete early treatments of the quadrupolar interaction is the work by Cohen and Reif. ${ }^{24}$ More modern information on this subject may be found in additional sources. ${ }^{6,12,15}$ The basic Hamiltonian has the same form as the chemical shift anisotropy Hamiltonian. 


$$
\begin{aligned}
H_{Q} & =\hbar \omega_{Q} \sum_{m=-2}^{2}(-1)^{m} A_{2,-m}^{Q} T_{2, m}^{Q} \\
\omega_{Q} & =\frac{e^{2} q Q}{2 I(2 I-1) \hbar} \\
T_{2,0}^{Q} & =\frac{1}{\sqrt{6}}\left(3 I_{0}^{2}-I^{2}\right) \\
T_{2, \pm 1}^{Q} & =\frac{1}{\sqrt{2}}\left(I_{0} I_{ \pm 1}+I_{ \pm 1} I_{0}\right) \\
T_{2, \pm 2}^{Q} & =I_{ \pm 1}^{2}
\end{aligned}
$$

Where $A_{2, m}^{Q}$ is defined below.

$$
\begin{aligned}
A_{2, m}^{Q} & =\sum_{m^{\prime}=-2}^{2} D_{m^{\prime}, m}^{(2)}\left(\alpha^{Q}, \beta^{Q}, \gamma^{Q}\right) \rho_{2, m^{\prime}}^{Q} \\
e q & =V_{z z} \\
\rho_{2,0}^{Q} & =\sqrt{\frac{3}{2}} \\
\rho_{2, \pm 1}^{Q} & =0 \\
\rho_{2, \pm 2}^{Q} & =\frac{1}{2} \eta_{Q}=\frac{\left(v_{x x}-V_{y y}\right)}{2 V_{z z}}
\end{aligned}
$$

Again, as in the previous section, the values of the electric field gradient (EFG) in the principal axis frame are defined such that $\left|V_{z z}\right| \geq\left|V_{y y}\right| \geq\left|V_{x x}\right|$ and the Euler angles refer to the orientation of the EFG axis system relative to the laboratory frame. Additionally, since the quadrupolar tensor is traceless, there is no net isotropic shift due to this interaction (at least to first order in perturbation theory). As before, we can truncate this Hamiltonian by eliminating the non-commuting $\left(\tilde{T}_{2, \pm 1}^{Q}, \tilde{T}_{2, \pm 2}^{Q}\right)$ terms. The remaining Hamiltonian is expressed in equation 2.41 .

$$
\tilde{H}_{Q}=\frac{\hbar \omega_{Q}}{\sqrt{6}} A_{2,0}^{Q}\left(3 I_{0}^{2}-I(I+1)\right)
$$

This first-order perturbation result illustrates an example where higher order corrections are needed. To show the necessity, we look at the eigenvalues for the $2 I+1$ energy levels of the Zeeman basis states. 


$$
\begin{aligned}
\left\langle I, m\left|\tilde{H}_{Q}\right| I, m\right\rangle & =E_{m}^{(1 Q)}=\frac{\hbar \omega_{Q}}{\sqrt{6}} A_{2,0}^{Q}\left\langle I, m\left|\left(3 I_{0}^{2}-I(I+1)\right)\right| I, m\right\rangle \\
E_{m}^{(1 Q)} & =\frac{\hbar \omega_{Q}}{\sqrt{6}} A_{2,0}^{Q}\left(3 m^{2}-I(I+1)\right) \\
& \vdots \\
E_{0}^{(1 Q)} & =-\frac{\hbar \omega_{Q}}{\sqrt{6}} A_{2,0}^{Q}(I(I+1)) \\
& \text { or } \\
E_{ \pm \frac{1}{2}}^{(1 Q)} & =\frac{\hbar \omega_{Q}}{\sqrt{6}} A_{2,0}^{Q}\left(\frac{3}{4}-I(I+1)\right)
\end{aligned}
$$

The last two energies are for the two distinct cases where the spin is either an integer $(I=1,2,3, \ldots)$ or half-odd integer $\left(I=\frac{1}{2}, \frac{3}{2}, \frac{5}{2}, \ldots\right)$. Also, it is notable that in the spin $1 / 2$ case, only the final $E_{ \pm \frac{1}{2}}^{(1 Q)}$ energy levels exist and are analytically zero, as is expected since a spin $1 / 2$ has no quadrupolar moment of the nucleus. At this point it is valuable to calculate the energy splittings between a variety of single quantum $(\Delta m= \pm 1)$ transitions.

$$
\Delta E_{m \rightarrow m-1}^{(1 Q)}=E_{m}^{(1 Q)}-E_{m-1}^{(1 Q)}=\frac{3 \hbar \omega_{Q}}{\sqrt{6}} A_{2,0}^{Q}(2 m-1)
$$

The first feature which is immediately visible is that for $m=\frac{1}{2}$, the splitting is analytically zero. In fact for any other value of $m$, this splitting will depend on both the size of the quadrupolar coupling and the orientation of a given crystallite. Only half-odd integer spins have a central transition $\left(\frac{1}{2} \leftrightarrow-\frac{1}{2}\right)$ which is unaffected by the quadrupolar interaction to first order. In addition, if we calculate the energy splitting for a multiple quantum ( $m \leftrightarrow-m$ ) transition (for both integer and half-odd integer spins), we find that these also have no first-order quadrupolar energy splitting correction. For both the central transition and multiple quantun $\mathrm{n}_{\mathrm{t}}$ transition, it is crucial to calculate the second-order contribution to the energy splitting since this becomes the dominant energy splitting correction. Additionally, when the quadrupolar coupling is large, the second-order quadrupolar correction will make significant changes to the satellite transition energy splittings. For the remainder of this thesis, I will describe experiments and theory pertaining primarily to the central transition of half-odd integer spins. For more information on the study of multi- 
ple-quantum overtone spectroscopy or integer spin spectroscopy, I direct the reader to the original NMR references and references therein. ${ }^{6}, 7,12,25-28$

To calculate the second order correction to the energy splittings in a quadrupolar system, we need to return to equation 2.18 where now the sum is over the additional $2 I$ Zeeman states (all except for the $m$ state) for a quadrupolar spin $I$.

$$
E_{m}^{(2 Q)}=\sum_{n \neq m} \frac{\left\langle I, m\left|H_{Q}\right| I, n\right\rangle\left\langle I, n\left|H_{Q}\right| I, m\right\rangle}{\hbar \omega_{l}(m-n)}
$$

Rather than calculate this for a general $m$, I will instead look only at the central transition second-order quadrupolar energy splitting expression.

$$
\begin{aligned}
& \Delta E_{\frac{1}{2} \rightarrow-\frac{1}{2}}^{(2 Q)}=\sum_{n \neq \frac{1}{2}} \frac{\left\|\left\langle I, n\left|H_{Q}\right| I, \frac{1}{2}\right\rangle\right\|^{2}}{\hbar \omega_{l}\left(\frac{1}{2}-n\right)}-\sum_{n \neq-\frac{1}{2}} \frac{\left\|\left\langle I, n\left|H_{Q}\right| I,-\frac{1}{2}\right\rangle\right\|^{2}}{\hbar \omega_{l}\left(-\frac{1}{2}-n\right)}
\end{aligned}
$$

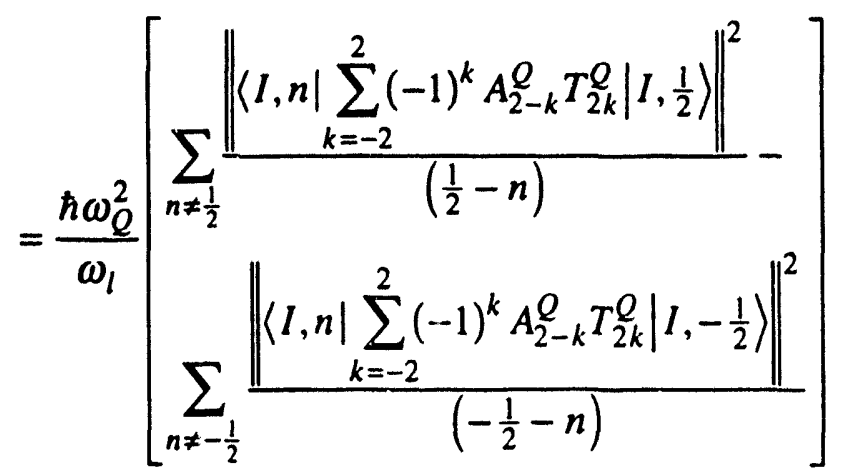

These sums may be simplified by realizing that the $T_{2 k}^{Q}$ spin operators produce non-zero results for only very specific bra-ket pairs. For example, $\left\langle I, j\left|T_{20}^{Q}\right| I, m\right\rangle \neq 0$ only when $j$ and $m$ are the same. In fact, below are shown the only non-zero matrix elements involving the $\left| \pm \frac{1}{2}\right\rangle$ ket, which will be used to simplify equation 2.45 .

$$
\begin{aligned}
\left\langle I, \pm \frac{1}{2}\left|T_{20}^{Q}\right| I, \pm \frac{1}{2}\right\rangle & =\frac{1}{\sqrt{6}}\left(\frac{3}{4}-I(I+1)\right) \\
\left\langle I, \mp \frac{1}{2}\left|T_{2 \pm 1}^{Q}\right| I, \pm \frac{1}{2}\right\rangle & =0 \\
\left\langle I, \pm \frac{3}{2}\left|T_{2 \pm 1}^{Q}\right| I, \pm \frac{1}{2}\right\rangle & =\sqrt{\left(I-\frac{1}{2}\right)\left(I+\frac{3}{2}\right)} \\
\left\langle I, \mp \frac{3}{2}\left|T_{2 \pm 2}^{Q}\right| I, \pm \frac{1}{2}\right\rangle & =\frac{\left(I+\frac{1}{2}\right)}{2} \sqrt{\left(I-\frac{1}{2}\right)\left(I+\frac{3}{2}\right)} \\
\left\langle I, \pm \frac{5}{2}\left|T_{2 \pm 2}^{Q}\right| I, \pm \frac{1}{2}\right\rangle & =\frac{1}{2} \sqrt{\left(I-\frac{1}{2}\right)\left(I-\frac{3}{2}\right)\left(I+\frac{3}{2}\right)\left(I+\frac{5}{2}\right)}
\end{aligned}
$$


Also, because the sums over $n$ are limited to $n \neq+\frac{1}{2}$ and $n \neq-\frac{1}{2}$ respectively, the first of these relationships will not be used at all. The second shows that in fact all $n \neq \pm \frac{1}{2}$ may be ignored. Additionally, if the spin is $I=3 / 2$, then the final relationship will also disappear (as evident in the fourth formula of 2.46 and in the fact that there is no $5 / 2$ state for a spin $3 / 2$ nucleus). Now performing the sums in equation 2.45 we arrive at the following formula for the second-order central transition energy splitting correction. Equation 2.47 was simplified using the complex conjugate relationship $\left(.1 \frac{Q}{2 m}\right)^{\dagger}=(-1)^{m} A_{2-m}^{Q}$ which is correct in this case, while not generally true for all tensors.

$$
\Delta E_{\frac{1}{2} \rightarrow-\frac{1}{2}}^{(2 Q)}=\frac{\hbar \omega_{Q}^{2}}{\omega_{l}}\left(I(I+1)-\frac{3}{4}\right)\left(2 A_{21}^{Q} A_{2-1}^{Q}+A_{22}^{Q} A_{2-2}^{Q}\right)
$$

This result is now in a form which may be used to calculate actual NMR line positions, which we will do in the next section.

\section{Dipole-Dipole Interaction}

The homonuclear and heteronuclear dipolar coupling Hamiltonians are some of the most well studied in NMR. The basic form for a Hamiltonian describing the coupling between two spins $i$ and $j$ is given in equation 2.48 .

$$
\begin{aligned}
H_{D} & =-\sum_{j \neq i} \hbar \omega_{D_{i j}} \sum_{m=-2}^{2}(-1)^{m} A_{2,-m}^{D_{i j}} T_{2, m}^{D_{i j}} \\
\omega_{D_{i j}} & =\frac{\gamma_{i} \gamma_{j}}{r_{i, j}^{3}} \\
T_{2,0}^{D_{i j}} & =\frac{1}{\sqrt{6}}\left(3 I_{i, 0} I_{j, 0}-I_{i} \cdot I_{j}\right) \\
T_{2, \pm 1}^{D_{i j}} & =\frac{1}{\sqrt{2}}\left(I_{i, 0} I_{j, \pm 1}+I_{i, \pm 1} I_{j, 0}\right) \\
T_{2, \pm 2}^{D_{i j}} & =I_{i, \pm 1} I_{j, \pm 1}
\end{aligned}
$$

Where $A_{2, m}^{D_{i j}}$ is defined below. 


$$
\begin{aligned}
A_{2, m}^{D_{i j}} & =\sum_{m^{\prime}=-2}^{2} D_{m^{\prime}, m}^{(2)}\left(\alpha_{i j}^{D}, \beta_{i j}^{D}, \gamma_{i j}^{D}\right) \rho_{2, m^{\prime}}^{D_{i j}} \\
\rho_{2,0}^{D_{i j}} & =\sqrt{\frac{3}{2}} \\
\rho_{2, \pm 1}^{D_{i j}} & =\rho_{2, \pm 2}^{D_{i j}}=0
\end{aligned}
$$

As usual, the Euler angles refer to the orientation of the principal axis of the dipolar coupling (corresponding to the vector connecting the two nuclei) relative to the laboratory frame. It is immediately apparent upon substitution of the principal axis components into the formula for the spatial tensor $A_{2, m}^{D_{i j}}$, that only $\beta_{i j}^{D}$, which corresponds to the angle between the internuclear vector and the static magnetic field, affects the overall Hamiltonian. Secondly, truncation of this Hamiltonian by the Zeeman Hamiltonian is more difficult because there are two cases, the homonuclear ( $i$ and $j$ have similar Larmor frequencies) and the heteronuclear ( $i$ and $j$ have different Larmor frequencies). In any case, only the $m=0$ term will remain, giving the following Hamiltonian.

$$
H_{D}=-\sum_{j \neq i} \hbar \omega_{D_{i j}} \frac{1}{2}\left(3 I_{i, 0} I_{j, 0}-I_{i} \cdot I_{j}\right) d_{0,0}^{(2)}\left(\beta_{i j}^{D}\right)
$$

I will return to this formula in chapter 3 when discussing the homonuclear dipolar coupling contribution to dynamic-angle spinning linewidth. In addition, I will use this Hamiltonian when discussing the theory of cross-polarization in chapter 5. In all other cases, the dipolar coupling may be thought of as an irreversible relaxation mechanism which leads to a Gaussian decay.

\section{Truncation of RF Hamiltonian by the Quadrupolar Hamiltonian}

The quadrupolar Hamiltonian is often times the second largest interaction present in a system (in most cases only the Zeeman is larger) even in the presence of RF irradiation. As such, when both radio frequency irradiation and quadrupolar Hamiltonians are present, it is important to evaluate the relative sizes of these two parts, ${ }^{29-34}$ just as the Zeeman offset term affects the RF effectiveness. To compare the RF and quadrupolar 
Hamiltonians, we need only examine the first-order contributions, since the second-order quadrupolar interaction will rarely be larger than $100 \mathrm{kHz}$ in the systems we study. The total RF and quadrupolar Hamiltonian which is present in the rotating frame is given below.

$$
\begin{aligned}
\tilde{H} & =\tilde{H}_{Z}+\tilde{H}_{Q}+\tilde{H}_{R F} \\
& =\hbar\left(\omega_{l}-\omega_{r o t}\right) I_{0}+\frac{\hbar \omega_{Q}}{\sqrt{6}} A_{2,0}^{Q}\left(3 I_{0}^{2}-I(I+1)\right) \\
& +\frac{\hbar \gamma B_{1}}{2}\left[I_{x} \cos \phi-I_{y} \sin \phi\right]
\end{aligned}
$$

In order to simplify this Hamiltonian, we may rewrite it in terms of fictitious spin 1/2 operators. These are spin operators which involve only two states and look like the traditional spin $1 / 2$ operators. Given below are the basic definitions which have been outlined previously. ${ }^{25,26,35}$

$$
\begin{array}{ll}
I_{z}^{j k}=\frac{1}{2}(|j\rangle\langle j|-| k\rangle\langle k|) & I_{z}^{j k}=-I_{z}^{k j} \\
I_{x}^{j k}=\frac{1}{2}(|j\rangle\langle k|+| k\rangle\langle j|) & I_{x}^{j k}=I_{x}^{k j} \\
I_{y}^{j k}=\frac{i}{2}(-|j\rangle\langle k|+| k\rangle\langle j|) & I_{y}^{j k}=-I_{y}^{k j}
\end{array}
$$

Within the $j k$ manifold, these operators obey the usual spin $1 / 2$ commutation rules

$$
\left[I_{\alpha}^{j k}, I_{\beta}^{j k}\right]=i I_{\gamma}^{j k}
$$

where $\alpha, \beta, \gamma=x, y, z$ and cyclic permutations. For commutators between different submanifolds, the following commutation rules apply.

$$
\begin{array}{ll}
{\left[I_{x}^{j k}, I_{x}^{i k}\right]=\left[I_{y}^{j k}, I_{y}^{i k}\right]=\frac{i}{2} I_{y}^{j i}} \\
{\left[I_{z}^{j k}, I_{z}^{i k}\right]=0} & {\left[I_{x}^{j k}, I_{y}^{i k}\right]=\frac{i}{2} I_{x}^{j i}} \\
{\left[I_{x}^{j k}, I_{z}^{i k}\right]=\frac{-i}{2} I_{y}^{j k}} & {\left[I_{y}^{j k}, I_{z}^{i k}\right]=\frac{i}{2} I_{x}^{j k}} \\
{\left[I_{\alpha}^{j k}, I_{\beta}^{i l}\right]=0} &
\end{array}
$$

The last is the most important, since this states that all fictitious spin-1/2 operators commute between unconnected manifolds. Using these relationships, the normal $I_{z}, I_{x}$ and $I_{y}$ 
may be redefined in the Hamiltonian given by equation 2.48 (where the spin $I$ is a halfodd integer spin) using the following relationships.

$$
\begin{aligned}
I_{z} & =2 I\left(I_{z}^{1,2}+I_{z}^{2 I, 2 I+1}\right)+2(2 I-1)\left(I_{z}^{2,3}+I_{z}^{2 I-1,2 I}\right)+\cdots \\
& +n(2 I-(n-1))\left(I_{z}^{n, n+1}+I_{z}^{2 I+1-n, 2 I+2-n}\right)+\cdots \\
& +\left(I+\frac{1}{2}\right)^{2}\left(I_{z}^{\left.\frac{2 l+1}{2}, \frac{2 I+3}{2}\right)}\right. \\
I_{x} & =\sqrt{2 I}\left(I_{x}^{1,2}+I_{x}^{2 I, 2 I+1}\right)+\sqrt{2(2 I-1)}\left(I_{x}^{2,3}+I_{x}^{2 I-1,2 I}\right)+\cdots \\
& +\sqrt{n(2 I-(n-1))}\left(I_{x}^{n, n+1}+I_{x}^{2 l+1-n, 2 I+2-n}\right)+\cdots \\
& +\left(I+\frac{1}{2}\right)\left(I_{x}^{\frac{2 l+1}{2}, \frac{2 l+3}{2}}\right) \\
I_{y} & =\sqrt{2 I}\left(I_{y}^{1,2}+I_{y}^{2 l, 2 I+1}\right)+\sqrt{2(2 I-1)}\left(I_{y}^{2,3}+I_{y}^{2 I-1,2 I}\right)+\cdots \\
& +\sqrt{n(2 I-(n-1))}\left(I_{y}^{n, n+1}+I_{y}^{2 I+1-n, 2 I+2-n}\right)+\cdots \\
& +\left(I+\frac{1}{2}\right)\left(I_{y}^{\frac{2 l+1}{2}, \frac{2 l+3}{2}}\right)
\end{aligned}
$$

Additionally, the $\left(3 I_{0}^{2}-I(I+1)\right)$ term may also be reduced.

$$
\begin{aligned}
\left(3 I_{0}^{2}-I(I+1)\right)= & \frac{1}{2}\left(2 I^{2}-I\right)\left(I_{z}^{1,2}-I_{z}^{2 I, 2 I+1}\right)+\frac{1}{2}\left(4 I^{2}-8 I+3\right)\left(I_{z}^{2,3}-I_{z}^{2 I-1,2 I}\right) \\
& +\cdots+\frac{1}{2}\left(2 n I^{2}-\sum_{i=0}^{n-1}(6 i+1) I+3 \sum_{i=0}^{n-1} i^{2}\right)\left(I_{z}^{n, n+1}-I_{z}^{2 I+1-n, 2 I+2-n}\right)(2.5 \\
& +\cdots+(0)\left(I_{z}^{\frac{2 l+1}{2}, \frac{2 l+3}{2}}\right)
\end{aligned}
$$

The last term is of particular importance, as this is the central transition contribution of the first-order quadrupolar Hamiltonian. As expected, this is zero and this makes the central transition manifold different from all of the other fictitious spin 1/2 manifolds for this Hamiltonian. The Hamiltonian from equation 2.51 may be divided into a sum of $j k$ sub-manifold Hamiltonians $\tilde{H}^{j k}$ below with all other Hamiltonian elements $(j-k \neq \pm 1)$ zero 


$$
\begin{aligned}
\tilde{H}^{j, j+1}+\tilde{H}^{n, n+1}= & \hbar j\left(\omega_{l}-\omega_{r o l}\right)(2 I-j+1)\left(I_{z}^{j, j+1}+I_{z}^{n, n+1}\right)+ \\
& \frac{\hbar \omega_{Q}}{2 \sqrt{6}} A_{2,0}^{Q}\left(2 j I^{2}+\sum_{i=0}^{j-1}\left(3 i^{2}-(6 i+1) I\right)\right)\left(I_{z}^{j, j+1}-I_{z}^{n, n+1}\right)+ \\
& \frac{\hbar \gamma B_{1}}{2} \sqrt{j(2 I-j+1)}\left[\begin{array}{l}
\left(I_{x}^{j, j+1}+I_{x}^{n, n+1}\right) \cos \phi- \\
\left(I_{y}^{j, j+1}+I_{y}^{n, n+1}\right) \sin \phi
\end{array}\right]
\end{aligned}
$$

where we define $n=2 I+1-j$. These Hamiltonians may be truncated to first-order using our knowledge about the size of the interactions, $\left(\omega_{l}-\omega_{\text {rot }}\right)<\frac{\gamma B_{1}}{2}<<\frac{\omega_{Q}}{2 \sqrt{6}} A_{2,0}^{Q}$.

$$
\begin{aligned}
\tilde{H}^{j, j+1}+\tilde{H}^{n, n+1}= & \hbar j\left(\omega_{l}-\omega_{\text {rot }}\right)(2 I-J+1)\left(I_{z}^{j, j+1}+I_{z}^{n, n+1}\right)+ \\
& \frac{\hbar \omega_{Q}}{2 \sqrt{6}} A_{2,0}^{Q}\left(2 j I^{2}+\sum_{i=0}^{j-1}\left(3 i^{2}-(6 i+1) I\right)\right)\left(I_{z}^{j, j+1}-I_{z}^{n, n+1}\right) \\
\tilde{H}^{\frac{2 l+1}{2}, \frac{2 l+3}{2}}= & \hbar\left(\omega_{l}-\omega_{r o t}\right)\left(I+\frac{1}{2}\right)^{2} I_{z}^{\frac{2 l+1}{2}, \frac{2 l+3}{2}+} \\
& \frac{\hbar \gamma B_{1}}{2}\left(I+\frac{1}{2}\right)\left[I_{x}^{\frac{2 l+1}{2}, \frac{2 l+3}{2}} \cos \phi-I_{y}^{\frac{2 l+1}{2}, \frac{2 l+3}{2}} \sin \phi\right]
\end{aligned}
$$

This shows that the application of an RF pulse to a quadrupolar system produces distinctively different results that when applied to a spin $1 / 2$ system. First, the Hamiltonians under RF irradiation for the outer transition sub-manifolds (the upper equation in 2.55) are all identical and are equivalent to free precession without RF irradiation. Second, the Hamiltonian for the central transition (the lower equation in 2.55 ) corresponds to the same Hamiltonian as a spin $1 / 2$ under RF irradiation, with the change by a factor of $I+\frac{1}{2}$ in the effective rotating frame magnetic field. Thus, a $90^{\circ}$ pulse for a quadrupolar nucleus will be shorter than for a comparable spin $1 / 2$ system by a factor of $I+\frac{1}{2}$ since the RF precession frequency is scaled by this factor. The effect of quadrupolar truncation of the RF Hamiltonian will be more thoroughly discussed later when we look at cross-polarization of quadrupolar nuclei under Hartmann-Hahn matching conditions (see Chapter 5). 


\section{Coherence Pathways}

Having briefly described both the Hamiltonians present in most solid systems and the effect of truncation, it may now be useful to describe evolution and detection of NMR signals. For most of the experiments in this thesis, I will look at simple two level systems (either spin $1 / 2$ or the central transition of a half-odd integer quadrupolar nucleus). In the absence of dipolar coupling, there are only three different coherent states that the density matrix can evolve into or from; they are $I_{z}, I_{y}$ and $I_{x}$ (for quadrupolar nuclei, the central transition superscripts have been dropped). Alternatively, the density matrix may be expressed in terms of spherical tensor operators $I_{0}, I_{+1}$, and $I_{-1}$. Our detector will always be a single coil capable of collecting data in quadrature (both the real and imaginary components in the induced FID). Therefore the observable in our experiments will be $I_{+1}$ (or $I_{-1}$ but never both). However, the phase of the receiver $\left(\phi_{r}\right)$ may be controlled (by adding the real and imaginary data from each scan differently in the computer acquisition system to form the FID) to arbitrary accuracy in the computer. That is to say our observable may be written in equation 2.59 as

$$
S(t)=\operatorname{tr}\left[\rho(t) I_{+1} e^{-i \phi_{r}}\right] .
$$

This has the property of extracting only the $I_{-1}$ components from the density matrix; all other components will have a zero trace. The response of the system to pulses and free evolution can be characterized by the Hamiltonians below (where the pulse is applied with phase $\phi)$.

$$
\begin{array}{ll}
\tilde{H}_{f i d}=\hbar \omega_{f i d} I_{2} & \omega_{f i d}=\frac{\Delta E_{f i d}}{\hbar} \\
\tilde{H}_{R F}=\hbar \omega_{R F}\left[I_{x} \cos \phi-I_{y} \sin \phi\right] & \omega_{R F}=\frac{\gamma B_{1}}{2}\left(I+\frac{1}{2}\right)
\end{array}
$$

The response of each of the possible states of the density matrix to each Hamiltonian is given below. 


$$
\begin{gathered}
U_{R F}=\exp \left[-i \tilde{H}_{R F} t / \hbar\right]=\exp \left[-i \omega_{R F} t\left(I_{x} \cos \phi-I_{y} \sin \phi\right)\right] \\
U_{f i d}=\exp \left[-i \omega_{f i d} t I_{z}\right] \\
U_{R F} I_{z} U_{R F}^{\dagger}=I_{z} \cos \omega_{R F} t+\sin \omega_{R F} t\left[I_{x} \sin \phi-I_{y} \cos \phi\right] \\
U_{R F} I_{x} U_{R F}^{\dagger}=I_{x}\left(\cos ^{2} \phi+\sin ^{2} \phi \cos \omega_{R F} t\right)+I_{y} \frac{\sin 2 \phi}{2}\left(1-\cos \omega_{R F} t\right)- \\
I_{z} \sin \phi \sin \omega_{R F} t \\
U_{R F} I_{y} U_{R F}^{\dagger}=I_{y}\left(\sin ^{2} \phi+\cos ^{2} \phi \cos \omega_{R F} t\right)+I_{x} \frac{\sin 2 \phi}{2}\left(1-\cos \omega_{R F} t\right)+ \\
I_{z} \cos \phi \sin \omega_{R F} t \\
U_{f i d} I_{z} U_{f i d}^{\dagger}=I_{z} \\
U_{f i d} I_{x} U_{f i d}^{\dagger}=I_{x} \cos \omega_{f i d} t+I_{y} \sin \omega_{f i d} t \\
U_{f i d} I_{y} U_{f i d}^{\dagger}=I_{y} \cos \omega_{f i d} t-I_{x} \sin \omega_{f i d} t
\end{gathered}
$$

These equation are more useful when expressed in the spherical operator basis set.

$$
\begin{aligned}
& U_{R F} I_{0} U_{R F}^{\dagger}=I_{0} \cos \omega_{R F} t+\frac{i \sin \omega_{R F^{t}}}{\sqrt{2}}\left[I_{+1} e^{-i \phi}+I_{-1} e^{i \phi}\right] \\
& U_{R F} I_{ \pm 1} U_{R F}^{\dagger}=\frac{1}{2} I_{ \pm 1}\left(1+\cos \omega_{R F} t\right)-\frac{1}{2} I_{\mp 1}\left(1-\cos \omega_{R F} t\right) e^{ \pm 2 i \phi}+ \\
& \frac{i}{\sqrt{2}} I_{0} e^{ \pm i \phi} \sin \omega_{R F^{t}} \\
& U_{f i d} I_{0} U_{f i d}^{\dagger}=I_{0} \\
& U_{f i d} I_{ \pm 1} U_{f i d}^{\dagger}=I_{ \pm 1} e^{\mp i \omega_{f i d^{t}}}
\end{aligned}
$$

These equations may now be used to show the importance of coherence pathways in NMR. As an initial example, we will look at an experiment consisting of a $90^{\circ}$ pulse with phase $\phi_{1}$ followed by a delay $t_{1}$ followed by a $90^{\circ}$ pulse with phase $\phi_{2}$ followed by a delay $t_{1}$ and then acquisition with phase $\phi_{r}$. The observed signal may then be calculated using the above equations (2.62). Our initial density matrix will be $I_{0}$. This evolves during the first pulse into the following coherences (or states).

$$
I_{0} \stackrel{90_{\phi_{1}}}{\longrightarrow} \frac{i}{\sqrt{2}}\left(I_{+1} e^{-i \phi_{1}}+I_{-1} e^{i \phi_{1}}\right)
$$

This state will continue to evolve under the FID Hamiltonian for a period $t_{1}$ giving the following result. 


$$
\frac{i}{\sqrt{2}}\left(I_{+1} e^{-i \phi_{1}}+I_{-1} e^{i \phi_{1}}\right) \stackrel{F I D}{\longrightarrow} \frac{i}{\sqrt{2}}\left(I_{+1} e^{-i\left(\phi_{1}+\omega_{f i d} t_{1}\right)}+I_{-1} e^{i\left(\phi_{1}+\omega_{f i d} t_{1}\right)}\right)
$$

The $90^{\circ}$ pulse is then applied, which gives the result below.

$$
\left(\begin{array}{l}
\frac{i}{\sqrt{2}} I_{+1} e^{-i\left(\phi_{1}+\omega_{f d} d_{1}\right)} \\
+\frac{i}{\sqrt{2}} I_{-1} e^{i\left(\phi_{1}+\omega_{f f d} t_{1}\right)}
\end{array}\right) \stackrel{90_{\phi_{2}}}{\longrightarrow}\left(\begin{array}{l}
\frac{i}{2 \sqrt{2}} I_{+1}\left(e^{-i\left(\phi_{1}+\omega_{f i d} t_{1}\right)}-e^{i\left(\phi_{1}+\omega_{f u} t_{1}-2 \phi_{2}\right)}\right) \\
+\frac{i}{2 \sqrt{2}} I_{-1}\left(e^{i\left(\phi_{1}+\omega_{f u d} t_{1}\right)}-e^{-i\left(\phi_{1}+\omega_{f i d} t_{1}-2 \phi_{2}\right)}\right) \\
-\frac{1}{2} I_{0}\left(e^{-i\left(\phi_{1}+\omega_{f u} t_{1}-\phi_{2}\right)}+e^{i\left(\phi_{1}+\omega_{f d d} t_{1}-\phi_{2}\right)}\right)
\end{array}\right)
$$

This density matrix will then evolve for a second $t_{1}+t_{2}$ period, at which point we can calculate the observable signal in $t_{2}$ (as detection occurs from $t_{2}=0$ on). Since evolution under the FID Hamiltonian does not result in any transfer of coherence between different density matrix states (for example $I_{-1}$ to $I_{+1}$ ), the only coherence we need consider is the $I_{-1}$ contribution (all others produce a trace of zero with our observable).

$$
\begin{gathered}
\frac{i}{2 \sqrt{2}} I_{-1}\left(\begin{array}{l}
e^{i\left(\phi_{1}+\omega_{f f d} t_{1}\right)}- \\
e^{-i\left(\phi_{1}+\omega_{f i d} t_{1}-2 \phi_{2}\right)}
\end{array}\right) \stackrel{F I D}{\longrightarrow} \frac{i}{2 \sqrt{2}} I_{-1} e^{i \omega_{f i d} t_{2}}\left(\begin{array}{l}
e^{i\left(\phi_{1}+2 \omega_{f j d} t_{1}\right)}- \\
e^{-i\left(\phi_{1}-2 \phi_{2}\right)}
\end{array}\right) \\
S\left(t_{1}, t_{2}\right)=\frac{i}{2 \sqrt{2}}\left(\begin{array}{l}
e^{2 i \omega_{f i d} t_{1}} e^{i\left(\phi_{1}-\phi_{r}\right)}- \\
e^{-i\left(\phi_{1}-2 \phi_{2}+\phi_{r}\right)}
\end{array}\right) e^{i \omega_{f f l} t_{2}} \operatorname{tr}\left[I_{-1} I_{+1}\right] \\
=-\frac{i}{4 \sqrt{2}}\left(\begin{array}{l}
e^{2 i \omega_{f d} t_{1}} e^{i\left(\phi_{1}-\phi_{r}\right)}- \\
e^{-i\left(\phi_{1}-2 \phi_{2}+\phi_{r}\right)}
\end{array}\right) e^{i \omega_{f u l} t_{2}}
\end{gathered}
$$

This signal shows two components. The upper component is the signal coming from direct evolution after the initial $90^{\circ}$ pulse (notice that the $t_{1}$ dependence is identical to one where there is no second pulse). The lower component is the echo signal (notice it has no $t_{1}$ dependence) coming from the second pulse. If we try to acquire a spectrum which contains only the echo signal, using only a single set of pulse and receiver phases will not cancel the unwanted component. Suppose now, we add together the signal from four different experiments where the phases of the pulses and receiver $\left(\phi_{1}, \phi_{2}, \phi_{r}\right)$ consist of the 
following four sets $\{(0,0,0),(90,0,270),(180,0,180),(270,0,90)\}$ (given in units of degrees). Adding together the four signals will produce the following result.

$$
\begin{aligned}
S\left(t_{1}, t_{2}\right) & =-\frac{i}{4 \sqrt{2}} e^{i \omega_{f i d} t_{2}}\left(\begin{array}{l}
e^{2 i \omega_{f i d} t_{1}}\left(e^{0}+e^{-i \pi}+e^{0}+e^{i \pi}\right)- \\
\left(e^{0}+e^{-2 i \pi}+e^{-2 i \pi}+e^{-2 i \pi}\right)
\end{array}\right) \\
& =\frac{i}{\sqrt{2}} e^{i \omega_{f i d} t_{2}}=\frac{1}{\sqrt{2}}\left(-\sin \omega_{f i d} t_{2}+i \cos \omega_{f i d} t_{2}\right)
\end{aligned}
$$

These phases choose only the coherence pathway that we desire, that is the echo signal. The phase cycle was arrived at by setting the net phase of the evolved signal equal to zero, which for this sequence was $\phi_{1}-2 \phi_{2}+\phi_{r}=0$. This equation tells us the nathe-

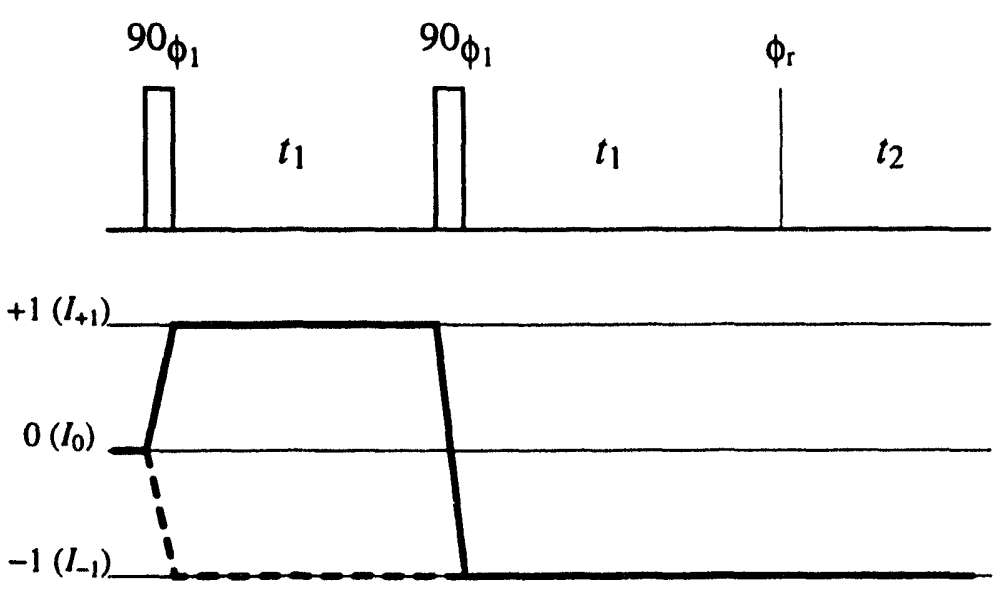

Figure 2.2 90-90 Echo Coherence Pathway. The bold line is the desired signal. The dashed line is the main artifact we wish to remove.

matical relationship between the phases. However, it does not tell us how many different phases each pulse must be cycled through to remove artifacts. This is actually an old and difficult question which I will not answer completely. The partial answer to this question can be seen in the formula for the signal. The upper signal came from a density matrix element that is proportional to $I_{-1}$ in both $t_{1}$ periods while the lower signal came from a density matrix that was proportional to $I_{+1}$ in the first $t_{1}$ period and $I_{-1}$ in the second. Graphically, this is shown in figure 2.2 where the bold line denotes the coherence pathway of the echo signal and the dashed line represents the coherence pathway of the un- 
wanted one pulse signal. This formalism was first put forth by both $\mathrm{Bain}^{36}$ and Bodenhausen et al. ${ }^{37}$ and is discussed quite thoroughly in Ernst's book on multidimensional NMR. ${ }^{7}$ In figure 2.2, the density matrix elements are labeled as coherences, in this case $+1,0$ and -1 ; these are often assigned the variable name $p$ and a transfer of coherence between levels is written as $\Delta p$. The coherence pathway for an experiment may be written as a vector $\Delta \mathrm{p}=\left(\Delta p_{1}, \Delta p_{2}, \Delta p_{3}, \ldots, \Delta p_{n}\right)$ of $n \Delta p_{i}$ elements where $n$ is the number of pulses. The $90^{\circ}-90^{\circ}$ echo experiment may be written $(+1,-2)$. It has been shown in the above references that when a pulse is independently cycled through $m$ phases $\left(0, \frac{2.1 \pi}{m}, \frac{2.2 \pi}{m}, \cdots, \frac{2(m-1) \pi}{m}\right)$, this cycle leads to selection the selection of a specific $\Delta p_{i}$ and additionally $\Delta p_{i} \pm(m-1), \Delta p_{i} \pm 2(m-1)$, etc. In our experiment, the quadrature of our receiver will automatically select only the -1 pathway in $t_{2}$. Therefore by guaranteeing that the first evolution period is $a+1$ coherence, we can assure that we observe only the signal we want. It is obvious that using only 2 phases for the first pulse will leave both $\Delta p_{1}$ of $+3,+1,-3$ and -1 . The +3 and -3 coherence transfers are harmless since the density matrix can only have coherence between +1 and -1 for our system. The -1 coherence transfer is a serious problem and indicates that the unwanted $(-1,0)$ pathway will survive. However, a phase cycle of 3 will result in $\Delta p_{1}$ of $+4,+1$, and -2 . None of the unwanted ( +4 and -2$)$ coherence transfers will produce any observable signal. In the phase cycle for the experiment given earlier, we could have used

$$
\{(0,0,0),(120,0,240),(240,0,120)\}
$$

however this would necessitate $120^{\circ}$ phase shifts which can be difficult on some spectrometers (especially in the receiver phase cycle). This same sort of analysis can be applied to more complex pulse sequences and at the appropriate locations, I will point out the reasoning behind the phase cycles for the experiments used in this thesis. For further information on phase cycling, the previously mentioned references will provide a good starting point as well as additional references contained therein. 


\section{Eigenvalues from static samples}

The eigenvalues from both the chemical shift and quadrupolar Hamiltonians have already been discussed. However, all of our expressions are in terms of zero and second rank spatial tensors $A_{l m}^{\lambda}$. These spatial tensors are highly dependent on the orientation of the principal axis system (PAS) of a crystallite with respect to the magnetic field (see equations 2.35 and 2.40. Under static (time-independent) conditions, both the quadrupolar and chemical shift energy levels can be calculated explicitly as functions of PAS orientation. Looking first at the chemical shift interaction, we expand $A_{20}^{C S}$ below.

$$
\begin{aligned}
& A_{20}^{C S}=\sum_{m=-2}^{2} D_{m, 0}^{(2)}\left(\alpha^{C S}, \beta^{C S}, \gamma^{C S}\right) \rho_{2 m}^{C S} \\
& =\sqrt{\frac{3}{2}}\left[\begin{array}{l}
d_{00}^{(2)}\left(\beta^{C S}\right)+\frac{\eta_{C S}}{\sqrt{6}} e^{-2 i \alpha^{C S}} d_{20}^{(2)}\left(\beta^{C S}\right)+ \\
\frac{\eta_{C S}}{\sqrt{6}} e^{2 i \alpha^{C S}} d_{-20}^{(2)}\left(\beta^{C S}\right)
\end{array}\right] \\
& =\sqrt{\frac{3}{2}}\left[\frac{3 \cos ^{2} \beta^{C S}-1}{2}+\frac{\eta_{C S}}{2} \cos 2 \alpha^{C S} \sin ^{2} \beta^{C S}\right]
\end{aligned}
$$

This yields energy eigenvalues given below.

$$
\Delta E_{m \rightarrow m-1}^{C S A}=\hbar \omega_{l}\left(\delta_{i s o, c s}+\delta^{C S}\left[\frac{3 \cos ^{2} \beta^{C S}-1}{2}+\frac{\eta_{C S}}{2} \cos 2 \alpha^{C S} \sin ^{2} \beta^{C S}\right]\right)
$$

For a quadrupolar nucleus, the first-order quadrupolar eigenvalues are derived in a similar fashion, since they are proportional to $A_{20}^{Q}$. The final result for the first-order quadrupolar contribution to the energy splitting is given below in equation 2.62 .

$$
\begin{aligned}
\Delta E_{m \rightarrow m-1}^{(1 Q)} & =\frac{3 e^{2} q Q}{4 I(2 I-1)}(2 m-1)\left[\frac{3 \cos ^{2} \beta^{Q}-1}{2}+\frac{\eta_{Q}}{2} \cos 2 \alpha^{Q} \sin ^{2} \beta^{Q}\right] \\
& =\frac{\hbar 3 C_{Q}}{4 I(2 I-1)}(2 m-1)\left[\frac{3 \cos ^{2} \beta^{Q}-1}{2}+\frac{\eta_{Q}}{2} \cos 2 \alpha^{Q} \sin ^{2} \beta^{Q}\right] \\
C_{Q} & \equiv \frac{e^{2} q Q}{\hbar}
\end{aligned}
$$

Figure 2.3 shows the energy level diagram for a spin $3 / 2$ nucleus for a single orientation of the quadrupolar PAS in the absence of chemical shift anisotropy. As was pointed out 
earlier, for the central transition (where $m=1 / 2$ in the above expression) there is no firstorder quadrupolar contribution to the energy splitting.

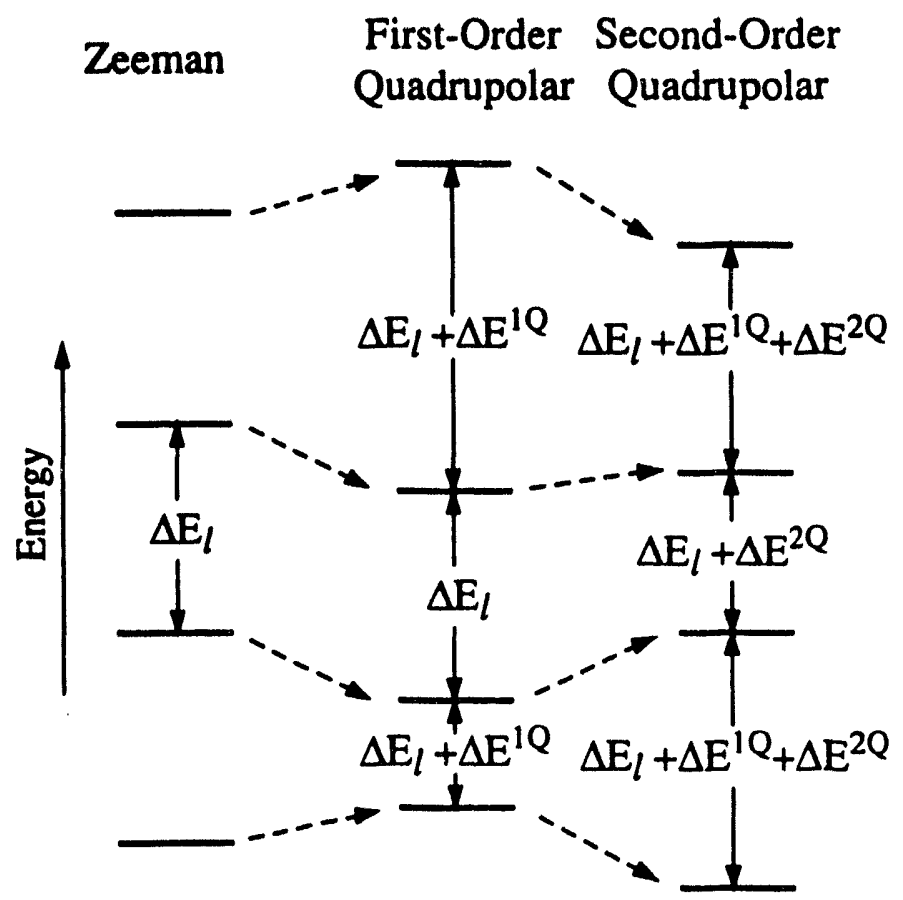

Figure 2.3 Zeeman and Quadrupolar Energy Splitting for $I=3 / 2$ nucleus.

In the case of the central transition, we must also include the second-order quadrupolar correction to the energy splitting as well. This is more difficult to calculate, as the spatial dependence is the sum of two terms. We can first look at the product $A_{21}^{Q} A_{2-1}^{Q}$ by explicitly calculating $A_{21}^{Q}$ and $A_{2-1}^{Q}$.

$$
\begin{aligned}
A_{21}^{Q} & =\sum_{m=-2}^{2} D_{m, 1}^{(2)}\left(\alpha^{Q}, \beta^{Q}, \gamma^{Q}\right) \rho_{2, m}^{Q} \\
& =\sqrt{\frac{3}{2}}\left[\begin{array}{l}
d_{0,1}^{(2)}\left(\beta^{Q}\right) e^{-i \gamma^{Q}}+\frac{\eta_{Q}}{\sqrt{6}} d_{2,1}^{(2)}\left(\beta^{Q}\right) e^{-i \gamma^{Q}-2 i \alpha^{Q}}+ \\
\frac{\eta_{Q}}{\sqrt{6}} d_{-2,1}^{(2)}\left(\beta^{Q}\right) e^{-i \gamma^{Q}+2 i \alpha^{Q}}
\end{array}\right] \\
& =\sqrt{\frac{3}{2} e^{-i \gamma^{Q}}}\left[\begin{array}{l}
\sqrt{\frac{3}{8}} \sin 2 \beta^{Q}+\frac{\eta_{Q}}{\sqrt{6}} i \sin 2 \alpha^{Q} \sin \beta^{Q}- \\
\frac{\eta_{Q}}{\sqrt{6}} \cos 2 \alpha^{Q} \cos \beta^{Q} \sin \beta^{Q}
\end{array}\right]
\end{aligned}
$$




$$
A_{2-1}^{Q}=-\sqrt{\frac{3}{2}} e^{i \gamma^{Q}}\left[\begin{array}{l}
\sqrt{\frac{3}{8}} \sin 2 \beta^{Q}-\frac{\eta_{Q}}{\sqrt{6}} i \sin 2 \alpha^{Q} \sin \beta^{Q} \\
-\frac{\eta_{Q}}{\sqrt{6}} \cos 2 \alpha^{Q} \cos \beta^{Q} \sin \beta^{Q}
\end{array}\right]
$$

Multiplying these together gives $A_{21}^{Q} A_{2-1}^{Q}$.

$$
A_{21}^{Q} A_{2-1}^{Q}=-\frac{1}{16}\left[\begin{array}{l}
9 \sin ^{2} 2 \beta^{Q}+\eta_{Q}^{2} \cos ^{2} 2 \alpha^{Q} \sin ^{2} 2 \beta^{Q}- \\
3 \eta_{Q} \cos 2 \alpha^{Q} \sin ^{2} 2 \beta^{Q}+4 \eta_{Q}^{2} \sin ^{2} 2 \alpha^{Q} \sin ^{2} \beta^{Q}
\end{array}\right]
$$

Likewise, we can calculate the product $A_{22}^{Q} A_{2-2}^{Q}$ from $A_{22}^{Q}$ and $A_{2-2}^{Q}$.

$$
\begin{aligned}
& A_{22}^{Q}=\sqrt{\frac{3}{2}} e^{-2 i \gamma^{Q}}\left[\begin{array}{l}
\sqrt{\frac{3}{8}} \sin ^{2} \beta^{Q}-\frac{\eta_{Q}}{2 \sqrt{6}} i \sin 2 \alpha^{Q} \cos \beta^{Q}+ \\
\frac{\eta_{Q}}{4 \sqrt{6}} \cos 2 \alpha^{Q}\left(1+\cos ^{2} \beta^{Q}\right)
\end{array}\right] \\
& A_{2-2}^{Q}=\sqrt{\frac{3}{2}} e^{2 i \gamma^{Q}}\left[\begin{array}{l}
\sqrt{\frac{3}{8}} \sin ^{2} \beta^{Q}+\frac{\eta_{Q}}{2 \sqrt{6}} i \sin 2 \alpha^{Q} \cos \beta^{Q}+ \\
\frac{\eta_{Q}}{4 \sqrt{6}} \cos 2 \alpha^{Q}\left(1+\cos ^{2} \beta^{Q}\right)
\end{array}\right] \\
& A_{22}^{Q} A_{2-2}^{Q}=\frac{3}{16}\left[\begin{array}{l}
\frac{\eta_{Q}^{2}}{12} \cos ^{2} 2 \alpha^{Q}\left(1+\cos ^{2} \beta^{Q}\right)^{2}+ \\
\eta_{Q} \cos 2 \alpha^{Q}\left(1+\cos ^{2} \beta^{Q}\right) \sin ^{2} \beta^{Q}+ \\
\frac{\eta_{Q}^{2}}{3} \sin ^{2} 2 \alpha^{Q} \cos ^{2} \beta^{Q}+3 \sin ^{4} \beta^{Q}+
\end{array}\right]
\end{aligned}
$$

The sum, $2 A_{21}^{Q} A_{2-1}^{Q}+A_{22}^{Q} A_{2-2}^{Q}$, may be written in terms of products of $\cos 2 i \alpha$ and $\cos 2 j \beta$ where the coefficients $a_{i j}$ are given in table 2.2.

$$
2 A_{21}^{Q} A_{2-1}^{Q}+A_{22}^{Q} A_{2-2}^{Q}=\frac{1}{8} \sum_{i=0}^{2} \sum_{j=0}^{2} a_{i j} \cos 2 i \alpha^{Q} \cos 2 j \beta^{Q}
$$

The energy splitting correction from the second-order quadrupolar effect is then given by equation 2.70 .

$$
\begin{aligned}
\Delta E_{\frac{1}{2} \rightarrow-\frac{1}{2}}^{(2 Q)} & =\frac{\left.\left(e^{2} q Q\right)^{2}(I I I+1)-\frac{3}{4}\right)}{32 I^{2}(2 I-1)^{2} \hbar \omega_{l}} \sum_{i=0}^{2} \sum_{j=0}^{2} a_{i j} \cos 2 i \alpha^{Q} \cos 2 j \beta^{Q} \\
& =\frac{\hbar C_{Q}^{2}\left(I(I+1)-\frac{3}{4}\right)}{32 I^{2}(2 I-1)^{2} \omega_{l}} \sum_{i=0}^{2} \sum_{j=0}^{2} a_{i j} \cos 2 i \alpha^{Q} \cos 2 j \beta^{Q}
\end{aligned}
$$

This energy splitting is shown for a single crystallite orientation in figure 2.3. 


\begin{tabular}{|ccc|ccc|}
\hline$i$ & $j$ & $a_{i j}$ & $i$ & $j$ & $a_{i j}$ \\
\hline 0 & 0 & $-\frac{5}{32}\left(18+\eta_{Q}^{2}\right)$ & 1 & 2 & $-\frac{27}{8} \eta_{Q}$ \\
0 & 1 & $-\frac{9}{4}\left(1-\frac{5}{6} \eta_{Q}^{2}\right)$ & 2 & 0 & $\frac{27}{32} \eta_{Q}^{2}$ \\
0 & 2 & $\frac{9}{32}\left(18+\eta_{Q}^{2}\right)$ & 2 & 1 & $-\frac{9}{8} \eta_{Q}^{2}$ \\
1 & 0 & $\frac{39}{8} \eta_{Q}$ & 2 & 2 & $\frac{9}{32} \eta_{Q}^{2}$ \\
1 & 1 & $-\frac{3}{2} \eta_{Q}$ & & & \\
\hline
\end{tabular}

Table 2.2 Cosine Expansion Coefficients. The $a_{i j}$ coefficients are used in the expansion of the anisotropic portion of the second-order quadrupolar interaction in equation 2.70.

The total energy splitting, including both chemical shift and quadrupolar contributions, under free precession in a high magnetic field may then be given by equation 2.80 .

$$
\Delta E_{m \rightarrow m-1}=\hbar \omega_{l}+\Delta E_{m \rightarrow m-1}^{C S A}+\Delta E_{m \rightarrow m-1}^{(1 Q)}+\Delta E_{m \rightarrow m-1}^{(2 Q)}
$$

It is important to note that in a powder sample, where all orientations of crystallites are present, there exists a continuous distribution of energy splittings corresponding to each individual PAS orientation. This is in contrast to a liquid sample where the rapid motion averages out all orientational dependence of the energy splitting.

\section{Eigenvalues from Rotating Samples}

In the previous section, the expression for the free precession energy splitting was derived. This expression has multiple terms which have anisotropic (orientational) dependences. One of the primary goals of solid state NMR is to acquire spectra which look like liquid-state spectra. The goal of high-resolution isotropic spectra has been approached primarily from two direction. The first is the averaging of anisotropic interaction by averaging the spin operator tensors with multiple-pulse schemes. These techniques work quite well for dipolar interactions (see Haeberlen's book for a more complete review of this subject ${ }^{38}$ ). However, for Hamiltonians such as the truncated chemical shift and quadrupolar interactions, multiple-pulse experiments can only average the anisotropic 
contribution by averaging the isotropic contribution as well (for example, the CPMG pulse sequence $\left.e^{39,40}\right)$. The second and more popular averaging technique involves
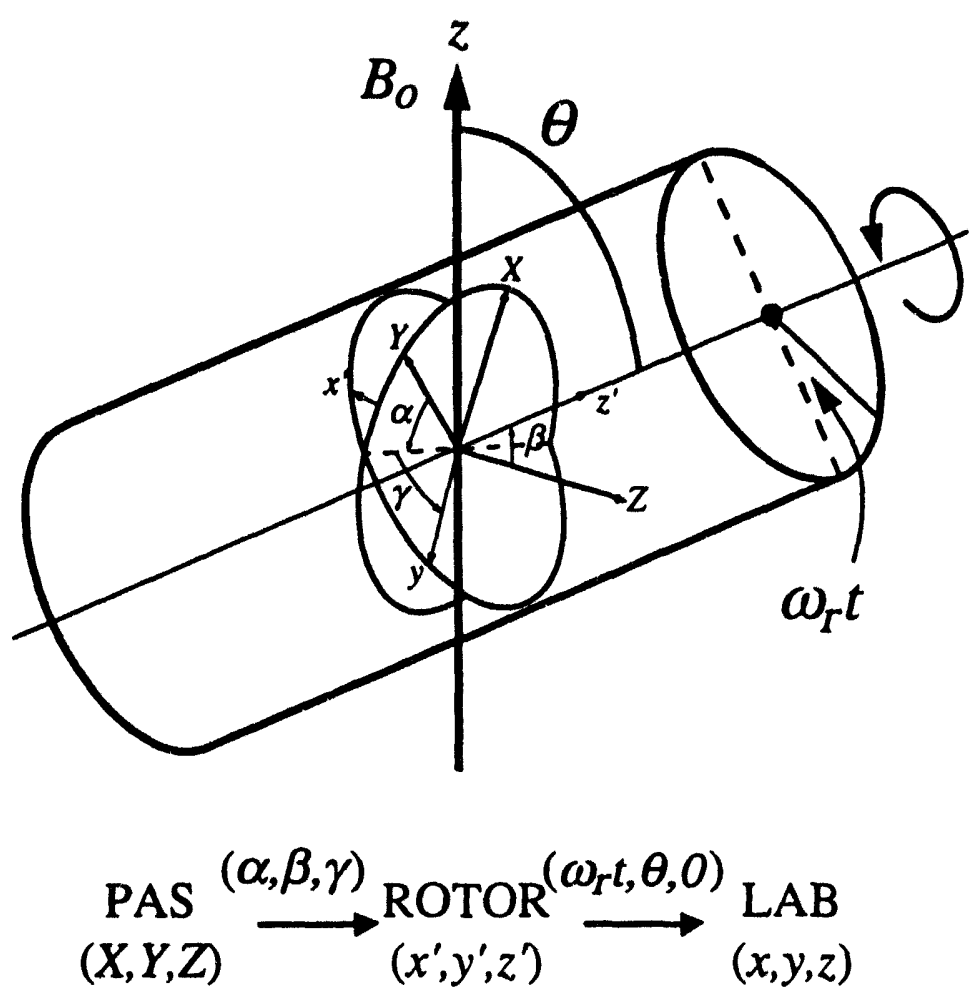

Figure 2.4 PAS to ROTOR to LAB rotations. The Euler angles used in moving from a sample fixed PAS coordinate system to the laboratory system are indicated. This involves multiple rotations.

removing the spatial tensors with time dependent spatial trajectories which approximate isotropic motion. The technique of magic-angle spinning is the most important of these time dependent trajectories. In the magic-angle spinning (MAS) experiment, the sample is rotated rapidly $(>4 \mathrm{kHz})$ about an axis oriented at the angle $\theta_{m}=\cos ^{-1}(1 / \sqrt{3}) \approx 54.74^{\circ}$ with respect to the magnetic field. This has the effect of introducing a time dependence for a general spinning angle to the spatial tensors $A_{l m}^{\lambda}$ given in equation 2.72 .

$$
A_{l m}^{\lambda}=\sum_{n=-l}^{l} \sum_{m^{\prime}=-l}^{l} D_{n m}^{(l)}\left(\omega_{r} t, \theta, 0\right) D_{m^{\prime}, n}^{(l)}\left(\alpha^{\lambda}, \beta^{\lambda}, \gamma^{\lambda}\right) \rho_{l m^{\prime}}^{\lambda}
$$


The $\lambda$ in this expression refers to either the chemical shift, quadrupolar or dipolar interactions. The Euler angles which define the first rotation are $\theta$, the spinning axis angle and $\omega_{r} t$, the time dependent rotation angle. These rotations, along with the usual PAS rotations are shown in figure 2.4 . When rotation is sufficiently rapid (i.e. $\omega_{r}>\omega_{l} \delta^{C S A}$ or $\left.\omega_{r}>\omega_{Q}^{2} / \omega_{l}\right)$, the time dependent $(n \neq 0$ in equation 2.81$)$ terms may be ignored (since they will average to zero) and only the time independent terms remain.

$$
A_{l m}^{\lambda}=d_{0 m}^{(l)}(\theta) \sum_{m^{\prime}=-l}^{l} D_{m^{\prime}, 0}^{(l)}\left(\alpha^{\lambda}, \beta^{\lambda}, \gamma^{\lambda}\right) \rho_{l m^{\prime}}^{\lambda}
$$

For the chemical shift interaction, the important spatial tensor is $A_{20}^{C S}$. In this case, equation 2.82 may be expanded below.

$$
\begin{gathered}
A_{20}^{C S}=\sqrt{\frac{3}{2}}\left(\frac{3 \cos ^{2} \theta-1}{2}\right)\left[\begin{array}{l}
\frac{3 \cos ^{2} \beta^{C S}-1}{2}+ \\
\frac{\eta_{C S}}{2} \cos 2 \alpha^{C S} \sin ^{2} \beta^{C S}
\end{array}\right] \\
\Downarrow \\
\Delta E_{m \rightarrow m-1}^{C S A}=\hbar \omega_{l}\left(\delta_{i s o, c s}+\delta^{C S}\left(\frac{3 \cos ^{2} \theta-1}{2}\right)\left[\begin{array}{l}
\frac{3 \cos ^{2} \beta^{C S}-1}{2}+ \\
\frac{\eta_{C S}}{2} \cos 2 \alpha^{C S} \sin ^{2} \beta^{C S}
\end{array}\right]\right)
\end{gathered}
$$

The only difference between this expression and the previous static expression is the second-order Legendre polynomial $\left(P_{2}[\cos \theta]\right)$ scaling factor. The choice of the magic-angle is now apparent, since this angle is the one which makes the second-order Legendre polynomial zero and eliminates the anisotropic portion of the chemical shift interaction. Likewise, it can be shown that magic-angle spinning can also remove the effects of both homonuclear and heteronuclear dipolar coupling (both of which have a spatial dependence which can be represented as a single second-order tensor). For the quadrupolar interaction, this same analysis holds for the first-order effect when the spinning rate is larger than the quadrupolar coupling constant.

$$
\Delta E_{m \rightarrow m-1}^{(1 Q)}=\frac{3 e^{2} q Q}{4 l(2 I-1)}(2 m-1)\left(\frac{3 \cos ^{2} \theta-1}{2}\right)\left[\frac{3 \cos ^{2} \beta^{Q}-1}{2}+\frac{\eta_{Q}}{2} \cos 2 \alpha^{Q} \sin ^{2} \beta^{Q}\right]
$$


In practice, this may only be observed for nuclei in highly symmetric environment (for example ${ }^{23} \mathrm{Na}$ in $\mathrm{NaCl}$ or ${ }^{79} \mathrm{Br}$ and ${ }^{81} \mathrm{Br}$ in $\mathrm{KBr}$ ) or with a very small nuclear quadrupolar moment (such as the spin $3 / 2$ nuclei ${ }^{7} \mathrm{Li}$ and ${ }^{133} \mathrm{Cs}$ ). Even for these nuclei, spinning sidebands which arise from the time dependent terms are often observed. For quadrupolar nuclei with a large quadrupolar coupling only the central transition is observable (and excitable as well), which has no first-order quadrupolar contribution to the orientation dependence of the energy splitting. The second-order quadrupolar correction does, in fact, show strong orientational dependence. Under fast spinning conditions, just as before, the time dependent contribution to the $A_{21}^{Q} A_{2-1}^{Q}$ and $A_{22}^{Q} A_{2-2}^{Q}$ products may be ignored. As before we may calculate the individual contributions $A_{21}^{Q} A_{2-1}^{Q}$ and $A_{22}^{Q} A_{2-2}^{Q}$.

$$
\begin{aligned}
A_{2 m}^{Q} A_{2-m}^{Q}= & \sum_{j=-2}^{2} \sum_{k=-2}^{2} D_{j, m}^{(2)}\left(\omega_{r} t, \theta, 0\right) D_{k, j}^{(2)}\left(\alpha^{Q}, \beta^{Q}, \gamma^{Q}\right) \rho_{2 k}^{Q} \times \\
& \sum_{n=-2}^{2} \sum_{p=-2}^{2} D_{n, m}^{(2)}\left(\omega_{r} t, \theta, 0\right) D_{p, n}^{(2)}\left(\alpha^{Q}, \beta^{Q}, \gamma^{Q}\right) \rho_{2 p}^{Q}
\end{aligned}
$$

The removal of the time dependent terms is more difficult, since the product must be expanded completely before dropping the time dependent terms. This eliminates all of the terms except those where $j+n=0$. As has been shown earlier by Mueller ${ }^{15}$, the spatial sum may be written as a sum of cosines of the PAS Euler angles $\alpha^{Q}$ and $\beta^{Q}$ in equation 2.77 .

$$
\begin{gathered}
2 A_{21}^{Q} A_{2-1}^{Q}+A_{22}^{Q} A_{2-2}^{Q}=\frac{1}{8} \sum_{i=0}^{2} \sum_{j=0}^{2} a_{i j}^{\prime} \cos 2 i \alpha^{Q} \cos 2 j \beta^{Q} \\
\Downarrow \\
\Delta E_{\frac{1}{2} \rightarrow-\frac{1}{2}}^{(2 Q)}=\frac{\hbar C_{Q}^{2}\left(I(I+1)-\frac{3}{4}\right)}{32 I^{2}(2 l-1)^{2} \omega_{l}} \sum_{i=0}^{2} \sum_{j=0}^{2} a_{i j}^{\prime} \cos 2 i \alpha^{Q} \cos 2 j \beta^{Q}
\end{gathered}
$$

Note that the coefficients $a_{i j}^{\prime}$ are now defined as

$$
a_{i j}^{\prime}=a_{i j}^{(0)}+a_{i j}^{(2)} P_{2}[\cos \theta]+a_{i j}^{(4)} P_{4}[\cos \theta]
$$

where the fourth-order Legendre polynomial $\left(P_{4}[\cos \theta]\right)$ is given in equation 2.88 . 


$$
P_{4}[\cos \theta]=\frac{1}{8}\left(35 \cos ^{4} \theta-30 \cos ^{2} \theta+3\right)
$$

Each component of $a_{i j}^{\prime}$ is defined in table 2.3.

The formulas for the energy eigenvalues under rapid spinning conditions given in equations 2.84 (first-order quadrupolar), 2.86 (second-order quadrupolar) and 2.83 (chemical shift anisotropy) all show both spinning angle and orientational dependence. These dependences manifest themselves in the form of inhomogeneous broadening of the NMR line for powder samples. In the next section, the actual lineshapes resulting from a powder average for the various interactions will be shown. The effect of incomplete averaging due to spinning will be discussed in a later section (see chapter 3 ).

\begin{tabular}{|ccccc|}
\hline$i$ & $j$ & $a_{i j}^{(0)}$ & $a_{i j}^{(2)}$ & $a_{i j}^{(4)}$ \\
\hline 0 & 0 & $-\frac{12}{5}\left(1+\frac{1}{3} \eta_{Q}^{2}\right)$ & $-\frac{12}{7}\left(1-\frac{1}{3} \eta_{Q}^{2}\right)$ & $\frac{81}{1120}\left(18+\eta_{Q}^{2}\right)$ \\
0 & 1 & 0 & $-\frac{36}{7}\left(1-\frac{1}{3} \eta_{Q}^{2}\right)$ & $\frac{9}{56}\left(18+\eta_{Q}^{2}\right)$ \\
0 & 2 & 0 & 0 & $\frac{9}{32}\left(18+\eta_{Q}^{2}\right)$ \\
1 & 0 & 0 & $\frac{24}{7} \eta_{Q}$ & $\frac{81}{56} \eta_{Q}$ \\
1 & 1 & 0 & $-\frac{24}{7} \eta_{Q}$ & $\frac{27}{14} \eta_{Q}$ \\
1 & 2 & 0 & 0 & $-\frac{27}{8} \eta_{Q}$ \\
2 & 0 & 0 & 0 & $\frac{27}{32} \eta_{Q}^{2}$ \\
2 & 1 & 0 & 0 & $-\frac{9}{8} \eta_{Q}^{2}$ \\
2 & 2 & 0 & 0 & $\frac{9}{32} \eta_{Q}^{2}$ \\
\hline
\end{tabular}

Table 2.3 Coefficients in Anisotropic Cosine Expansion for the 2nd-Order Quadrupolar Correction Under Fast Spinning Conditions.

\section{Lineshape simulations}

In the absence of spinning, the static eigenvalue equations may be used to obtain the theoretical spectra observed from both spin $1 / 2$ and quadrupolar nuclei. In all cases where a powder sample is simulated the assumptions are the same. First, all orientations 
of the crystallite PAS are equally probable (there is no sample alignment or preferential geometry of the crystallites in the powdered sample). Second, all orientations of the crystallite PAS are present in the sample. Third, the contribution from each crystallite to the spectrum will be equal. With these three rules, we can construct a powder averaged spectrum for a static or rotating sample. Mathematically, the process of calculating the intensity at a given frequency (or energy) is equivalent to doing the following integration over the sample.

$$
I(\omega)=\int_{0}^{2 \pi} \int_{0}^{\pi} \delta(\hbar \omega-\Delta E(\alpha, \beta)) \sin \beta d \beta d \alpha
$$

In this expression, the $\sin \beta$ scaling factor is included when converting an integral over a unit sphere in Cartesian coordinates to spherical coordinates, to maintain a constant $d \beta d \alpha$ solid angle. Also, the Dirac delta function is defined below.

$$
\delta(a-b)=\left\{\begin{array}{l}
0 \rightarrow a \neq b \\
1 \rightarrow a=b
\end{array}\right.
$$

This formula may then be used to create a spectrum by performing the integrals for each frequency within the sweepwidth of the spectrum. (We will also use equation 2.90 later in chapter 3 when calculating spinning sideband positions and intensities.) Spectra simulated in this fashion are shown in figure 2.5 for static spin $1 / 2$ nuclei and quadrupolar nuclei.

It may be seen that the central transition goes off scale in the first-order quadrupolar spectrum in figure 2.5. This is because, in the absence of chemical shift anisotropy or dipolar broadening, the central transition has no first-order broadening. In the second order quadrupolar spectrum, none of the satellite (outer) transitions are shown, as they are of negligible intensity in the sweepwidth shown. 
This method of simulating spectra works equally well for spinning samples. In figure 2.6, the high speed magic-angle spinning spectra of samples with identical parame-

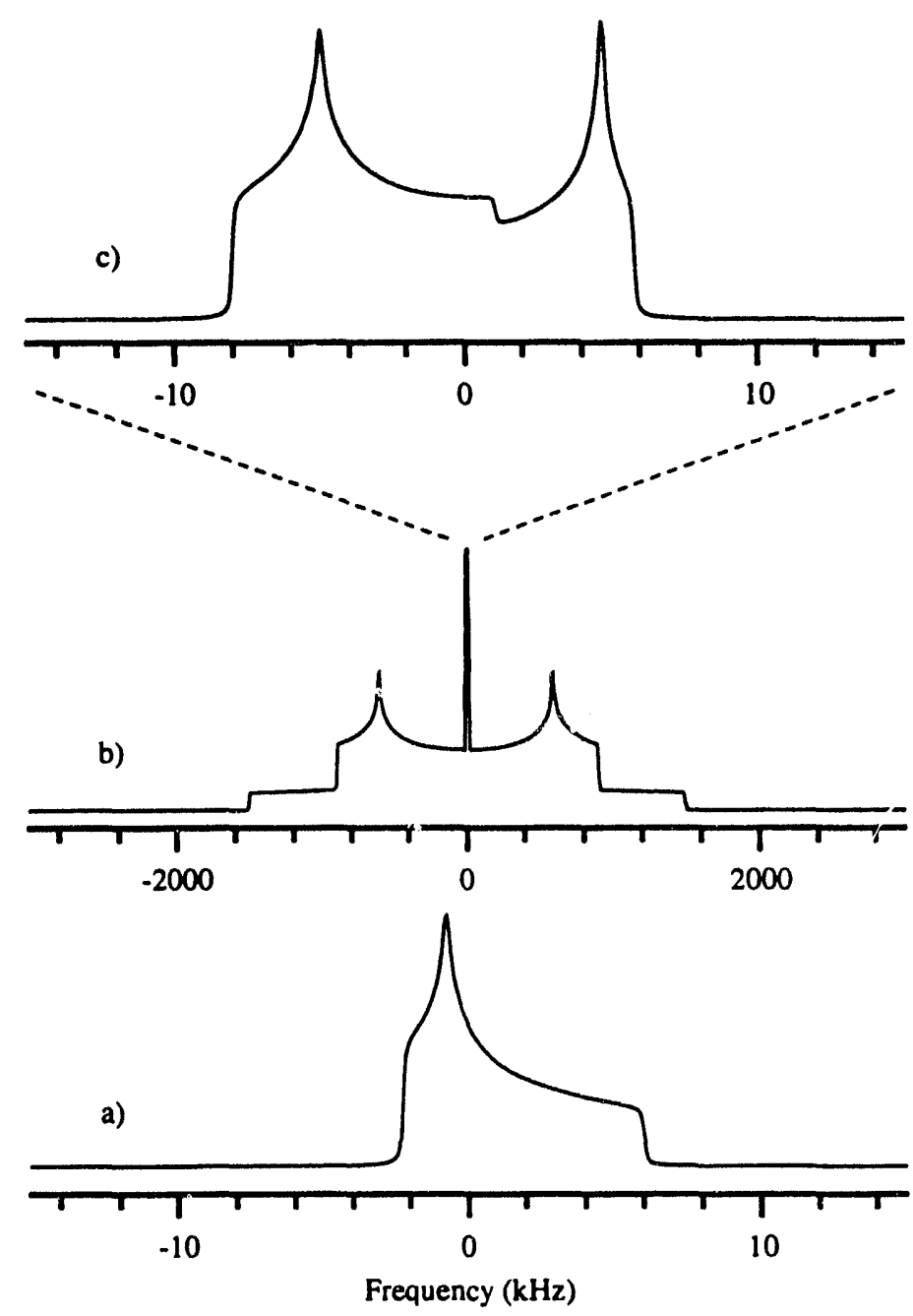

Figure 2.5 Static CSA $(a), 1$ st Order (b) and 2nd Order (c) Quadrupolar Lineshapes. For the chemical shift anisotropy simulation, $\omega_{l}$ was $100 \mathrm{MHz}, \delta_{i s o, c s}$ was $10 \mathrm{ppm}, \delta C S$ was $50 \mathrm{ppm}$ and $\eta_{C S}$ was 0.3. For the 1st Order and 2nd Order Quadrupolar lineshapes the $\omega_{l}$ was $100 \mathrm{MHz}, \delta_{\text {iso,cs }}$ was $10 \mathrm{ppm}, I$ was $3 / 2, \omega_{Q}$ was $3.0 \mathrm{MHz}, \eta_{Q}$ was 0.2 .

ters as in figure 2.5 are simulated. The spectrum of the spin $1 / 2$ nucleus is completely averaged into a single narrow line under MAS. This is expected, as all of the anisotropic contribution to the energy splitting is averaged to zero. The second-order quadrupolar MAS spectrum, however, is still quite complex and only about a factor of 3 narrower than the static spectrum. This is expected as well since the second and fourth-order Legendre 
polynomials never simultaneously approach zero. In fact, this demonstrates the basic
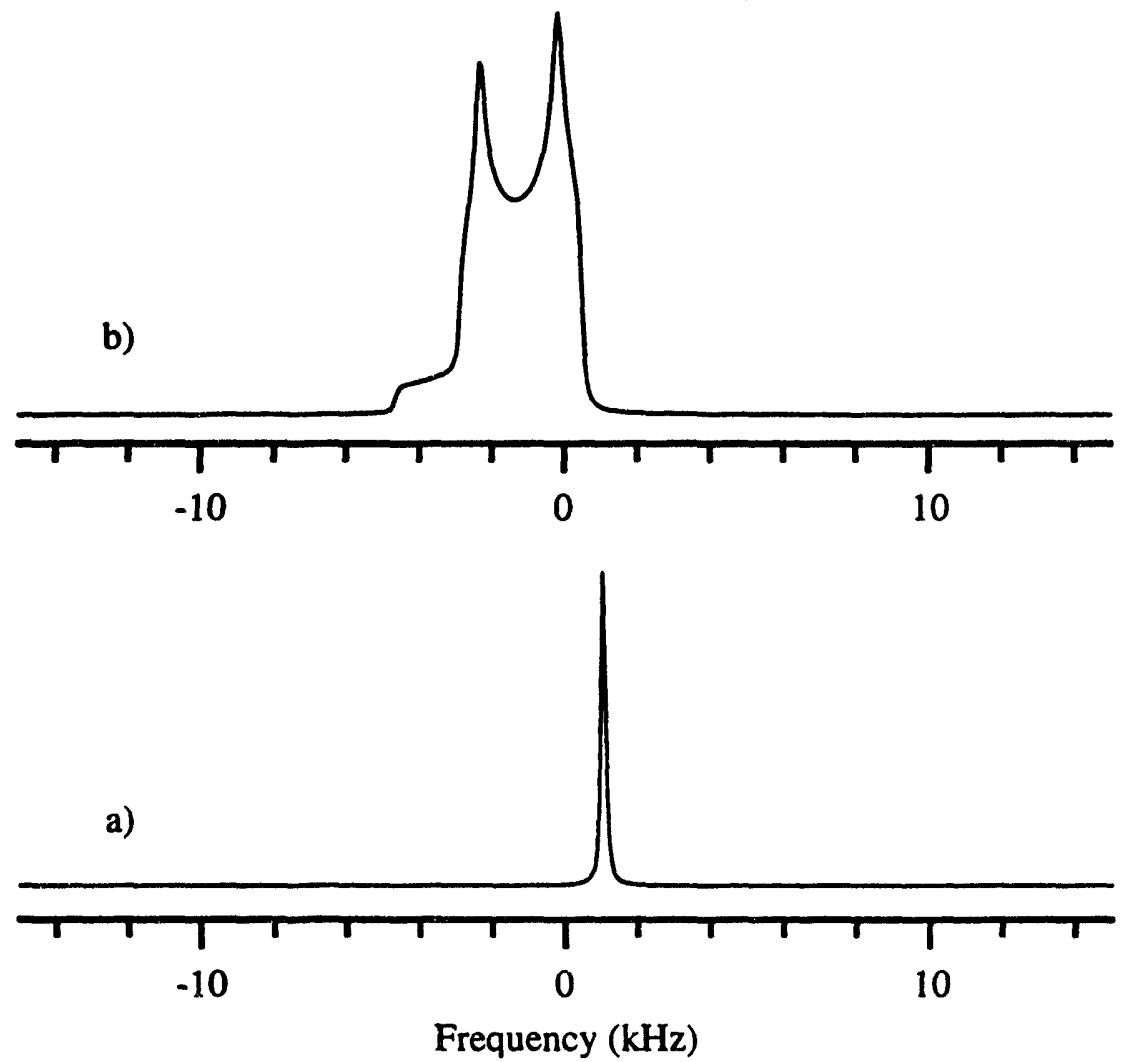

Figure 2.6 MAS CSA (a) and 2nd Order (b) Quadrupolar Lineshapes. The simulation parameters are identical to those in figure 2.6. Notice that while the chemical shift anisotropy is completely averaged by MAS, the 2 nd order quadrupolar interaction is only slightly reduced (about a factor of 3 ) from the static simulation.

problem in observing the central transition of quadrupolar nuclei. There is no single spinning axis which completely removes all of the anisotropic broadening to yield a liquid-like spectrum (analogous to MAS for spin 1/2 nuclei). In figure 2.7, variable-angle spinning (VAS) spectra are shown for a variety of spinning angles and quadrupolar asymmetry parameters $\eta_{Q}$ showing the range of both shoulder and singularity locations. 
A problem with the previously mentioned simulation scheme is the speed at which spectra can be simulited. If we wish to use a least squares minimization approach to simulating chemical shift and quadrupolar broadened spectra, the aforementioned method is too slow. A slightly faster method is to divide the overall sweep width into $N$

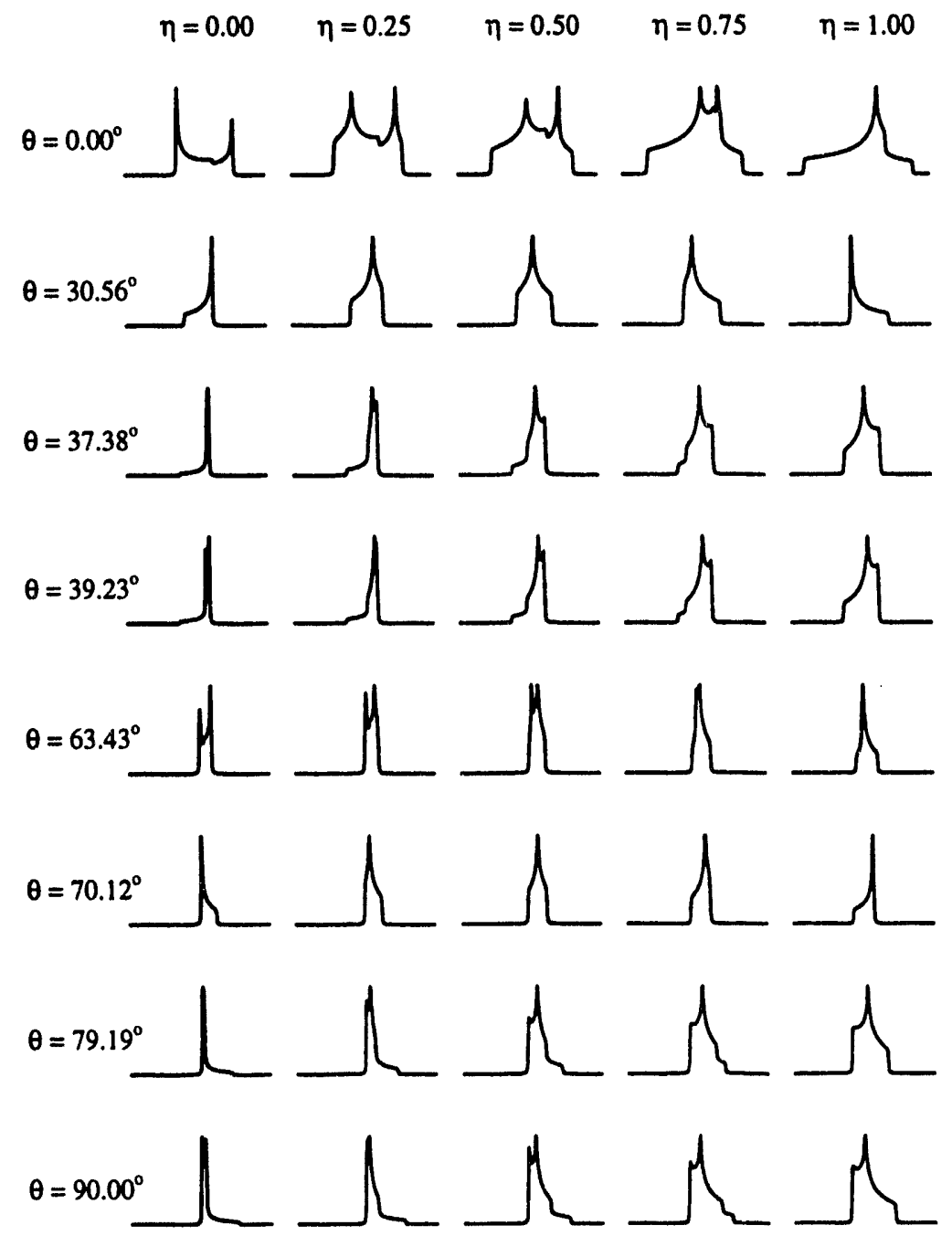

Figure 2.7 Quadrupolar VAS Spectra. All spectra are simulated assuming fast spinning limit and constant $C_{Q}$ and variable angle $\theta$ and $\eta_{Q}$.

bins. The angles $\alpha$ and $\beta$ are then looped over their integration limits in small step sizes and the frequency at each point is calculated. The scaling factor, $\sin \beta$, intensity is then added to the appropriate frequency bin for this point. In this method, a large number of steps (often over 200 for each angle) must be taken for both $\alpha$ and $\beta$ to obtain spectra 
with correct intensities across the spectrum. Using interpolation to divide the intensity between bins for frequencies which fall between two bin positions fails to give significantly better results (this type of interpolation can reduce the number of steps by perhaps factor of 2).

A second method developed by Alderman et al. ${ }^{41}$ is a much more efficient scheme for calculating powder spectra. In this approach, the surface of a unit sphere (over which integration is performed) is collapsed onto an enclosed octahedron. The advantage of an octahedron is that the face may be divided into a large number of triangles (rather than curved rectangles on a sphere). The first advantage is that it is much easier to interpolate over three points (arising from the division of the surface into triangles) than four (which occurs when the two euler angles $\alpha$ and $\beta$ are stepped in regular steps as in the first method). The second advantage is that the surface may be parameterized into three rational numbers (by dividing each edge of the octahedron into some integer number of segments and counting with integers along each edge) representing the $x, y$ and $z$ coordinates. The formula for these coordinates are linear rather than quadratic in the case of a sphere. The third advantage is that the calculation of sines and cosines is simplified, as it merely ratios of surface coordinates rather than actual calculation of trigonometric functions. Computer programs which utilize these algorithms are discussed and shown in the appendix. 


\section{Chapter 3}

\section{Dynamic-angle spinning DAS}

In the previous chapter, the orientational dependences of the chemical shift interaction and the second-order quadrupolar interaction were derived. It was shown that there exists no single spinning angle which averages the second-order quadrupolar interaction completely. Dynamic-angle spinning (DAS) was developed simultaneously by both Pines et al. ${ }^{42}$ and Virlet $e t$ al. ${ }^{43}$ This technique is useful for obtaining high resolution isotropic spectra of quadrupolar nuclei in powdered samples. Specifically, DAS does average both the first-order chemical shift anisotropy and the second-order quadrupolar interaction. Previously, this technique has been used to study ${ }^{11} \mathrm{~B}^{44},{ }^{17} \mathrm{O}^{45}$ ${ }^{48},{ }^{23} \mathrm{Na}^{42,49.50},{ }^{27} \mathrm{Al}^{51}$ and ${ }^{87} \mathrm{Rb}^{49.52-54}$ in a variety of compounds. In most of these cases, the technique of DAS provides orders of magnitude improvement in overall resolution in the isotropic dimension over MAS or static experiments. In the next section, I will review some of the theory and history of the DAS experiment.

\section{History of DAS}

The roots of dynamic-angle spinning lie in the frequency formula for a second order quadrupolar interaction. It can be seen (after recombining terms in equation 2.86) that there are three terms for a crystallite of an arbitrary orientation spinning rapidly about an axis oriented at $\theta$ with respect to the magnetic field.

$$
\begin{aligned}
\omega^{(2 Q)}\left(\alpha^{Q}, \beta^{Q}, \theta\right) & =\omega_{i s o}^{(2 Q)}+A_{2}\left(\alpha^{Q}, \beta^{Q}\right) P_{2}[\cos \theta]+A_{4}\left(\alpha^{Q}, \beta^{Q}\right) P_{4}[\cos \theta] \\
\omega_{i s o}^{(2 Q)} & =\frac{-3\left(I(I+1)-\frac{3}{4}\right)}{40 \omega_{l} I^{2}(2 l-1)^{2}} C_{Q}^{2}\left(1+\frac{\eta_{Q}^{2}}{3}\right)
\end{aligned}
$$

The first term represents the second-order quadrupolar isotropic shift. The other two, $A_{2}$ and $A_{4}$, represent the orientationally dependent coefficients of the second and fourth-order Legendre polynomials. Figure 3.1 shows both the second and fourth-order Legendre 
polynomials. It is immediately apparent that the fourth order roots $\left(30.56^{\circ}\right.$ and $\left.70.12^{\circ}\right)$ do not correspond to the second order root $\left(54.74^{\circ}\right)$. This demonstrates the futility of spinning about a single axis to achieve high resolution quadrupolar spectra.

The solution is to use two different spinning angles in the averaging of the quadrupolar interaction. Dynamic-angle spinning is just one of these solutions.

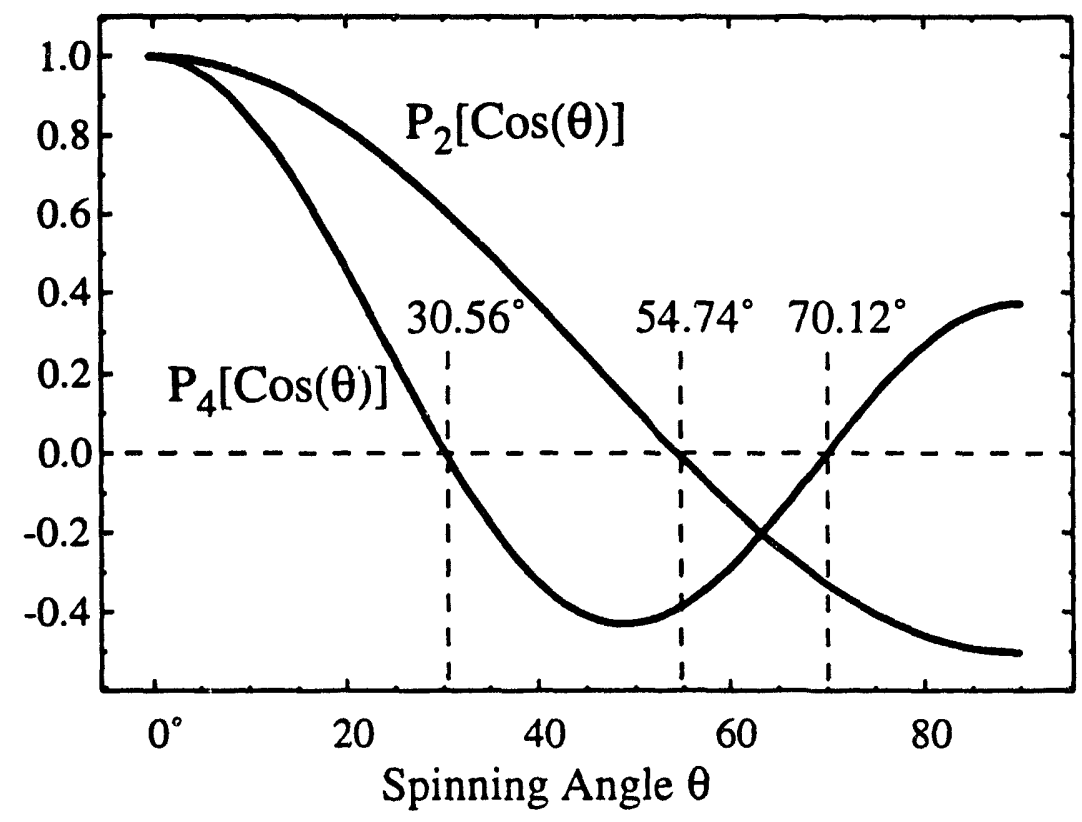

Figure 3.1 2nd and 4th Order Legendre Polynomials. There is no single angle at which both the 2 nd and 4 th order polynomials are zero. Therefore, multiple angles will be needed to average the second-order quadrupolar interaction (equation. 3.1).

Alternative solutions such as dynamic-angle hopping and double rotation will be discussed in chapter 6 of this thesis. ${ }^{55,56}$ In this experiment, the sample is allowed to undergo free precession following a $90^{\circ}$ pulse at a first angle $\theta_{1}$ for a time $t_{1} /(k+1)$. A zfilter is used to store the magnetization during a hopping period, in which the rotor spinning axis is changed from $\theta_{1}$ to $\theta_{2}$. At a time $k t_{1} /(k+1)$ following the second pulse of the $\mathrm{z}$-filter, a dynamic-angle spinning echo will appear. This is shown below schematically in figure 3.2. The evolution of the density matrix will be the product of two unitary 
operators given by the evolution at each angle. The unitary evolution operators are shown below in equation 3.2 .

$$
\begin{aligned}
& U\left(\theta_{1}\right)=\exp \left[-i \omega^{(2 Q)}\left(\alpha^{Q}, \beta^{Q}, \theta_{1}\right) t_{1} I_{z} /(k+1)\right] \\
& U\left(\theta_{2}\right)=\exp \left[\begin{array}{l}
\left(-i \omega^{(2 Q)}\left(\alpha^{Q}, \beta^{Q}, \theta_{2}\right) k t_{1} I_{z} /(k+1)\right)+ \\
\left(-i \omega^{(2 Q)}\left(\alpha^{Q}, \beta^{Q}, \theta_{2}\right) t_{2} I_{2}\right)
\end{array}\right] \\
& \rho\left(t_{1}, t_{2}\right)=U\left(\theta_{2}\right) U\left(\theta_{1}\right) \rho(0) U^{\dagger}\left(\theta_{1}\right) U^{\dagger}\left(\theta_{2}\right)
\end{aligned}
$$

The assumption made in equation 3.2 is that the $\mathrm{z}$-filter does not change the density matrix at all. In any single scan, this of course is impossible, however by proper choice of the phase cycle, the density matrix can be reconstructed over multiple scans so that this equation is true. The coherence pathway needed to accomplish this is shown in
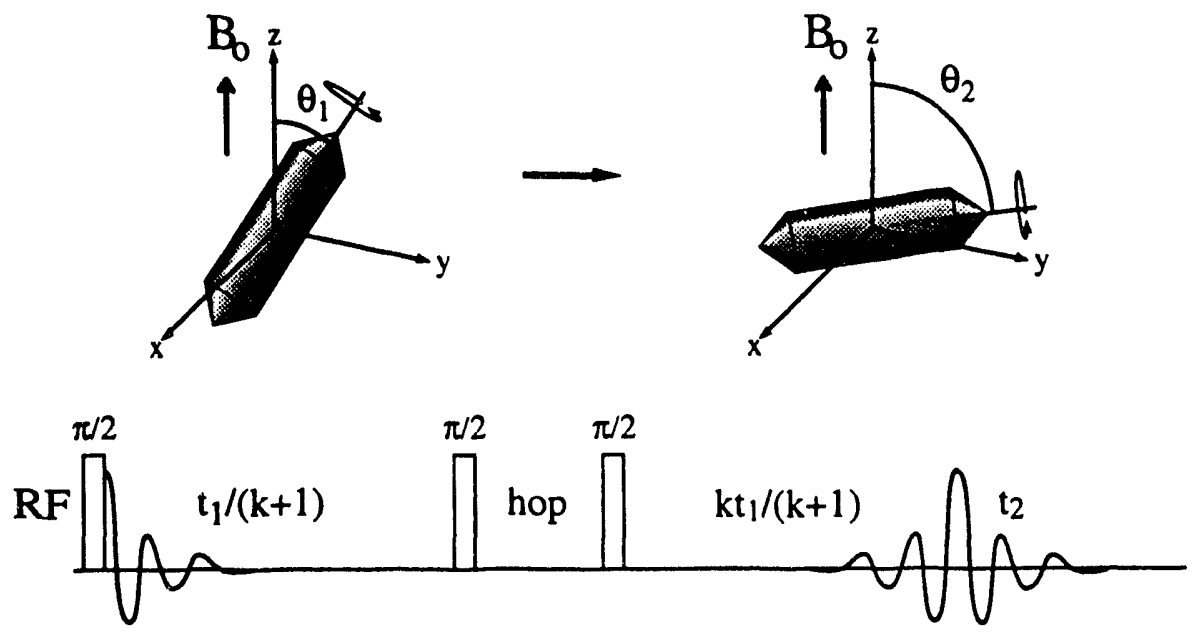

Figure 3.2 DAS Experiment and Pulse Sequence. In this experiment, the value of $t_{1}$ is incremented in a two dimensional fashion. The $t_{1}$ dimension signal gives the isotropic DAS spectrum while the second dimension contains information about the anisotropy of both the chemical shift and quadrupolar interactions.

figure 3.3. Note that the coherence is -1 both before and after the $z$-filter, indicating that the density matrix is unaffected by the z-filter (except for relaxation which merely scales the size of the density matrix uniformly). The minimum phase cycle needed to select this coherence pathway (assuming good receiver quadrature) is given below. 


$$
\left(\phi_{1}, \phi_{2}, \phi_{3}, \phi_{r}\right) \Rightarrow\left\{\begin{array}{l}
(0,0,0,0),(90,0,0,90), \\
(180,0,0,180),(270,0,0,270), \\
(180,180,0,0),(270,180,0,90), \\
(0,180,0,180),(90,180,0,270)
\end{array}\right\}
$$

This cycle is arrived at by noticing first that we need to guarantee a -1 coherence after the first pulse and therefore cycle this pulse through 4 independent phases. The second pulse is cycled through 2 independent phases, giving either a $\Delta p$ of +1 or -1 . Only the +1

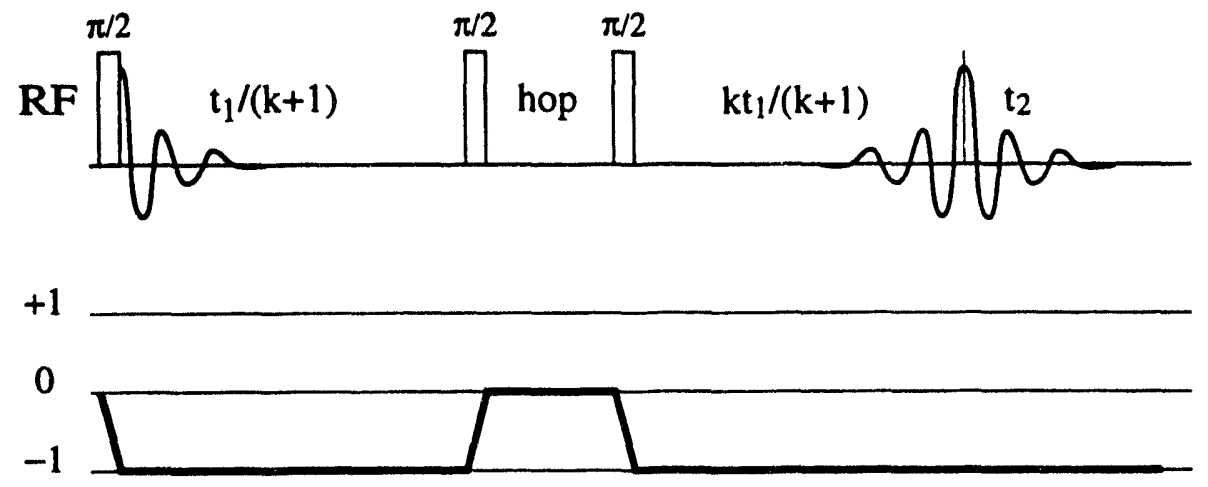

Figure 3.3 DAS Pulse Sequence Coherence Pathway. The initial -1 pathway may be selected by phase cycling the first pulse through 4 independent phases. The $+1 \Delta p$ at the second pulse may be achieved by cycling through 2 independent phases (since the $-1 \Delta p$ would produce a net -2 coherence, which cannot be present in this system). This indicates that a complete phase cycle of 8 is needed to get artifact free spectra (since the quadrature of the receiver selects the final $-1 \Delta p$ ).

coherence transfer is meaningful and puts the coherence at 0 , which is equivalent to Zeeman order. This coherence will relax with rate $T_{1}$ during the rotor axis reorientation period, after which an uncycled $90^{\circ}$ pulse is used to bring the coherence to the -1 level again (the +1 and 0 coherences will be unobservable with our receiver). The equation which describes the relationship between the phases is given below.

$$
-\phi_{1}+\phi_{2}-\phi_{3}+\phi_{r}=0
$$

The observed signal may then be calculated, knowing that the initial density matrix is $\rho(0)=I_{-1}$ following the first $90^{\circ}$ pulse. 


$$
\begin{aligned}
S\left(t_{1}, t_{2}\right) & =\operatorname{tr}\left\{\rho\left(t_{1}, t_{2}\right) I_{+1}\right\} \\
& =\exp \left[\begin{array}{l}
-i\left(\begin{array}{l}
\omega^{(2 Q)}\left(\alpha^{Q}, \beta^{Q}, \theta_{1}\right) t_{1}+ \\
k \omega^{(2 Q)}\left(\alpha^{Q}, \beta^{Q}, \theta_{2}\right) t_{1}
\end{array}\right) /(k+1)
\end{array}\right] \times \\
& \exp \left[-i \omega^{(2 Q)}\left(\alpha^{Q}, \beta^{Q}, \theta_{2}\right) t_{2}\right]
\end{aligned}
$$

The key to the entire DAS experiment may be seen clearly in equation 3.5. If the $t_{1}$-dependent part can be made to be purely isotropic through proper choice of $\theta_{1}$ and $\theta_{2}$, then the entire problem is solved. To do this we set the $t_{1}$-dependent sum of two terms in this exponential equal to $(k+1) \omega_{i s o}^{(2 Q)} t_{1}$ for all values of both orientation and time.

$$
\begin{gathered}
(k+1) \omega_{i s o}^{(2 Q)} t_{1}=\omega^{(2 Q)}\left(\alpha^{Q}, \beta^{Q}, \theta_{1}\right) t_{1}+k \omega^{(2 Q)}\left(\alpha^{Q}, \beta^{Q}, \theta_{2}\right) t_{1} \\
\Downarrow \\
0=A_{2}\left(\alpha^{Q}, \beta^{Q}\right) P_{2}(\cos \theta)+A_{4}\left(\alpha^{Q}, \beta^{Q}\right) P_{4}(\cos \theta)+ \\
A_{2}\left(\alpha^{Q}, \beta^{Q}\right) k P_{2}(\cos \theta)+A_{4}\left(\alpha^{Q}, \beta^{Q}\right) k P_{4}(\cos \theta)
\end{gathered}
$$

In this final expression, we know that the Legendre polynomials will not both simultaneously be zero (from figure 3.1). Also, the orientationally dependent coefficients will likewise be non-zero for most orientations. The only absolute soiution is for the following pair of equations to be true.

$$
\begin{aligned}
& P_{2}\left(\cos \theta_{1}\right)=-k P_{2}\left(\cos \theta_{2}\right) \\
& P_{4}\left(\cos \theta_{1}\right)=-k P_{4}\left(\cos \theta_{2}\right)
\end{aligned}
$$

This guarantees that for all orientations, the anisotropic terms will cancel in the $t_{1}$ evolution, leaving a purely isotropic evolution.

$$
S\left(t_{1}, t_{2}\right)=\exp \left[-i \omega_{i s o}^{(2 Q)} t_{1}\right] \exp \left[-i \omega^{(2 Q)}\left(\alpha^{Q}, \beta^{Q}, \theta_{2}\right) t_{2}\right]
$$

Equation 3.7 is a system of two equations with three unknowns. This means that there will be a continuous distribution of solutions which may be parameterized by $k$. These angles are shown below in figure 3.4. The usual pair of angles used for DAS experiments are the $k=1$ pair $\left(37.38^{\circ}\right.$ and $\left.79.19^{\circ}\right)$ and the $k=5$ pair $\left(0.00^{\circ}\right.$ and $\left.63.43^{\circ}\right)$. The reasons 
for the choice of $k=5$ or $k=1$ will be discussed later, however, any other angle pair meeting the criterion of equation 3.7 will work as well. The solutions (as a function of $k$ )

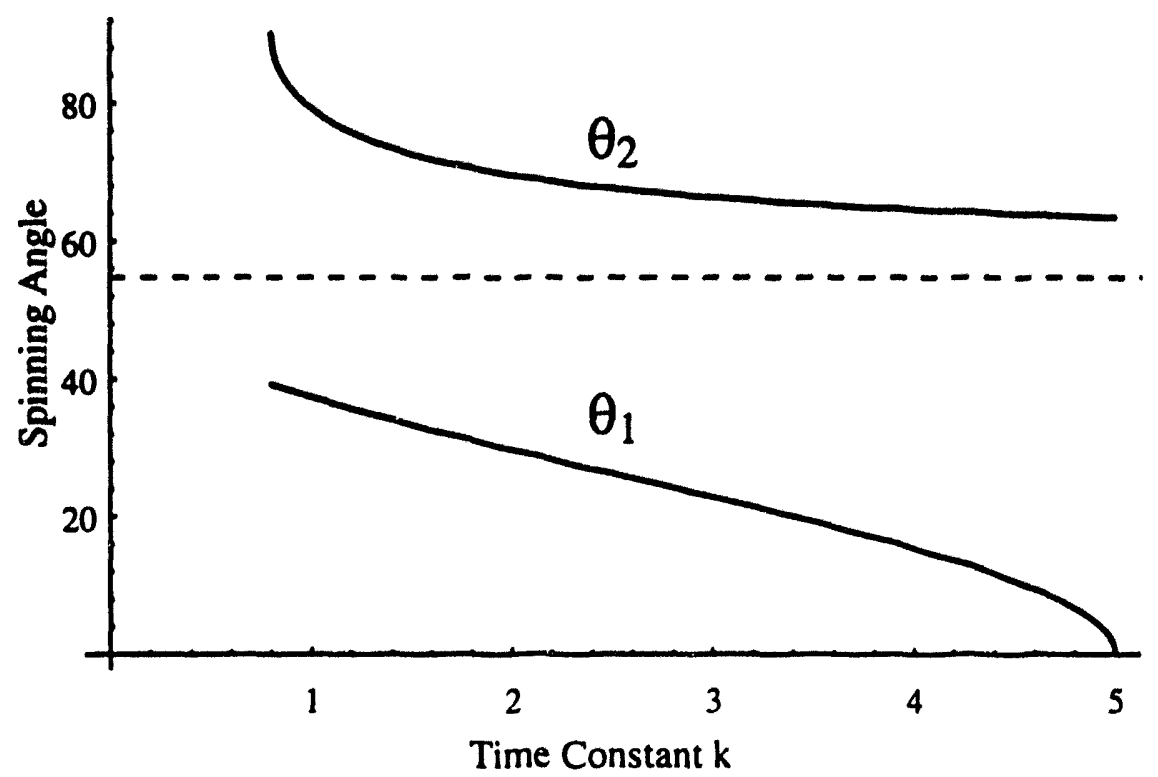

Figure 3.4 DAS Angle Pairs. The angles $\theta_{1}$ and $\theta_{2}$ are solutions to equation 3.6 as parameterized by $k$ given in equation 3.9. It is interesting to note that the magic-angle (shown with a dotted line) is not included in the possible solutions to the DAS equations.

to the pair of equations 3.7 are given below and were used to generate the curves in figure 3.4 .

$$
\begin{aligned}
& \cos \theta_{1}=\sqrt{\frac{1+2 \sqrt{\frac{k}{5}}}{3}} \\
& \cos \theta_{2}=\sqrt{\frac{1-2 \sqrt{\frac{1}{5 k}}}{3}}
\end{aligned}
$$

One of the first samples for which a DAS spectrum was collected was from the ${ }^{23} \mathrm{Na}$ nucleus in sodium oxalate $\left(\mathrm{Na}_{2} \mathrm{C}_{2} \mathrm{O}_{4}\right) .{ }^{42}$ The spectrum in figure 3.5 represents the Fourier transform of the DAS echo tops which corresponds to the signal at $t_{2}=0$. This spectrum was taken at a magnetic field strength of $11.7 \mathrm{~T}\left(132.7 \mathrm{MHz}\right.$ for ${ }^{23 \mathrm{Na}}$ ) with a homebuilt DAS probe which has was designed by Mueller et al. ${ }^{15,51}$ The angle pair for this experiment is the $k=1$ set of $37.38^{\circ}$ and $79.19^{\circ}$. This represents a total of 512 scans 
for each of the $128 t_{1}$ points which have been zero filled to 512 points before Fourier transforming. The ${ }^{23} \mathrm{Na} \pi / 2$ central transition selective pulses were $5.0 \mu$ s while the

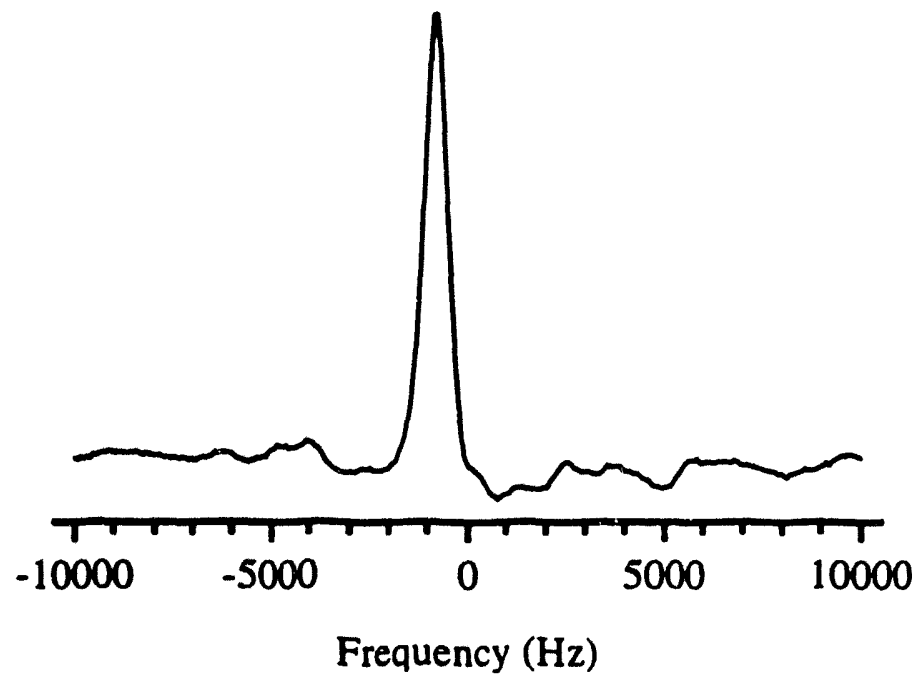

Figure 3.5 Example 1D DAS Spectrum of Sodium Oxalate. This spectrum was acquired at $11.7 \mathrm{~T}$ by Fourier transforming the DAS echo tops taken at $t_{2}=0$.

hopping time was $30.0 \mathrm{~ms}$. The magic-angle was set using the usual method of maximizing the number of ${ }^{81} \mathrm{Br}$ spinning sidebands present in an internal $\mathrm{KBr}$ angle standard ${ }^{2} \mathrm{H}$ in deuterated HMB or DMB will also work equally well) as has been discussed previously. ${ }^{15}$ The overall linewidth of the isotropic site is about $700 \mathrm{~Hz}$. This is significantly narrower than the approximately $3-4 \mathrm{kHz}$ wide line seen in variable-angle spectra of sodium oxalate. The theory for the limiting linewidth of DAS peaks such as this will be described in the last section of this chapter.

Dynamic-angle spinning data may alternatively be processed by Fourier transforming with respect to both dimensions. The resulting two-dimensional DAS spectrum has phase twist lineshape (see chapter 4) and to make the data presentable, it is viewed in magnitude mode (where this operation is performed by calculating the magnitude of each complex point in the spectrum). The 2D DAS spectrum is shown for sodium oxalate in figure 3.6. This experiment has 128 points in $t_{1}$ (the isotropic DAS dimension) and 128 points in $t_{2}$ (the anisotropic VAS dimension). Other parameters are identical to the previ- 
ous spectrum. The spectral width in each dimension is indicated on the plot. The projections onto both axes are shown on top and to the right of the contour plot. Notice that

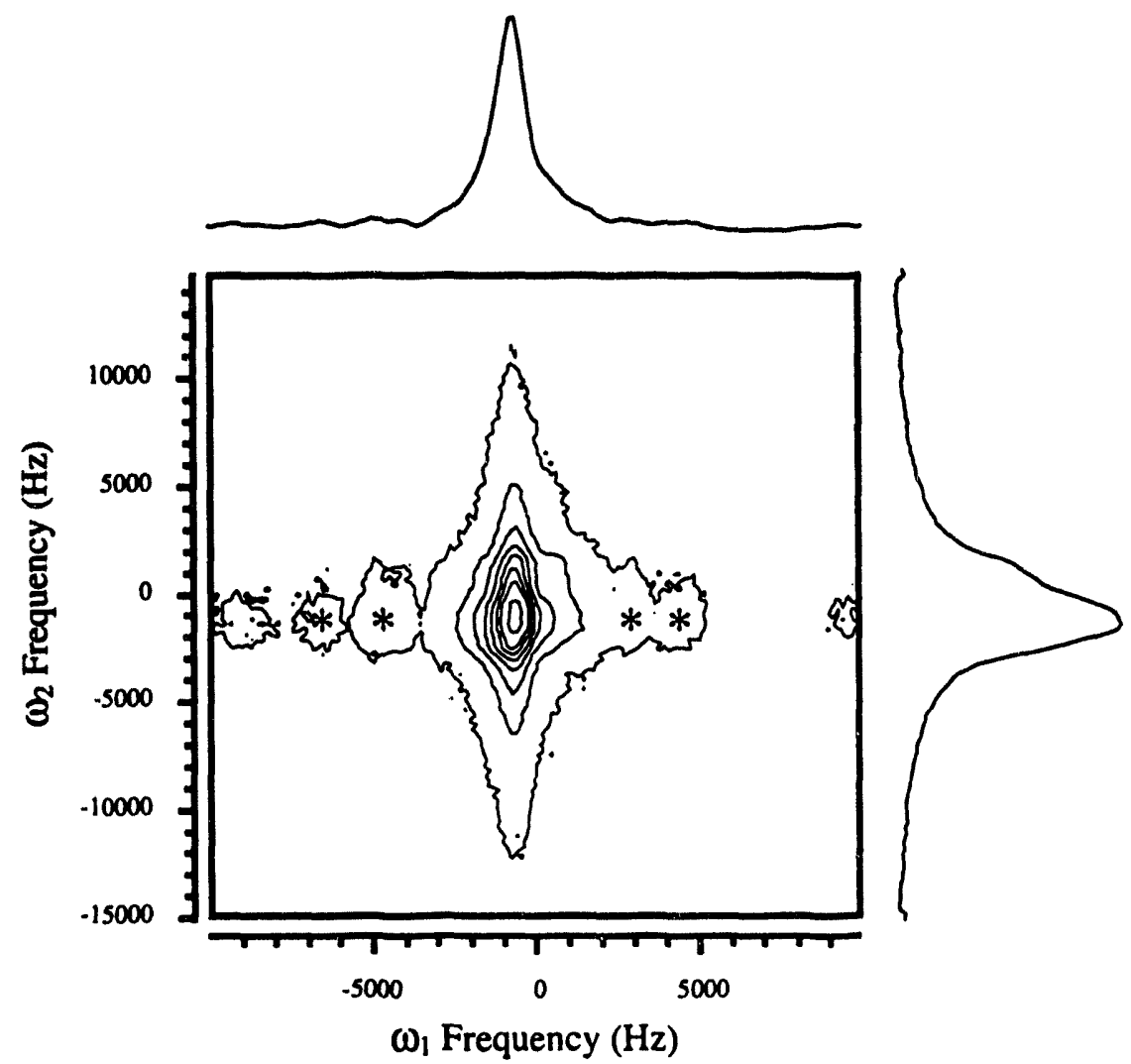

Figure 3.6 Example 2D DAS Spectrum of Sodium Oxalate. This spectrum was acquired at $11.7 \mathrm{~T}$ by performing a 2D Fourier transform of the DAS data set. The data is presented in magnitude mode to avoid the phase twist lineshapes. Asterisks indicate spinning sidebands.

two spinning sidebands on either side of the isotropic peak are indicated with asterisks.

The theory describing both their intensity and position will be presented in the next section. Also, it is apparent that the presentation of the data in magnitude mode leads to much broader lines than the absorption mode 1D spectrum seen in figure 3.5 (compare to the isotropic projection onto the $\omega_{1}$ dimension in figure 3.6). A method for acquiring pure-absorption phase spectra in two dimensions will be discussed in chapter 4 . These spectra demonstrate the potential of DAS to successfully average second-order quadrupolar interactions. 


\section{Spinning Sidebands}

The appearance of spinning sidebands in the DAS spectra shown in figure 3.7 leads us directly into a discussion of their location and intensity in NMR experiments. Shown below in figure 3.7 are the slow spinning MAS simulations of both a spin $1 / 2$ and spin $3 / 2$ nucleus. The simulation parameters are identical to figures 2.5 and 2.6 , with the

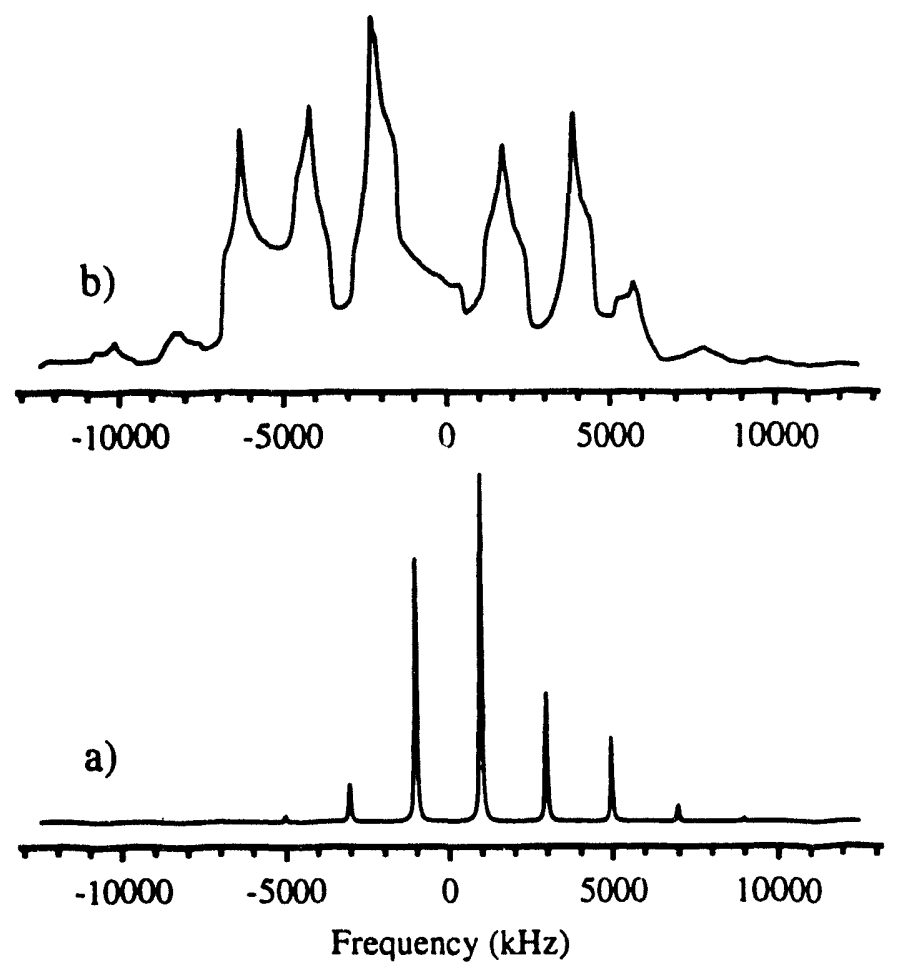

Figure 3.7 Sidebands in MAS Spectra of CSA and Second-Order Quadrupolar Broadened Sites. Simulation parameters were identical to figure 2.5 and $2.6\left(\delta_{i s o, c s}=10 \mathrm{ppm}, \delta C S=\right.$ $50 \mathrm{ppm}, \eta_{C S}=0.3$ and $C_{Q}=3.0 \mathrm{MHz}, \eta_{Q}=0.2$ ) with the added parameter of a spinning speed of $2.0 \mathrm{kHz}$.

spinning speed given as $2.0 \mathrm{kHz}$. It is immediatly noticeable that slow spinning produces additional lines not observed in the spectra in figures 2.5 and 2.6. In the case of a spin $1 / 2$ nucleus, the additional spinning sidebands do not significantly hinder interpretation of the spectrum. The only major difficulty in this case comes in integration and identification of the isotropic chemical shift. The integration problem is overcome by adding together the integrated intensity from families of spinning sidebands in the case of multiple sites. ${ }^{57}$ The problem of identifying isotropic sites may be overcome by performing the 
experiment at two spinning speeds and the peaks which do not shift will be the isotropic sites. In the case of a spin $3 / 2$ nucleus, the spinning sidebands make the spectrum even more difficult to interpret than in the high speed limit. The sidebands overlap and leave almost no gaps in the overall spectrum. Additionally, the total number of singularities, whose positions normally help to estimate the quadrupolar parameters, is greatly multiplied and cannot be used for this purpose as easily. Finally, in the case of multiple sites, spinning sidebands will make interpretation of quadrupolar spectra virtually impossible.

The source of the spinning sidebands lies in the assumption to drop the time dependent terms from the expressions for the spatial tensor under sample rotatioin (equation 2.82). This assumption, while simplifying the calculation, in many cases proves to be ?uite bad. There are a large number of papers in the literature which deal with spinning sidebands. Specifically the works by both Maricq and Waugh ${ }^{58}$ and Herzfeld and Berger $^{59}$ are illuminating for the case of spin $1 / 2$ nuclei. For quadrupolar problems the papers by Jakobsen et al., ${ }^{60,61}$ Samoson et al., $55,62,63$ and others ${ }^{64-76}$ provide good reference material. For the case of DAS in particular, the papers by Grandinetti et al. ${ }^{52}$ and Sun et al $^{49}$ both give a good description of the spinning sideband problem.

i'o describe spinning sidebands in spin $1 / 2$ systems, it is necessary to return to our original equation for the chemical shift anisotropy energy eigenvalues under spinning conditions.

$$
\begin{aligned}
& \Delta E^{C S A}=\hbar \omega_{l} \delta_{i s o, c s}+ \\
& \hbar \omega_{l} \delta^{C S} \sqrt{\frac{2}{3}} \sum_{m=-2}^{2} \sum_{n=-2}^{2} D_{m 0}^{(2)}\left(\omega_{r} t+\phi_{r}, \theta, 0\right) D_{n m}^{(2)}\left(\alpha^{C S}, \beta^{C S}, \gamma^{C S}\right) \rho_{2 n}^{C S}
\end{aligned}
$$

This expression may be written alternatively below 


$$
\begin{gathered}
\Delta E^{C S A}=\hbar \sum_{m=-2}^{2} W_{m}\left(\alpha^{C S}, \beta^{C S}, \theta\right) e^{-i m\left(\omega_{r} t+\phi_{r}+\gamma^{C S}\right)} \\
W_{0}\left(\alpha^{C S}, \beta^{C S}, \theta\right)=\omega_{l} \delta_{i s o, c s}+\sqrt{\frac{2}{3}} \omega_{l} \delta^{C S} d_{00}^{(2)}(\theta) \sum_{n=-2}^{2} e^{-i n \alpha^{C S}} d_{n 0}^{(2)}\left(\beta^{C S}\right) \rho_{2 n}^{C S} . \\
W_{m}\left(\alpha^{C S}, \beta^{C S}, \theta\right)=\sqrt{\frac{2}{3}} \omega_{l} \delta^{C S} d_{m 0}^{(2)}(\theta) \sum_{n=-2}^{2} e^{-i n \alpha^{C S}} d_{n m}^{(2)}\left(\beta^{C S}\right) \rho_{2 n}^{C S}
\end{gathered}
$$

This expression allows us to write the time domain free induction decay following a pulse.

$$
\begin{aligned}
\phi^{C S}\left(t_{2}\right)= & \frac{1}{\hbar} \int_{0}^{t_{2}} \Delta E^{C S A} d t \\
= & W_{0}\left(\alpha^{C S}, \beta^{C S}, \theta\right) t_{2}- \\
& \sum_{m \neq 0} \frac{W_{m}\left(\alpha^{C S}, \beta^{C S}, \theta\right)}{i m \omega_{r}}\left(e^{-i m\left(\omega_{r} t_{2}+\phi_{r}+\gamma^{C S}\right)}-e^{-i m\left(\phi_{r}+\gamma^{C S}\right)}\right) \\
S\left(t_{2}\right)= & e^{-i \phi C S}\left(t_{2}\right) \\
= & e^{-i W_{0}\left(\alpha^{C S}, \beta^{C S}, \theta\right) t_{2}} \\
& \times \exp \left(\sum_{m \neq 0} \frac{W_{m}\left(\alpha^{C S}, \beta^{C S}, \theta\right)}{m \omega_{r}}\left(e^{-i m\left(\omega_{r} t_{2}+\phi_{r}+\gamma^{C S}\right)}-e^{-i m\left(\phi_{r}+\gamma^{C S}\right)}\right)\right)
\end{aligned}
$$

Now we may use Dirac delta functions $\delta(z)$ (see chapter 2) to rewrite 3.12 below.

$$
\begin{aligned}
S\left(t_{2}\right)= & e^{-i W_{0}\left(\alpha^{C S}, \beta^{C S}, \theta\right) t_{2}} \\
& \times \frac{1}{2 \pi} \int_{0}^{2 \pi} \delta\left(\psi-\omega_{r} t_{2}-\phi_{r}-\gamma^{C S}\right) \exp \left(\sum_{m \neq 0} \frac{W_{m}\left(\alpha^{C S}, \beta^{C S}, \theta\right)}{m \omega_{r}} e^{-i m \psi}\right) d \psi \\
& \times \frac{1}{2 \pi} \int_{0}^{2 \pi} \delta\left(\psi-\phi_{r}-\gamma^{C S}\right) \exp \left(-\sum_{m \neq 0} \frac{w_{m}\left(\alpha^{C S}, \beta^{C S}, \theta\right)}{m \omega_{r}} e^{-i m \psi}\right) d \psi \\
\delta(z)= & \frac{1}{2 \pi} \sum_{m=-\infty}^{\infty} \exp (-i m z)
\end{aligned}
$$

The alternative series expansion definition of delta functions (given in equation 2.90) allows us to write $S\left(t_{2}\right)$ in a different fashion (3.14). 


$$
\begin{aligned}
S\left(t_{2}\right) & =\exp \left(-i W_{0}\left(\alpha^{C S}, \beta^{C S}, \theta\right) t_{2}\right) \\
& \times \sum_{N_{1}} \frac{1}{2 \pi} \int_{0}^{2 \pi} \exp \left(i N_{1}\left(\psi-\omega_{r} t_{2}-\phi_{r}-\gamma^{C S}\right)+\sum_{m \neq 0} \frac{w_{m}\left(\alpha^{C S}, \beta^{C S}, \theta\right)}{m \omega_{r}} e^{-i m \psi}\right) d \psi \\
& \times \sum_{N_{2}} \frac{1}{2 \pi} \int_{0}^{2 \pi} \exp \left(i N_{2}\left(\psi-\phi_{r}-\gamma^{C S}\right)-\sum_{m \neq 0} \frac{W_{m}\left(\alpha^{C S}, \beta^{C S}, \theta\right)}{m \omega_{r}} e^{-i m \psi}\right) d \psi
\end{aligned}
$$

This expression may be simplified by reversing the summation over $N_{2}$ and pulling the $\psi$ independent terms out of the integrals.

$$
\begin{aligned}
S\left(t_{2}\right) & =e^{-i W_{0}\left(\alpha^{C S}, \beta^{C S}, \theta\right) t_{2}} \sum_{N_{1}, N_{2}} A_{N_{1}} A_{N_{2}}^{*} e^{-i\left(N_{1} \omega_{r} t_{2}+\left(N_{1}-N_{2}\right)\left(\phi_{r}+\gamma^{C S}\right)\right)} \\
A_{N} & =\frac{1}{2 \pi} \int_{0}^{2 \pi} \exp \left(i N \psi+\sum_{m \neq 0} \frac{W_{m}\left(\alpha^{C S}, \beta^{C S}, \theta\right)}{m \omega_{r}} e^{-i m \psi}\right) d \psi \\
A_{N}^{*} & =\frac{1}{2 \pi} \int_{0}^{2 \pi} \exp \left(-i N \psi-\sum_{m \neq 0} \frac{W_{m}\left(\alpha^{C S}, \beta^{C S}, \theta\right)}{m \omega_{r}} e^{-i m \psi}\right) d \psi
\end{aligned}
$$

Since all possible crystaliite orientations are present in a powder sample, the signal may be simplified by averaging over the $\left(\phi_{r}+\gamma^{C S}\right)$ variables.

$$
\left\langle S\left(t_{2}\right)\right\rangle_{\left(\phi_{r}+\gamma^{C S}\right)}=e^{-i W_{0}\left(\alpha^{C S}, \beta^{C S}, \theta\right) t_{2}} \sum_{N_{1}=-\infty}^{\infty} A_{N_{1}} A_{N_{1}}^{*} e^{-i N_{1} \omega_{r} t_{2}}
$$

The final step is to do the powder average over the remaining two Euler angles, $\left(\alpha^{C S}, \beta^{C S}\right)$ (see section at the end of chapter 2 for a discussion powder averages). In the case of magic-angle spinning, the first exponential term has no orientational dependence, and the signal is given below.

$$
\begin{aligned}
\left\langle S\left(t_{2}\right)\right\rangle_{M A S} & =e^{-i \omega_{1} \delta_{\text {iso.cs }} t_{2}} \sum_{N_{1}=-\infty}^{\infty} S_{N_{1}} e^{-i N_{1} \omega_{r} t_{2}} \\
S_{N} & =\frac{1}{4 \pi} \int_{0}^{2 \pi} \int_{0}^{\pi}\left|A_{N}\right|^{2} \sin \beta^{C S} d \beta^{C S} d \alpha C S
\end{aligned}
$$

This MAS signal shows that there will exist a set of $N$ spinning sidebands a distance $N \omega_{r}$ from the isotropic peak with intensities given by $S_{N}$. In practice, the $S_{N}$ will die away 
fairly quickly with increasing $N$. In fact, once $N \omega_{r}$ is outside of the static powder pattern, $S_{N}$ will be nearly zero (but not absolutely zero). This behavior is seen in the slow speed MAS spectrum for a spin 1/2 nucleus in figure 3.6.

For the case of quadrupolar nuclei, this analysis may again be performed. For the first-order quadrupolar interaction, the math is entirely identical, except that signal must be added together for all of the possible single quantum transitions. The second order quadrupolar interaction presents a more difficult problem. Remember that the expression for the second-order quadrupolar energy splitting is given below (identical to equation 2.97)

$$
\Delta E^{(2 Q)}=\frac{\hbar \omega_{Q}^{2}}{\omega_{l}}\left(I(I+1)-\frac{3}{4}\right)\left(2 A_{21}^{Q} A_{2-1}^{Q}+A_{22}^{Q} A_{2-2}^{Q}\right)
$$

This expression may be simplified using the following tensor relationship for products of tensor elements (3.19).

$$
\begin{aligned}
A_{2 m} A_{2-m} & =\sum_{l=0,2,4}\langle l, 0 \mid 2,2, m,-m\rangle a_{l 0} \\
a_{l 0} & =\sum_{n} D_{n 0}^{(l)}\left(\omega_{r} t+\phi_{r}, \theta, 0\right) \sum_{k} D_{k n}^{(l)}(\alpha, \beta, \gamma) \sigma_{l k} \\
\sigma_{l k} & =\sum_{j}\langle l, k \mid 2,2, j, k-j\rangle \rho_{2 j} \rho_{2 k-j}
\end{aligned}
$$

Here the $a_{l 0}$ tensor has been explicitly written out for rotation from the PAS to the rotor frame followed by rotation from the rotor frame to the LAB frame. The coefficients $\left\langle L, M \mid l, l^{\prime}, m, M-m\right\rangle$ used in the expansion are the usual Clebsh-Gordon coefficients. For the quadrupolar interaction, this expansion leads to the following formula for the second-order splitting. 


$$
\begin{aligned}
\Delta E^{(2 Q)} & =\frac{\hbar \omega_{Q}^{2}}{\omega_{l}} 2\left(I(I+1)-\frac{3}{4}\right) \sum_{l=0,2,4} \sum_{m>0} \frac{\langle l, 0 \mid 2,2, m,-m\rangle}{m} a_{l 0}^{Q} \\
\sigma_{00}^{Q} & =\frac{3 e^{2} q^{2}}{2 \sqrt{5}}\left(\frac{\eta_{Q}^{2}}{3}+1\right) \\
\sigma_{20}^{Q} & =\frac{3 e^{2} q^{2}}{2} \sqrt{\frac{2}{7}}\left(\frac{\eta_{Q}^{2}}{3}-1\right), \quad \sigma_{2 \pm 2}^{Q}=\frac{3 e^{2} q^{2} \eta_{Q}}{\sqrt{21}} \\
\sigma_{40}^{Q} & =\frac{9 e^{2} q^{2}}{\sqrt{70}}\left(\frac{\eta_{Q}^{2}}{18}+1\right), \quad \sigma_{4 \pm 2}^{Q}=\frac{3 e^{2} q^{2} \eta_{Q}}{2 \sqrt{7}}, \quad \sigma_{4 \pm 4}^{Q}=\frac{e^{2} q^{2} \eta_{Q}^{2}}{4}
\end{aligned}
$$

As previously, we may rewrite the energy splitting in the following fashion (just as in equation 3.10).

$$
\begin{aligned}
\Delta E^{(2 Q)} & =\hbar \sum_{n=-4}^{4} e^{-i n\left(\omega_{r} t+\phi_{r}+\gamma^{Q}\right)} W_{n}\left(\alpha^{Q}, \beta^{Q}, \theta\right) \\
W_{n}\left(\alpha^{Q}, \beta^{Q}, \theta\right) & =\frac{\omega_{Q}^{2}}{\omega_{l}} 2\left(I(I+1)-\frac{3}{4}\right) \sum_{\substack{l=0,2,4 \\
l \leq|n|}} e^{-i n\left(\omega_{r} t+\phi_{r}+\gamma^{Q}\right)} d_{n 0}^{(l)}(\theta) \\
& \times \sum_{k} e^{-i k \alpha^{Q}} d_{k n}^{(l)}\left(\beta^{Q}\right) \sigma_{l k}^{Q} \sum_{m>0} \frac{\langle l, 0 \mid 2,2, m,-m\rangle}{m}
\end{aligned}
$$

It is important to note the similarity between this equation and equation 3.11 . In fact, the same analysis may be followed to arrive at a very similar result following the average over rotor phase.

$$
\begin{gathered}
\phi^{Q}\left(t_{2}\right)=\frac{1}{\hbar} \int_{0}^{t_{2}} \Delta E^{(2 Q)} d t \\
S\left(t_{2}\right)=e^{-i \phi^{Q}\left(t_{2}\right)} \\
\left\langle S\left(t_{2}\right)\right\rangle_{\left(\phi_{r}+\gamma^{Q}\right)}=e^{-i W_{0}\left(\alpha^{Q}, \beta^{Q}, \theta\right) t_{2}} \sum_{N_{1}=-\infty}^{\infty} A_{N_{1}} A_{N_{1}}^{*} e^{-i N_{1} \omega_{r} t_{2}} \\
A_{N}=\frac{1}{2 \pi} \int_{0}^{2 \pi} \exp \left(i N \psi+\sum_{n \neq 0} \frac{W_{n}\left(\alpha^{Q}, \beta^{Q}, \theta\right)}{n \omega_{r}} e^{-i n \psi}\right) d \psi \\
A_{N}^{*}=\frac{1}{2 \pi} \int_{0}^{2 \pi} \exp \left(-i N \psi-\sum_{n \neq 0} \frac{W_{n}\left(\alpha^{Q}, \beta^{Q}, \theta\right)}{n \omega_{r}} e^{-i n \psi}\right) d \psi
\end{gathered}
$$


This again shows that all of the sidebands will appear at a frequency $N \omega_{r}$ from the centerband with positive intensity given by $\left|A_{N}\right|^{2}$. In this case, the averaging over the final two Euler angles may not be performed analytically, since the second-order quadrupolar frequencies are anisotropic under all single axis spinning angles. The result of a complete powder average is to generate spectra much like the slow speed spin-1/2 MAS spectrum in figure 3.6 except that instead of narrow isotropic lines there will be miniature powder patterns as seen in the same figure. With the above equations, simulations of spinning sidebands may be accomplished with methods similar to those described at the end of chapter 2. There are faster methods, however, for simulating sideband intensities and I would direct the reader to various papers on this and related subjects. ${ }^{49.52,58-60,64,74,76-81}$

Finally, suppose the spinning angle $\theta$ is set to $0^{\circ}$, or parallel to the static magnetic field. In this case, all of the $W_{n}$ with $n \neq 0$ will be analytically zero for both the chemical shift and quadrupolar interactions. This means that spinning the sample parallel to the magnetic field has absolutely no effect on the spectrum (relative to a static experiment) and generates no spinning sidebands. This feature will be useful in the next section when the $k=5$ DAS experiment is described, as one of thr spinning angles is indeed $0^{\circ}$.

The dynamic angle spinning experiment may be analyzed in a very similar manner as the previous two cases. The first step is to redefine the time axes in the normal DAS experiment. In figure 3.8, the new time definitions are shown along with the original DAS sequence. Notice that the only difference is that the evolution between the first two $\pi / 2$ pulses is defined as $t_{1}$ rather than $t_{1} /(k+1)$ and the $t_{2}$ evolution begins immediately following the last $\pi / 2$ pulse. This definition of time axes differs from the original DAS experiment only in the application of a shearing transformation following the twodimensional Fourier transform. The shearing angle is related to the $k$ value by the following equation.

$$
\theta_{s}=\tan ^{-1} k
$$


Shearing transformations are well known in $\mathrm{NMR}^{82-86}$ and will not be discussed at this point. The two dimensional DAS experiment performed with $k=1\left(37.38^{\circ}, 79.19^{\circ}\right)$ on

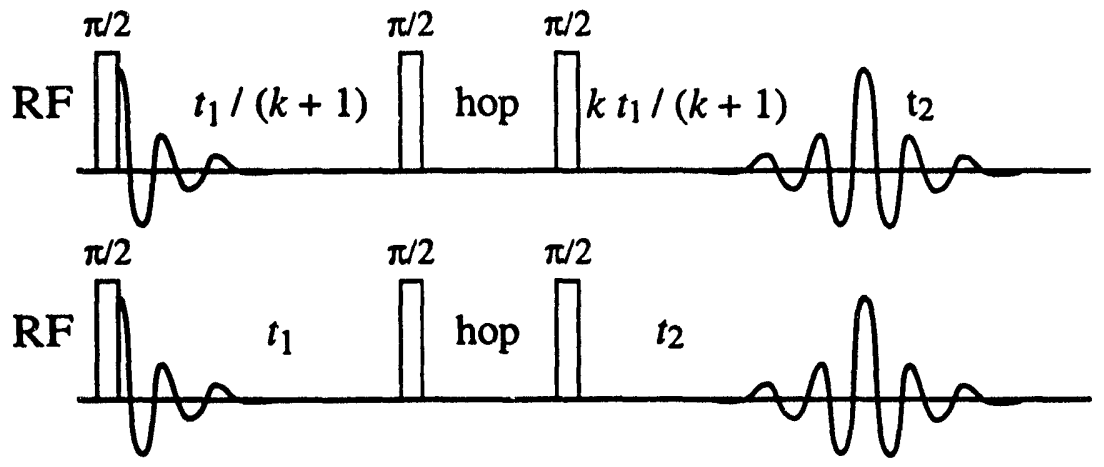

Figure 3.8 Redefined DAS Pulse Sequence for Spinning Sideband Calculation. The time definitions given above are useful for calculating sideband positions and intensities and the original style DAS spectrum may be arrived at by shearing the final two-dimensional Fourier transformed data set.

$\mathrm{RbClO}_{4}$ is shown in figure 3.9. The $\pi / 2$ selective pulse widths were $5.0 \mu \mathrm{s}$ and the spinning speed was $3.2 \mathrm{kHz}$. The data was taken at a magnetic field strength of $11.7 \mathrm{~T}$ and was sheared with a $45^{\circ}$ shearing transformation. Notice, the shearing transformation creates a spectrum with isotropic peaks and spinning sidebands in the $\omega_{1}$ (DAS) dimension and anisotropic $79.19^{\circ}$ slow spinning VAS spectra in the $\omega_{2}$ dimension. The positions of the spinning sidebands in the projection onto the $\omega_{1}^{\prime}$ DAS axis in figure 3.9 are at the isotropic frequency plus or minus one half the spinning speed. The factor of one half, while initially appearing rather unusual, may be explained by looking at the actual sideband positions with the same formalism used previously. ${ }^{52}$ As a starting point, we will assume that energy splitting will be determined by only the quadrupolar interaction (no CSA present for now, however the results may be easily generalized) given in equation 3.20. In this case the evolved phase may be written as the sum of two integrals, given in equation 3.24. 


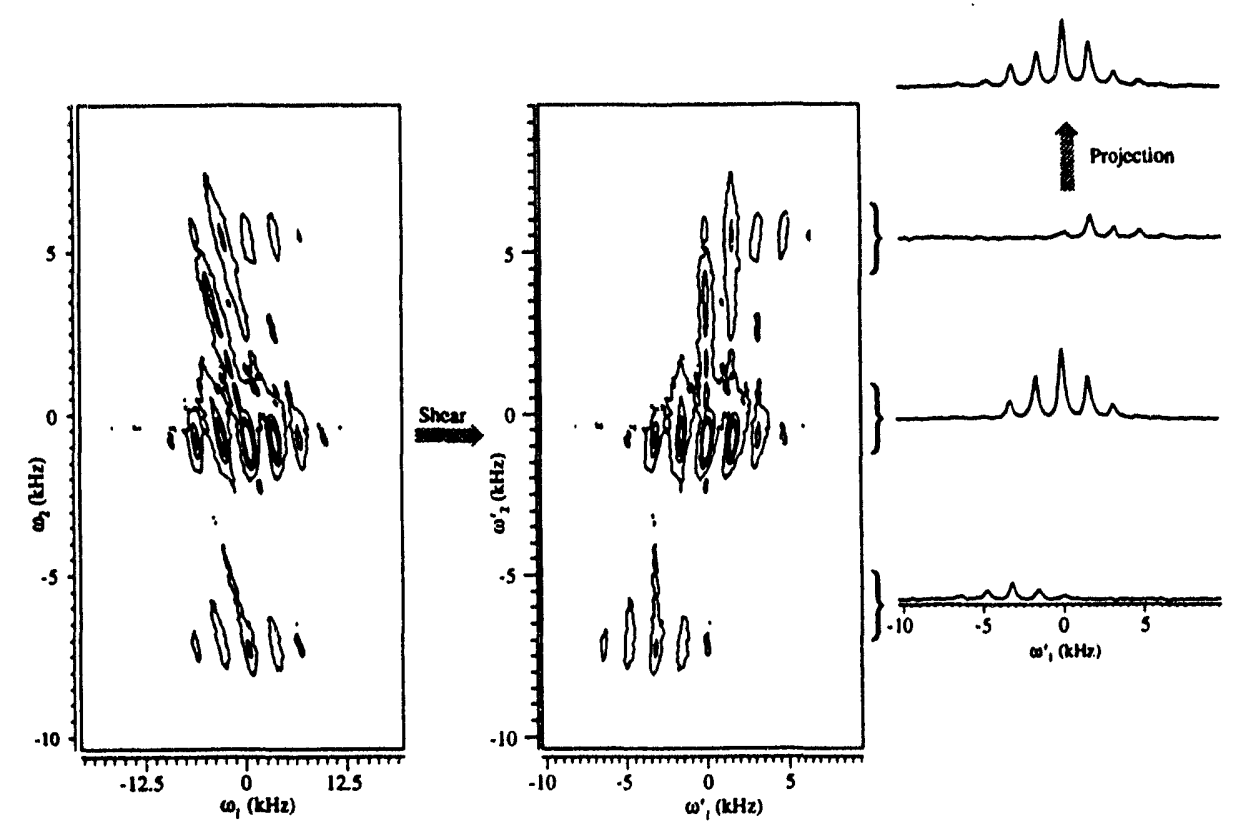

Figure 3.9 Sidebands in $k=1$ DAS 2D Spectrum of $\mathrm{RbClO}_{4}$ at $11.7 \mathrm{~T}$. The pulse widths were $5.0 \mu \mathrm{s}$ and spinning speed was $3.2 \mathrm{kHz}$. The data was sheared with a $45^{\circ}$ shearing transformation following data collection and processing with the sequence in figure 3.6.

$$
\begin{aligned}
\phi^{D A S}\left(t_{1}, t_{2}\right) & =\frac{1}{\hbar} \int_{0}^{t_{1}} \Delta E^{(2 Q)}\left(\alpha^{Q}, \beta^{Q}, \theta_{1}, t, \phi_{r 1}\right) d t \\
& +\frac{1}{\hbar} \int_{0}^{t_{2}} \Delta E^{(2 Q)}\left(\alpha^{Q}, \beta^{Q}, \theta_{2}, t, \phi_{r 2}\right) d t
\end{aligned}
$$

The variables in the expressions for the energy splitting indicate that we will consider both the absolute rotor phase and PAS orientation of the sample. Upon performing these integrals, the DAS signal may be expressed below.

$$
\begin{aligned}
\phi^{D A S}\left(t_{1}, t_{2}\right)= & W_{0}\left(\alpha^{Q}, \beta^{Q}, \theta\right) t_{1}+W_{0}\left(\alpha^{Q}, \beta^{Q}, \theta\right) t_{2} \\
& -\sum_{m \neq 0} \frac{W_{m}\left(\alpha^{Q}, \beta^{Q}, \theta_{1}\right)}{i m \omega_{r}}\left(e^{-i m\left(\omega_{r} t_{1}+\phi_{r 1}+\gamma^{Q}\right)}-e^{-i m\left(\phi_{r 1}+\gamma^{Q}\right)}\right) \\
& -\sum_{m \neq 0} \frac{W_{m}\left(\alpha^{Q}, \beta^{Q}, \theta_{2}\right)}{i m \omega_{r}}\left(e^{-i m\left(\omega_{r} t_{2}+\phi_{r 2}+\gamma^{Q}\right)}-e^{-i m\left(\phi_{r 2}+\gamma^{Q}\right)}\right)
\end{aligned}
$$




$$
\begin{aligned}
S\left(t_{1}, t_{2}\right)= & e^{-i \phi^{D A S}\left(t_{1}, t_{2}\right)} \\
& =e^{-i W_{0}\left(\alpha^{Q}, \beta^{Q}, \theta_{1}\right) t_{1}} e^{-i W_{0}\left(\alpha^{Q}, \beta^{Q}, \theta_{2}\right) t_{2}} \\
& \times \exp \left(\sum_{m \neq 0} \frac{W_{m}\left(\alpha^{Q}, \beta^{Q}, \theta_{1}\right)}{m \omega_{r}}\left(e^{-i m\left(\omega_{r} t_{1}+\phi_{r 1}+\gamma^{Q}\right)}-e^{-i m\left(\phi_{r 1}+\gamma^{Q}\right)}\right)\right) \\
& \times \exp \left(\sum_{m \neq 0} \frac{W_{m}\left(\alpha^{Q}, \beta^{Q}, \theta_{2}\right)}{m \omega_{r}}\left(e^{-i m\left(\omega_{r} t_{2}+\phi_{r 2}+\gamma^{Q}\right)}-e^{-i m\left(\phi_{r 2}+\gamma^{Q}\right)}\right)\right)
\end{aligned}
$$

This can again be simplified with the use of delta functions as before to give the following equation for the DAS NMR signal.

$$
\begin{aligned}
S\left(t_{1}, t_{2}\right)= & e^{-i W_{0}\left(\alpha^{Q}, \beta^{Q}, \theta_{1}\right) t_{1}-i W_{0}\left(\alpha^{Q}, \beta^{Q}, \theta_{2}\right) t_{2}} \\
& \times \sum_{N_{1}, N_{2}, N_{3}, N_{4}} A_{N_{1}}\left(\theta_{1}\right) A_{N_{2}}^{*}\left(\theta_{1}\right) A_{N_{3}}\left(\theta_{2}\right) A_{N_{4}}^{*}\left(\theta_{2}\right) \\
& \times e^{-i\left[\begin{array}{l}
\left.\left(N_{1}-N_{2}+N_{3}-N_{4}\right)\left(\phi_{r 1}+\gamma^{Q}\right)+\left(N_{3}-N_{4}\right)\left(\phi_{r 2}-\phi_{r 1}\right)\right] \\
+\left(N_{1}+N_{3}-N_{4}\right) \omega_{r} t_{1}+N_{3} \omega_{r} t_{2}
\end{array}\right]} \\
A_{N}(\theta) & =\frac{1}{2 \pi} \int_{0}^{2 \pi} \exp \left(i N \psi+\sum_{m \neq 0} \frac{W_{m}\left(\alpha^{Q}, \beta^{Q}, \theta\right)}{m \omega_{r}} e^{-i m \psi}\right) d \psi
\end{aligned}
$$

This may be averaged over the initial rotor phase, $\left(\phi_{r 1}+\gamma^{Q}\right)$, as before.

$$
\begin{aligned}
\left\langle S\left(t_{1}, t_{2}\right)\right\rangle_{\phi_{r 1}, \gamma^{Q}}= & e^{-i W_{0}\left(\alpha^{Q}, \beta^{Q}, \theta_{1}\right) t_{1}-i W_{0}\left(\alpha^{Q}, \beta^{Q}, \theta_{2}\right) t_{2}} \\
& \times \sum_{N_{1}, N_{2}, N_{3}} A_{N_{1}}\left(\theta_{1}\right) A_{N_{2}}^{*}\left(\theta_{1}\right) A_{N_{3}}\left(\theta_{2}\right) A_{N_{1}-N_{2}+N_{3}}^{*}\left(\theta_{2}\right) \\
& \times e^{-i\left[\left(N_{2}-N_{1}\right)\left(\phi_{r 2}-\phi_{r 1}\right)+N_{2} \omega_{r} t_{1}+N_{3} \omega_{r} t_{2}\right]}
\end{aligned}
$$

In most cases, the relative phase of the rotor $\left(\phi_{r 2}-\phi_{r 1}\right)$ between the first and second evolution periods will be relatively random. In the case of large numbers of scans, these variables $\left(\phi_{r 2}-\phi_{r 1}\right)$ may be averaged over as well.

$$
\begin{aligned}
\left\langle S\left(t_{1}, t_{2}\right)\right\rangle_{\phi_{r 1}, \phi_{r 2}, \gamma^{Q}} & =e^{-i W_{0}\left(\alpha^{Q}, \beta^{Q}, \theta_{1}\right) t_{1}-i W_{0}\left(\alpha^{Q}, \beta^{Q}, \theta_{2}\right) t_{2}} \\
& \times \sum_{N_{1}, N_{2}}\left|A_{N_{1}}\left(\theta_{1}\right)\right|^{2}\left|A_{N_{2}}\left(\theta_{2}\right)\right|^{2} e^{-i\left[N_{1} \omega_{r} t_{1}+N_{2} \omega_{r} t_{2}\right]}
\end{aligned}
$$


This indicates that the intensity of all of the sidebands in the two dimensional spectrum will be positive. The peaks will occur at frequencies $N_{1} \omega_{r}$ from $W_{0}\left(\theta_{1}\right)$ in the first dimension correlated with frequencies at $N_{2} \omega_{r}$ from $W_{0}\left(\theta_{2}\right)$ in the second. When the spectrum is sheared, the peaks will all remain positive, however their positions will shift. Transforming the time variables into the sheared time definitions, we will arrive at the following expression for the DAS signal.

$$
\begin{aligned}
t_{1} & =\frac{t_{1}^{\prime}}{k+1} \\
t_{2} & =\frac{k t_{1}^{\prime}}{k+1}+t_{2}^{\prime} \\
\left\langle S\left(t_{1}^{\prime}, t_{2}^{\prime}\right)\right\rangle= & e^{-i \frac{\left(W_{0}\left(\alpha^{Q}, \beta^{Q}, \theta_{1}\right)+k W_{0}\left(\alpha^{Q}, \beta^{Q}, \theta_{2}\right)\right) t_{1}^{\prime}}{k+1}} e^{-i W_{0}\left(\alpha^{Q}, \beta^{Q}, \theta_{2}\right) t_{2}^{\prime}} \\
& \times \sum_{N_{1}, N_{2}}\left|A_{N_{1}}\left(\theta_{1}\right)\right|^{2}\left|A_{N_{2}}\left(\theta_{2}\right)\right|^{2} e^{-i\left[\frac{N_{1} \omega_{r} r_{1}^{\prime}}{k+1}+\frac{k N_{2} \omega_{r} r_{1}^{\prime}}{k+1}+N_{2} \omega_{r} r_{2}^{\prime}\right]}
\end{aligned}
$$

The definition of the DAS angle pairs is equivalent to the following equation.

$$
W_{0}\left(\alpha^{Q}, \beta^{Q}, \theta_{1}\right)+k W_{0}\left(\alpha^{Q}, \beta^{Q}, \theta_{2}\right)=(k+1) \omega_{i s o}^{(2 Q)}
$$

Which reduces equation 3.29 to the form in equation 3.31 .

$$
\begin{aligned}
\left\langle S\left(t_{1}^{\prime}, t_{2}^{\prime}\right)\right\rangle= & e^{-i \omega_{i s o}^{(2 Q)} t_{1}^{\prime}} e^{-i W_{0}\left(\alpha^{Q}, \beta^{Q}, \theta_{2}\right) t_{2}^{\prime}} \\
& \times \sum_{N_{1}, N_{2}}\left|A_{N_{1}}\left(\theta_{1}\right)\right|^{2}\left|A_{N_{2}}\left(\theta_{2}\right)\right|^{2} e^{-i\left[\frac{N_{1} \omega_{r} r_{1}^{\prime}}{k+1}+\frac{k N_{2} \omega_{r} r_{1}^{\prime}}{k+1}+N_{2} \omega_{r} r_{2}^{\prime}\right]} \\
\left\langle S\left(t_{1}^{\prime}, 0\right)\right\rangle= & e^{-i \omega_{i s o}^{(2 Q)} t_{1}^{\prime}} \sum_{N_{1}, N_{2}}\left|A_{N_{1}}\left(\theta_{1}\right)\right|^{2}\left|A_{N_{2}}\left(\theta_{2}\right)\right|^{2} e^{-i\left[\frac{N_{1}}{k+1}+\frac{k N_{2}}{k+1}\right] \omega_{r} r_{1}^{\prime}}
\end{aligned}
$$

This equation shows that the isotropic spectrum arrived at by Fourier transforming the DAS echo tops at $t_{2}=0$ will have sidebands at multiples of two frequencies, $k \omega_{r} /(k+1)$ and $\omega_{r} /(k+1)$. The two dimensional spectrum will have sidebands at multiples of the same two frequencies in $\omega_{1}$ and at $\omega_{r}$ in $\omega_{2}$. Looking again at the two dimensional DAS spectrum in figure 3.9 we observe exactly these sideband positions. Each of the slices ex- 
tracted on the right corresponds to the isotropic peak and sidebands where $N_{1}=0, \pm 1, \pm 2$, \pm 3 , etc for $N_{2}=-2,0$ and +2 respectively. It is interesting also that the $N_{2}=-1$ and +1

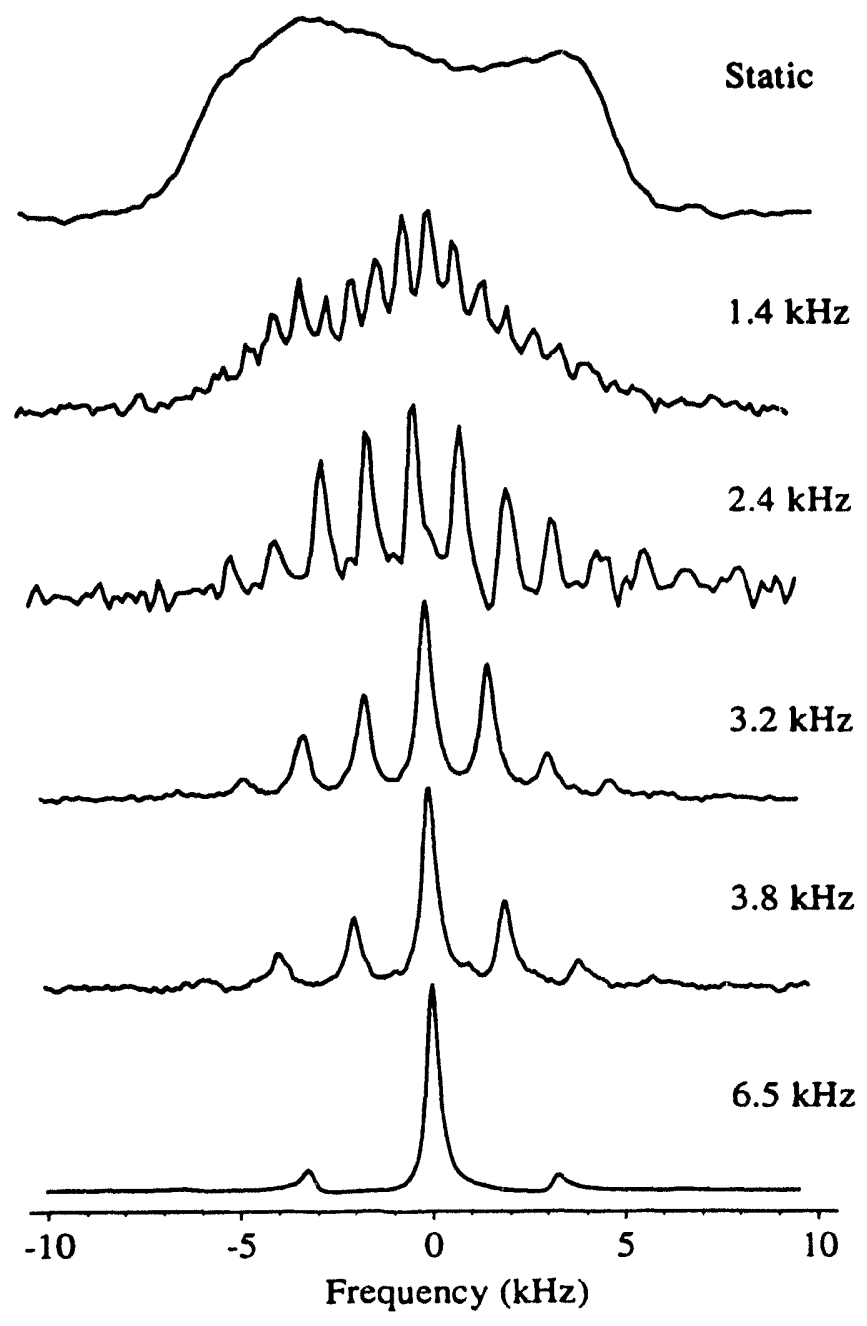

Figure $3.101 \mathrm{D}^{87} \mathrm{RbClO}_{4}$ DAS Spectra at a Variety of Spinning Speeds;. The $k=1$ DAS spectra are shown over a range of spinning speeds. The intensity of the sidebands may be described by equation 3.30 .

slices have very low intensity as the detection angle $\left(79.19^{\circ}\right)$ is nearly $90^{\circ}$ where odd order sidebands may be shown to have no intensity. Also, it may be seen that the most intense peak in each of these slices corresponds to the $N_{1}=0$ peak. The sidebands in each of the slices are separated by $\omega_{r} /(k+1)$ which in this case is $\omega_{r} / 2$. 
Figure 3.10 shows the one-dimensional DAS spectrum of ${ }^{87} \mathrm{RbClO}_{4}$ taken at 11.7T at a variety of spinning rates for the usual $k=1$ case just as in figure 3.9. The sideband intensities are seen to grow more numerous and intense as the spinning speed is reduced. The intensity of each sideband is derived from equation 3.31 by adding together the intensity (see equation 3.33) from each $N_{1}, N_{2}$ pair which contributes an integrated intensity of $\frac{1}{4 \pi} \int_{0}^{2 \pi} \int_{0}^{\pi}\left|A_{N_{1}}\left(\theta_{1}\right)\right|^{2}\left|A_{N_{2}}\left(\theta_{2}\right)\right|^{2} \sin \beta^{Q} d \beta^{Q} d \alpha^{Q}$ at a given sideband position $\left(\frac{N_{1}}{k+1}+\frac{k N_{2}}{k+1}\right) \omega_{r}$ from the centerband (keeping in mind that there may be degeneracies when $k$ is an integer). As is the case with double rotation (DOR, see chapter 6), the spinning sideband intensities in DAS do not necessarily approximate the static powder pattern in the limit of very slow spinning as is the case in slow spinning MAS.

When one of the spinning angles is $0^{\circ}$, as in the case of $k=5$ DAS, the formula for the DAS signal is simplified further. Since all $W_{n}\left(0^{\circ}\right)$ with $n \neq 0$ are zero, the value of the intensity integrals will be simplified. In the case where $\theta_{1}$ is $0^{\circ}$, there will be sidebands in the $\omega_{1}$ dimension of the unsheared spectrum and all sideband intensities with $N_{1}$ $\neq 0$ will be zero.

$$
\begin{aligned}
\left\langle S\left(t_{1}^{\prime}, t_{2}^{\prime}\right)\right\rangle_{\phi_{r 1}, \phi_{r 2}, \gamma^{Q}} & =e^{-i \omega_{i s o}^{(2 Q)} t_{1}^{\prime}} e^{-i W_{0}\left(\alpha^{Q}, \beta^{Q}, \theta_{2}\right) t_{2}^{\prime}} \\
& \times \sum_{N_{2}}\left|A_{N_{2}}\left(\theta_{2}\right)\right|^{2} e^{-i\left[\frac{k N_{2} \omega_{r} t_{1}^{\prime}}{k+1}+N_{2} \omega_{r} t_{2}^{\prime}\right]} \\
\left\langle S\left(t_{1}^{\prime}, 0\right)\right\rangle_{\phi_{r 1}, \phi_{r 2}, \gamma^{Q}} & =e^{-i \omega_{i s o}^{(2 Q)} t_{1}^{\prime}} \sum_{N_{2}}\left|A_{N_{2}}\left(\theta_{2}\right)\right|^{2} e^{-\frac{i k N_{2} \omega_{r} t_{1}^{\prime}}{k+1}}
\end{aligned}
$$

$\mathrm{A} k=5 \mathrm{DAS}$ spectrum is shown below in figure 3.11. The unsheared spectrum correlating the static $0^{\circ}$ spectrum with the $63.43^{\circ}$ VAS spectrum shows that there are no sidebands in the $\omega_{1}$ dimension and the sidebands are spaced by $\omega_{\mathrm{r}}$ in the $\omega_{2}$ dimension. In the sheared spectrum, the sidebands in the DAS dimension are spaced by $5 \omega_{r} / 6$ and by $\omega_{r}$ in the anisotropic spectrum. This represents the highest possible effective spinning speed in the isotropic dimension in a DAS experiment. 
In a case where the time ratio $k$ (or alternatively $1 / k$ ) is not an integer then the one dimensional isotropic projection becomes more complicated. In the case of a non-integer $k$, the sidebands at multiples of the two frequencies $k \omega_{r} /(k+1)$ and $\omega_{r} /(k+1)$ will not overlap for small integer values of $N_{1}$ and $N_{2}$. In the full two dimensional spectrum, the sidebands will appear separated, but will not overlap when projected. This sideband behavior may be seen in the $k=0.82 \mathrm{D} \mathrm{DAS}$ spectrum of $\mathrm{RbClO}_{4}$ in figure 3.12. Notice also that there are analytically no odd sidebands in the second dimension corresponding
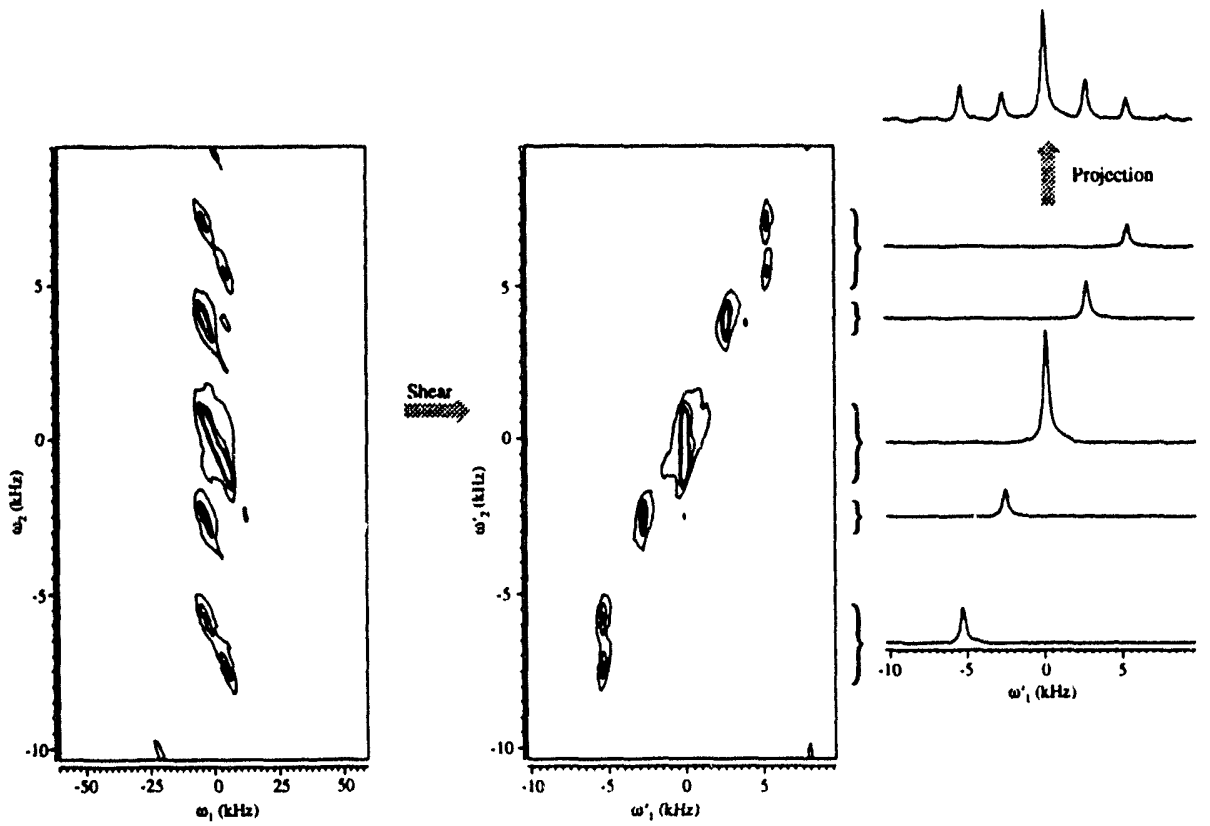

Figure $3.1{ }^{87} \mathrm{RbClO}_{4}$ Sidebands in $k=5$ DAS 2D Spectrum. The acquisition parameters are identical to those used in figure 3.7 , with the exception of the angle pair $\left(0^{\circ}\right.$ and $63.43^{\circ}$ ) used.

to odd $\mathrm{N}_{2}$. This is a direct result of spinning at $90^{\circ}$ since all odd sidebands disappear in a 1D $90^{\circ}$ VAS spectrum. 


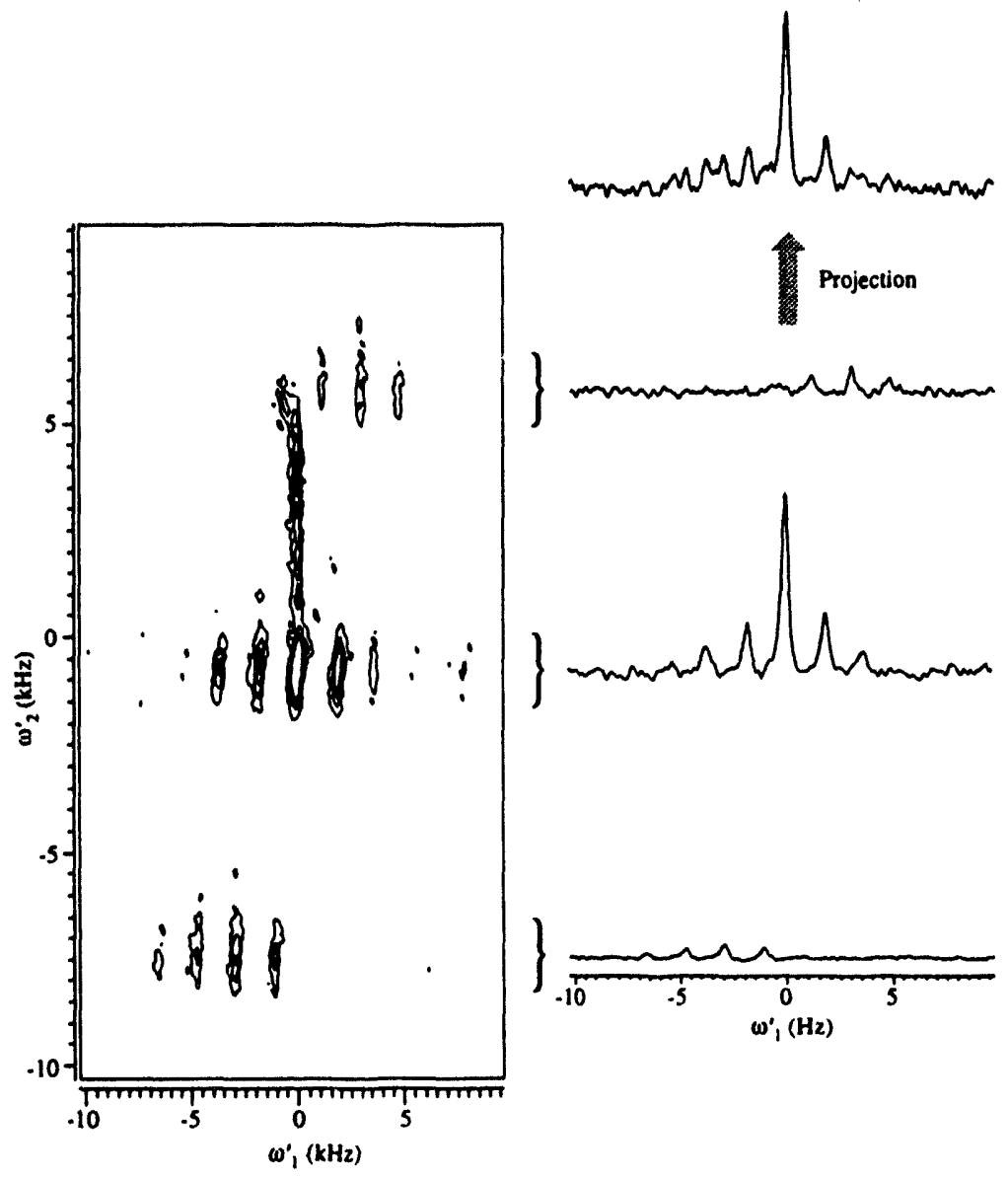

Figure $3.12{ }^{87} \mathrm{RbClO}_{4}$ Sidebands in $\mathrm{k}=0.8 \mathrm{DAS} 2 \mathrm{D}$ Spectrum. The acquisition parameters again are identical to those used previously, with the exception of the angle pair $\left(39.23^{\circ}\right.$ and $\left.90.00^{\circ}\right)$ used.

Returning to the case of one-dimensional DAS projections, the positions of sidebands are given by equation 3.32. This equation may be integrated over the final two powder angles, to yield an expression which may be calculated to generate sideband intensities in a relatively simple manner.

$$
\begin{gathered}
\left\langle S\left(t_{1}^{\prime}, 0\right)\right\rangle_{\text {powder }}=e^{-i \omega_{i s o}^{(2 Q)_{t_{1}^{\prime}}}} \sum_{N_{1}, N_{2}} S_{N_{1}, N_{2}}\left(\theta_{1}, \theta_{2}\right) e^{-i\left[\frac{N_{1}}{k+1}+\frac{k N_{2}}{k+1}\right] \omega_{r} t_{1}^{\prime}} \\
S_{N_{1}, N_{2}}\left(\theta_{1}, \theta_{2}\right)=\frac{1}{4 \pi} \int_{0}^{2 \pi} \int_{0}^{\pi}\left|A_{N_{1}}\left(\theta_{1}\right)\right|^{2}\left|A_{N_{2}}\left(\theta_{2}\right)\right|^{2} \sin \beta^{Q} d \beta^{Q} d \alpha^{Q}
\end{gathered}
$$


This expression was used to calculate spinning sideband intensities for $\mathrm{RbClO}_{4} \mathrm{DAS}$ spectra with $k$ values between 1.0 and 5.0. These simulations are shown next to the experimental spectra in figure 3.13 .

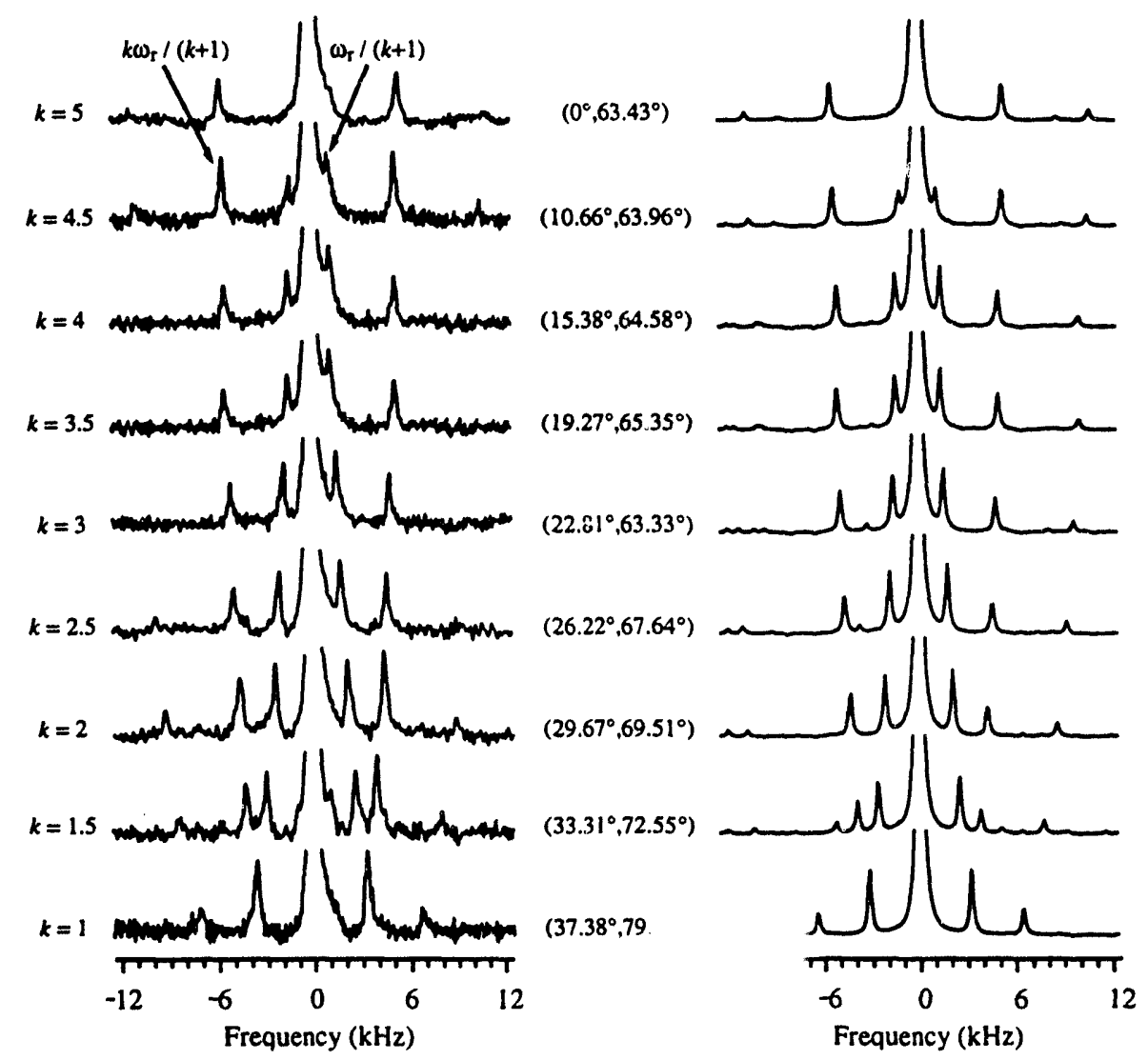

Figure 3.13 All $k$ values for fast spinning ${ }^{87} \mathrm{RbClO}_{4}$ DAS at 11.7T. These spectra were collected with experimental parameters identical to those used in the previous spectra. The simulated spectra assumed only a quadrinoiar coupling $C_{Q}$ of $3.2 \mathrm{MHz}$, an asymmetry parameter $\eta_{Q}$ of $0.10, \omega_{r}$ of $6.4 \mathrm{kHz}$ and approximately $300 \mathrm{~Hz}$ of Lorentzian broadening.

The quadrupolar parameters used to simulate the spectra were a $C_{Q}$ of $3.2 \mathrm{MHz}$, an asymmetry parameter $\eta_{Q}$ of 0.10 , and a spinning speed $\omega_{r}$ of $6.4 \mathrm{kHz}$. Lorentzian broadening was added so that the linewidths of simulated spectra were the same as the experimental spectra. It is important to note that there are basically two frequencies of spinning siciebands in these spectra, $\frac{1}{k+1} \omega_{r}$ and $\frac{k}{k+1} \omega_{r}$. In the case of $k=1$, these two frequencies are the same (just as was seen before in figure 3.9 ) and sidebands appear only at $3.2 \mathrm{kHz}$. In the case of $k=5$, the former low frequency sidebands are absent, as predicted by the- 
ory and shown earlier in figure 3.11 , and only the high frequency $5.3 \mathrm{kHz}$ sidebands appear. Because the spinning speed is quite fast compared to the second-order interaction, only the $N_{1}= \pm 1$ or $N_{2}= \pm 1$ sidebands appear in these spectra; none of the sum and difference frequencies show up.

In conclusion, the presence of spinning sidebands in DAS spectra can lead to greatly complicated spectra, with multiple spinning frequencies present. By choosing the proper value for the time ratio, $k=1$ or $k=5$, the sideband behavior is greatly simplified and the effective spinning speed is maximized. Additionally, the sideband intensities contain information which may be used to extract the quadrupolar coupling parameters. This has not been discussed here and the reader is directed to the thesis of Sun ${ }^{16}$ and related papers ${ }^{49,52}$ for additional information on simulating sideband intensities. 


\section{Linewidths in DAS (Homonuclear Dipole-Dipole Interaction)}

In this section, the contribution of the homonuclear dipole-dipole interaction to dynamic-angle spinning spectra will be discussed. Remember, the form of the static homonuclear dipolar Hamiltonian from equation 2.48 .

$$
H_{D}=-\sum_{j \neq i} \hbar \omega_{D_{i j}} \frac{1}{2}\left(3 I_{i, 0} I_{j, 0}-I_{i} \cdot I_{j}\right) d_{0,0}^{(2)}\left(\beta_{i j}^{D}\right)
$$

Under rapid spinning conditions, this may be expressed below.

$$
\begin{aligned}
H_{D} & =-\hbar \sum_{j \neq i} \omega_{D_{i j}} \frac{1}{2}\left(3 I_{i, 0} I_{j, 0}-I_{i} \cdot I_{j}\right) \sum_{m=-2}^{2} D_{m 0}^{(2)}\left(\omega_{r} t+\phi_{r}, \theta, 0\right) D_{0, m}^{(2)}\left(\alpha_{i j}^{D}, \beta_{i j}^{D}, \gamma_{i j}^{D}\right) \\
& =-\hbar d_{00}^{(2)}(\theta) \sum_{j \neq i} \omega_{D_{i j}} \frac{1}{2}\left(3 I_{i, 0} I_{j, 0}-I_{i} \cdot I_{j}\right) d_{0,0}^{(2)}\left(\beta_{i j}^{D}\right)
\end{aligned}
$$

This Hamiltonian will allow the coherence, which until this point has been assumed to be between -1 and +1 , to evolve into higher order bilinear coherences. The homonuclear dipolar contribution to the isotropic linewidth in a DAS spectrum arises since the storage pulses used during a hop cannot store bilinear terms. Also, the reduced Wigner matrix element $d_{00}^{(2)}(\theta)$ indicates that the spinning merely scales the entire interaction, under the time independent approximation (and under high speed magic-angle spinning, all dipolar couplings are scaled to zero). Since the sign of $P_{2}\left[\cos \left(\theta_{i}\right)\right]$ is reversed (see equation 3.7) following a hop from the DAS angle $\theta_{1}$ to $\theta_{2}$, and if the density matrix describing the system was the same before and after the hop, all dipolar contributions to the isotropic spectrum would be refocused at the DAS echo top. Unfortunately, the density matrix is not the same before and after the hop and the homonuclear dipolar interaction continues to dephase in the isotropic $t_{1}$ time domain, rather than refocus. An approximation which describes the dipolar dephasing of a static on-resonance homonuclear bath of spins is a Gaussian decay

$$
S(t)=\exp \left(-M_{2} t^{2} / 2\right)
$$


where $M_{2}$ is the second moment as defined by Van Vleck ${ }^{87}$. Under fast spinning VAS conditions, the effective dipolar coupling is scaled by $P_{2}\left[\cos \left(\theta_{i}\right)\right]$ and therefore the effective second moment is $M_{2} P_{2}^{2}[\cos (\theta)]$. The signal function for an on-resonance spin would then be

$$
S(t)=\exp \left(-M_{2} P_{2}^{2}(\cos \theta) t^{2} / 2\right) .
$$

Figure 3.14 shows the dipolar linewidth of both ${ }^{23} \mathrm{Na}$ and ${ }^{87} \mathrm{Rb}$ nuclei in sodium oxalate and rubidium perchlorate respectively under rapid VAS conditions. The linewidth was measured from the homogeneously broadened isotropic spectrum collected by Fourier transforming the echo tops at $t_{2}=t_{1}$ of a $90^{\circ}-t_{1}-180^{\circ}-t_{2}$ experiment (where the dwell time in $t_{1}$ was equal to the rotor period). The curves in both cases correspond to the function $\left|C_{D} P_{2}[\cos (\theta)]\right|$. where $C_{D}$ is the static homogeneous linewidth. Notice that the
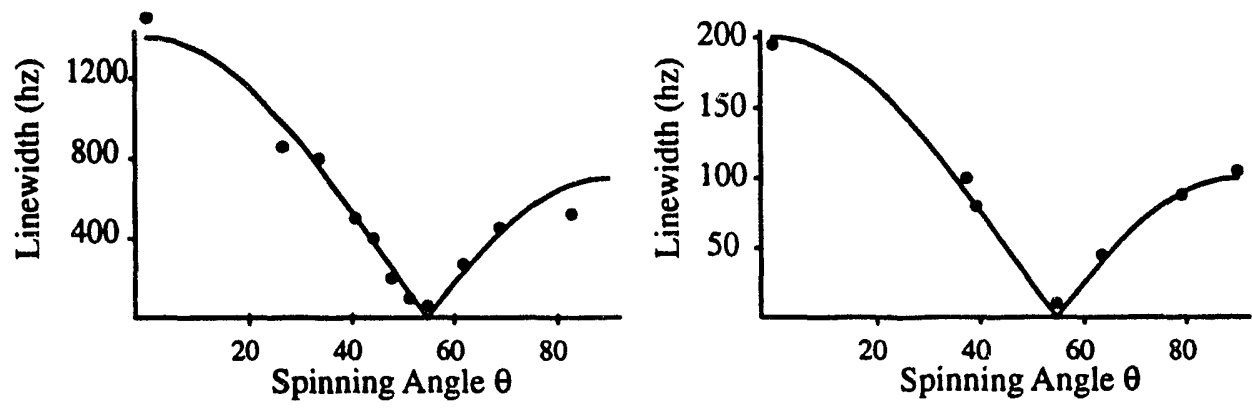

Figure 3.14 Homonuclear Dipolar Linewidth versus Spinning Angle. The left set corresponds to $\mathrm{Na}_{2} \mathrm{C}_{2} \mathrm{O}_{4}{ }^{23} \mathrm{Na}$ linewidth as a function of angle where the static linewidth is $1400 \mathrm{~Hz}$. The right set corresponds to $\mathrm{RbClO}_{4}{ }^{87} \mathrm{Rb}$ linewidth as a function of angle where the static linewidth is $195 \mathrm{~Hz}$.

linewidth goes nearly to zero at the magic angle $\left(54.74^{\circ}\right)$ in both cases. This indicates that, at a spinning speed of approximately $6 \mathrm{kHz}$, the homonuclear dipolar coupling is well described by equation 3.35 and 3.37 .

In a DAS experiment, the signal of an on-resonance spin can be expressed as the product of two Gaussian decays at two different angles. 


$$
\begin{aligned}
S\left(t_{1}\right) & =\exp \left(-M_{2} P_{2}^{2}\left(\cos \theta_{1}\right)\left(\frac{t_{1}}{k+1}\right)^{2} / 2\right) \exp \left(-M_{2} P_{2}^{2}\left(\cos \theta_{1}\right)\left(\frac{k t_{1}}{k+1}\right)^{2} / 2\right) \\
& =\exp \left(-M_{2}^{e f f} t_{1}^{2} / 2\right)
\end{aligned}
$$

For the DAS angle pairs, $\theta_{1}$ and $\theta_{2}$, the value of $P_{2}[\cos (\theta)]$ can be expressed in terms of $k$ where $P_{2}\left[\cos \left(\theta_{1}\right)\right]=\sqrt{k / 5}$ and $P_{2}\left[\cos \left(\theta_{2}\right)\right]=-\sqrt{1 / 5 k}$. This yields an effective second moment for the isotropic line in the DAS experiment of $M_{2}^{e f f}=2 k M_{2} / 5(k+1)^{2}$ giving a linewidth of approximately $\sqrt{2 k M_{2}} / \sqrt{5}(k+1)$. The narrowest line in a conventional DAS experiment should therefore arise when the $k=5$ angle pair, $0^{\circ}-63.43^{\circ}$, is used and should be about $75 \%$ of the linewidth for a $k=1$ experiment.

For the isotropic linewidth measurements, samples of sodium oxalate, $\mathrm{Na}_{2} \mathrm{C}_{2} \mathrm{O}_{4}$ and rubidium perchlorate, $\mathrm{RbClO}_{4}$, were obtained from commercial sources while the deuterated boric acid, $\mathrm{D}_{3} \mathrm{BO}_{3}$, was made by exchanging the protons in $\mathrm{H}_{3} \mathrm{BO}_{3}$ in $\mathrm{D}_{2} \mathrm{O}$, both commercially obtained. The experiments were performed at $11.7 \mathrm{~T}\left({ }^{87} \mathrm{Rb}\right.$ frequency 163.623 MHz, ${ }^{23} \mathrm{Na}$ frequency $132.201 \mathrm{MHz},{ }^{11} \mathrm{~B}$ frequency $160.446 \mathrm{MHz}$ ) with the same probe as before. The pulse sequence used for DAS was the original sequence. The selective $90^{\circ}$ times were between 4 and $8 \mu$ s and the recycle delays were between 1 and $4 \mathrm{~s}$. The spinning speeds were between 5.0 and $7.0 \mathrm{kHz}$ which effectively removed all spinning sidebands from these spectra. The spectral widths were set to $10 \mathrm{kHz}$ and between 256 and 1024 scans were acquired for each of $60 t_{1}$ points at each $k$ value.

The dipolar linewidths for $\mathrm{Na}_{2} \mathrm{C}_{2} \mathrm{O}_{4}, \mathrm{RbClO}_{4}$ and $\mathrm{D}_{3} \mathrm{BO}_{3}$ are shown in figure 3.15 for a range of $k$ values from 0.8 to 5 . It is always true that the linewidth at $k=5$ is about $20 \%$ less than at $k=1$, in agreement with the theory presented earlier. The solid curves through these data points are the best fit using the function

$$
\Delta \omega_{\text {isotropic }}=\Delta \omega_{T_{2}}+\frac{\sqrt{2 k M_{2}}}{\sqrt{5}(k+1)}
$$

where $M_{2}$ is the second moment due to homonuclear dipolar interactions in a static sample and $\Delta \omega_{T_{2}}$ is the intrinsic linewidth due to field inhomogeneity and $T_{2}$ relaxation. 
The values for $M_{2}$ extracted in this manner are very similar the those extracted from static CPMG experiments. This further confirms that the $k=5$ angle pair is the best angle pair to perform the DAS experiment.

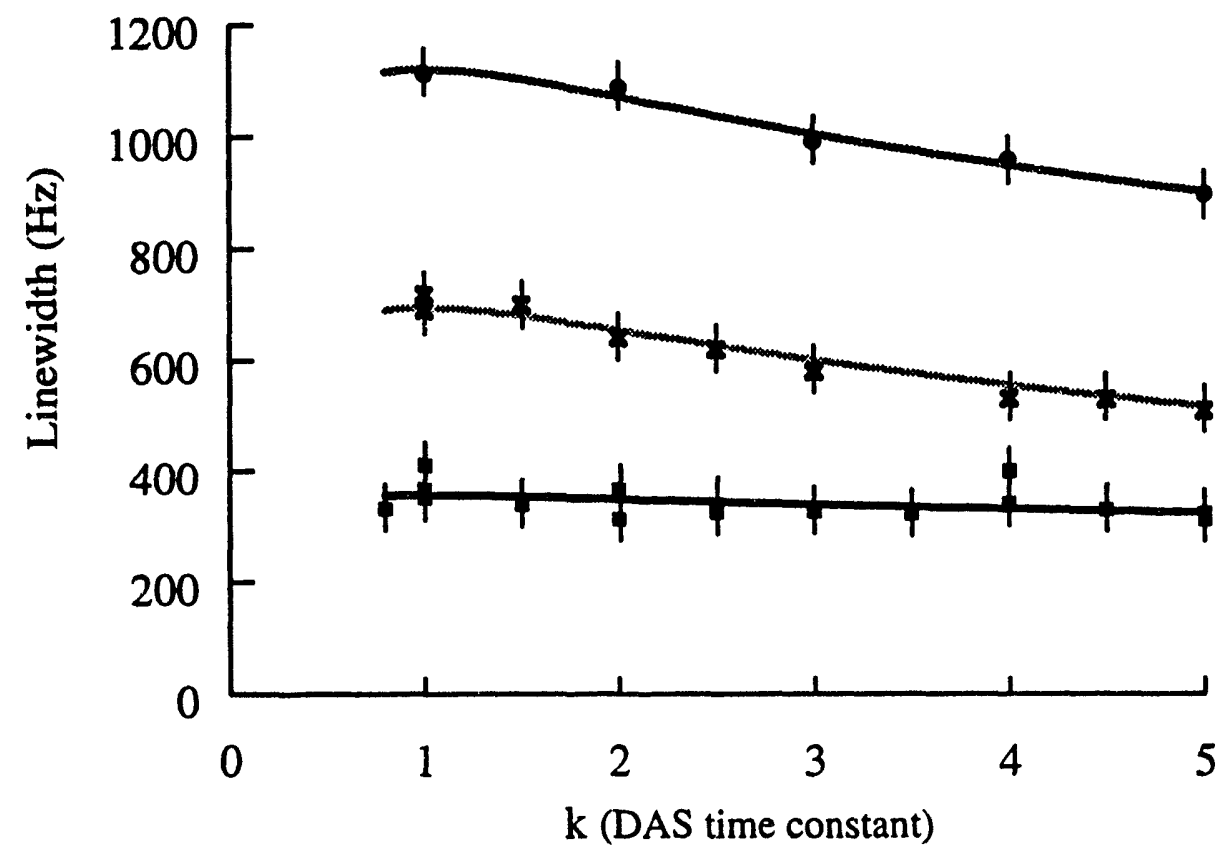

Figure 3.15 Dynamic-Angle Spinning Linewidths as a function of $k$. The solid circles are for $\mathrm{D}_{3} \mathrm{BO}_{3}$, the crosses are for $\mathrm{Na}_{2} \mathrm{C}_{2} \mathrm{O}_{4}$ and the solid boxes are for $\mathrm{RbClO}_{4}$. The lines through each set of points are the best fit with the linewidth function given by equation 3.39. 


\section{Chapter 4}

\section{Pure Phase NMR}

In traditional liquid state NMR pure-phase spectra have always been important in giving the highest signal-to-noise ratios and signal resolution. The basic problem is most easily illustrated with a simple two-dimensional data set of the form $S\left(t_{1}, t_{2}\right)$ (notice the similarity between this equation and equation 3.7 ).

$$
S\left(t_{1}, t_{2}\right)=e^{-T_{2}^{-1}\left(t_{1}+t_{2}\right)} e^{-i \Omega_{1} t_{1}} e^{-i \Omega_{2} t_{2}}
$$

Where $\Omega_{1}$ and $\Omega_{2}$ are the frequencies in each dimension for a single peak in the 2D Fourier transformed spectrum with linewidth $1 / 2 T_{2}$. When a 2D data set of this form is Fourier transformed in the $t_{2}$ dimension, the result is a data set of the form $S\left(t_{1}, \omega_{2}\right)$.

$$
S\left(t_{1}, \omega_{2}\right)=e^{-T_{2}^{-1} t_{1}} e^{-i \Omega_{1} t_{1}}\left(A\left(\omega_{2}, \Omega_{2}\right)+i D\left(\omega_{2}, \Omega_{2}\right)\right)
$$

Where $A(\omega, \Omega)$ and $D(\omega, \Omega)$ in this case are the absorptive and dispersive Lorentzian lineshape functions respectively with a peak in the $\omega$ dimension at frequency $\Omega$.

$$
\begin{aligned}
& A(\omega, \Omega)=\frac{T_{2}}{1+(\omega-\Omega)^{2} T_{2}^{2}} \\
& D(\omega, \Omega)=\frac{(\omega-\Omega) T_{2}^{2}}{1+(\omega-\Omega)^{2} T_{2}^{2}}
\end{aligned}
$$

It is immediately apparent, that the second Fourier transform will produce a very complex result, $S\left(\omega_{1}, \omega_{2}\right)$.

$$
\begin{aligned}
S\left(\omega_{1}, \omega_{2}\right)= & \left(A\left(\omega_{1}, \Omega_{1}\right)+i D\left(\omega_{1}, \Omega_{1}\right)\right)\left(A\left(\omega_{2}, \Omega_{2}\right)+i D\left(\omega_{2}, \Omega_{2}\right)\right) \\
= & \left(A\left(\omega_{1}, \Omega_{1}\right) A\left(\omega_{2}, \Omega_{2}\right)-D\left(\omega_{1}, \Omega_{1}\right) D\left(\omega_{2}, \Omega_{2}\right)\right) \\
& +i\left(A\left(\omega_{1}, \Omega_{1}\right) D\left(\omega_{2}, \Omega_{2}\right)+D\left(\omega_{1}, \Omega_{1}\right) A\left(\omega_{2}, \Omega_{2}\right)\right)
\end{aligned}
$$

This has two terms, one real and one imaginary. If this were completely pure-absorptive mode, the real term would contain $A\left(\omega_{1}, \Omega_{1}\right) A\left(\omega_{2}, \Omega_{2}\right)$ only. The term which leads to the phase-twist lineshape is the $A\left(\omega_{1}, \Omega_{1}\right) A\left(\omega_{2}, \Omega_{2}\right)-D\left(\omega_{1}, \Omega_{1}\right) D\left(\omega_{2}, \Omega_{2}\right)$ one. Figure 4.1 shows both the pure-absorption mode and phase-twist $2 \mathrm{D}$ lineshapes. The next sec- 
tion describes three of the methods used to obtain a pure-absorptive mode lineshape in

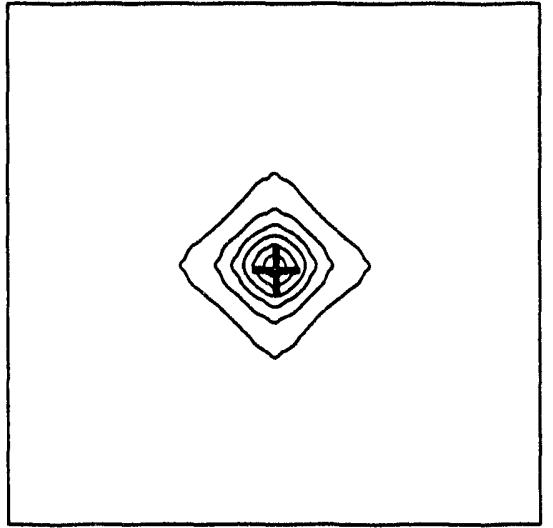

(a)

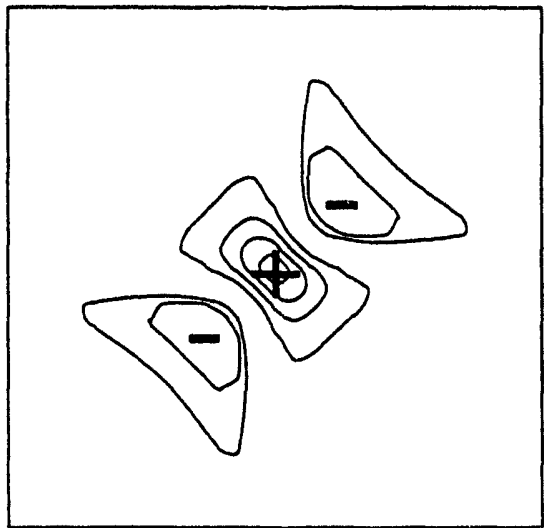

(b)

Figure 4.1 Pure-Absorption mode and Mixed-Phase 2D NMR Spectra. Spectrum (a) is an example of a pure-absorption mode line with equal homogeneous broadening in both dimensions. Spectrum (b) is an example of a mixed-phase line with the same parameters. Note in (b) the presence of both positive $(+)$ and $(-)$ contours, giving a peak of much larger effective linewidth.

2D NMR experiments. Each will be described briefly and in all cases additional information may be found in the papers referenced therein and in the classic text on multi-dimensional NMR by Ernst et al. ${ }^{7}$

\section{Pure-Absorption Mode Acquisition Methods}

The three most important methods for achieving pure-absorption phase multi-dimensional NMR data, States, TPPI and whole echo acquisition, are described in moderate detail in the next section. Each section contains references to other more complete descriptions of these experiments and the interested reader is directed there for additional information.

\section{States Method}

The method developed by States $e t$ al. ${ }^{88}$ creates pure-absorption mode spectra by acquiring a hypercomplex data set. In this type of data acquisition, there are two parts, a 
cosine labeled $S_{c}\left(t_{1}, t_{2}\right)$ and a sine labeled $S_{s}\left(t_{1}, t_{2}\right)$ portion. Thus, twice as many data points are needed to get the same resolution as a comparable phase-modulated experiment. The hypercomplex data is collected such that the cosine portion is quadrature in $t_{2}$ and amplitude modulated by $\cos \left(\Omega_{1} t_{1}\right)$ in $t_{1}$ while the sine portion is amplitude modulated by $\sin \left(\Omega_{1} t_{1}\right)$ in $t_{1}$. Mathematically, the two signals are expressed in equation 4.5 .

$$
\begin{aligned}
& S_{c}\left(t_{1}, t_{2}\right)=\cos \left(\Omega_{1} t_{1}\right) e^{-T_{2}^{-1}\left(t_{1}+t_{2}\right)} e^{-i \Omega_{2} t_{2}} \\
& S_{s}\left(t_{1}, t_{2}\right)=\sin \left(\Omega_{1} t_{1}\right) e^{-T_{2}^{-1}\left(t_{1}+t_{2}\right)} e^{-i \Omega_{2} t_{2}}
\end{aligned}
$$

To process this type of data, the $t_{2}$ Fourier transform is performed separately on each data set. This yields two new signal functions $S_{c}\left(t_{1}, \omega_{2}\right)$ and $S_{s}\left(t_{1}, \omega_{2}\right)$ given below.

$$
\begin{aligned}
& S_{c}\left(t_{1}, \omega_{2}\right)=\cos \left(\Omega_{1} t_{1}\right) e^{-T_{2}^{-1} t_{1}}\left(A\left(\omega_{2}, \Omega_{2}\right)+i D\left(\omega_{2}, \Omega_{2}\right)\right) \\
& S_{s}\left(t_{1}, \omega_{2}\right)=\sin \left(\Omega_{1} t_{1}\right) e^{-T_{2}^{-1} t_{1}}\left(A\left(\omega_{2}, \Omega_{2}\right)+i D\left(\omega_{2}, \Omega_{2}\right)\right)
\end{aligned}
$$

These are then combined to form a data set $S_{p p}\left(t_{1}, \omega_{2}\right)$ whose real components are the ortion of $S_{c}\left(t_{1}, \omega_{2}\right)$ and whose imaginary components are the negated real portion of $S_{s}\left(t_{1}, \omega_{2}\right)$.

$$
\begin{aligned}
S_{p p}\left(t_{1}, \omega_{2}\right) & =A\left(\omega_{2}, \Omega_{2}\right) e^{-T_{2}^{-1} t_{1}}\left(\cos \left(\Omega_{1} t_{1}\right)-i \sin \left(\Omega_{1} t_{1}\right)\right) \\
& =A\left(\omega_{2}, \Omega_{2}\right) e^{-T_{2}^{-1} t_{1}} e^{-i \Omega_{1} t_{1}}
\end{aligned}
$$

This data set is now ready to be Fourier transformed with respect to $t_{1}$. Notice that there is no dispersive $D\left(\omega_{2}, \Omega_{2}\right)$ term in the $S_{p p}\left(t_{1}, \omega_{2}\right)$ expression. In fact the final $S_{p p}\left(\omega_{1}, \omega_{2}\right)$ will have no dispersive contribution to the real channel (which is what normally is displayed).

$$
S_{p p}\left(\omega_{1}, \omega_{2}\right)=A\left(\omega_{1}, \Omega_{1}\right) A\left(\omega_{2}, \Omega_{2}\right)+i D\left(\omega_{1}, \Omega_{1}\right) A\left(\omega_{2}, \Omega_{2}\right)
$$

This will yield a truly puri-absorption mode lineshape, such as in figure 4.1. To implement a phase cycle to collect this type of hypercomplex data set, the data must be collected with both the +1 and -1 coherence pathways in $t_{1}$. When summed together they yield a cosine pathway and when subtracted they yield a sine pathway. A simple method 
for deriving a phase cycle for a hypercomplex data set is to take a phase cycle which chooses only the -1 or +1 pathway in $t_{1}$ and split this cycle into two phase cycles. One will be made up of those cycles which generate cosine in $t_{1}$ and the other will have those which generate sine in $t_{1}$. This is a little more difficult when $t_{1}$ is split between two different evolution periods (such as the original DAS experiment), but can still be accomplished with proper phase cycling and pulses (see Mueller et al. ${ }^{50}$ )

\section{Time Proportional Phase Incrementation}

The technique of time proportional phase incrementation (TPPI) ${ }^{89,90}$ is mathematically equivalent to the method derived by States. Again, twice as many data points must be collected as in a phase-modulated experiment with the same resolution. The basic difference between States method and TPPI arises in the data acquisition and processing. To acquire TPPI data, a $S_{\text {TPPI }}\left(t_{1}, t_{2}\right)$ data set is collected where the dwell time $\Delta t_{1}$ is one half and the number of $t_{1}$ points is twice what would be normally used in a phase modulated experiment, giving both the same spectral width and digital resolution in $t_{1}$. In addition, all the pulses immediately before the $t_{1}$ evolution period begins are incremented by $90^{\circ}$ after each $t_{1}$ point.

$$
S_{T P P I}\left(t_{1}, t_{2}\right)=e^{-T_{2}^{-1}\left(t_{1}+t_{2}\right)} \cos \left(\Omega_{1} t_{1}+\frac{\pi t_{1}}{2 \Delta t_{1}}\right) e^{i \Omega_{2} t_{2}}
$$

This data is Fourier transformed with respect to $t_{2}$ exactly as a usual phase modulated data set to yield $S_{T P P I}\left(t_{1}, \omega_{2}\right)$.

$$
\begin{aligned}
S_{T P P I}\left(t_{1}, \omega_{2}\right) & =e^{-T_{2}^{-1} t_{1}} \cos \left(\left(\Omega_{1}+\frac{\pi}{2 \Delta t_{1}}\right) t_{1}\right)\left(A\left(\omega_{2}, \Omega_{2}\right)+i D\left(\omega_{2}, \Omega_{2}\right)\right) \\
& =e^{-T_{2}^{-1} t_{1}}\left(\begin{array}{l}
\left.A\left(\omega_{2}, \Omega_{2}\right) \cos \left(\left(\Omega_{1}+\frac{\pi}{2 \Delta t_{1}}\right) t_{1}\right)+\right) \\
i D\left(\omega_{2}, \Omega_{2}\right) \cos \left(\left(\Omega_{1}+\frac{\pi}{2 \Delta t_{1}}\right) t_{1}\right)
\end{array}\right)
\end{aligned}
$$

The imagirary portion of this data set is then thrown out and the remaining real portion is Fourier transformed with a real Fourier transform in $t_{1}$ (rather than the usual complex 
Fourier transform). This type of Fourier transform has no quadrature and peaks show up at both the positive and negative frequencies. This is where the phase incrementation "trick" can be seen. Each term has a $\omega_{1}$ frequency of $\Omega_{1}+\frac{\pi}{2 \Delta t_{1}}$ rather than just $\Omega_{1}$. Therefore the signal will be given by equation 4.11 .

$$
S_{T P P I}\left(\omega_{1}, \omega_{2}\right)=\left(\begin{array}{l}
A\left(\omega_{2}, \Omega_{2}\right)\left(A\left(\omega_{1}, \Omega_{1}+\frac{\pi}{2} \cdot \frac{\pi}{t_{1}}\right)+A\left(\omega_{1},-\Omega_{1}-\frac{\pi}{2 \Delta t_{1}}\right)\right)- \\
i A\left(\omega_{2}, \Omega_{2}\right)\left(D\left(\omega_{1}, \Omega_{1}+\frac{\pi}{2 \Delta t_{1}}\right)+D\left(\omega_{1},-\Omega_{1}-\frac{\pi}{2 \Delta t_{1}}\right)\right)
\end{array}\right)
$$

The resulting spectrum is symmetric about zero frequency and the negative side may now be thrown out. The remaining positive frequency data set is pure-absorption mode and may be made equivalent to the States result by setting the center of the spectrum to zero frequency (a shift of $\frac{\pi}{2 \Delta t_{1}}$ ). The phase cycle for this type of spectrum is identical to the phase cycle for the cosine portion of the hypercomplex data set from the previous section with the addition of the time proportional phase incrementation of the pulses before the start of $t_{1}$.

\section{Whole Echo Acquisition}

Whole echo acquisition ${ }^{91}$ has been less popular than the other methods of obtaining pure phase spectra. This is primarily because in the case of liquid spectra, it is difficult to obtain whole echoes in $t_{2}$ since the lines are so narrow. In fact, when only a fraction of the echo is collected phase twist components will enter into the final 2D spectrum. In the case of solids, where the inhomogeneous broadening is usually much larger than the homogeneous broadening, whole echo acquisition can actually be better than the other methods. To understand why two-dimensional whole echo acquisition works, first it is useful to look at a one-dimensional case. Suppose you generate a Gaussian-shaped timedomain echo with a $90^{\circ}-180^{\circ}\left(\pi / 2-t_{e}-\pi-a c q\right)$ sequence which has a signal $S_{e}\left(t_{2}\right)$ given by equation 4.12 . 


$$
S_{e}\left(t_{2}\right)=e^{-T_{2}^{-2}\left(t_{2}-t_{e}\right)^{2}} e^{-i \Omega_{2}\left(t_{2}-t_{e}\right)}
$$

When this signal is Fourier transformed, a spectrum of the form given by equation 4.13 will be generated, assuming that the signal is shifted far enough out in time and is zero at both the first and last $t_{2}$ points.

$$
S_{e}\left(\omega_{2}\right)=e^{-\frac{\left(\omega_{2}-\Omega_{2}\right)^{2} T_{2}^{2}}{4}} e^{i \omega_{e}}=A_{e}\left(\omega_{2}, \Omega_{2}\right)
$$

This appears at first glance to be much worse than if we had only collected from the echo top on, due to the phase factor. However, by effectively shifting the time origin by applying a first-order phase correction of $t_{e}$ (which multiplies each point in the spectrum by $\left.e^{-i \omega x_{e}}\right)$, the spectrum is greatly simplified.

$$
S_{s e}\left(\omega_{2}\right)=e^{-\frac{\left(\omega_{2}-\Omega_{2}\right)^{2} T_{2}^{2}}{4}}=A_{s e}\left(\omega_{2}, \Omega_{2}\right)
$$

This shifted-echo (hence the se subscript) spectrum has no dispersive imaginary components. This can be quite useful in a two-dimensional experiment where the signal is of the form.

$$
S_{e}\left(t_{1}, t_{2}\right)=e^{-T_{2}^{-2}\left(t_{2}-t_{e}\right)^{2}} e^{-i \Omega_{2}\left(t_{2}-t_{e}\right)} e^{-T_{2}^{-1} t_{1}} e^{-i \Omega_{1} t_{1}}
$$

This is a constant time echo experiment and the first Fourier transform is done as usual in the $t_{2}$ dimension. The resulting signal function has the form given in equation 4.16.

$$
S_{e}\left(t_{1}, \omega_{2}\right)=A_{e}\left(\omega_{2}, \Omega_{2}\right) e^{-T_{2}^{-t_{1}} e^{-i \Omega_{1} t_{1}}}
$$

A first order phase correction of $t_{e}$ is then applied to the $\omega_{2}$ dimension which yields signal with the function given in equation 4.17 (note the se subscript on the absorptive onedimensional $A_{s e}\left(\omega_{2}, \Omega_{2}\right)$ function).

$$
S_{s e}\left(t_{1}, \omega_{2}\right)=A_{s e}\left(\omega_{2}, \Omega_{2}\right) e^{-T_{2}^{-1} t_{1}} e^{-i \Omega_{1} t_{1}}
$$

A Fourier transform is then applied to the $t_{1}$ dimer-ion giving a pure-absorption mode 2D spectrum as the result (see equation 4.18). 


$$
S_{e}\left(\omega_{1}, \omega_{2}\right)=A_{s e}\left(\omega_{2}, \Omega_{2}\right)\left(A\left(\omega_{1}, \Omega_{1}\right)+i D\left(\omega_{1}, \Omega_{1}\right)\right)
$$

This result is particularly important because data of this sort doesn't require the factor of 2 additional $t_{1}$ points like the States or TPPI methods, thus it will have a $\sqrt{2}$ improvement in signal-to-noise over States or TPPI data. However, this is only true in situations where the entire echo may be collected for every $t_{1}$ point, which in general will only be the case for solids with a strong inhomogeneous broadening. The phase cycle necessary to collect a whole echo is not any different than collecting a standard phase-modulated data set. In fact, in some cases TPPI or States methods may be applied in concert with whole echo acquisition to gain an additional $\sqrt{2}$ improvement in signal-to-noise ratio. ${ }^{53}$

\section{Pure Phase DAS}

The original DAS experiment as described by both Mueller et al. ${ }^{42}$ and Llor and Virlet ${ }^{43}$ was a phase modulated experiment (with the phase cycles given in the papers) and gave phase-twist lineshapes in two dimensional spectra which necessitated magnitude mode display (see figire 3.6). To obtain higher resolution, pure-phase two dimensional DAS experiments were first developed by Mueller et al. ${ }^{42,47}$ In this work, they viewed the DAS experiment in a non-sheared fashion and used either a $\mathrm{z}$-filter or a $90^{\circ}$ pulse after the total $t_{1}$ evolution period to give pure-absorption mode spectra. As will be discussed below, we have been able to obtain higher signal to noise ratio pure-absorption mode DAS spectra by redefining our time axes. ${ }^{53}$ Also, further sensitivity improvements have been made by shifting the DAS echo in time using $\pi$ pulses.

\section{Pulse Sequences}

The original DAS pulse sequence has been discussed earlier but will be reviewed to show the differences between it and the new pulse sequences. In figure 4.2 we see a simulated DAS spectrum acquired with the original DAS sequence. The mixed-phase artifacts are seen in the upper right and lower left side of the spectrum as broad negative re- 
gions. This overall phase twist will make the slices through the isotropic peaks difficult to interpret.

(a)

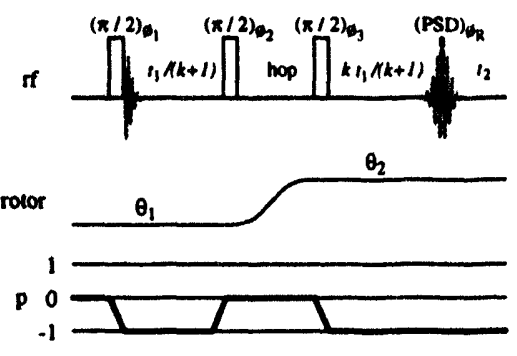

(b)

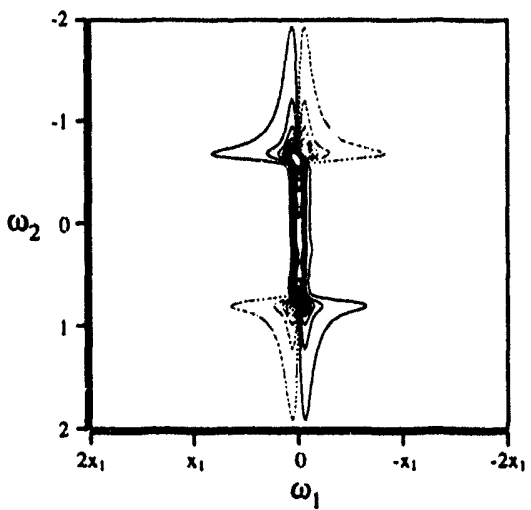

Figure 4.2 Original DAS Experiment. The pulse sequence and coherence pathway (a) is shown to the left of the simulated spectrum (b). The phase cycle used to implement this pathway was given earlier. Dashed contour lines indicate negative contours.

Figure 4.3 shows the modified DAS experiment where the time axes have been redefined, very similar to the time definitions in a $2 \mathrm{D}$ exchange experiment (and identical to those definitions discussed in the description of spinning sidebands in chapter 3 ). In this experiment, the evolution at the first angle is defined as $t_{1}$ and the evolution after the hop is defined as $t_{2}$. This definition will place a shifting DAS isotropic echo in the $t_{2}$ dimension. In fact, this echo will appear at a time $k t_{1}$. When this data is processed without modification, we observe a diagonal peak which is the correlation between anisotropic patterns in both dimensions. A conventional 2D DAS spectrum may be obtain:d by shearing this spectrum by an angle $\theta_{s}$ (as was mentioned earlier in the spinning sidebands section of chapter 3 ).

$$
\theta_{s}=\tan ^{-1} k
$$

Another method for shearing the ${ }^{\prime}$ ata is to apply a $t_{1}$ dependent first-order phase correction of $\phi\left(t_{1}, \omega_{2}\right)$ to the data set between the first and second Fourier transforms.

$$
\begin{aligned}
\phi\left(t_{1}, \omega_{2}\right) & =k \omega_{2} t_{1} \\
S^{\prime}\left(t_{1}^{\prime}, \omega_{2}^{\prime}\right) & =e^{-i \phi\left(t_{1}, \omega_{2}\right)} S\left(t_{1}, \omega_{2}\right)
\end{aligned}
$$




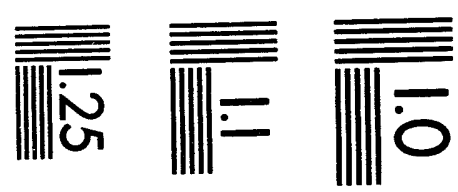

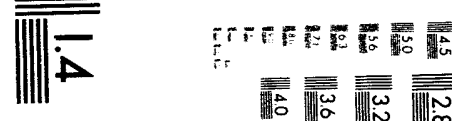

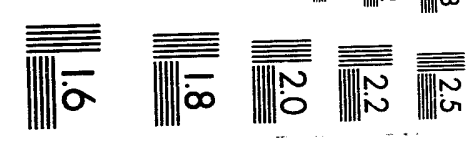



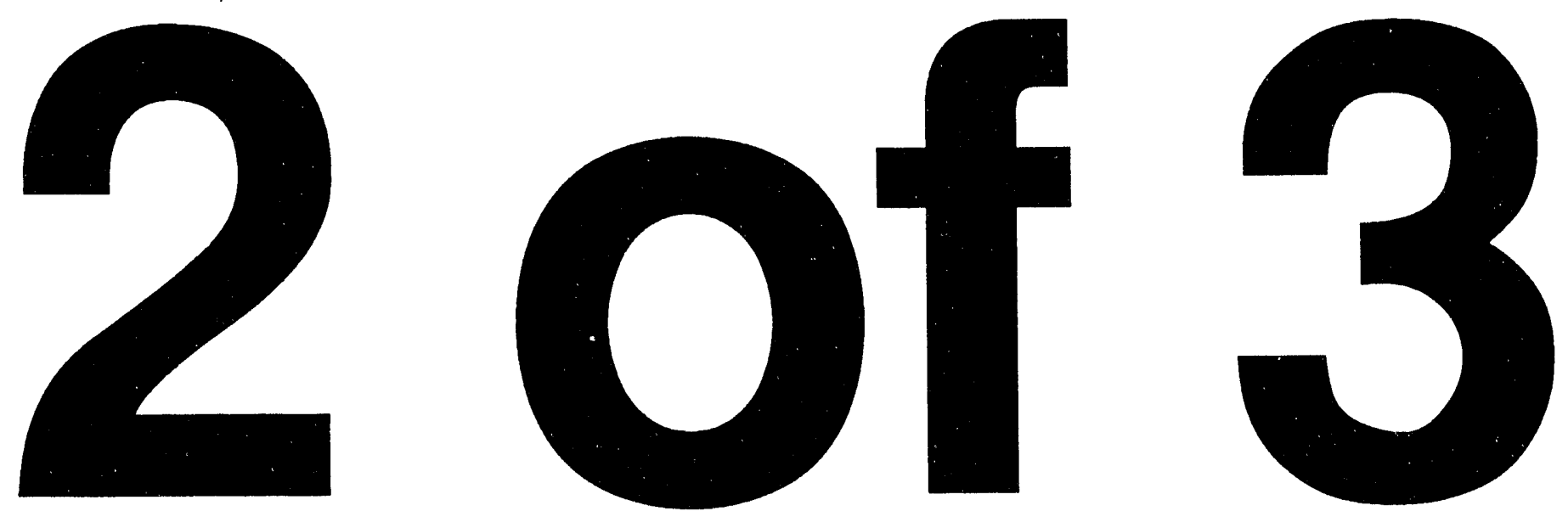
This method of acquisition produces a phase-twist spectrum, but because an entire echo is collected for the later $t_{1}$ points, the negative contours are much smaller, giving effectively higher resolution in both dimensions.

(a)

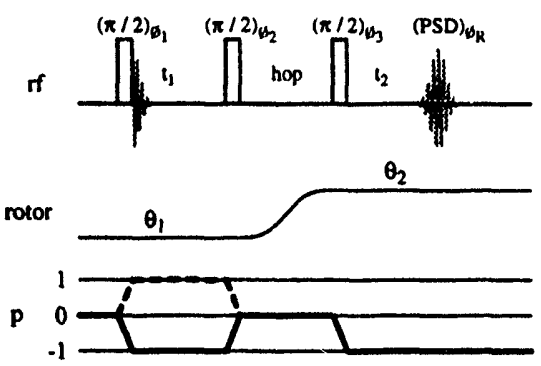

(b)

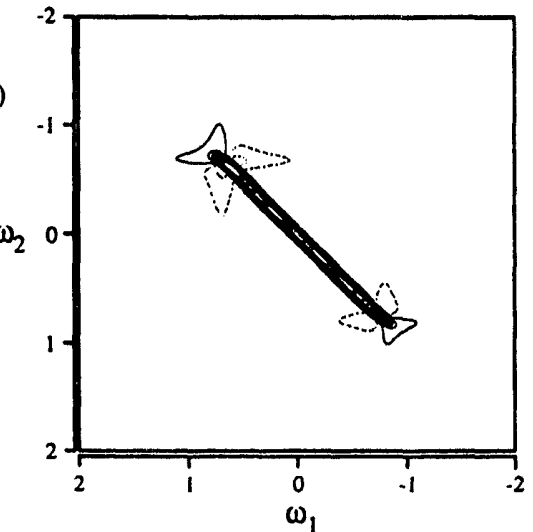

Figure 4.3 Modified DAS Experiment. The (a) pulse sequence and coherence pathway are shown to the left of $(b)$ the unsheared simulated spectrum. The dashed line coherence pathway indicates the anti-echo DAS experiment. The phase cycle used to implement this pathway is identical to the original DAS experiment. Dashed contour lines indicate negative contours.

A second modification to the DAS experiment may be made by using either the method of States et al ${ }^{88}$ or TPPI ${ }^{89,90}$ to acquire pure-absorption mode spectra using the same $t_{1}$ and $t_{2}$ definitions. To accomplish this, we need to merely change the way the data is collected. Rather than collecting a single data set as a function of $t_{1}$ and $t_{2}$, we collect a hypercomplex data set as a function of $t_{1}$ and $t_{2}$. As mentioned previously, a hypercomplex data set separates the sine and cosine evolution in $t_{1}$. Each of these data sets is Fourier transformed with respect to $t_{2}$. This produces a data set with absorptive lineshapes in the real channel and dispersive lineshapes in the imaginary channel. The real portion of the cosine data set is combined with $\sqrt{-1}$ times the real portion of the sine data set. Thus there are only absorptive lineshapes in $\omega_{2}$ which are modulated by $e^{-i \Omega_{1} t_{1}}$ in the $t_{1}$ dimension. Applying the same $t_{1}$ dependent first-order phase correction from equation 4.2, we then can perform the $t_{1}$ Fourier transform. This yields pure-absorption mode 2D DAS spectra. There are no mixed-phase artifacts to make interpretation difficult. The phase cycle and coherence pathway for this experiment are given in figure 4.4. 
(a)
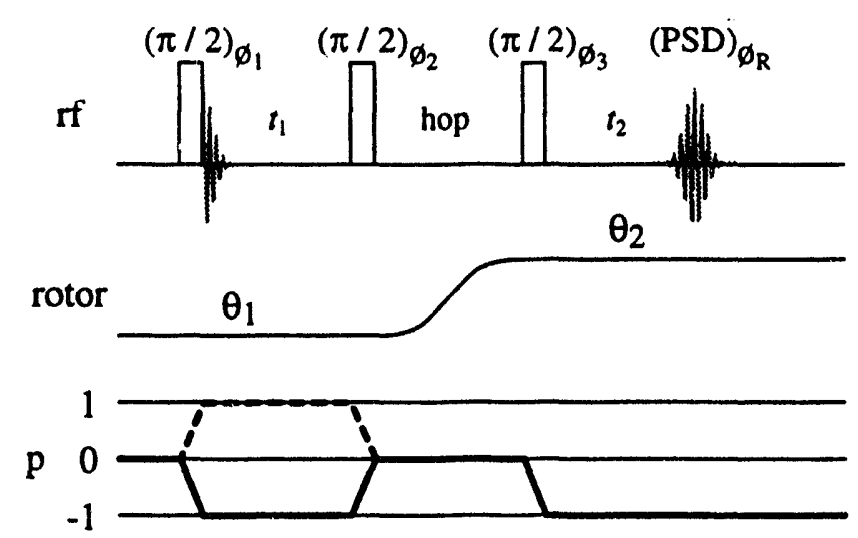

(b)

\begin{tabular}{llll}
$\emptyset_{1}$ & $\emptyset_{2}$ & $\emptyset_{3}$ & $\emptyset_{\mathrm{R}}$ \\
\hline 0 & 0 & 0 & 0 \\
180 & 0 & 0 & 180 \\
0 & 180 & 0 & 0 \\
180 & 180 & 0 & 180
\end{tabular}

(c)

\begin{tabular}{llll}
$\emptyset_{1}$ & $\emptyset_{2}$ & $\emptyset_{3}$ & $\emptyset_{\mathrm{R}}$ \\
\hline 90 & 0 & 0 & 0 \\
270 & 0 & 0 & 180 \\
90 & 180 & 0 & 0 \\
270 & 180 & 0 & 180
\end{tabular}

Figure 4.4 Hypercomplex DAS Experiment. The pulse sequence (a), coherence pathway, and phase cycle are given above. Cycle (b) is the cosine data set and cycle (c) is the sine data set.

An alternative method of sensitivity improvement in dynamic-angle spinning experiments comes from shifting the isotropic DAS echo in $t_{2}$. This is accomplished by applying a $\pi$ pulse after a $n t_{r}$ delay following the final $\pi / 2$ read pulse. This shifted echo DAS (SEDAS) pulse sequence is detailed in figure 4.5. This sequence has the advantage of shifting the DAS echoes in time by $n t_{r}$. For all $t_{1}$ values, an entire DAS echo may be collected which leads to a higher signal-to-noise ratio than the hypercomplex DAS which takes twice as long to effectively collect whole echoes in $t_{1}$. This is especially important in cases where the broadening is primarily inhomogeneous and anisotropic in the $\omega_{2} \mathrm{di}-$ mension. 
(a)
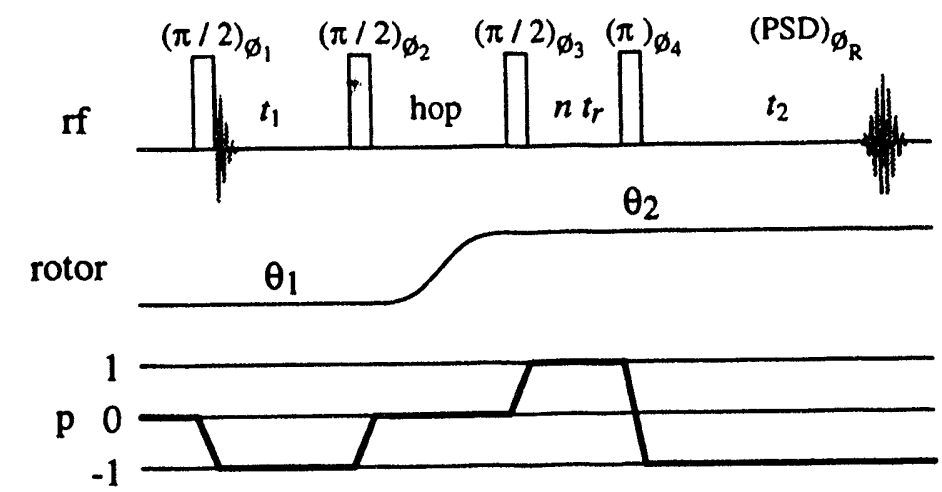

(b) \begin{tabular}{lllll}
$\emptyset_{1}$ & $\emptyset_{2}$ & $\emptyset_{3}$ & $\emptyset_{4}$ & $\emptyset_{\mathrm{R}}$ \\
\hline 0 & 0 & 0 & 0 & 0 \\
90 & 0 & 0 & 0 & 90 \\
180 & 0 & 0 & 0 & 180 \\
270 & 0 & 0 & 0 & 270 \\
0 & 0 & 90 & 0 & 270 \\
90 & 0 & 90 & 0 & 0 \\
180 & 0 & 90 & 0 & 90 \\
270 & 0 & 90 & 0 & 180 \\
0 & 0 & 180 & 0 & 180 \\
90 & 0 & 180 & 0 & 270 \\
180 & 0 & 180 & 0 & 0 \\
270 & 0 & 180 & 0 & 90 \\
0 & 0 & 270 & 0 & 90 \\
90 & 0 & 270 & 0 & 180 \\
180 & 0 & 270 & 0 & 270 \\
270 & 0 & 270 & 0 & 0
\end{tabular}

Figure 4.5 Shifted-Echo DAS Experiment. The pulse sequence (a), coherence pathway, and phase cycle (b) are given above.

In cases where the broadening is inhomogeneous in both the $\omega_{2}$ and $\omega_{1}$ dimensions of a DAS experiment, further advantage may be had by collecting hypercomplex data in concert with a shifted echo experiment. This hypercomplex SEDAS experiment is shown schematically in figure 4.6. The phase cycle for both the cosine and sine portions of the data set are indicated as well. In both the SEDAS and HyperSEDAS experiments, both the first and third pulses are phase cycled through four steps each. This effectively chooses only a -1 (or both +1 and -1 in the hypercomplex division of the phase cycle in figure 4.6) coherence after the first pulse and guarantees a +1 coherence following the third pulse. This sequence effectively collects both the echo and anti-echo DAS 
signals. In the case of a crystalline sample, the anti-echo signal will shift to the left in $t_{2}$ as $t_{1}$ increases while the echo signal will shift to the right in $t_{2}$ as $t_{1}$ increases.

(a)
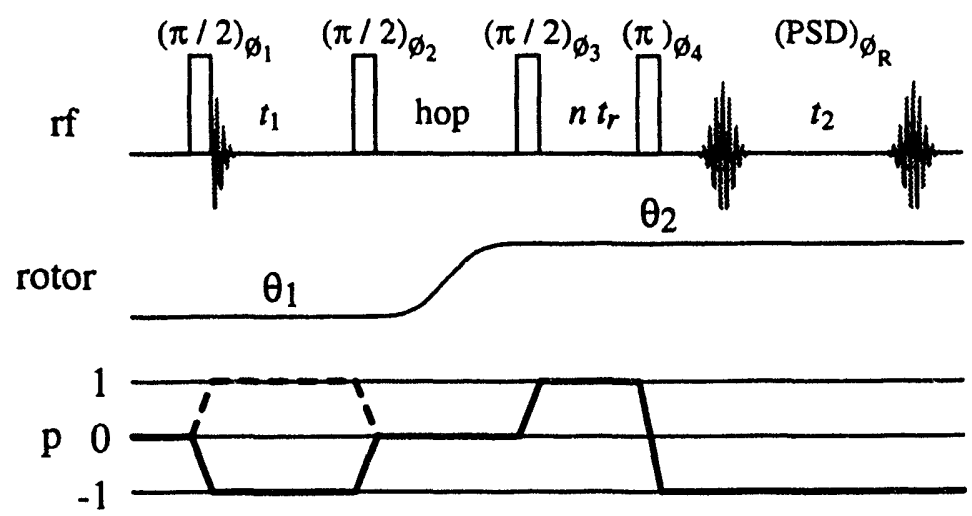

(b)

\begin{tabular}{lllll}
$\emptyset_{1}$ & $\emptyset_{2}$ & $\emptyset_{3}$ & $\emptyset_{4}$ & $\emptyset_{\mathrm{R}}$ \\
\hline 0 & 0 & 0 & 0 & 0 \\
180 & 0 & 0 & 0 & 180 \\
0 & 0 & 90 & 0 & 270 \\
180 & 0 & 90 & 0 & 90 \\
0 & 0 & 180 & 0 & 180 \\
180 & 0 & 180 & 0 & 0 \\
0 & 0 & 270 & 0 & 90 \\
180 & 0 & 270 & 0 & 270
\end{tabular}

(c)

\begin{tabular}{lllll}
$\emptyset_{1}$ & $\emptyset_{2}$ & $\emptyset_{3}$ & $\emptyset_{4}$ & $\emptyset_{\mathrm{R}}$ \\
\hline 90 & 0 & 0 & 0 & 0 \\
270 & 0 & 0 & 0 & 180 \\
90 & 0 & 90 & 0 & 270 \\
270 & 0 & 90 & 0 & 90 \\
90 & 0 & 180 & 0 & 180 \\
270 & 0 & 180 & 0 & 0 \\
90 & 0 & 270 & 0 & 90 \\
270 & 0 & 270 & 0 & 270
\end{tabular}

Figure 4.6 Hypercomplex Shifted-Echo DAS Experiment. The pulse sequence (a), coherence pathway, and phase cycle for the cosine data set (b) and sine data set (c) are given above. Two echos are shown in the above figure since the signal will have an echo contribution from both the echo (solid line) and anti-echo (dashed line) pathways which may not necessarily occur at the same point.

In this case, the anti-echo will often shift out of the window before decaying to zero intensity in $t_{1}$. In most cases (assuming enough points are taken in $t_{2}$ ) the echo signal will always remain in the observation window. If the $n t_{r}$ decay is chosen long enough so that 
both the echo and anti-echo data remain in the acquisition window for all $t_{1}$ points, the total intensity will be nearly zero (in analogy to collecting long delay times in a constant time experiment).

In the case of a sample with a broad inhomogeneous distribution of sites in both dimensions, the echo will decay away much faster in $t_{1}$. In addition, if the distribution in $t_{1}$ is continuous, the anti-echo will not shift to the left in $t_{2}$ as rapidly in $t_{1}$. Likewise, the echo will not shift to the right in $t_{2}$ as quickly in $t_{1}$. For the case of an amorphous solid, the hypercomplex SEDAS is the best pulse sequence, since it combines the signal-tonoise enhancements of an echo in $t_{2}$ with hypercomplex data in $t_{1}$. Chapter 8 gives specific examples of glasses with distributions of isotropic shift, for which acquisition with HyperSEDAS gave significant improvements in sensitivity.

\section{Experimental Examples}

The following figures (figs. 4.7, 4.8, and 4.9) show examples of various types of DAS spectra. All of these spectra were taken with $k=5$ (the angle pairs were $63.43^{\circ}$ and $0.00^{\circ}$ ) and the magnetic field strength was $11.7 \mathrm{~T}$. The sample used was a standard reagent grade $\mathrm{RbClO}_{4}$ sample. The pulse sequences for each experiment are indicated in each figure caption. The $90^{\circ}$ and $180^{\circ}$ selective pulses were $3.35 \mu \mathrm{s}$ and $6.70 \mu \mathrm{s}$ respectively, the axis reorientation time was $50 \mathrm{~ms}$, the spinning rate was $5.8 \mathrm{kHz}$, the echo time in SEDAS and HyperSEDAS experiments was $1.029 \mathrm{~ms}$ and the number of acquisitions for each $t_{1}$ point was 128 scans for both the sine and cosine data sets. The hypercomplex sine and cosine data sets were combined to produce the normal phase modulated data in $t_{1}$. The dwell time in the $t_{2}$ dimension and in the $t_{1}$ dimension following proper shearing was $50 \mu$ s for both. The acquisition length in the second dimension was 256 complex points while it was 128 points in the first dimension.

Figure 4.7 shows the normal phase modulated DAS data set acquired with the pulse sequence in figure 4.2 showing the usual phase-twist lineshape. The phase-twist 
lineshape greatly increases the effective linewidth in the anisotropic dimension. In this spectrum (and in figs. 4.8 and 4.9), contours were placed at $-12,14,41,68$ and 95 percent of the maximum. The negative contours appear to the lower left and upper right of the center of the main peak, just as they occur in figs $4.1 \mathrm{~b}$ and 4.2 .

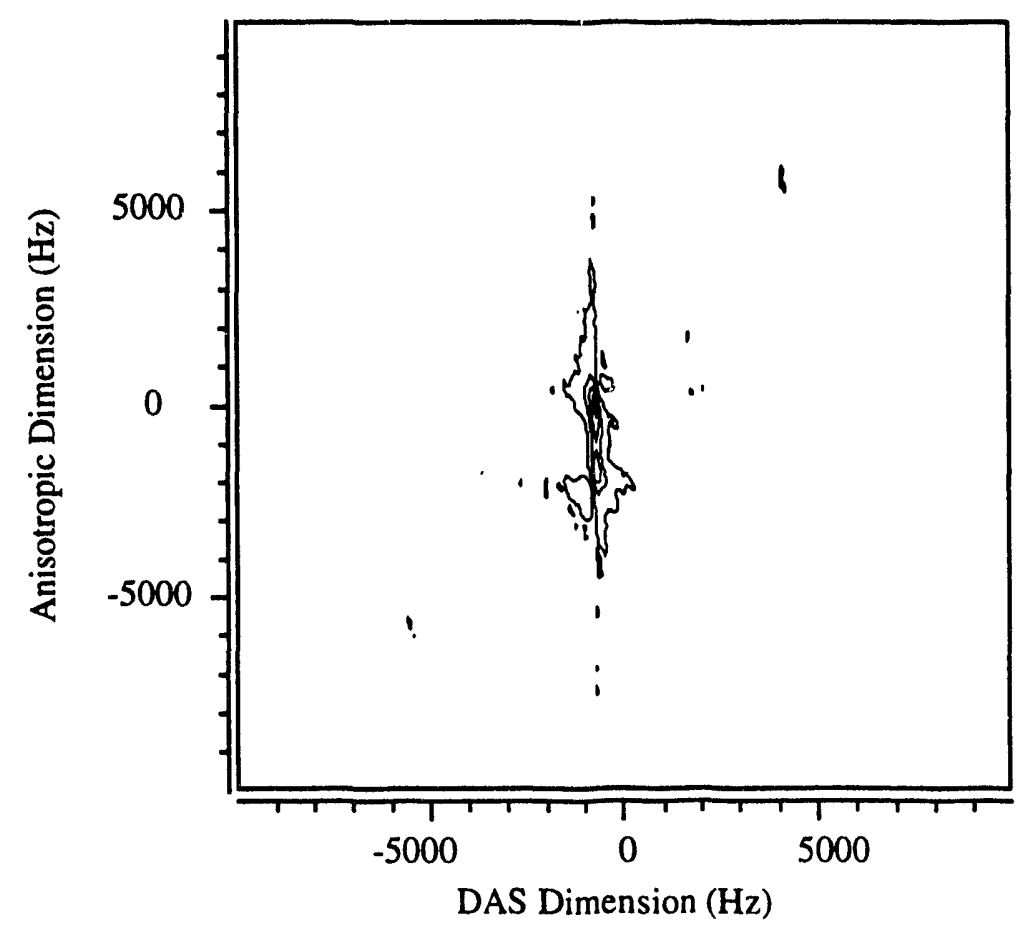

Figure 4.7 Original DAS Spectrum. This spectrum was taken with the parameters given in the text above and with the pulse sequence in figure 4.2 .

Figure 4.8 shows the echo DAS spectrum collected with the pulse sequence in figure 4.3. Note that a $41.67 \mu \mathrm{s} t_{1}$ dependent first-order phase correction was required to shear the two-dimensional spectrum. This spectrum is not quite completely pure-absorptive phase. However, the dispersive contributions are of small enough size that they do not change the overall appearance of this spectrum in reference to the completely pureabsorptive phase spectrum in figure 4.9 .

Figure 4.9 shows the pure-absorptive spectrum acquired with the hypercomplex DAS pulse sequence (figure 4.4). The spectra acquired with SEDAS and HyperSEDAS look virtually identical and are not shown. The lineshape shows no phase-twist dispersive components in the two-dimensional spectrum above. 


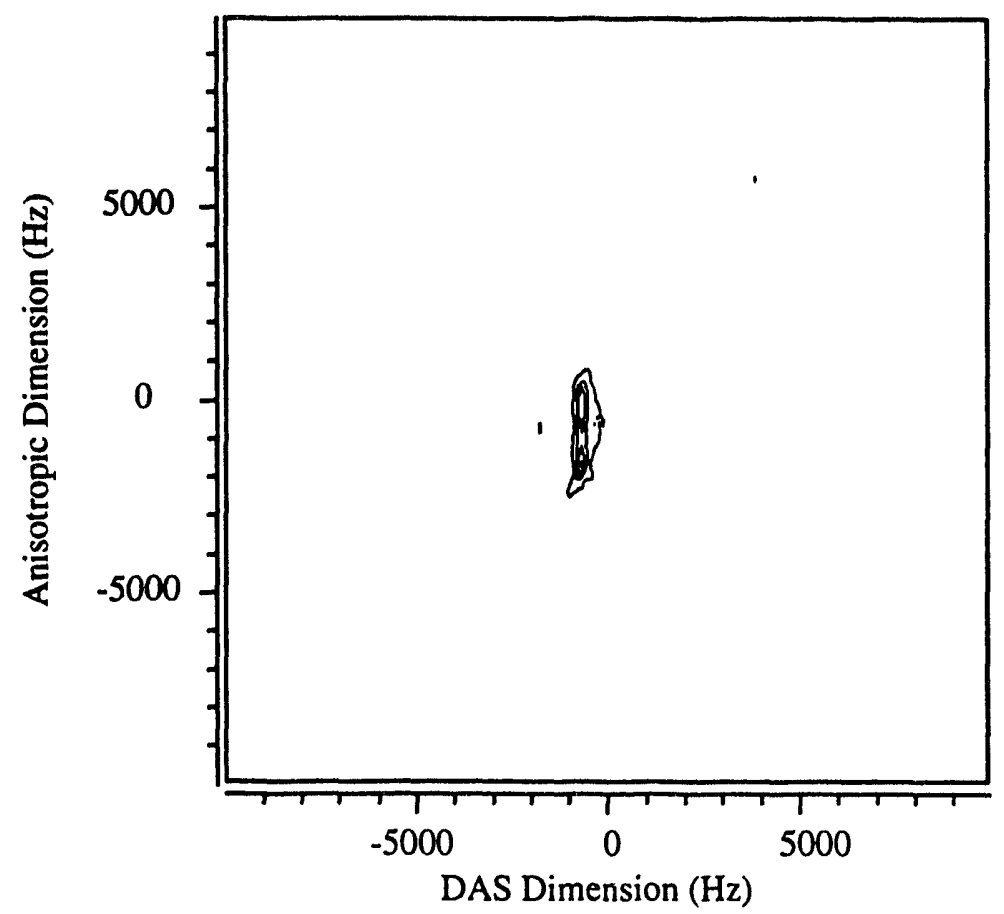

Figure 4.8 Echo DAS Spectrum. This spectrum was taken with the pulse sequence given in figure 4.3.

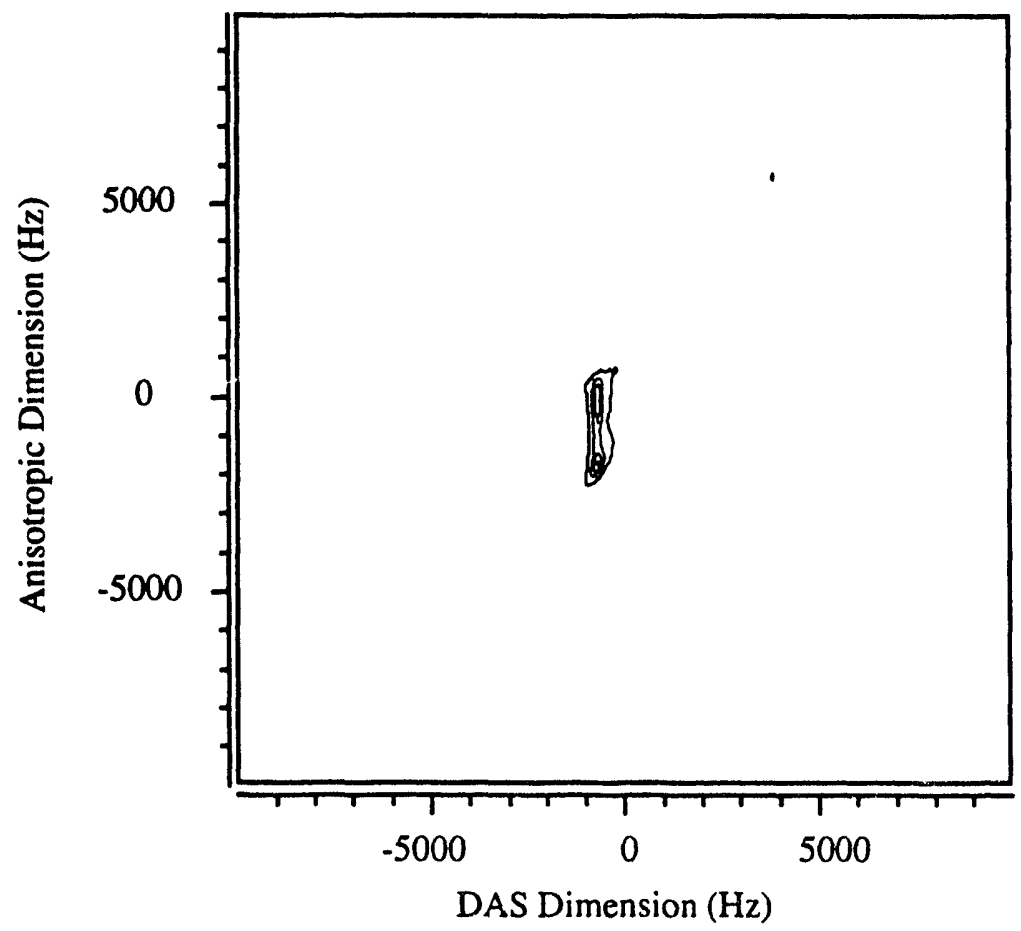

Figure 4.9 Hypercomplex DAS Spectrum. This spectrum was taken with the pulse sequence given in figure 4.3. 


\section{Signal-to-Noise Ratio Enhancements}

In the case of one-dimensional DAS spectra, the signal-to-noise enhancement will be independent of the enhancement in the anisotropic dimension. In practice, the shifted echo experiments will provide better signal-to-noise, since the complete DAS echo signal can be collected for short $t_{1}$ points. For short $t_{1}$ points in the non-shifted DAS experiments, the echo top intensity may be complicated by ringing of the probe. This ringing can significantly reduce the signal-to-noise ratio in the one-dimensional projections. Also, by doing only a partial projection of the signal in the two dimensional spectrum (rather than a complete projection) by adding only regions with strong signal, significant improvements in signal-to-noise ratio in the one dimensional DAS spectra may be achieved. This may distort the overall intensities in the final DAS spectrum and in some cases it is not possible to eliminate any region of the two dimensional spectrum for projection.

In table 4.1, the two dimensional signal-to-noise ratios are tabulated for each of the various DAS pulse sequences. These numbers are arrived at by measuring the RMS noise in a region of the 2D spectrum which is devoid of signal and comparing this to the highest point (largest signal) in the complete 2D spectrum. The experimental examples shown in the previous section were used to generate these ratios. As has been predicted by theory, the hypercomplex SEDAS experiment has the highest signal-to-noise ratio. This should in theory be a factor of $\sqrt{2} \approx 1.4$ better than the SEDAS experiment. In this case, the factor was indeed achieved, but in practice this may not be always be true, since the DAS anti-echo may shift out of the acquisition window too rapidly. The SEDAS signal-to-noise ratio should also be a factor of $\sqrt{2} \approx 1.4$ better than both the echo DAS and hypercomplex DAS experiments. 


\begin{tabular}{lcc}
\hline \multicolumn{1}{c}{ Sequence } & Pure Phase & S/N Ratio \\
\hline z-Filter DAS & yes & 15.8 \\
DAS (fig. 4.2) & no & 20.6 \\
Hypercomplex DAS (fig. 4.4) & yes & 31.3 \\
Echo DAS (fig. 4.3) & no & 31.6 \\
Anti-echo DAS (fig 4.3, dashed pathway) & no & 10.9 \\
Shifted-echo DAS (fig. 4.5) & yes & 43.4 \\
Hypercomplex Shifted Echo (fig. 4.6) & yes & 67.0 \\
\hline
\end{tabular}

Table 4.1 Signal-to-Noise Ratio Enhancements For a Variety of Pulse Sequences. These measurements were all performed with equal acquisition time for each experiment.

Again, this enhancement seems to hold quite well. The hypercomplex DAS should be better by a factor of $\sqrt{2} \approx 1.4$ than the original DAS experiment, which also is true. Finally the z-filter pure phase method signal-to-noise ratio should be comparable to the original DAS experiment, since the z-filter sacrifices a factor of $\sqrt{2} \approx 1.4$ which is restored by the hypercomplex data collection. In practice, the $\mathrm{z}$-filter will have worse signal-to-noise ratios than the original phase modulated data, since relaxation during the $\mathrm{z}$ filter will further reduce the signal-to-noise in this type of experiment. Therefore, theory predicts that the signal to noise of the hypercomplex SEDAS will be at least a factor of $\sqrt{8} \approx 2.8$ better than the older $z$-filter method of acquiring pure-phase data. A final comment about pure-absorption phase DAS is to warn the reader that in some cases SEDAS or hypercomplex DAS may actually work better than the full hypercomplex SEDAS. This will occur when the apparent $T_{2}$ of a sample is too fast to allow long $n t_{\mathrm{r}}$ echo times. In most cases throughout this thesis, the SEDAS pulse sequence will be used. 


\section{Chapter 5}

\section{Cross Polarization}

The use of cross polarization in solid-state nuclear magnetic resonance has lead to the rapid advance of experiments on spin $1 / 2$ nuclei such as ${ }^{13} \mathrm{C}$ and ${ }^{15} \mathrm{~N}$. Both of these nuclei are very important in biological samples, especially peptides and proteins. The pioneering work with CPMAS by researchers such as Waugh and Griffin at MIT and Schaeffer at Washington University has opened the door for a variety of high resolution studies of these types of samples. A brief history of some of the theory behind cross polarization will be discussed below. Following that introduction, I will describe some of the difficulties in applying the technique of $\mathrm{CP}$ and CPMAS to quadrupolar nuclei and one solution to this problem.

\section{History}

The technique of cross polarization was first discovered over 30 years ago by Hartmann and Hahn..$^{92}$ This represents one of Hahn's many contributions to the field of magnetic resonance. In these experiments, magnetic polarization is transferred from one type of nuclear spin to another. This is accomplished by applying strong rf fields along the rotating frame $x$ axis to both types of spins following a $90^{\circ}$ excitation pulse along the y axis. In this case, the transverse magnetizations of both spins are "spin-locked" along the rotating frame $\mathrm{x}$ axis. In this rotating frame, the precession rate of each spin about the spinlocking magnetization will be determined by the respective strengths of the rf fields and gyromagnetic ratios. When the Hartmann-Hahn condition is achieved, the precession frequency of both types of spins will be equal, that is to say the rf amplitude is set to a level such that the $90^{\circ}$ pulse lengths are identical for both spin systems. Mathematically, this is expressed as $\gamma_{I} B_{1 I}=\gamma_{S} B_{1 S}$ for spin $1 / 2$ nuclei. This condition is quite sharp and appears much like other resonance phenomena. The reason that this allows polarization 
to be exchanged is that the flip-flop terms in the homonuclear dipolar Hamiltonian (those which mutually flip two different spins) are now zero energy processes. This greatly enhances the dipolar coupling and allows energy to be transferred between the two spin baths. The spin temperature of the two baths will rapidly come to equilibrium with the higher gyromagnetic ratio spins giving energy to the lower gyromagnetic ratio spins. This may be applied to systems such as ${ }^{1} \mathrm{H}-{ }^{13} \mathrm{C}$ to gain approximately a factor of $3-4$ in ${ }^{13} \mathrm{C}$ polarization or to ${ }^{1} \mathrm{H}-15 \mathrm{~N}$ to gain a factor of $9-10$ in ${ }^{15} \mathrm{~N}$ polarization. Additionally, since the ${ }^{1} \mathrm{H}$ bath is high abundance, the $T_{1}$ relaxation time will often be much faster than ${ }^{15} \mathrm{~N}$ or ${ }^{13} \mathrm{C}$ and the experimental repetition rate may be increased significantly 93 . Combining cross polarization with magic-angle spinning has the added advantage of giving high resolution spectra for nuclei like carbon-13 and nitrogen-15. ${ }^{94}$ In this respect, ${ }^{13} \mathrm{C}$ CPMAS has become a standard and routine experiment in most laboratories. In the next section, the use of cross polarization to study quadrupolar nuclei under VAS conditions will be discussed. Previously, CP has been applied to a number of different quadrupolar systems, with polarization usually being transferred from the abundant ${ }^{1} \mathrm{H}$ spins to the specific quadrupolar nucleus. ${ }^{28,60,65,73,95-106}$ I would direct the reader to these references for additional information on this subject.

\section{Spinning Effects on CP of Quadrupolar Nuclei}

Significant increases in NMR sensitivity can be achieved by transferring high nuclear spin polarization between inequivalent nuclei using cross polarization (CP) techniques. In addition, selective CP transfer can be applied as a useful tool for spectral editing. While $\mathrm{CP}$ is a very effective technique for static samples, the combination of $\mathrm{CP}$ with high-resolution solid-state NMR techniques that require sample rotation suffers from a number of difficulties. One of these difficulties is that the dipolar spin interactions that mediate the $\mathrm{CP}$ transfer become time dependent under magic-angle spinning (MAS) making the Hartmann-Hahn matching conditions more complicated and also reducing the 
efficiency of the polarization transfer. Another difficulty arises when cross polarizing the central transition of half-integer quadrupolar nuclei. In this situation the time-dependence of the large first-order quadrupolar interaction interferes with the Hartmann-Hahn matching. Remember that even though the central transition is unaffected to first-order by the quadrupolar interaction, the energy levels themselves are affected.

Dynamic Angle Spinning (DAS) NMR was designed to provide high resolution isotropic spectra for the central transition of half-integer quadrupolar nuclei that are broadened due to second-order effects. DAS achieves this line narrowing capability by making the angle of the spinner axis a time-dependent variable. This additional degree of freedom aids not only in providing high-resolution spectra, but, as we show here provides a solution to the problem of combining cross-polarization with high-resolution solid-state NMR techniques. This solution exploits the time independence of the spin eigenvalues when spinning at $0^{\circ}$ (parallel) to the external magnetic field direction. By performing the $\mathrm{CP}$ step while spinning at $0^{\circ}$, the full static $\mathrm{CP}$ intensity can he obtained and used in an MAS, variable-angle spinning (VAS), or DAS experiment.

\section{Theory}

The theory of spin locking and cross polarization of the central transition of halfodd integer nuclei has been described in detail by Vega ${ }^{96,105}$. In this section, we present a condensed treatment of this problem.

In the $\mathrm{CP}$ experiment involving polarization transfer from a spin $I=1 / 2$ to the central transition of a quadrupolar spin of $S=3 / 2$, the observable of interest, $\left\langle S_{+}(t)\right\rangle$, is obtained from the relation

$$
\left\langle S_{+}(t)\right\rangle=\operatorname{Tr}\left\{\sigma(t) S_{+}\right\}
$$

Here $\sigma(t)$ is the density operator whose evolution is given by 


$$
\sigma(t)=U(t) \sigma(0) U^{\dagger}(t)
$$

where

$$
U(t)=T \exp \left[-i \int_{0}^{t} H(s) d s\right],
$$

$T$ is the time ordering operator, and $H(t)$ is the Hamiltonian. The secular Hamiltonian in the rotating frame is given by

$$
H(t)=H_{R F}+H_{D}(t)+H_{Q}(t)
$$

where

$$
\begin{aligned}
& H_{R F}=-\hbar \omega_{1 /} I_{x}-\hbar \omega_{1 S} S_{x}, \\
& H_{D}(t)=\hbar \omega_{D} A_{20}^{D}(t) 2 I_{z} S_{z}
\end{aligned}
$$

and

$$
H_{Q}(t)=\hbar \omega_{Q} A_{20}^{Q}(t) \frac{1}{\sqrt{6}}\left(3 S_{z}^{2}-S(S+1)\right) \text {, }
$$

where $\omega_{1 /}$ and $\omega_{1 S}$ are the rf-field strengths for $I$ and $S$ spins, respectively, and $A_{20}^{D}(t)$ and $A_{20}^{Q}(t)$ are irreducible spherical tensors for the dipolar and quadrupolar interactions defined in chapter 2, respectively. It is convenient to rewrite this Hamiltonian in the fictitious spin-1/2 formalism ${ }^{26,35}$ (see chapter 2) as

$$
\begin{aligned}
H(t) & =-\hbar \omega_{1 I} I_{x}-\sqrt{3} \hbar \omega_{1 S}\left(S_{x}^{12}+S_{x}^{34}\right)-2 \hbar \omega_{1 S} S_{x}^{23} \\
& +\sqrt{6} \hbar \omega_{Q} A_{20}^{Q}(t)\left(S_{z}^{12}-S_{z}^{34}\right)+3 \hbar \omega_{D} A_{20}^{D}(t) 2 I_{z} S_{z}^{14} \\
& +\hbar \omega_{D} A_{20}^{D}(t) 2 I_{z} S_{z}^{23} .
\end{aligned}
$$

We assume $\left|\omega_{1 I}\right|,\left|\omega_{1 S}\right| \gg\left|\omega_{D}\right|$, and transform into a time dependent frame ${ }^{27}$ that diagonalizes $H_{R F}+H_{Q}(t)$ using

$$
\begin{aligned}
W(t) & =\exp \left(-i \frac{\pi}{2} I_{y}\right) \exp \left(i \frac{\pi}{2} S_{y}^{14}\right) \exp \left(-i \frac{\pi}{2} S_{y}^{23}\right) \\
& \times \exp \left(i 2 \xi_{1}(t) S_{y}^{13}\right) \exp \left(i 2 \xi_{2}(t) S_{y}^{24}\right)
\end{aligned}
$$


where

$$
\tan 2 \xi_{1}(t)=\frac{\sqrt{3} \omega_{1 S}}{-\sqrt{6} \omega_{Q^{2}} A_{20}^{Q}(t)+\omega_{1 S}}
$$

and

$$
\tan 2 \xi_{2}(t)=\frac{\sqrt{3} \omega_{1 S}}{-\sqrt{6} \omega_{Q} A_{20}^{Q}(t)-\omega_{1 S}}
$$

The propagator in this time dependent frame is given by

$$
\tilde{U}(t)=T \exp \left\{-\frac{i}{\hbar} \int_{0}^{t}\left[\tilde{H}(s)-i \hbar \dot{W}^{\dagger}(s) W(s)\right] d s\right\}
$$

where

$$
\begin{aligned}
\tilde{H}(t) & =W^{\dagger}(t) H(t) W(t) \\
& =-\hbar \omega_{1 I} I_{z}+\hbar \omega_{1 S}\left(S_{z}^{14}-S_{z}^{23}\right)-\hbar \omega_{13}(t) S_{z}^{13}-\hbar \omega_{24}(t) S_{z}^{24} \\
& -\hbar b_{I S}^{14}(t) 2 I_{x} S_{x}^{14}+\hbar b_{I S}^{23}(t) 2 I_{x} S_{x}^{23}-\hbar b_{I S}^{12}(t) 2 I_{x} S_{x}^{12}-\hbar b_{I S}^{34}(t) 2 I_{x} S_{x}^{34}
\end{aligned}
$$

with

$$
\begin{aligned}
& \omega_{13}(t)=\sqrt{3 \omega_{1 S}^{2}+\left(\sqrt{6} \omega_{Q} A_{20}^{Q}(t)-\omega_{1 S}\right)^{2}}=\frac{-\sqrt{6} \omega_{Q} A_{20}^{Q}(t)+\omega_{1 S}}{\cos 2 \xi_{1}(t)} \\
& \omega_{24}(t)=-\sqrt{3 \omega_{1 S}^{2}+\left(\sqrt{6} \omega_{Q} A_{20}^{Q}(t)+\omega_{1 S}\right)^{2}}=\frac{\sqrt{6} \omega_{Q} A_{20}^{Q}(t)+\omega_{1 S}}{\cos 2 \xi_{2}(t)} \\
& b_{I S}^{14}(t)=\omega_{D} A_{20}^{D}(t)\left\{2 \cos \left(\xi_{1}(t)-\xi_{2}(t)\right)+\cos \left(\xi_{1}(t)+\xi_{2}(t)\right)\right\} \\
& b_{I S}^{12}(t)=\omega_{D} A_{20}^{D}(t)\left\{2 \sin \left(\xi_{1}(t)-\xi_{2}(t)\right)-\sin \left(\xi_{1}(t)+\xi_{2}(t)\right)\right\} \\
& b_{I S}^{23}(t)=\omega_{D} A_{20}^{D}(t)\left\{2 \cos \left(\xi_{1}(t)-\xi_{2}(t)\right)-\cos \left(\xi_{1}(t)+\xi_{2}(t)\right)\right\} \\
& b_{I S}^{34}(t)=\omega_{D} A_{20}^{D}(t)\left\{2 \sin \left(\xi_{1}(t)-\xi_{2}(t)\right)+\sin \left(\xi_{1}(t)+\xi_{2}(t)\right)\right\}
\end{aligned}
$$

and

$$
i \hbar \dot{W}^{\dagger}(t) W(t)=2 \hbar \frac{d \xi_{1}(t)}{d t} S_{y}^{13}+2 \hbar \frac{d \xi_{2}(t)}{d t} S_{y}^{24}
$$


where $0 \leq\left\{\xi_{1}(t), \xi_{2}(t)\right\} \leq \frac{\pi}{2} . \quad \tilde{U}(t)$ is related to the rotating frame propagator by $U(t)=W(t) \tilde{U}(t) W^{\dagger}(0)$. Equation 5.1 can be rewritten

$$
\left\langle S_{+}(t)\right\rangle=\operatorname{Tr}\left\{\tilde{U}(t) \tilde{\sigma}(0) \tilde{U}^{\dagger}(t) \tilde{S}_{+}\right\},
$$

where $\tilde{S}_{+}=W^{\dagger}(t) S_{+} W(t)$ and $\tilde{\sigma}(0)=W^{\dagger}(0) \tilde{\sigma}(0) W(0)$. The transformed observable $\tilde{S}_{+}$ is

$$
\begin{aligned}
\tilde{S}_{+} & =S_{z}^{23} \cos ^{2} \xi_{1}(t) \cos ^{2} \xi_{2}(t)+S_{z}^{13} \sin 2 \xi_{1}(t) \\
& -S_{z}^{14} \sin ^{2} \xi_{1}(t) \sin ^{2} \xi_{2}(t)-S_{z}^{24} \sin 2 \xi_{2}(t) \\
& -S_{z}^{12} \sin ^{2} \xi_{1}(t) \cos ^{2} \xi_{2}(t)-S_{z}^{34} \cos ^{2} \xi_{1}(t) \sin ^{2} \xi_{2}(t) \\
& +S_{x}^{13}\left\{\cos \xi_{1}(t) \sin \xi_{1}(t)-\cos 2 \xi_{1}(t)\right\} \\
& +S_{x}^{24}\left\{\cos \xi_{2}(t) \sin \xi_{2}(t)+\cos 2 \xi_{2}(t)\right\} \\
& +i S_{y}^{12}\left\{\sin \xi_{1}(t) \cos \xi_{2}(t)+\cos \left(\xi_{1}(t)-\xi_{2}(t)\right)\right\} \\
& +i S_{y}^{34}\left\{\cos \left(\xi_{1}(t)-\xi_{2}(t)\right)-\cos \xi_{1}(t) \sin \xi_{2}(t)\right\} \\
& +i S_{y}^{14}\left\{\sin \xi_{1}(t) \sin \xi_{2}(t)-\sin \left(\xi_{1}(t)-\xi_{2}(t)\right)\right\} \\
& +i S_{y}^{23}\left\{\cos \xi_{1}(t) \cos \xi_{2}(t)-\sin \left(\xi_{1}(t)-\xi_{2}(t)\right)\right\}
\end{aligned}
$$

After an initial $\pi / 2$ pulse on the $I$ spin, the initial density operator is $\sigma(0)=I_{x}$, and the transformed initial density operator is

$$
\tilde{\sigma}(0)=W^{\dagger}(0) \sigma(0) W(0)=I_{z} .
$$

In the static case, a Hartmann-Hahn matching condition of $\omega_{1 /}=(S+1 / 2) \omega_{1 S}$ is employed and only those spins where $\left|\omega_{Q} A_{20}^{Q}(\alpha, \beta, \gamma, t)\right| \gg\left|\omega_{1 S}\right|$ (where the spatial dependence of $A_{20}^{Q}(t)$ is given explicitly in terms of the Euler angles of the principle axis frame) undergo polarization transfer to the central transition.

In the case of rotating samples under the above matching conditions, only those spins that satisfy $\left|\omega_{Q} A_{20}^{Q}(\alpha, \beta, \gamma, t)\right| \gg\left|\omega_{1 S}\right|$ or pass through this condition during a rotor period, will undergo $\mathrm{CP}$ transfer, as shown by Vega. For these spins the values of $\xi_{1}(t)$ and $\xi_{2}(t)$ have values close to either 0 or $\pi / 2$. When $\xi_{1}(t) \approx \xi_{2}(t) \approx 0, \omega_{13}(t)$ and 
$\omega_{24}(t)$ are approximated by $-\omega_{Q} A_{20}^{Q}(t)+\omega_{1 S}$ and $\omega_{Q} A_{20}^{Q}(t)+\omega_{1 S}$ respectively, and when $\xi_{1}(t) \approx \xi_{2}(t) \approx \frac{\pi}{2}$, are approximated by $\omega_{Q} A_{20}^{Q}(t)-\omega_{1 S}$ and $-\omega_{Q} A_{20}^{Q}(t)-\omega_{1 S}$ respectively. In this situation, the Hamiltonian in equation 5.11 can be rewritten so that the diagonal elements are in terms of single and triple quantum transitions:

$$
\begin{gathered}
\tilde{H}(\xi \approx 0)=-\hbar \omega_{1 I} I_{z}-2 \hbar \omega_{1 S} S_{z}^{23}+\sqrt{6} \hbar \omega_{Q} A_{20}^{Q}(t)\left(S_{00}^{14}-S_{00}^{23}\right)- \\
6 \hbar \omega_{D} A_{20}^{D}(t) I_{x} S_{x}^{14}+2 \hbar \omega_{D} A_{20}^{D}(t) I_{x} S_{x}^{23}
\end{gathered}
$$

or

$$
\begin{gathered}
\tilde{H}\left(\xi \approx \frac{\pi}{2}\right)=-\hbar \omega_{1 I} I_{z}+2 \hbar \omega_{1 S} S_{z}^{14}-\sqrt{6} \hbar \omega_{Q} A_{20}^{Q}(t)\left(S_{00}^{14}-S_{00}^{23}\right)- \\
2 \hbar \omega_{D} A_{20}^{D}(t) I_{x} S_{x}^{14}+6 \hbar \omega_{D} A_{20}^{D}(i)(t) I_{x} S_{x}^{23}
\end{gathered}
$$

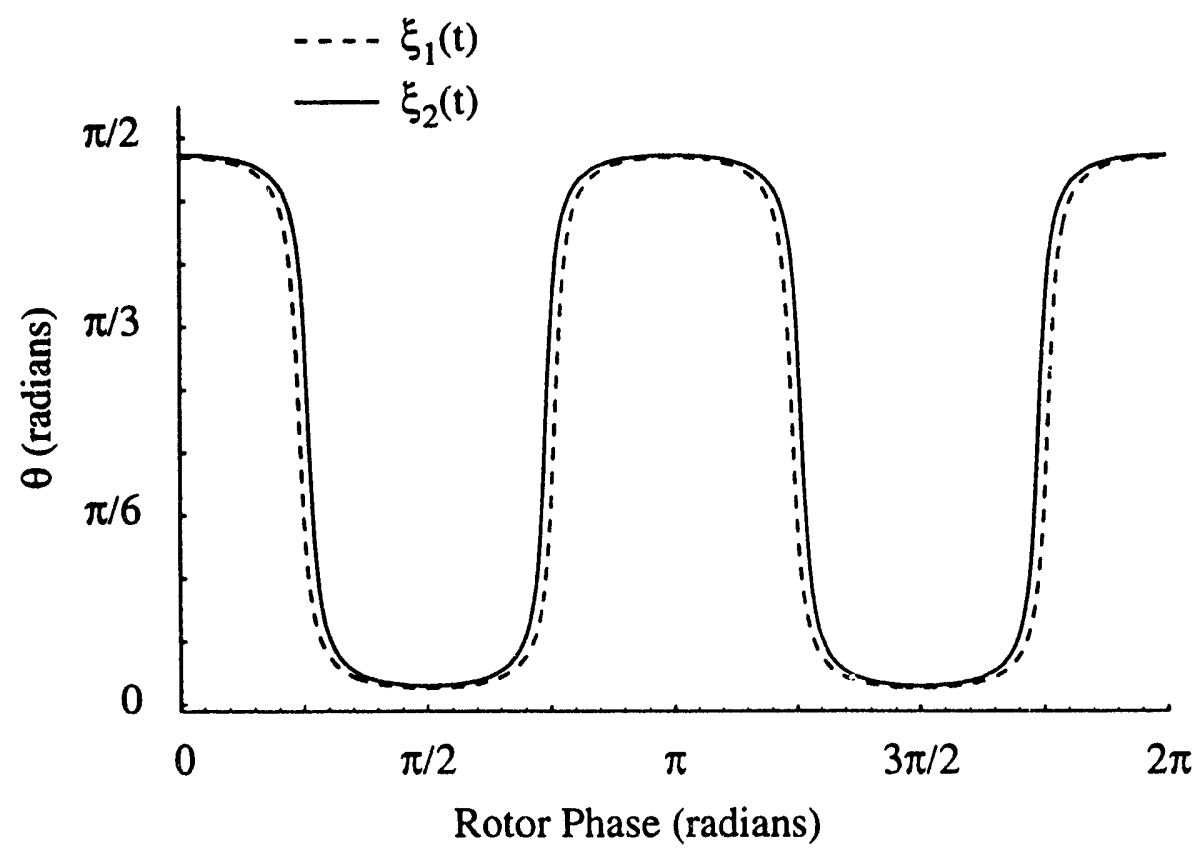

Figure 5.1 $\xi_{1}$ and $\xi_{2}$ as a Function of Rotor Phase. The values of both $\xi_{1}$ and $\xi_{2}$ stay very near their minimum $(0)$ and maximum $(\pi / 2)$ values for all rotor phases, indicating that the approximations made for equation 5.24 and 5.25 are quite reasonable.

Figure 5.1 shows the graph of $\xi_{1}$ and $\xi_{2}$ as a function of rotor phase for a crystallite with a quadrupolar coupling constant of $11.0 \mathrm{MHz}$, asymmetry parameter of 0.0 whose PAS is oriented perpendicular to the rotor which is spinning about an axis oriented $54.74^{\circ}$ from 
the magic angle. Notice that the values of stay very close to the minimum and maximum values at almost all rotor phases. These curves are typical for most crystallite orientations. The Hamiltonians in equation 5.24 and 5.25 may be transformed into the RF rotating frame, assuming that $\left|\omega_{1 I}\right|,\left|\omega_{1 S}\right| \gg\left|\omega_{D}\right|$, as has been previously discussed.92,94

$$
\begin{aligned}
& I_{x} \rightarrow I_{x} \cos \omega_{11} t+I_{y} \sin \omega_{1 I} t \\
& S_{x}^{23} \rightarrow S_{x}^{23} \cos 2 \omega_{1 S} t+S_{y}^{23} \sin 2 \omega_{1 S} t \\
& \tilde{H}(\xi \approx 0)= \hbar \omega_{Q} A_{20}^{Q}(t)\left(S_{00}^{14}-S_{00}^{23}\right) \\
&-6 \hbar \omega_{D} A_{20}^{D}(t)\left(I_{x} \cos \omega_{1 I} t+I_{y} \sin \omega_{1 I} t\right) S_{x}^{14} \\
&+2 \hbar \omega_{D} A_{20}^{D}(t)\left(I_{x} \cos \omega_{1 I} t+I_{y} \sin \omega_{1 I} t\right) \\
& \times\left(S_{x}^{23} \cos 2 \omega_{1 S} t+S_{y}^{23} \sin 2 \omega_{1 S} t\right)
\end{aligned}
$$

or

$$
\begin{aligned}
& I_{x} \rightarrow I_{x} \cos \omega_{1 /} t+I_{y} \sin \omega_{1 /} t \\
& S_{x}^{14} \rightarrow S_{x}^{14} \cos 2 \omega_{1 S^{t}}-S_{y}^{14} \sin 2 \omega_{1 S} t \\
& \tilde{H}\left(\xi \approx \frac{\pi}{2}\right)=-\hbar \omega_{Q} A_{20}^{Q}(t)\left(S_{00}^{14}-S_{00}^{23}\right) \\
&+6 \hbar \omega_{D} A_{20}^{D}(t)\left(I_{x} \cos \omega_{1 /} t+I_{y} \sin \omega_{1 I} t\right) S_{x}^{23} \\
&-2 \hbar \omega_{D} A_{20}^{D}(t)\left(I_{x} \cos \omega_{1 /} t+I_{y} \sin \omega_{1 I} t\right) \\
& \times\left(S_{x}^{14} \cos 2 \omega_{1 S} t-S_{y}^{14} \sin 2 \omega_{1 S} t\right)
\end{aligned}
$$

Under the Hartmann-Hahn condition for the central transition, $\omega_{1 I}=2 \omega_{1 S}$, these equations simplify into terms which oscillate at frequencies of $0, \omega_{1 /}$ or $2 \omega_{1 l}$. The time dependent cosine and sine modulated terms that remain will vanish in the time average between zero crossings when $\omega_{1 I}>\omega_{r}$ simplifying the Hamiltonians in the doubly rotating frame further.

$$
\tilde{H}(\xi \approx 0)=\sqrt{6} \hbar \omega_{Q} A_{20}^{Q}(t)\left(S_{00}^{14}-S_{00}^{23}\right)+\hbar \omega_{D} A_{20}^{D}(t)\left(I_{x} S_{x}^{23}+I_{y} S_{y}^{23}\right)
$$

or 


$$
\tilde{H}\left(\xi \approx \frac{\pi}{2}\right)=-\sqrt{6} \hbar \omega_{Q} A_{20}^{Q}(t)\left(S_{00}^{14}-S_{00}^{23}\right)-\hbar \omega_{D} A_{20}^{D}(t)\left(I_{x} S_{x}^{14}-I_{y} S_{y}^{14}\right)
$$

Additionally, the transformed observable, $\tilde{S}_{+}$, of Eq. [23] becomes

$$
\begin{aligned}
\tilde{S}_{+} & =S_{z}^{23} \cos ^{2} \xi_{1}(t) \cos ^{2} \xi_{2}(t)-S_{z}^{14} \sin ^{2} \xi_{1}(t) \sin ^{2} \xi_{2}(t) \\
& +S_{x}^{24} \cos 2 \xi_{2}(t)-S_{x}^{13} \cos 2 \xi_{1}(t) \\
& +i S_{y}^{23} \cos \xi_{1}(t) \cos \xi_{2}(t)+i S_{y}^{14} \sin \xi_{1}(t) \sin \xi_{2}(t) \\
& +i S_{y}^{12} \cos \left(\xi_{1}(t)-\xi_{2}(t)\right)+i S_{y}^{34} \cos \left(\xi_{1}(t)-\xi_{2}(t)\right)
\end{aligned}
$$

In general, the time-ordering operator $T$ makes the derivation of $\tilde{U}(t)$ in Eq. [20] complicated since $\tilde{H}(t)$ and $i \hbar \dot{W}^{\dagger}(t) W(t)$ do not commute with themselves or each other at all times. There are however certain approximations that can simplify this task. How the system evolves depends on whether the passage through or near the zero crossing is adiabatic or sudden. In figure 5.2 the values of $\omega_{13}(t), \omega_{24}(t), 2 d \xi_{1} / d t$ and $2 d \xi_{2} / d t$ are shown plotted versus $\sqrt{6} \omega_{Q} A_{20}^{Q}(t)$ for three different spinning rates $(5 \mathrm{kHz}, 1 \mathrm{kHz}$ and $100 \mathrm{~Hz}$ ). It can be seen that the off diagonal $i \hbar \dot{W}^{\dagger}(t) W(t)$ terms are only important when the spinning is rapid and the size of the quadrupolar coupling is small. The simulation parameters are identical to those used in figure 5.1.

The adiabatic approximation is permitted when $|\tilde{H}(t)| \gg\left|\dot{W}^{\dagger}(t) W(t)\right|$ at all times (as in figure $5.2,100 \mathrm{~Hz}$ spinning rate), and the propagator becomes

$$
\tilde{U}_{a}(t)=\exp \left[-\frac{i}{\hbar} \int_{0}^{t} \tilde{H}(s) d s\right] .
$$

Under this propagator, with the Hartmann-Hahn match given above, the time dependent rotating frame initial density matrix (equation 5.24) becomes

$$
\tilde{U}_{a}(t) \tilde{\sigma}(0) \tilde{U}_{a}^{\dagger}(t)=\tilde{U}_{a}(t) I_{z} \tilde{U}_{a}^{\dagger}(t) \rightarrow \frac{1}{2}\left(I_{z}+S_{z}^{23}\right)
$$

(assuming we start with a crystallite with $\xi_{1}(t) \approx \xi_{2}(t) \approx 0$ ) and polarization is transferred from the $I$-spin to the central transition of the $S$-spin. When the sample rotation takes the first-order quadrupolar coupling through or near zero, CP transfer from the $I$ - 
spin to the central transition of the $S$-spin continues, since the system is under adiabatic conditions.
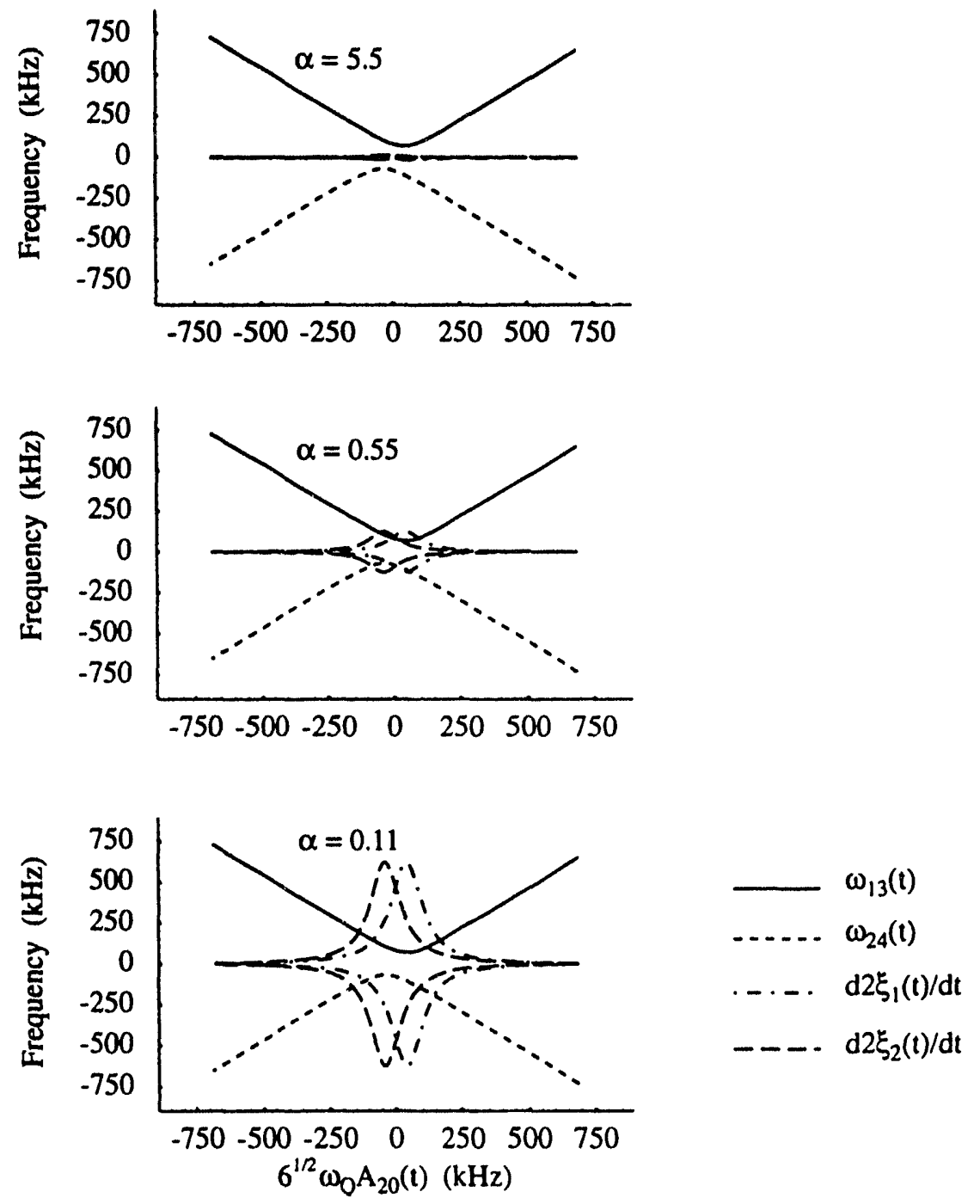

Figure 5.2 Matrix elements in cross-polarization of quadrupolar nuclei. The spinning rates are $5 \mathrm{kHz}, 1 \mathrm{kHz}$ and $100 \mathrm{~Hz}$ which produce adiabaticity parameters of $0.11,0.55$ and 5.5 respectively. Notice that the off-diagonal terms dominate only in the small quadrupolar coupling region and in cases of rapid spinning

Before the zero-crossing, $\xi_{1}(t) \approx \xi_{2}(t) \approx 0$ and the effective observable is therefore $\tilde{S}_{+}^{23}=S_{z}^{23}+i S_{y}^{23}$ (as can be seen from equation 5.23) resulting in a large observable signal. After the zero-crossing, $\xi_{1}(t) \approx \xi_{2}(t) \approx \frac{\pi}{2}$ and the effective observable is $\tilde{S}_{+}^{23}=-S_{z}^{14}+i S_{y}^{14}$. Therefore, the cross-polarized central-transition observable intensity $\left\langle S_{+}^{23}(t)\right\rangle$ goes to zero. However, since $\xi_{1}(t) \approx \xi_{2}(t) \approx \frac{\pi}{2}$, the effective adiabatic 
Hamiltonian is changed to Eq. [30] and the polarization will transfer from the $I$-spin to the triple quantum $S$-spin coherence in the transformed frame. Any central coherence polarization will remain spin locked and unchanged.

$$
\tilde{U}_{a}(t) I_{z} \tilde{U}_{a}^{\dagger}(t) \rightarrow \frac{1}{2}\left(I_{z}+S_{z}^{14}\right)
$$

This evolved coherence is exactly of the same form as our observable operator, and leads to observable intensity identical to immediately prior to the first zero crossing. After the next zero-crossing, the effective observable is transformed back into $\tilde{S}_{+}^{23}=S_{z}^{23}+i S_{y}^{23}$, and the central-transition begins to cross polarize again while the triple quantum coherence remains spin locked. After multiple zero-crossing cycles in the thermodynamic limit, the central and triple quantum transitions will be equally polarized from the $I$-spin as shown by Vega ${ }^{105}$. The state of the observable does not matter for observable intensity at this point. The overall $\mathrm{CP}$ intensity will be identical to that observed for a static spin in the thermodynamic limit, however the overall rate will be half as fast, since both the central and triple quantum transitions are being polarized simultaneously. In the presence of a short rotating frame relaxation time, this will lead to a reduced overall $\mathrm{CP}$ intensity from adiabatic spins.

The sudden approximation is permitted when (as in figure $5.2,5 \mathrm{kHz}$ spinning speed) at the zero-crossing, $|\tilde{H}(t)| \ll\left|\dot{W}^{\dagger}(t) W(t)\right|$. The propagator is then

$$
\tilde{U}_{s}(t)=\exp \left[\int_{0}^{t} \dot{W}^{\dagger}(s) W(s) d s\right] .
$$

This propagator transforms the $S_{z}^{23}$ and $S_{z}^{14}$ in the following manner:

$$
\begin{aligned}
& \tilde{U}_{s}(t) S_{z}^{23} \tilde{U}_{s}^{\dagger}(t) \rightarrow S_{z}^{14} \\
& \tilde{U}_{s}(t) S_{z}^{14} \tilde{U}_{s}^{\dagger}(t) \rightarrow S_{z}^{23}
\end{aligned}
$$

The transformed initial density operator before the first zero-crossing is still given by equation 5.32. However, during the first zero-crossing, the $S_{z}^{23}$ term is transformed to $S_{z}^{14}$. 
In the sudden reversal of the first-order quadrupolar coupling, the cross-polarized centraltransition is transferred to the triple quantum transition after the reversal. The observable intensity after the zero-crossing is therefore identical to that immediately before the zerocrossing. After the first zero-crossing, the $\mathrm{CP}$ transfer from the I-spins to the triple quantum coherence will continue according to equation 5.33. Therefore the observable operator will always match the cross polarizing transition. After multiple zero crossings, one of the two transitions will be completely polarized while the other/ will be unpolarized. The polarized intensity will always remain observable and the CP efficiency and rate should be identical to the static case.

For crystallites which pass through the zero crossing in neither an adiabatic or sudden regime fall into the intermediate regime (see figure $5.2,1 \mathrm{kHz}$ spinning speed). This type of evolution is the most difficult of the three cases to calculate. To determine the evolution of the density matrix in the intermediate regime, we need to include both the diagonal $\tilde{H}(t)$ and the off diagonal $\dot{W}^{\dagger}(t) W(t)$ contributions to the unitary evolution propagator. These two terms do not commute with each other and the time ordering operator may not be easily removed. The solution to this problem is to rediagonalize the net Hamiltonian at each time step. There does not appear to be an easy method for doing this diagonalization. Vega has shown with numerical simulations that spins undergoing an intermediate regime zero crossing evolve into non-spin locked states ${ }^{96,105}$. Therefore, the contribution these spins make will only be prior to their first zero-crossing, after which their contribution to the overall observed cross polarization intensity will decay rapidly.

We have performed variable spinning angle cross-polarization experiments which may be approximately described with the above results. For a powdered sample, we may classify each spin according to its PAS orientation with respect to the rotor in one of five categories. These categories are: 
1. Spins with large $\omega_{Q} A_{20}^{Q}(t)$ which undergo no zero crossings (essentially static spins, alternatively spins which have $\xi_{1}$ and $\xi_{2}$ approximately equal to either 0 or $\pi / 2$ for all time),

2. Spins which have a small $\omega_{Q} A_{20}^{Q}(t)$ for the majority of a rotor period (alternatively those spins which only have small oscillations in $\xi_{1}$ and $\xi_{2}$ about $\pi / 4$ ),

3. Spins which have a large $\omega_{Q} A_{20}^{Q}(t)$ for most of the rotor period and undergo adiabatic regime zero crossings (alternatively those spins for which $\xi_{1}$ and $\xi_{2}$ oscillate between 0 and $\pi / 2$ ),

4. Spins which have a large $\omega_{Q} A_{20}^{Q}(t)$ for most of the rotor period (just as in 3) and undergo sudden regime zero crossings,

5. Spins which have a large $\omega_{Q} A_{20}^{Q}(t)$ for most of the rotor period (just as in 3 and 4) and undergo intermediate regime zero crossings.

For spins which fall into the first and fourth categories, the cross polarization contributions are simple, as they will contribute full intensity with normal polarization build up rates. For the spins in the fifth category, the cross polarization contribution is also simple to calculate, since in the limit of long contact times, they will contribute no cross polarization intensity. For spins in the second category, the Hartmann-Hahn match condition will not be met for a significant portion of the total contact time and the contribution will again to zero to the overall cross polarization intensity. For spins in the third category, the cross polarization contribution will be identical to the static or sudden spins, however the build up rate will be half as fast.

The only difficulty remaining is to determine mathematically the definition for each of these five categories. The first category is the most easy to define, as this constitutes spins for which $\left|\sqrt{6} \omega_{Q} A_{20}^{Q}(t)\right|>5 \omega_{15}$ at all times (corresponding to $\xi_{1}$ and $\xi_{2}$ within $\pi / 12$ of the minima and maxima of 0 or $\pi / 2$ ). The extent to which the quadrupolar coupling must be larger than the radio frequency strength is difficult to define exactly, but 
generally a factor of five or more is probably sufficient to fully truncate the radio frequency portion of the Hamiltonian. The second category is likewise simple in definition, as this represents the spins for which $\left|\sqrt{6} \omega_{Q} A_{20}^{Q}(t)\right| \leq 5 \omega_{1 S}$ for more than one third of a rotor period. The factor of one third is an arbitrary number which seems to work well in practice. This in general will represent only the spins whose PAS z-axis lies near the magic-angle under sample rotation. The third, fourth and fifth categories relate to spins which spend a majority of their time (more than two-thirds of a rotor period) with $\left|\sqrt{6} \omega_{Q} A_{20}^{Q}(t)\right|>5 \omega_{1 S}$. To differentiate between these three cases, the ratio of the sizes of $|\tilde{H}(t)|$ and $\left|\dot{W}^{\dagger}(t) W(t)\right|$ at the zero crossing must be considered. This leads to the definition of an adiabaticity parameter $\alpha$ below.

$$
\begin{aligned}
& |\tilde{H}(t)| \approx \sqrt{3 \omega_{1 S}^{2}+\left(\sqrt{6} \omega_{Q} A_{20}^{Q}(t)-\omega_{1 S}\right)^{2}} \\
& \left|\dot{W}^{\dagger}(t) W(t)\right| \approx 2 \frac{d \xi_{1}(t)}{d t}=\frac{\sqrt{18} \omega_{1 S} \omega_{Q}}{3 \omega_{1 S}^{2}+\left(\sqrt{6} \omega_{Q} A_{20}^{Q}(t)-\omega_{1 S}\right)^{2}} \frac{d A_{20}^{Q}(t)}{d t} \\
& \alpha=\frac{\left(3 \omega_{1 S}^{2}+\left(\sqrt{6} \omega_{Q} A_{20}^{Q}\left(t_{0}\right)-\omega_{1 S}\right)^{2}\right)^{3 / 2}}{\left.\sqrt{18} \omega_{1 S} \omega_{Q} \frac{d A_{20}^{Q}(t)}{d t}\right|_{t_{0}}}
\end{aligned}
$$

This is then evaluated at the zero crossing where $\sqrt{6} \omega_{Q} A_{20}^{Q}\left(t_{0}\right) \approx \omega_{1 S}$. At this point the value of $\xi_{1}$ goes through $\pi / 4$ and the adiabaticity parameter is then

$$
\alpha=\frac{3 \omega_{1 S}^{2}}{\left.\sqrt{6} \omega_{Q} \frac{d A_{20}^{Q}(t)}{d t}\right|_{t_{0}}}
$$

Alternatively one could define an adiabaticity factor based on $\omega_{24}$ and $2 d \xi_{2} / d t$, however this gives an identical result at the $2 d \xi_{2} / d t$ resonance where $\sqrt{6} \omega_{Q} A_{20}^{Q}\left(t_{0}\right) \approx-\omega_{1 S}$. Explicitly evaluating this derivative yields 


$$
\begin{aligned}
A_{20}^{Q}(t) & =\sum_{m=-2}^{2} \sum_{n=-2}^{2} e^{-i\left(m \omega_{r} t+n \alpha+m \gamma\right)} d_{m 0}^{(2)}(\theta) d_{n m}^{(2)}(\beta) \rho_{2 n} \\
\frac{d A_{20}^{Q}(t)}{d t} & =\omega_{r} \sum_{m=-2}^{2} \sum_{n=-2}^{2}-i m e^{-i\left(m \omega_{r} t+n \alpha+m \gamma\right)} d_{m 0}^{(2)}(\theta) d_{n m}^{(2)}(\beta) \rho_{2 n} \\
& =\omega_{r} B_{20}^{Q}(t) \\
\alpha & =\frac{3 \omega_{1 S}^{2}}{\omega_{Q} \omega_{r} B_{20}^{Q}\left(t_{0}\right)}
\end{aligned}
$$

This definition of adiabaticity parameter is proportional to the one used by Vega in his description of cross polarization ${ }^{105}$, however, now there is an additional orientation dependence as well which comes from the time derivative of $A_{20}^{Q}(t)$. When the value of $\alpha$ is much larger than one, then the diagonal terms dominate in the evolution and the spin will be categorized in group three (adiabatic). When the value of $\alpha$ is much less than one, then the off-diagonal terms dominate at the zero crossing and the spin will be categorized in group four (sudden). When $\alpha$ is of the order of one, then the spins are classified as group five (intermediate). To calculate the approximate cross polarization efficiency at a given spinning angle, we merely calculate the number of spins in each category and add the cross polarization intensity proportionally for each category. The adiabatic contribution is the most difficult to estimate as the build up rate is half as fast and therefore may not be fully cross polarized before rotating frame relaxation begins to impede the buildup. In any case the adiabatic contribution should lie somewhere between the $50 \%$ and $100 \%$ intensity contribution levels.

The theory for the dynamic-angle spinning experiment has been described previously in chapter 3 . Remember that in the DAS experiment, there exists a continuous distribution of angle pairs which lead to high resolution isotropic spectra. Specifically, the $k$ $=1$ and $k=5$ angle pairs will be evaluated under CP conditions. I have done experiments which compare $\mathrm{CP}$ efficiency at a variety of spinning angles. 


\section{Experiments on Sodium Pyruvate and Sodium Hydroxide}

The cross polarization experiments were performed on a home built spectrometer based on a Techmag acquisition system at $7.04 \mathrm{~T}\left({ }^{1} \mathrm{H}\right.$ NMR frequency of $301.200 \mathrm{MHz}$ and ${ }^{23} \mathrm{Na}$ frequency of $79.671 \mathrm{MHz}$ ).

(a)

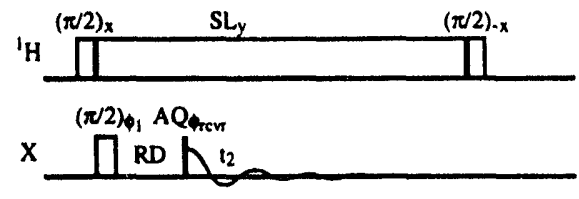

(b)

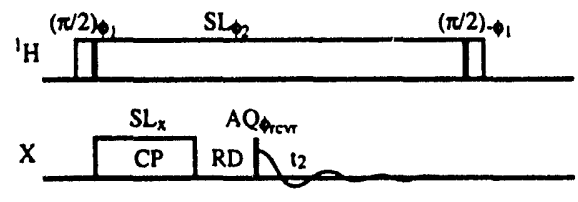

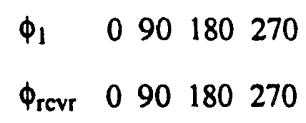

$\phi_{1} \quad 0 \quad 180$

$\phi_{2} \quad 90270$

$\phi_{\text {revr }} \quad 0 \quad 180$

(c)

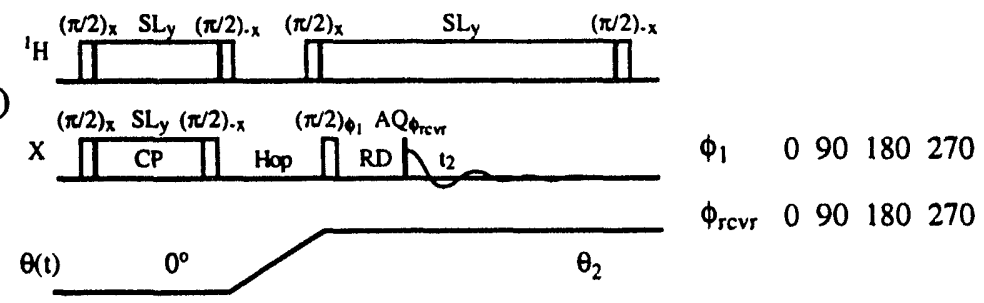

(d)
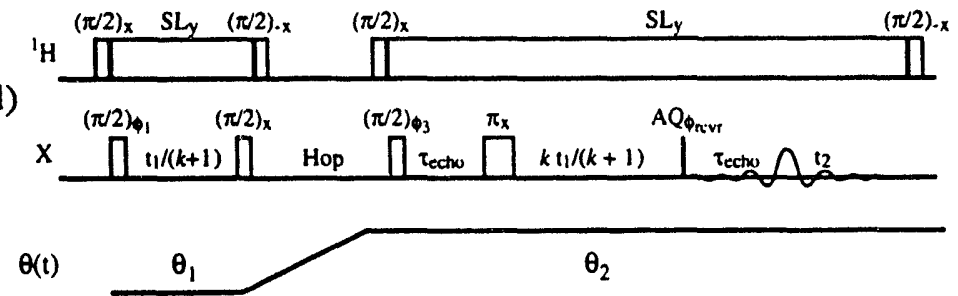

(e)

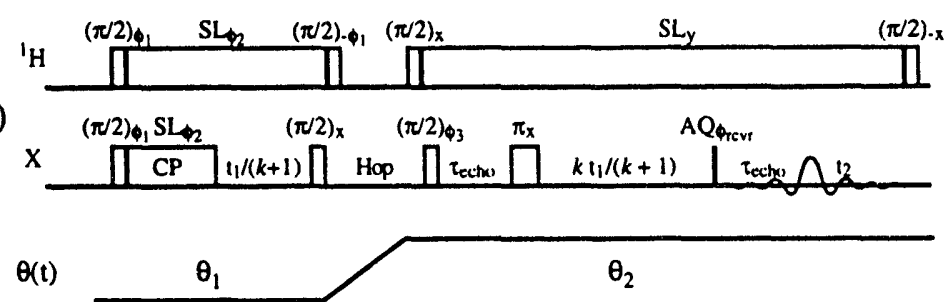

Figure 5.3 Cross-Polarization Pulse Sequences. Phase cycles are indicated for the one dimensional experiments (a-c) which are one pulse with decoupling, cross polarized with decoupling and zero-polarized with decoupling and sample spinning axis reorientation. The (d-e) pulse sequences are the normal and CP two dimensional DAS experiments respectively. The phase of the $90^{\circ}$ pulses on $\mathrm{X}$ are identical to those in the SEDAS experiment. The spin lock pulse is always set to the phase of the first pulse plus $90^{\circ}$. 
The DAS probe was home built with a stationary 0.75 " diameter coil for both rf-transmission and detection as described by Muellet et al. ${ }^{51}$ The double-tuned resonant circuit was similar to one described by Doty et al. ${ }^{107}$ The spinning rate was between 4.0 and 6.6 $\mathrm{kHz}$. The samples of sodium hydroxide, $\mathrm{NaOH} \cdot \mathrm{xH}_{2} \mathrm{O}$, and sodium pyruvate, $\mathrm{CH}_{3} \mathrm{OCOONa}$, used for these experiments were obtained from standard commercial sources. The pulse programs and phase cycles are given in figure 5.3 below. The DAS pulse sequence has been described previously in chapter 4 . For the $\mathrm{CP}$ efficiency' experiments, phase alternation of the ${ }^{1} \mathrm{H}_{\mathrm{ii}}$ was used (figure $5.3 \mathrm{~b}$ ) to assure that only the intensity due to $\mathrm{CP}$ would be measured. For CPDAS and ZPVAS experiments (figure 5.3a and 5.3c), a $90^{\circ}$ pulse was applied on ${ }^{23} \mathrm{Na}$ simultaneously with the initial ${ }^{1} \mathrm{H} 90^{\circ}$ to achieve the largest final sodium polarization. For the ${ }^{23} \mathrm{Na}$ spectra without $\mathrm{CP}$, recycle delays of $30 \mathrm{~s}$ and $16 \mathrm{~s}$ were used for $\mathrm{NaOH}$ and $\mathrm{CH}_{3} \mathrm{OCOONa}$, respectively, while, for the $\mathrm{CP}$ experiments, recycle delays of 10 minutes and $36 \mathrm{~s}$ were used, respectively. For the DAS experiments, we acquired 32 scans plus 1 dummy scan for each of the $90 t_{1}$ points while for the CP build up curves and ZPVAS spectra we acquired either 4, 8, or 64 scans plus 2 dummy scans for each different contact time and angle pair respectively. For the CPDAS and ZPVAS experiments on $\mathrm{CH}_{3} \mathrm{OCOONa}$ the $\mathrm{CP}$ contact time was $20 \mathrm{~ms}$. The contact time for $\mathrm{NaOH} 2 \mathrm{~ms}$. The input power of $200 \mathrm{~W}$ on the ${ }^{1} \mathrm{H}$ channel and $100 \mathrm{~W}$ on the ${ }^{23} \mathrm{Na}$ channel gave $7 \mu$ s central transition selective $90^{\circ}$ pulses. The $\mathrm{CP}$ Hartmann-Hahn match condition was achieved by setting $\gamma_{H} B_{1, H}=(I+1 / 2) \gamma_{N a} B_{1,2}=2 \gamma_{N a} B_{1, N a}$ which will selectively polarize the central $(1 / 2 \leftrightarrow-1 / 2)$ transition since the central transition nutation frequency is $\omega_{\text {nutation }}=(S+1 / 2) \gamma_{N a} B_{1, N a}$ in the presence of a large quadrupolar interaction $6,27,28,35$. Methods of simulating powder patterns have been described previously in chapter 2 and in additional papers ${ }^{41,76,81}$. 


\section{Cross Polarization Results and Discussion}

The effect of level crossings on CP efficiency can be seen clearly below in figure 5.4, which shows the cross polarization efficiencies of $\mathrm{NaOH}$ and sodium pyruvate versus VAS spinning angle. All intensities are scaled relative to the corresponding single pulse ${ }^{23} \mathrm{Na}$ VAS and MAS spectra, using the sequence in figure 5.3a.
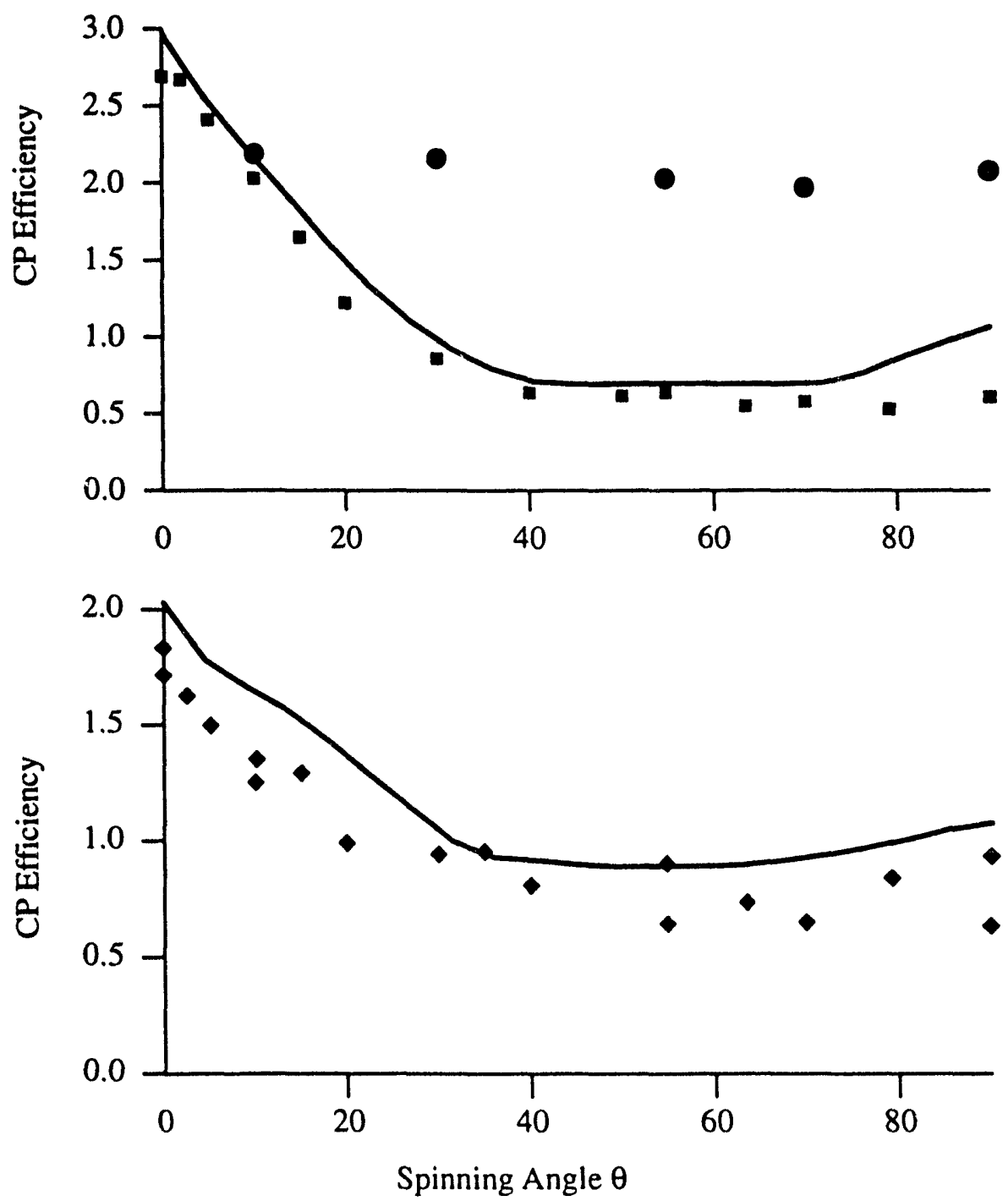

Figure 5.4 CP Efficiency versus Spinning Angle. The upper graph shows the cross polarization efficiency (boxes) for sodium pyruvate. The circles in this graph indicate the effective polarization under ZPVAS (rather than CPVAS). The line indicates the theoretical curve from the model described in the previous section. The lower graph shows the $\mathrm{CP}$ efficiency (diamonds) for sodium hydroxide. The line again represents the approximate theoretical efficiency. 
As expected, only under static $\left(0^{\circ} \mathrm{VAS}\right)$ conditions do we achieve the expected $\mathrm{CP}$ efficiency maximum of approximately $\gamma_{1} / 2 \gamma_{2} \approx 1.9$ for sodium hydroxide $(\mathrm{NaOH})$ and $3 \gamma_{1} / 4 \gamma_{2} \approx 2.84$ for sodium pyruvate $\left(\mathrm{CH}_{3} \mathrm{OCOO}^{-} \mathrm{Na}^{+}\right)$. The factor of $1 / 2$ and $3 / 4$ are due to the high abundance (basically $100 \%$ ) of both the ${ }^{1} \mathrm{H}$ and ${ }^{23} \mathrm{Na}$ isotopes causing cross polarization to be controlled by the equilibrium between their respective spin temperatures and heat capacities (related to the number of protons and sodium atoms per molecule). As the VAS angle increases, CP efficiency decreases dramatically. As seen in figure 5.4, spinning the sample at an angle greater than approximately $30^{\circ}$ results in a $\mathrm{CP}$ efficiency that is less than that achieved by a single pulse. This indicates that the level crossings are significant, even when only a reduced fraction of the spins are undergoing the maximum four crossings per rotor cycle. For DAS purposes, the only angle pairs which will have an angle less than $30^{\circ}$ will be those with high $k$ values. This immediately points to the $k=5$ experiment, since this has added advantages of fastest effective spinning rate and narrowest homonuclear dipolar linewidths (see chapter 3).

The dashed theoretical fits in figure 5.4 were obtained by numerically calculating the curves according to the theory outlined in the previous section. For $\mathrm{NaOH}$ and $\mathrm{CH}_{3} \mathrm{OCOONa}$, the values of $e^{2} q Q / h$ were 1.8 and $2.36 \mathrm{MHz}$ and $\eta_{\mathrm{Q}}$ were 0.0 and 0.77 respectively. The parameters for sodium pyruvate were obtained from simulations of the MAS spectrum while those of sodium hydroxide were taken from Vega. ${ }^{105}$ Qualitatively, the theoretical CP efficiency curves are approximately what one would expect, with the greatest CP enhancement for VAS angles near $0^{\circ}$.

Figure 5.4 also shows CP efficiency for sodium pyruvate at the angle at which detection occurred under ZPVAS. Since CP always occurs at $0^{\circ}$, the observed efficiency is constant for all angles. However, the efficiency under ZPVAS is less than that observed under $0^{\circ} \mathrm{CPVAS}$ because of $T_{1}$ relaxation processes that occur during the hop from $0^{\circ}$ to the detection angle. 
In figure 5.5 we show the ${ }^{1} \mathrm{H}$ decoupled MAS spectra of sodium pyruvate acquired with and without CP and with ZPMAS along with the simulation of the MAS powder pattern. The signal-to-noise is the worst for CPMAS-about $75 \%$ of that seen in the MAS spectrum without $\mathrm{CP}$.

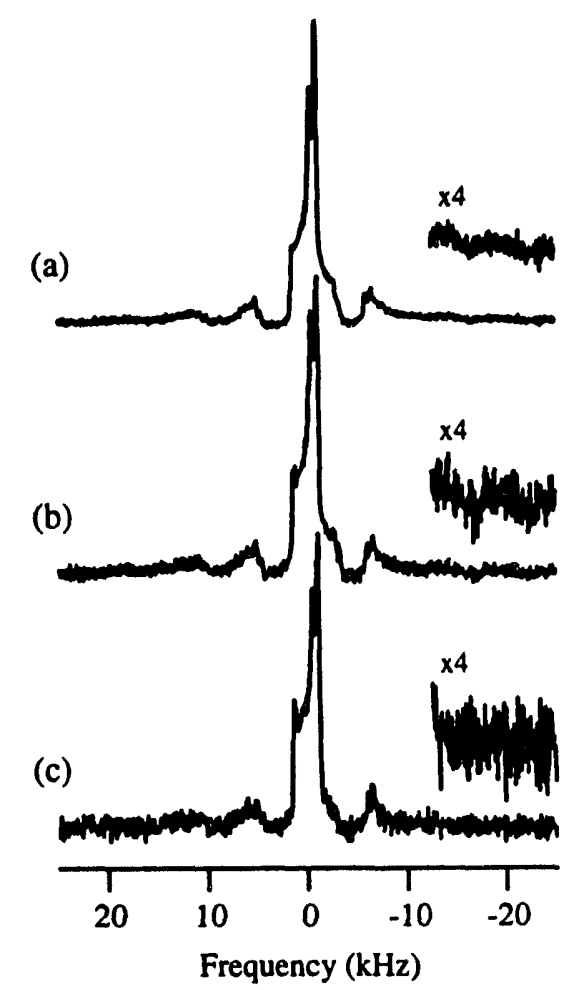

(d)

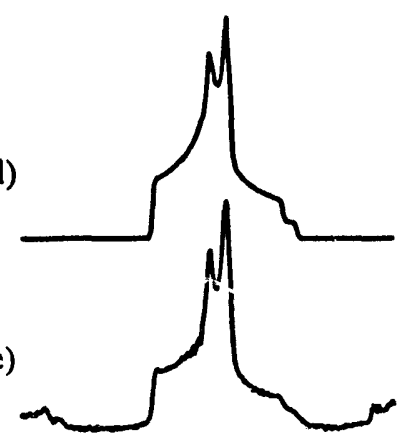

(f)
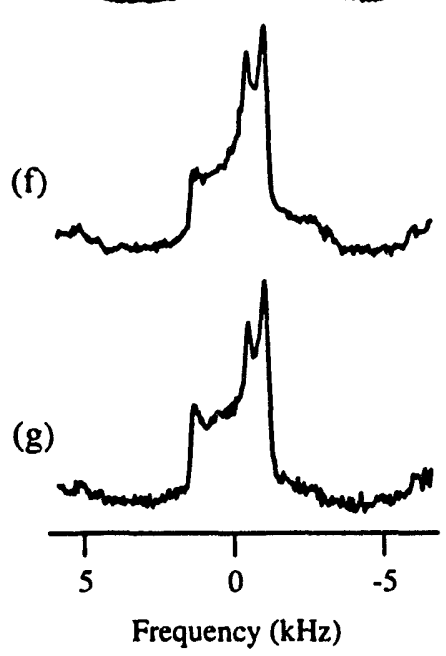

Figure 5.5 Comparison of CPMAS, MAS and ZPMAS Experiments. (a) and (e) show ZPMAS spectra, (b) and (f) show 1-pulse MAS spectra, (c) and (g) show CPMAS spectra and (d) shows the high speed MAS simulation.

On the other hand, the ZPMAS spectrum has a signal-to-noise ratio about twice that seen in the MAS spectrum taken without CP. This is expected since very little of the static cross polarized magnetization should decay by $\mathrm{T}_{1}$ processes during the hop from $0^{\circ}$ to $54.74^{\circ}$ while the cross polarization efficiency under MAS is so poor.

In figure 5.6, the decoupled DAS and CPDAS spectra of sodium pyruvate for the $0^{\circ}-63.43^{\circ}(k=5)$ and $37.38^{\circ}-79.19^{\circ}(k=1)$ angle pairs are compared. As can be seen in figure 5.4, for $k=5$, we observe over 2.5 times the signal-to-noise in the spectrum taken 
with CP compared to the spectrum taken without $\mathrm{CP}$. In addition, the CPDAS experiment at $k=5$ has a S/N ratio over 4.5 times that of the CPDAS experiment at $k=1$. This demonstrates the importance of $0^{\circ}$ cross polarization for DAS. The CPDAS experiment done at $37.38^{\circ}(k=1)$ has a worse $\mathrm{S} / \mathrm{N}$ than the same experiment done

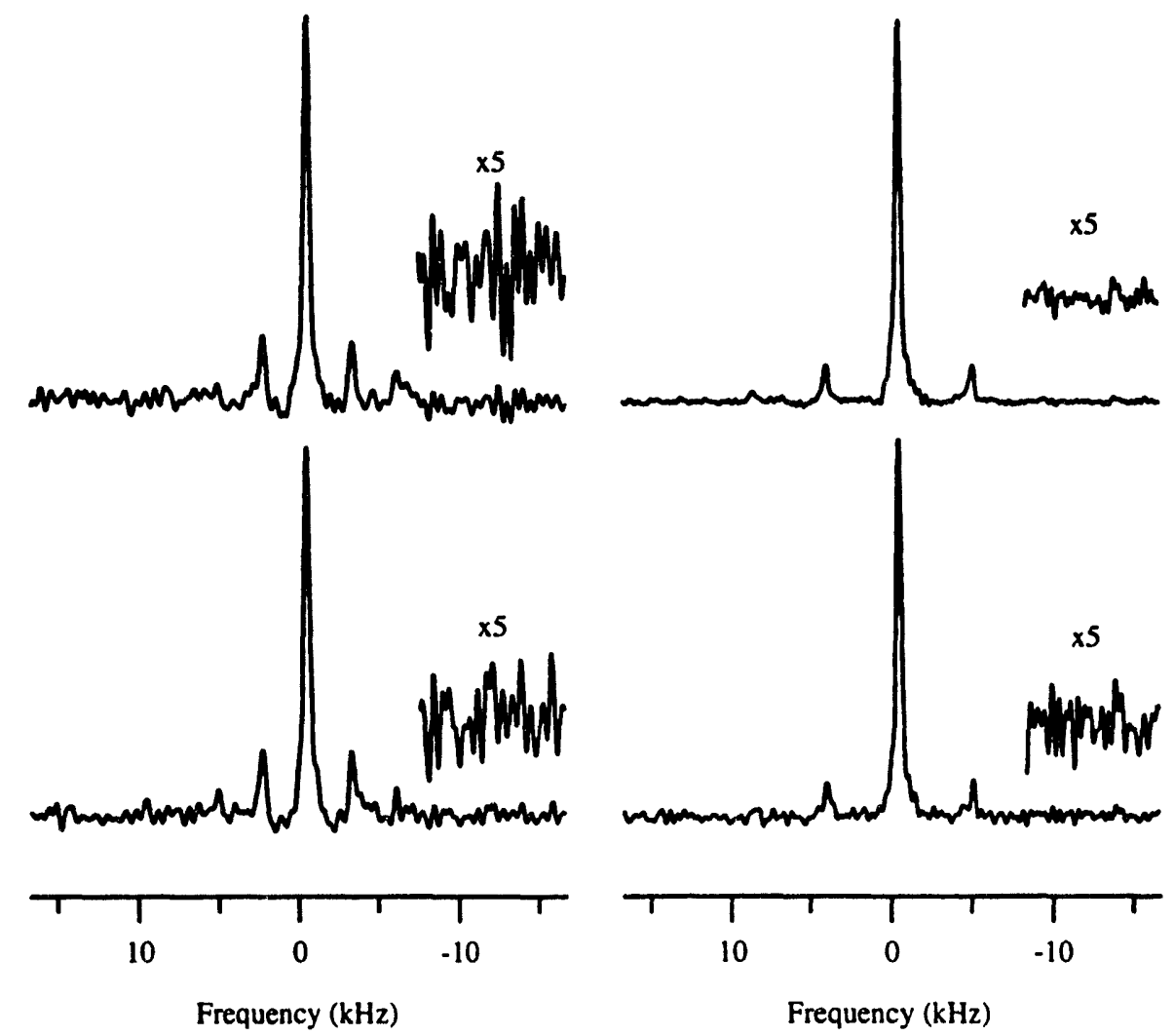

Figure 5.6 Comparison of CPDAS (fig 5.3e) versus DAS (fig 5.3d). The spectra on the right are $k=5$ DAS spectra while the spectra on the left are $k=1$. The upper spectra in both cases are from CPDAS.

without cross polarization. In fact, the CP efficiency under CPDAS at $k=1$ is very similar to that observed under VAS at $37.38^{\circ}$ (figure 5.2), which is to be expected. Other $k$ values will also have reduced $\mathrm{CP}$ efficiencies, in addition to having spinning sideband patterns which are more complicated than in the $k=1$ or 5 cases (see chapter 3). In table 5.1, the absolute signal-to-noise ratios for each the experiments in figure 5.6 are presented.

We have shown that the efficiency of $\mathrm{CP}$ is influenced very little by the choice of spinning angle until the angle approaches $0^{\circ}$. Therefore, in any VAS or DAS experiment 
it will be difficult to achieve maximum $\mathrm{CP}$ efficiency unless one does the magnetization transfer at $0^{\circ}$ as a part of, or before starting the experiment.

\begin{tabular}{lll}
\hline Experiment & Angle Pair & Signal to Noise Ratio \\
\hline CPDAS (fig 5.3e) & $\mathrm{k}=5\left(0.00^{\circ}, 63.43^{\circ}\right)$ & 123.6 \\
CPDAS (fig 5.3e) & $\mathrm{k}=1\left(37.38^{\circ}, 79.19^{\circ}\right)$ & 27.3 \\
Normal DAS (fig 5.3d) & $\mathrm{k}=5$ & 49.1 \\
Normal DAS (fig 5.36) & $\mathrm{k}=1$ & 32.3 \\
\hline
\end{tabular}

Table 5.1 Signal to Noise Ratios in CPDAS and Normal DAS

In addition, for the case of DAS the choice of $0^{\circ}$ and $63.43^{\circ}$ possesses the additional benefit of giving the largest effective spinning speed, $5 \omega_{r} / 6$, and narrowest residual homonuclear dipslar linewidth (see chapter 3 ). These results should prove quite valuable for systems with low abundance such as ${ }^{87} \mathrm{Rb}(27.8 \%$ abundant) or where isotopic labeling is crucial ${ }^{17} \mathrm{O}(0.037 \%$ abundant $)$ which could have polarization enhancements of 3.1 and 7.4 respectively leading to large savings in experiment time. In addition, for spin 1/2 systems (such as ${ }^{1} \mathrm{H}-{ }^{13} \mathrm{C}$ or ${ }^{1} \mathrm{H}_{-}{ }^{15} \mathrm{~N}$ ), where the time modulation of the dipolar interaction leads to modulations of the Hartmann-Hahn match condition, the use of ZPMAS may yield better CP efficiencies and reduced CPMAS distortions. 


\section{Chapter 6}

\section{Alternatives to DAS}

Throughout the preceding chapters both the theory and recent experimental advances of dynamic-angle spinning NMR have been discussed. This experiment was developed to produce high-resulution liquid-like spectra from solid samples containing quadrupolar nuclei such as ${ }^{17} \mathrm{O},{ }^{23} \mathrm{Na}$ and ${ }^{27} \mathrm{Al}$. Other techniques for averaging both the quadrupolar and chemical shift interactions have also been developed, including double rotation $^{55,108}$, magic-angle hopping ${ }^{109,110}$, magic-angle turning ${ }^{111}$ and dynamic-angle hopping. ${ }^{56}$

\section{Double Rotation (DOR)}

Double rotation is the simultaneous solution to the quadrupolar spinning problem in which at least two angles are required to give narrow isotropic spectra. .55

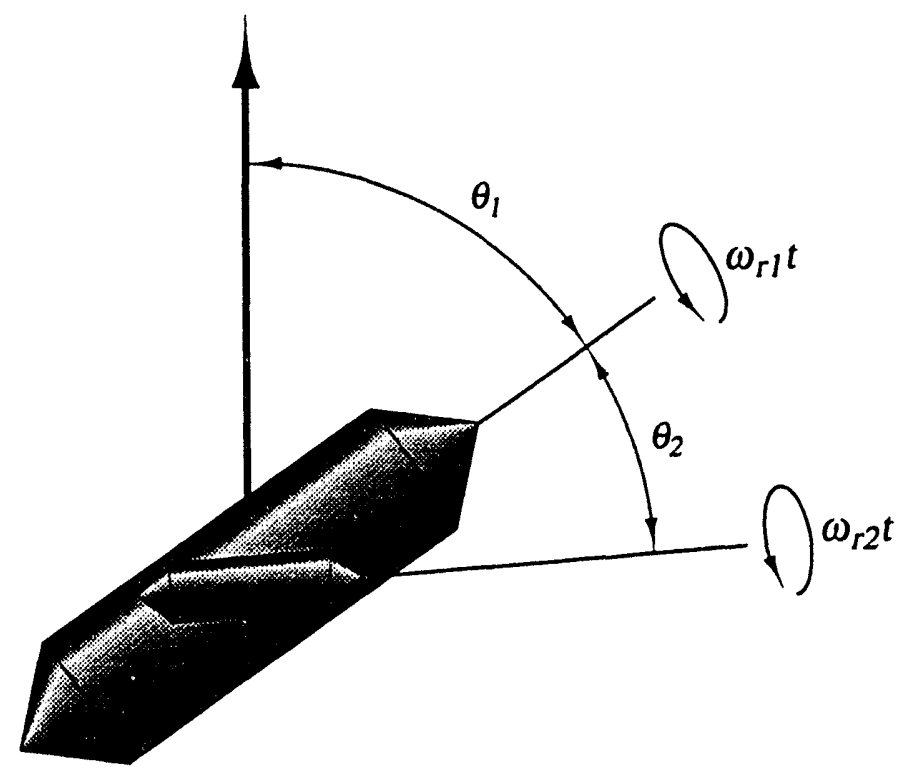

Figure 6.1 DOR Rotor \& Rotations. A representation of a DOR rotor is shown with the rotation angles given. These correspond to those shown in equation 6.1. The phase of the outer rotor is defined to be zero at zero time when the inner rotor makes the smallest angle with respect to the vertical axis (the magnetic field).

In this experiment, a small rotor is spun about an axis which slowly moves in a conical fashion about the magic-angle with respect to the magnetic field. Figure 6.1 shows the 
rotations needed to go from the PAS frame to the inner rotor frame then to the outer rotor frame and finally to the laboratory frame. The expression for the frequency under this time dependent trajectory is given below in equation 6.1.

$$
\begin{aligned}
\Delta E^{(2 Q)}= & \frac{n 2 \omega_{Q}^{2}}{\omega_{l}}\left(I(I+1)-\frac{3}{4}\right) \sum_{l=0,2,4} \sum_{m>0} \frac{\langle l, 0 \mid 2,2, m,-m\rangle}{m} a_{l 0}^{Q} \\
a_{l 0}^{Q}= & \sum_{n=-l}^{l} D_{n 0}^{(l)}\left(\omega_{r 1} t+\phi_{r 1}, \theta_{1}, 0\right) \sum_{j=-l}^{l} D_{j n}^{(l)}\left(\omega_{r 2} t+\phi_{r 2}, \theta_{2}, 0\right) \\
& \times \sum_{k=-l}^{l} D_{k j}^{(l)}\left(\alpha^{Q}, \beta^{Q}, \gamma^{Q}\right) \sigma_{l k}^{Q}
\end{aligned}
$$

In this expression, $\theta_{1}$ is the angle the outer rotation axis makes with respect to the magnetic field and $\theta_{2}$ is the angle the inner rotation axis makes with respect to the outer rotation axis. The outer rotation rate and absolute rotor phase are given by $\omega_{r 1}$ and $\phi_{1}$ while the inner rotation rate and absolute rotor phase are given by $\omega_{r 2}$ and $\phi_{2}$. The outer rotor phase is defined as zero when the inner rotation axis makes the smallest angle with respect to the magnetic field. The Euler angles refer to the rotation from the PAS to the inner rotor reference frames. Under the assumption of high speed spinning about both axes, this expression is greatly simplified.

$$
\begin{aligned}
\Delta E^{(2 Q)} & =\frac{\hbar 2 \omega_{Q}^{2}}{\omega_{l}}\left(I(I+1)-\frac{3}{4}\right) \sum_{l=0,2,4} \sum_{m>0} \frac{\langle l, 0 \mid 2,2, m,-m\rangle}{m} a_{l 0}^{Q} \\
a_{l 0}^{Q} & =d_{00}^{(l)}\left(\theta_{1}\right) d_{00}^{(l)}\left(\theta_{2}\right) \sum_{k=-l}^{l} e^{-i k \alpha^{Q}} D_{k 0}^{(l)}\left(\beta^{Q}\right) \sigma_{l k}^{Q}
\end{aligned}
$$


Much like the case of high speed magic angle spinning, the appearance of terms proportional to Legendre polynomials leads to the choice of DOR spinning angles. In the above expression, if $\theta_{2}$ is chosen equal to the root of the fourth-order Legendre polynomial $P_{4}\left[\cos \theta_{2}\right]$ and $\theta_{1}$ is chosen equal to the root of the second-order Legendre polynomial

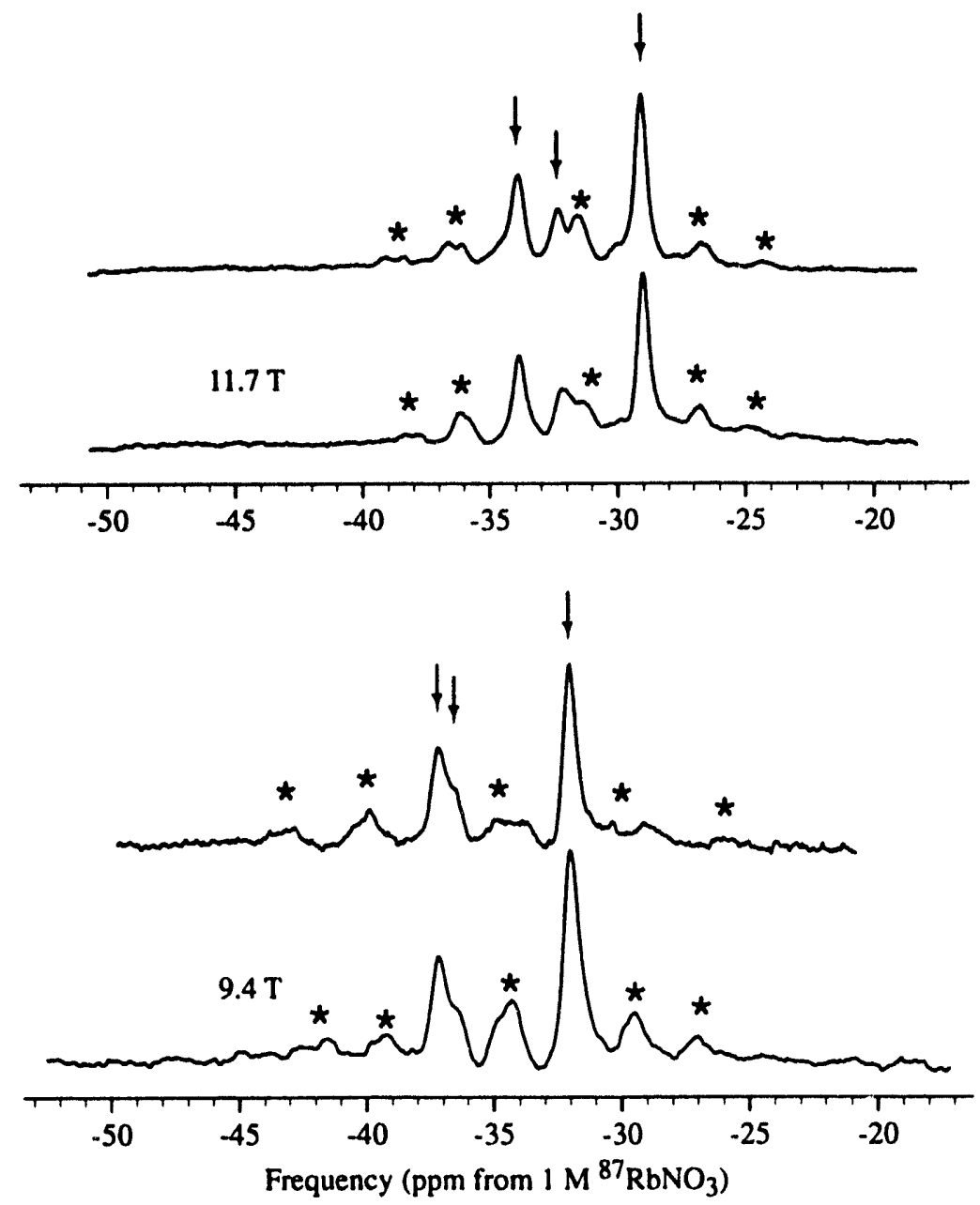

Figure 6.2 DOR of ${ }^{87} \mathrm{RbNO}_{3}$ at $9.4 \mathrm{~T}$. The two spectra above were taken with short $30^{\circ}$ pulses and a rapid repetition rate. This allowed acquisition of a large number of scans with random rotor phases, to achieve complete averaging of this variable leading to all positive sideband amplitudes (see below). The Larmor frequency at $11.7 \mathrm{~T}$ was 163.628 $\mathrm{MHz}$ and $130.886 \mathrm{MHz}$ at $9.4 \mathrm{~T}$. The spinning rates were $500 \mathrm{~Hz}$ (lower spectrum of each pair) and $700 \mathrm{~Hz}$ (uper spectrum) in these experiments.

$P_{2}\left[\cos \theta_{1}\right]$, only the $l=0$ terms will be non-zero. Alternatively, the angles may be reversed with the same effect. There is only one angle which is the root of $P_{2}\left[\cos \theta_{1}\right]$ which is the magic angle, $54.74^{\circ}$, while two angles are the roots of $P_{4}\left[\cos \theta_{2}\right], 30.56^{\circ}$ 
and $70.12^{\circ}$. Due to the requirements of constructing a DOR probshead, the usual choice is $54.74^{\circ}$ for the outer rotor and $30.56^{\circ}$ for the inner rotor. With these angles chosen, the DOR experiment is a simple single pulse and acquire experiment. Figure 6.2 shows the DOR spectra for ${ }^{87} \mathrm{RbNO}_{3}$, a salt with three crystallographically distinct sites. The spinning sidebands (marked with asterisks) arises from the time dependent terms which were ignored in equation 6.2. The isotropic peaks (marked with vertical arrows) are those which do not change position when the spinning rate is changed. These correspond to peaks at $-29,-32$ and $-34 \mathrm{ppm}$ at $11.7 \mathrm{~T}(163.628 \mathrm{MHz}$ Larmor frequency) and $-32,-36$ and -37 at $9.4 \mathrm{~T}$ (130.886 $\mathrm{MHz}$ Larmor frequency). The time dependent terms which lead to spinning sidebands may be analyzed in a manner virtually identical to that presented in chapter 3. First, we expand the energy splitting in equation 6.1 as the sum of oscillating time dependent terms.

$$
\begin{aligned}
\Delta E^{(2 Q)} & =\frac{\hbar 2 \omega_{Q}^{2}}{\omega_{l}}\left(I(I+1)-\frac{3}{4}\right) \sum_{l=0,2,4} \sum_{m>0} \frac{\langle l, 0 \mid 2,2, m,-m\rangle}{m} a_{l 0}^{Q} \\
a_{l 0}^{Q} & =\sum_{n=-l}^{l} \sum_{j=-l}^{l} \sum_{k=-l}^{l} d_{j n}^{(l)}\left(\theta_{2}\right) d_{n 0}^{(l)}\left(\theta_{1}\right) d_{k j}^{(l)}\left(\beta^{Q}\right) \sigma_{l k}^{Q} \\
& \times e^{-i\left[n\left(\omega_{r 1} l+\phi_{r 1}\right)+j\left(\omega_{r 2} l+\phi_{r 2}+\gamma^{Q}\right)+k \alpha^{Q}\right]}
\end{aligned}
$$

This expression may then be regrouped according to the dependence on rotation rates.

$$
\begin{aligned}
\Delta E^{(2 Q)} & =\sum_{l=0,2,4} \sum_{n, j=-l}^{l} W_{n j}^{(l)} e^{-i\left[n\left(\omega_{r 1} l+\phi_{r 1}\right)+j\left(\omega_{r 2} l+\phi_{r 2}+\gamma^{Q}\right)\right]} \\
W_{n j}^{(l)} & =\frac{\hbar 2 \omega_{Q}^{2}}{\omega_{l}}\left(I(I+1)-\frac{3}{4}\right) d_{j n}^{(l)}\left(\theta_{2}\right) d_{n 0}^{(l)}\left(\theta_{1}\right) \sum_{k=-l}^{l} d_{k j}^{(l)}\left(\beta^{Q}\right) \sigma_{l k}^{Q} \\
& \times e^{-i k \alpha^{Q}} \sum_{m>0} \frac{\langle l, 0 \mid 2,2, m,-m\rangle}{m}
\end{aligned}
$$

This may then be simplified by grouping the $l=0,2$, and 4 terms together for each $n, j$ pair. This simplifies equation 6.4 even further. 


$$
\begin{aligned}
\Delta E^{(2 Q)} & =\sum_{n, j=-4}^{4} W_{n j} e^{-i\left[n\left(\omega_{r 1} l+\phi_{r 1}\right)+j\left(\omega_{r 2} l+\phi_{r 2}+\gamma^{Q}\right)\right]} \\
W_{n j} & =\sum_{l=0,2,4} W_{n j}^{(l)}
\end{aligned}
$$

This may then be integrated to give the evolved phase and signal after a $90^{\circ}$ pulse.

$$
\begin{aligned}
\phi^{D O R}(t) & =W_{00 t}+\sum_{\substack{n, j=-4 \\
j=n \neq 0}}^{4} \frac{W_{n j} e^{-i\left[n\left(\omega_{r 1} t+\phi_{r 1}\right)+j\left(\omega_{r 2} t+\phi_{r 2}+\gamma^{Q}\right)\right.}}{-i\left(n \omega_{r 1}+j \omega_{r 2}\right)} \\
& +\sum_{\substack{n, j=-4 \\
j=n \neq 0}}^{4} \frac{-W_{n j} e^{-i\left[n \phi_{r 1}+j\left(\phi_{r 2}+\gamma^{Q}\right)\right]}}{-i\left(n \omega_{r 1}+j \omega_{r 2}\right)} \\
S(t)= & e^{-i \phi^{D O R}(t)}=e^{-i W_{00^{\ell}}} \\
& \times \exp \left[\sum_{\substack{n, j=-4 \\
j=n \neq 0}}^{4} \frac{\left.W_{n j} e^{-i\left[n\left(\omega_{r 1} t+\phi_{r 1}\right)+j\left(\omega_{r 2} t+\phi_{r 2}+\gamma^{Q}\right)\right.}\right]}{\left(n \omega_{r 1}+j \omega_{r 2}\right)}\right] \\
& \times \exp \left[\sum_{\substack{n, j=-4 \\
j=n \neq 0}}^{4} \frac{-W_{n j} e^{-i\left[n \phi_{r 1}+j\left(\phi_{r 2}+\gamma^{Q}\right)\right]}}{\left(n \omega_{r 1}+j \omega_{r 2}\right)}\right]
\end{aligned}
$$

The use of Dirac delta functions again may be used to simplify this equation.

$$
\begin{aligned}
S(t)= & e^{-i W_{00} t} \\
& \times \frac{1}{4 \pi^{2}} \int_{0}^{2 \pi} \int_{0}^{2 \pi}\left[x \exp \left[\sum_{\substack{n, j=-4 \\
j=n \neq 0}}^{4} \frac{W_{n j} e^{-i\left[n \psi+j \psi^{\prime}\right]}}{\left(n \omega_{r 1}+j \omega_{r 2}\right)}\right] d \psi d \psi^{\prime}\right] \\
& \times \frac{1}{4 \pi^{2}} \int_{0}^{2 \pi} \int_{0}^{2 \pi}\left[\times \exp \left[\sum_{\substack{n, j=-4 \\
j=n \neq 0}}^{4} \frac{-W_{n j} e^{-i\left[n \psi+j \psi^{\prime}\right]}}{\left(n \omega_{r 1}+j \omega_{r 2}\right)}\right] d \psi d \psi^{\prime}\right]
\end{aligned}
$$


Then substituting back the infinite sum expansion for the Dirac delta functions gives equation 6.8 .

$$
\begin{aligned}
S(t)= & e^{-i W_{00} t} \\
& \times \frac{1}{4 \pi^{2}} \sum_{N_{1}, N_{2}} \int_{0}^{2 \pi} \int_{0}^{2 \pi} \exp \left[\begin{array}{l}
i N_{1}\left(\psi-\omega_{r 1} t-\phi_{r 1}\right)+\sum_{\substack{n, j=-4 \\
j=n \neq 0}}^{4} \frac{W_{n j} j^{-i\left[n \psi+j \psi^{\prime}\right]}}{\left(n \omega_{r 1}+j \omega_{r 2}\right)} \\
+i N_{2}\left(\psi^{\prime}-\omega_{r 2} t-\phi_{r 2}-\gamma^{Q}\right)
\end{array}\right] d \psi d \psi^{\prime} \\
& \times \frac{1}{4 \pi^{2}} \sum_{N_{3}, N_{4}} \int_{0}^{2 \pi} \int_{0}^{2 \pi} \exp \left[\begin{array}{l}
i N_{3}\left(\psi-\phi_{r 1}\right)+\sum_{\substack{n, j=-4 \\
j=n \neq 0}}^{4} \frac{-W_{n j} e^{-i\left[n \psi+j \psi^{\prime}\right]}}{\left(n \omega_{r 1}+j \omega_{r 2}\right)} \\
+i N_{4}\left(\psi^{\prime}-\phi_{r 2}-\gamma^{Q}\right)
\end{array}\right] d \psi d \psi^{\prime}
\end{aligned}
$$

The $\psi$ and $\psi$ independent terms may be removed from the integrals and the signal may be expressed below.

$$
\begin{aligned}
S(t)= & e^{-i W_{00} t} \sum_{N_{1}, N_{2}, N_{3}, N_{4}} A_{N_{1}, N_{2}} A_{N_{3}, N_{4}}^{*} e^{-i\left[N_{1} \omega_{r 1}+N_{2} \omega_{r 2}\right] t} \\
& \times e^{-i\left[\left(N_{1}-N_{3}\right) \phi_{r 1}+\left(N_{2}-N_{4}\right)\left(\phi_{r 2}+\gamma^{Q}\right)\right]} \\
A_{N_{1}, N_{2}}= & \frac{1}{4 \pi^{2}} \int_{0}^{2 \pi} \int_{0}^{2 \pi} \exp \left[i N_{1} \psi+i N_{2} \psi^{\prime}+\sum_{\substack{n, j=-4 \\
j=n \neq 0}}^{4} \frac{W_{n j} e^{-i\left[n \psi+j \psi^{\prime}\right]}}{\left(n \omega_{r 1}+j \omega_{r 2}\right)}\right] d \psi d \psi^{\prime}
\end{aligned}
$$

This expression may then be integrated over the inner rotor phase $\left(N_{2}=N_{4}\right)$ due to the fact that usually we observe signal from powder samples (all $\gamma$ will be present).

$$
\langle S(t)\rangle_{\phi_{r 2}+\gamma^{Q}}=e^{-i W_{00} t} \sum_{N_{1}, N_{2}, N_{3}} A_{N_{1}, N_{2}} A_{N_{3}, N_{2}}^{*} e^{-i\left[N_{1} \omega_{r 1}+N_{2} \omega_{r 2}\right] t} e^{-i\left[\left(N_{1}-N_{3}\right) \phi_{r 1}\right]}
$$

The signal may then be averaged over all the powder angles and outer rotor orientations.

$$
\begin{aligned}
\langle S(t)\rangle_{\text {powder }} & =e^{-i \omega_{i s s}^{(2 Q)} t} \sum_{N_{1}, N_{2}} S_{N_{1}, N_{2}} e^{-i\left[N_{1} \omega_{r 1}+N_{2} \omega_{r 2}\right] t} \\
S_{N_{1}, N_{2}} & =\frac{1}{4 \pi} \int_{0}^{2 \pi} \int_{0}^{\pi}\left|A_{N_{1}, N_{2}}\right|^{2} \sin \beta^{Q} d \beta^{Q} d \alpha^{Q}
\end{aligned}
$$




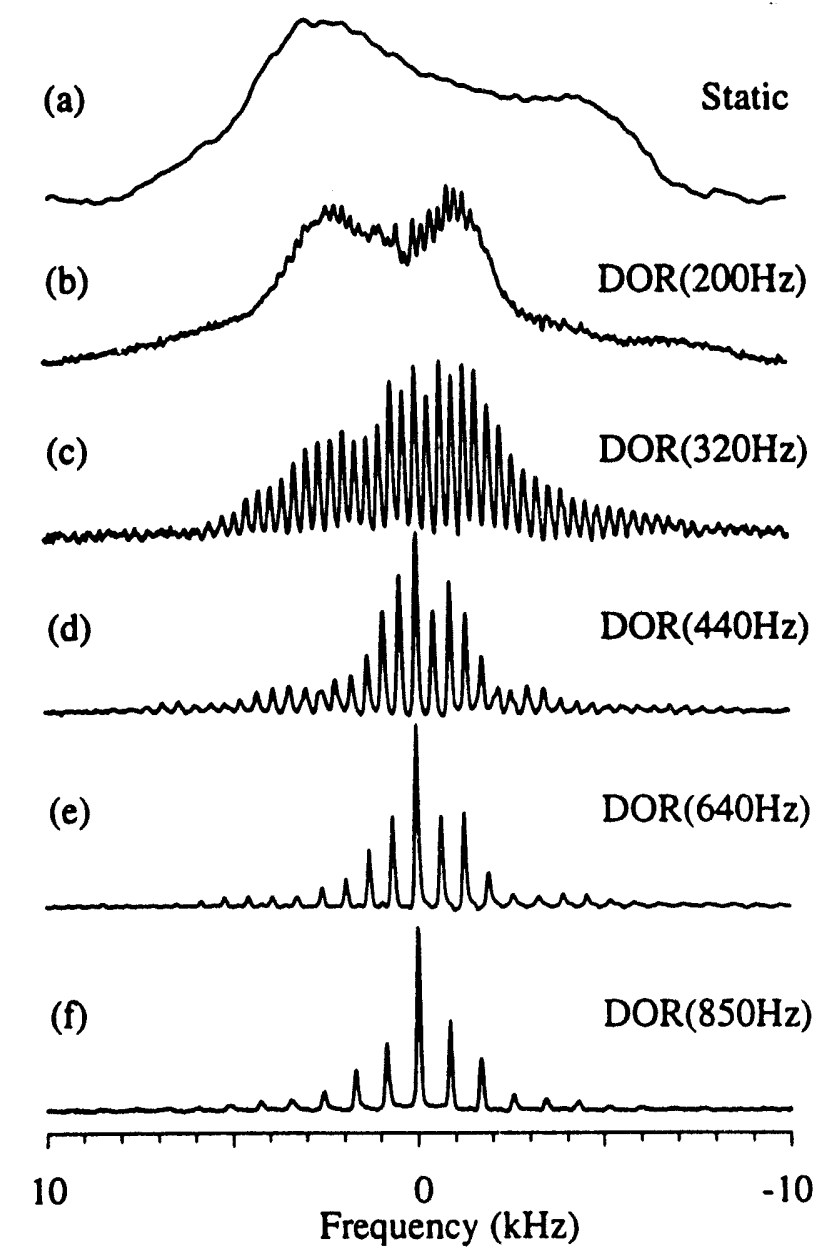

Figure 6.3 DOR of ${ }^{23} \mathrm{Na}_{2} \mathrm{C}_{2} \mathrm{O}_{4}$ at $9.4 \mathrm{~T}$. The spectra above were taken with the usual short pulses and a rapid repetition rate. Shown are the sideband intensities and positions for a variety of spinning rates (outer rotor spinning rate indicated beside each spectrum). It is important to note that the intensities do not necessarily approximate the powder pattern in the slow spinning limit.

This shows that spinning sidebands will show up at sums and differences of integers times the rotor frequencies. In general, the strongest sidebands will be those with small $N_{1}$ and $N_{2}$ values. For a more detailed analysis of DOR spinning sideband intensities, see papers by Sun et al. ${ }^{16.49}$ In figure 6.3 are shown the experimental DOR spectra of sodium oxalate at a variety of spinning rates. The most important feature here is that even in the slow spinning limit, the sideband intensities do not approximate the shape of the static pattern, as occurs in spin 1/2 systems under MAS. The spectra in figure 6.3 were taken by B.Q. Sun and Y. Wu and details concerning their acquisition may be found in their papers. ${ }^{16,49,112}$ 
In addition, certain symmetry considerations can lead to the cancellation of all of the odd-order outer frequency sidebands leading to greater sensitivity and resolution. $49,100,108,112-114$ To show this effect, we return to equation 6.6 and assume that the inner rotor is spinning much faster than the outer rotor and average over this motion.

$$
\begin{aligned}
\phi^{D O R}(t) & =W_{00} t+\sum_{n \neq 0} \frac{W_{n 0}\left(e^{-i n\left(\omega_{r 1} t+\phi_{r 1}\right)}-e^{-i n \phi_{r 1}}\right)}{-i n \omega_{r 1}} \\
S(t) & =e^{-i W_{00} t} \exp \left[\sum_{n \neq 0} \frac{W_{n 0}\left(e^{-i n\left(\omega_{r 1} t+\phi_{r 1}\right)}-e^{-i n \phi_{r 1}}\right)}{n \omega_{r 1}}\right]
\end{aligned}
$$

This, of course, now looks similar to the expression for the VAS signal in equation 3.11 or 3.21. There is one major difference, in that now the following substitution may be made for $W_{-n o}=-W_{n o}$. In fact, were this true for the VAS case, it would be possible to eliminate all odd order sidebands from any one dimensional experiment. This is not the case, however, and the rotor-synchronized acquisition described below will only give its effective speed enhancement under DOR conditions.

$$
S(t)=e^{-i W_{00} t} \exp \left[\sum_{n>0} \frac{W_{n 0}\left(\cos n\left(\omega_{r 1} t+\phi_{r 1}\right)-\cos n \phi_{r 1}\right)}{n \omega_{r 1}}\right]
$$

Signal may now be collected through outer-rotor synchronization such that the outer rotor phase is only $0^{\circ}$ or $180^{\circ}$. When this is done, the signal may be written below.

$$
\begin{aligned}
\langle S(t)\rangle^{0^{*}+180^{\circ}} & =e^{-i W_{00^{t}}} \exp \left[\sum_{n=2,4} \frac{W_{n 0}\left(\cos n \omega_{r 1} t-1\right)}{n \omega_{r 1}}\right] \\
& =e^{-i W_{00^{t}}} \exp \left[\sum_{n=1,2} \frac{W_{2 n 0}\left(\cos n \omega_{r 3} t-1\right)}{n \omega_{r 3}}\right] \\
\omega_{r 3} & =2 \omega_{r 1}
\end{aligned}
$$

Notice that a redefinition of the spinning rate has been made which changes the indices of the sum. This may be expanded with delta functions as before. 


$$
\begin{aligned}
\langle S(t)\rangle^{0^{\circ}+180^{\circ}}= & e^{-i W_{00^{t}}} \exp \left[\sum_{n=1,2} \frac{-W_{2 n 0}}{n \omega_{r 3}}\right] \\
& \times \frac{1}{2 \pi} \int_{0}^{2 \pi} \delta\left(\psi-\omega_{r 3} t\right) \exp \left[\sum_{n=1,2} \frac{W_{2 n 0} \cos n \psi}{n \omega_{r 3}}\right] d \psi
\end{aligned}
$$

Using the series expansion for a delta function we arrive at equation 6.16 .

$$
\begin{aligned}
\langle S(t)\rangle^{0^{\circ}+180^{\circ}} & =e^{-i W_{00} t} \exp \left[\sum_{n=1,2} \frac{-W_{2 n 0}}{n \omega_{r 3}}\right] \\
& \times \frac{1}{2 \pi} \sum_{N} \int_{0}^{2 \pi} \exp \left[i N\left(\psi-\omega_{r 3} t\right)+\sum_{n=1,2} \frac{W_{2 n 0} \cos n \psi}{n \omega_{r 3}}\right] d \psi
\end{aligned}
$$

Now we pull out constant terms from the integrals, just as in equation 3.14.

$$
\begin{aligned}
\langle S(t)\rangle^{0^{\circ}+180^{\circ}} & =e^{-i W_{00} t} \exp \left[\sum_{n=2,4} \frac{-W_{n 0}}{n \omega_{r 1}}\right] \sum_{N} A_{N} e^{-2 i N \omega_{r 1} t} \\
A_{N} & =\frac{1}{2 \pi} \int_{0}^{2 \pi} \exp \left[i N \psi+\sum_{n=2,4} \frac{W_{n 0} \cos (n \psi / 2)}{n \omega_{r 1}}\right] d \psi
\end{aligned}
$$

This signal may then be averaged over the remaining powder angles, giving the result in equation 6.18 .

$$
\begin{aligned}
\langle S(t)\rangle_{\text {powder }}^{0^{\circ}+180^{\circ}} & =\frac{1}{4 \pi} e^{-i \omega_{i s o}^{(2 Q)} t} \sum_{N} e^{-2 i N \omega_{r 1} t} \\
& \times \int_{0}^{2 \pi} \int_{0}^{\pi} \exp \left[\sum_{n=2,4} \frac{-W_{n 0}}{n \omega_{r 1}}\right] A_{N} \sin \beta^{Q} d \beta^{Q} d \alpha^{Q}
\end{aligned}
$$

This expression shows immediately that the sidebands will be spaced at twice the outer rotor spinning speed from the isotropic peak. This is quite useful, since it is difficult to spin a DOR outer rotor much faster than $1 \mathrm{kHz}$ and there will always be a large number of spinning sidebands present to complicate spectra. Figure 6.4 gives an example of the advantages of synchronized DOR when applied to the ${ }^{23} \mathrm{Na}$ spectrim of $\mathrm{Na}_{2} \mathrm{C}_{2} \mathrm{O}_{4}$. 

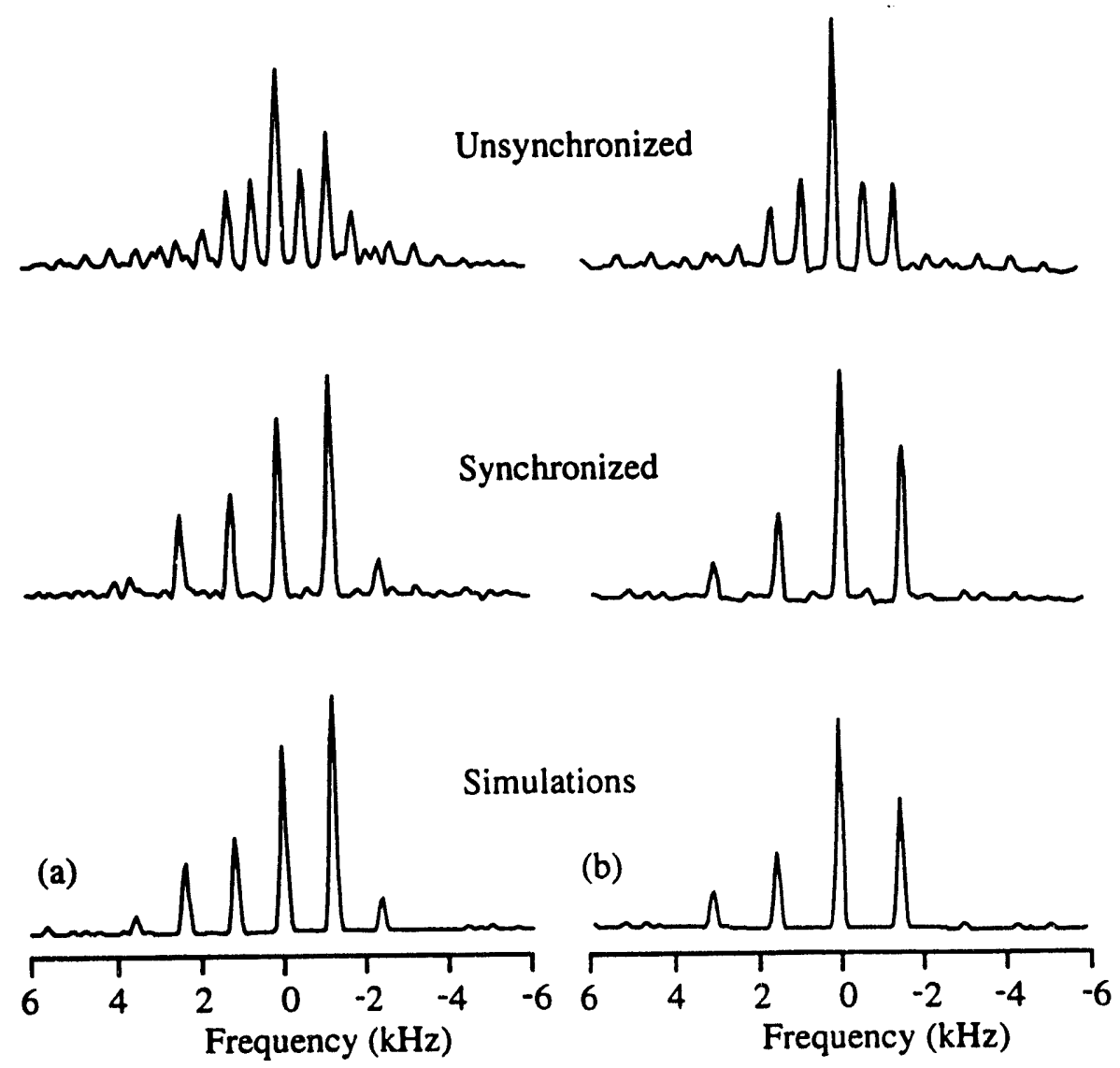

Figure 6.4 DOR of ${ }^{23} \mathrm{Na}_{2} \mathrm{C}_{2} \mathrm{O}_{4}$ at $9.4 \mathrm{~T}$. The spectra above were taken with short $30^{\circ}$ pulses and a rapid repetition rate. Absolute rotor phase was monitored using optical methods and pulses were applied only at the $0^{\circ}$ and $180^{\circ}$ positions. The outer rotor spinning rate was $604 \mathrm{~Hz}$ for the spectra on the left and $800 \mathrm{~Hz}$ for those on the right. For the simulations, the parameters were $C_{Q}=2.43 \mathrm{MHz}, \eta_{Q}=0.72$ and $\omega_{l}=105.8 \mathrm{MHz}$.

\section{Magic-Angle Hopping ( $\mathrm{MAH})$}

A different kind of experiment which generates isotropic spectra for spin 1/2 systems has been described by Bax et al. ${ }^{109}$ In this experiment, a static sample is allowed to evolve at three different orientations which define the vertices of an octahedron. This is accomplished by using $\mathrm{z}$-filters to store the evolved magnetization while the sample is rotated by 90 degrees about two orthogonal axes. The pulse sequence for this experiment is shown in figure 6.5 . 


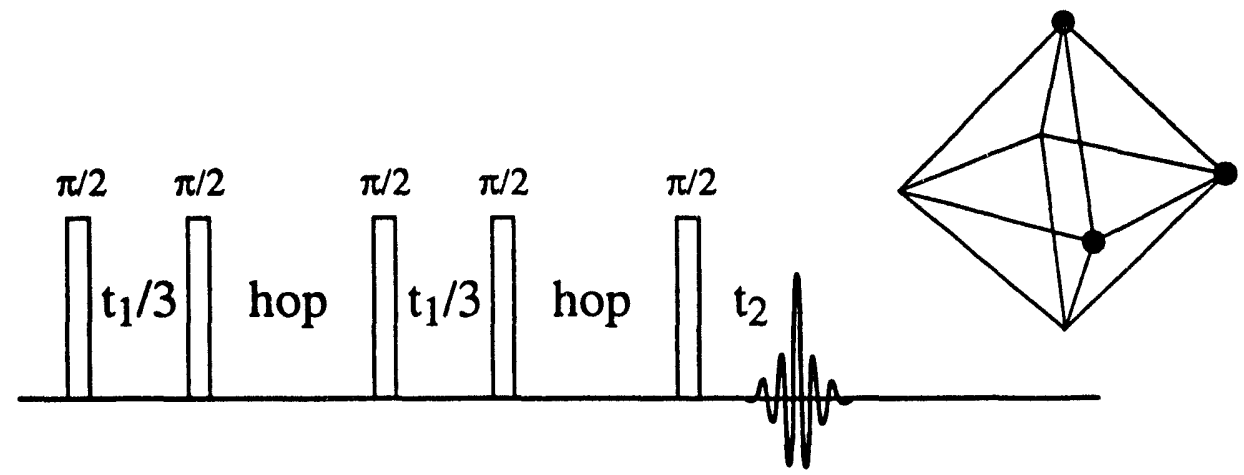

Figure 6.5 Magic-Angle Hopping Experiment. Pulses and hops are indicated schematically. The phase cycle is given in table 6.1. Each $t_{1} / 3$ period is spent with the magnetic field pointing through each of three vertices of an octahedron attached to the PAS of a given crystallite.

Alternatively, MAH may be accomplished by rotating the sample about the magic angle in three discrete $120^{\circ}$ jumps using the same pulse sequence. In any case, no spinning apparatus is required, however, the ability to perform rapid jumps may actually be of greater experimental complexity. Of these two implementations, the second is preferable, as it only requires rotation about a single axis.

\begin{tabular}{|cccc||cccc|}
\hline$\phi_{1}$ & $\phi_{2}, \phi_{4}, \phi_{5}$ & $\phi_{3}$ & $\phi_{\mathrm{r}}$ & $\phi_{1}$ & $\phi_{2}, \phi_{4}, \phi_{5}$ & $\phi_{3}$ & $\phi_{\mathrm{r}}$ \\
\hline 0 & 0 & 0 & 0 & 180 & 0 & 180 & 0 \\
0 & 0 & 90 & 90 & 180 & 0 & 270 & 90 \\
0 & 0 & 180 & 180 & 180 & 0 & 0 & 180 \\
0 & 0 & 270 & 270 & 180 & 0 & 90 & 270 \\
90 & 0 & 270 & 0 & 270 & 0 & 90 & 0 \\
90 & 0 & 0 & 90 & 270 & 0 & 180 & 90 \\
90 & 0 & 90 & 180 & 270 & 0 & 270 & 180 \\
90 & 0 & 180 & 270 & 270 & 0 & 0 & 270 \\
\hline
\end{tabular}

Table 6.1 Magic-Angle Hopping Experimental Phase Cycle. Phase cycle for MAH where the phase $\phi_{n}$ refers to the $n$th pulse in the pulse sequence. This same phase cycle may also be used for the MAT experiment (see figure 6.6).

The phase cycle needed to implement this experiment is given in table 6.1. Both the first and third pulses are cycled through four phases each to select $\Delta p=-1$. The $\Delta p=+1$ will 
be selected automatically without phase cycling the second or fourth pulses, since any non-zero coherences will decay during the hops. Finally, the last $\Delta p=-1$ will be selected by the quadrature phase of the receiver and merits no additional phase cycling, unless receiver quadrature is imperfect.

To show mathematically how the MAH evolution can generate an isotropic spectrum in the $t_{1}$ dimension of a two dimensional experiment we have to look at the frequency expression for the chemical shift interaction.

$$
\begin{aligned}
\omega_{C S A} & =\delta_{i s o, c s} \omega_{l}+\sqrt{\frac{2}{3}} \delta^{C S} \omega_{l} A_{2,0}^{C S} \\
A_{2,0}^{C S} & =\sum_{m=-2}^{2} D_{m, 0}^{(2)}\left(\alpha^{C S}, \beta^{C S}, \gamma^{C S}\right) \rho_{2, m}^{C S}
\end{aligned}
$$

The three Euler angles relate the laboratory frame to the principal axis frame of reference. In an experiment where the sample is rotated $\alpha^{\text {rot }}$ about an axis oriented $\beta^{\text {rot }}$ with respect to the magnetic field, this expression is modified as below.

$$
\begin{aligned}
& \omega_{C S A}=\delta_{i s o, c s} \omega_{l}+\sqrt{\frac{2}{3}} \delta^{C S} \omega_{l} \sum_{m=-2}^{2} D_{m, 0}^{(2)}\left(\alpha^{r o t}, \beta^{r o t}, 0\right) A_{2, m}^{C S} \\
& A_{2, m}^{C S}=\sum_{m^{\prime}=-2}^{2} D_{m^{\prime}, m}^{(2)}\left(\alpha^{C S}, \beta^{C S}, \gamma^{C S}\right) \rho_{2, m}^{C S}
\end{aligned}
$$

To examine the experiment where the sample is hopped in three 120 degree jumps about a given angle $\beta^{r o t}$ with respect to the magnetic field, we only have to sum up the evolution at each of the three rotor orientations. The three different orientations, expressed in the Euler angles are given below.

$$
\left(\alpha^{r o t}, \beta^{r o t}, 0\right) \Rightarrow\left\{\begin{array}{l}
\left(\alpha^{r o t}, \beta^{r o t}, 0\right) \\
\left(\alpha^{r o t}+\frac{2 \pi}{3}, \beta^{r o t}, 0\right) \\
\left(\alpha^{r o t}+\frac{4 \pi}{3}, \beta^{r o t}, 0\right)
\end{array}\right\}
$$

The net evolved phase over a period $t_{1}$ may then be written below 


$$
\left.\begin{array}{rl}
\phi^{M A H}\left(t_{1}\right) & =\delta_{i s o, c s} \omega_{l} t_{1} \\
& +\sqrt{\frac{2}{3}} \delta^{C S} \omega_{l} \frac{t_{1}}{3}\left[\sum_{m=-2}^{2}\left(\begin{array}{l}
D_{m, 0}^{(2)}\left(\alpha^{r o t}, \beta^{r o t}, 0\right)+ \\
D_{m, 0}^{(2)}\left(\alpha^{r o t}+\frac{2 \pi}{3}, \beta^{r o t}, 0\right)+ \\
D_{m, 0}^{(2)}\left(\alpha^{r o t}+\frac{4 \pi}{3}, \beta^{r o t}, 0\right)
\end{array}\right) A_{2, m}^{C S}\right.
\end{array}\right]
$$

The first term is the isotropic portion of the interaction which we wish to retain. The second is the anisotropic portion which will be shown to average to zero under the magicangle hopping experiment. The first simplification comes by setting $\beta^{r o t}$ to the magicangle $\left(54.74^{\circ}\right)$. This is the zero of the second-order Legendre polynomial and forces all of the $m=0$ terms to be zero. Secondly, by separating the Wigner rotation matrices into products of exponentials and reduced Wigner matrices via equation 6.23 , the sum in 6.22 may be further simplified.

$$
D_{m, 0}^{(2)}\left(\alpha^{r o t}, \beta^{r o t}, 0\right)=e^{-i m \alpha^{r o t}} d_{m, 0}^{(2)}\left(\beta^{r o t}\right)
$$

In this expression, the $m=0$ terms have been dropped as they are zero.

$$
\begin{aligned}
\phi^{M A H}\left(t_{1}\right) & =\delta_{i s o, c s} \omega_{l} t_{1}+\sqrt{\frac{2}{3}} \delta^{C S} \omega_{l} \frac{t_{1}}{3}\left[\sum_{m \neq 0} e^{-i m \alpha^{r o t}} d_{m, 0}^{(2)}\left(\beta^{r o t}\right) C_{m} A_{2, m}^{C S}\right] \\
C_{m} & =\left(1+e^{\frac{-2 i m \pi}{3}}+e^{\frac{-4 i m \pi}{3}}\right)
\end{aligned}
$$

The sum of exponentials $\left(C_{m}\right)$ inside the sum over $m$ is seen to be zero by using the following expressions.

$$
\sum_{i=1}^{n} \cos \frac{2 i \pi}{n}=\sum_{i=1}^{n} \sin \frac{2 i \pi}{n}=0 \quad \text { for } n>1
$$

This effectively removes all of the anisotropic contribution to the evolution and the net evolution and signal are given below.

$$
\begin{aligned}
\phi^{M A H}\left(t_{1}\right) & =\delta_{i s o, c s} \omega_{l} t_{1} \\
S\left(t_{1}\right) & =e^{-i \phi^{M A H}\left(t_{1}\right)}=e^{-i \delta_{i s o, c s} \omega_{l} l_{1}}
\end{aligned}
$$


An alternative approach to understanding this type of averaging scheme is to invoke group theoretical arguments as shown by Sun et al. ${ }^{16,113}$ This produces an identical result and will not be discussed here.

An excellent alternative to MAH is an experiment called magic-angle turning (MAT) first described by Gan. ${ }^{111}$ In this experiment, the sample is rotated continuously about the magic-angle, just as in MAS. However, now the sample is rotated at a very slow spinning speed (less than $100 \mathrm{~Hz}$ ). In this fashion, the evolution at each of the vertices of an octahedron may be approximated by interrupting the spinning with z-filters. This pulse sequence is seen in figure 6.6 below.

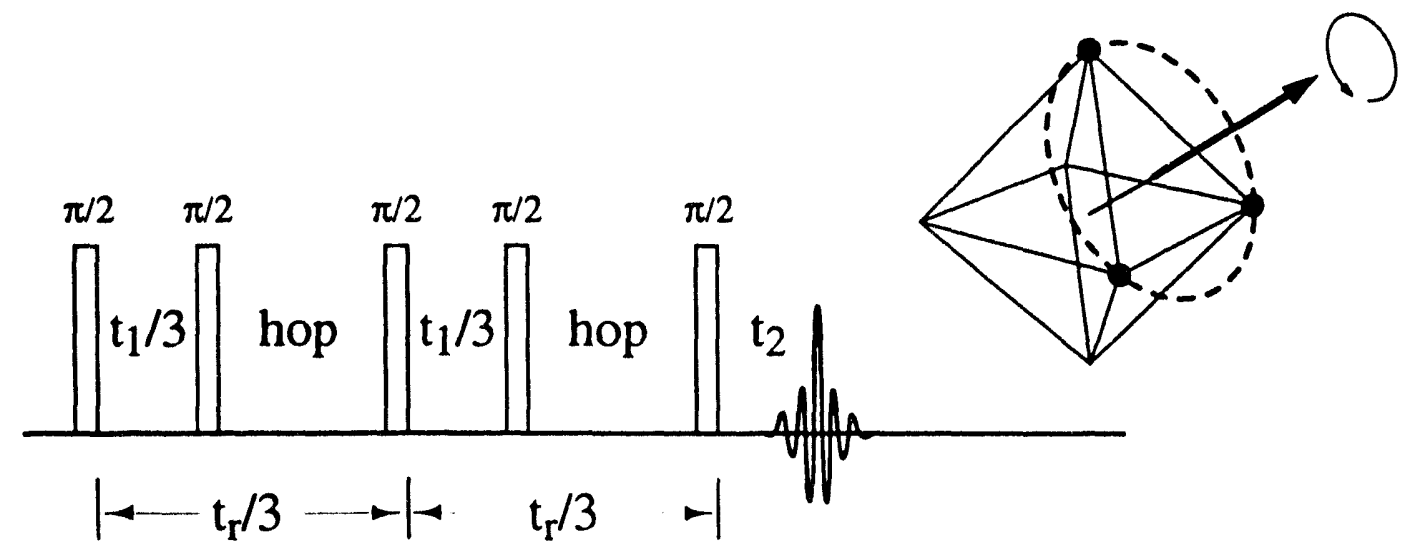

Figure 6.6 Magic-Angle Turning Experiment. Pulses and hops are indicated schematically. The phase cycle is the same as the MAH experiment (see table 6.1). Each hop is performed by allowing the rotor to shift by $120^{\circ}$ degrees. As in the previous experiment, each $t_{1} / 3$ period is spent with the magnetization at a different vertex of the octahedron, giving a shifting isotropic echo.

The theory for this experiment is identical in the limit of very slow spinning $\left(t_{1} \ll \tau_{r}\right)$. In the intermediate case, where $t_{1}$ represents is a significant portion of $\tau_{\mathrm{r}}$ (the period of the sample rotation), the theory must be written slightly differently. Now instead of the sum of three evolution periods, the frequency expression will be the sum of three integrals of the time-dependent frequencies. The expression for the NMR frequency of a sample rotating about an axis oriented at $\beta^{\text {rot }}$ with respect to the magnetic field as a function of both crystallite orientation $\left(\alpha^{C S}, \beta^{C S}, \gamma^{C S}\right)$ and time is given below. 


$$
\begin{aligned}
\omega_{C S A}(t) & =\delta_{i s o, c s} \omega_{l}+\sqrt{\frac{2}{3}} \delta^{C S} \omega_{l} R_{20}^{C S}(t) \\
R_{20}^{C S}(t) & =\sum_{m=-2}^{2} e^{-i m\left(\omega_{r} t+\phi_{r}\right)} d_{m 0}^{(2)}\left(\beta^{r o t}\right) A_{2 m}^{C S}
\end{aligned}
$$

In this expression the $A_{2 m}^{C S}$ term is identical to the $A_{2 m}^{C S}$ in equations 2.35 and 6.20. Now the net evolution following the MAT pulse sequence will be the sum of three integrals given below.

$$
\begin{aligned}
& \phi^{M A T}\left[t_{1}\right]= \delta_{\text {iso,cs }} \omega_{l} t_{1}+ \\
& \sqrt{\frac{2}{3}} \delta^{C S} \omega_{l}\left[\begin{array}{l}
\int_{0}^{\frac{t_{1}}{3}} R_{20}^{C S}(t) d t+\int_{\frac{\tau_{r}}{3}}^{\frac{\tau_{r}+t_{1}}{3}} R_{20}^{C S}(t) d t \\
+\int_{\frac{2 \tau_{r}}{3}}^{\frac{2 \tau_{r}+t_{1}}{3}} R_{20}^{C S}(t) d t
\end{array}\right]
\end{aligned}
$$

Again, the first term is the isotropic chemical shift and the second corresponds to the anisotropic parts. The integrals themselves are over sums which can be separated into a larger sum of integrals. The time-independent part is proportional to the second-order Legendre polynomial of $\cos \beta^{r o t}$. This is analytically zero, since we have chosen $\beta^{\text {rot }}$ to be the magic-angle, $54.74^{\circ}$, which means the sum in equation 6.29 will contain no $m=0$ terms. Each of the integrals may performed analytically and regrouped below.

$$
\begin{aligned}
& \phi^{M A T}\left[t_{1}\right]=\delta_{i s o, c s} \omega_{l} t_{1} \\
& +\sqrt{\frac{2}{3}} \delta^{C S} \omega_{l} \sum_{m=-2}^{2} \frac{e^{-i m \phi_{r}}}{i m \omega_{r}} d_{m o}^{(2)}\left(\beta^{r o t}\right) A_{2 m}^{C S}\left[\begin{array}{l}
\exp \left(\frac{-i m \omega_{r} t_{1}}{3}\right) \exp (0)-\exp (0) \\
+\exp \left(\frac{-i m \omega_{r} t_{1}}{3}\right) \exp \left(\frac{-i m \omega_{r} \tau_{r}}{3}\right)-\exp \left(\frac{-i m \omega_{r} \tau_{r}}{3}\right) \\
+\exp \left(\frac{-i m \omega_{r} t_{1}}{3}\right) \exp \left(\frac{-i m \omega_{r} 2 \tau_{r}}{3}\right)-\exp \left(\frac{-i m \omega_{r} 2 \tau_{r}}{3}\right)
\end{array}\right]
\end{aligned}
$$

These may be further simplified since $\omega_{r} \tau_{r}=2 \pi$.

$$
\begin{aligned}
& \phi^{M A T}\left[t_{1}\right]=\delta_{i s o, c s} \omega_{l} t_{1} \\
& +\sqrt{\frac{2}{3}} \delta^{C S} \omega_{l} \sum_{m=-2}^{2} \frac{e^{-i m \phi_{r}}}{i m \omega_{r}} d_{m o}^{(2)}\left(\beta^{r o t}\right) A_{2 m}^{C S}\left[\exp \left(\frac{-i m \omega_{r} t_{1}}{3}\right) C_{m}-C_{m}\right]
\end{aligned}
$$

The $C_{m}$ in this expression is identical to that in 6.24. Using the fact that $C_{m}$ is zero, yields the same frequency expression as in equation 6.26 . 


$$
\begin{aligned}
& \phi^{M A T}\left[t_{1}\right]=\delta_{i s o, c s} \omega_{l} t_{1} \\
& S\left(t_{1}\right)=e^{-i \phi^{M A T}\left[t_{1}\right]}=e^{-i \delta_{i s o, c s} \omega_{l} t_{1}}
\end{aligned}
$$

Therefore an anisotropic echo in $t_{2}$ will appear at a time $t_{1} / 3$ following the last pulse in the MAT sequence which has evolved in $t_{1}$ with an isotropic frequency. In both the MAH and MAT experiments, however, signal is lost due to the z-filters. In fact, if the zfilters could be eliminated, a factor of 2 signal-to-noise could be recovered.

A possible alternative to the MAH and MAT sequences is the $\mathrm{MAT}^{56}$ with $\pi$ pulses (MAT-180) sequence which is shown in figure 6.7.

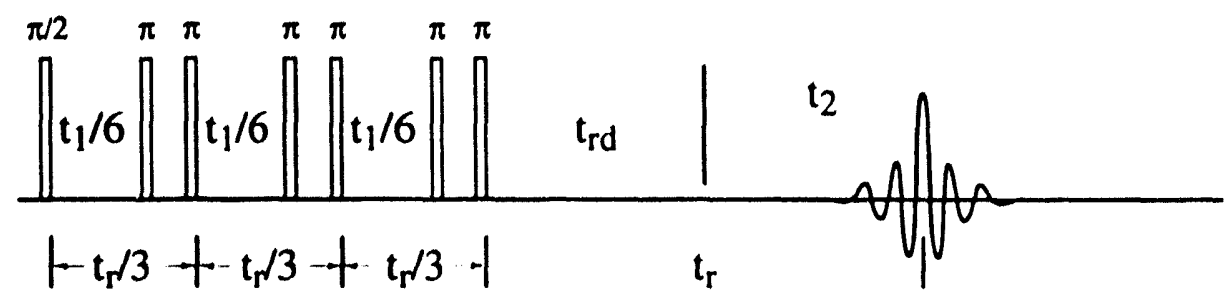

Figure 6.7 Magic-Angle Turning Experiment with $\pi$ Pulses. Pulses are indicated schematically. The phase cycle is given in the text below. In this experiment, no storage pulses are used while rotor shifts by $120^{\circ}$. Each of the $t_{1} / 6$ periods is spent at a different vertex of the octahedron, giving a shifting isotropic echo.

In this experiment, the density matrix is never stored with $z$-filters. However, now the sequence has been made into a constant time experiment (as $t_{1}$ is varied, the MAT isotropic echo will always appear at a point $t_{r}$ after the last $\pi$ pulse) which introduces certain other problems which I will discuss later. The phase cycle needed to implement this experiment is quite simple, assuming the $\pi$ pulses are accurate. Only the first pulse is cycled through four phases and the receiver phase is set equal to this phase (just as in a standard one pulse experiment with cyclops phase cycling.) To show mathematically why this experiment works, we use the same approach as earlier. The phase is expressed below in equation 6.32 . 


$$
\begin{aligned}
\phi^{M A T-180}\left[t_{r}\right] & =\int_{0}^{\frac{t_{1}}{6}} \omega_{C S A}(t) d t-\int_{\frac{t_{1}}{6}}^{\frac{t_{r}}{3}} \omega_{C S A}(t) d t \\
& +\int_{\frac{t_{r}}{3}}^{\frac{t_{1}}{6}+\frac{t_{r}}{3}} \omega_{C S A}(t) d t-\int_{\frac{t^{2}}{6}+\frac{t_{r}}{3}}^{\frac{2 t_{r}}{3}} \omega_{C S A}(t) d t \\
& +\int_{\frac{t_{r}}{3}}^{\frac{t_{1}}{6}+\frac{2 t_{r}}{3}} \omega_{C S A}(t) d t-\int_{\frac{t_{r}}{6}+\frac{2 t_{r}}{3}}^{t_{C S A}} \omega_{C S A}(t) d t+\int_{t_{r}}^{2 t_{r}} \omega_{C S A}(t) d t
\end{aligned}
$$

The integral from $\tau_{\mathrm{r}}$ to $2 \tau_{\mathrm{r}}$ in this sum may be divided into six integrals with the same limits as the corresponding terms in 6.32 and since $\omega_{C S A}(t)=\omega_{C S A}\left(t+t_{r}\right)$, the negative terms will cancel terms from the expansion of the last integral. The expression for the CSA frequency may be substituted in 6.32 and the time-independent terms removed from the integrals as in 6.27 and 6.28 .

$$
\phi^{M A T-180}\left[t_{r}\right]=\delta_{i s o, c s} \omega_{l} t_{1}+\sqrt{\frac{2}{3}} \delta^{C S} \omega_{l}\left[\begin{array}{l}
2 \int_{0}^{\frac{t_{1}}{6}} R_{20}^{C S}(t) d t+2 \int_{\frac{\tau_{r}}{3}}^{\frac{2 \tau_{r}+t_{1}}{6}} R_{20}^{C S}(t) d t \\
+2 \int_{\frac{2 \tau_{r}}{3}}^{\frac{4 \tau_{r}+t_{1}}{6}} R_{20}^{C S}(t) d t
\end{array}\right]
$$

This integration may be performed as earlier, yielding the same phase as in the MAH and MAT experiments.

$$
\begin{aligned}
& \phi^{M A T-180}\left[t_{r}\right]=\delta_{i s o, c s} \omega_{l} t_{1} \\
& S\left(t_{1}\right)=e^{-i \phi^{M A T-180}\left[t_{r}\right]}=e^{-i \delta_{i s o, c s} \omega_{l} t_{1}}
\end{aligned}
$$

This shows that all three experiments give the identical result. The difference between the MAT-180 and the MAT experiment lies in the sensitivity and resolution. In the MAT experiment, the resolution is improved by taking more $t_{1}$ points with a corresponding longer total $2 t_{\mathrm{r}}$ time. The sensitivity, however, is a full factor 2 worse than the MAT-180 sequence (meaning a factor of 4 more scans are needed). In the case of MAT-180, to enhance the resolution, the rotor must be slowed down (since the longest available $t_{1}$ point is for $t_{1}=2 t_{r}$ ). Since this is a constant-time experiment, there will be more transverse $\left(T_{2}\right)$ relaxation for the same $t_{1}$ point at slower speeds than at faster speeds since each $t_{1}$ point has identical $2 t_{r}$ transverse relaxation scaling the overall intensity. At some point, 
in attempting to gain further resolution in a MAT- 180 experiment, the relaxation intensity loss will become larger than the factor of 2 loss due to z-filters in the MAT or MAH experiments. At this point, it is more profitable to use the MAH or MAT experiment in favor of the MAT-180 sequence. Finally, since the MAT-180 sequence is constant time, there should be no net homonuclear dipolar contribution to a spin-1/2 isotropic spectrum (as is the case for the MAT sequence). In addition, both the MAT and MAT-180 may be performed over more than one or two rotor cycles.

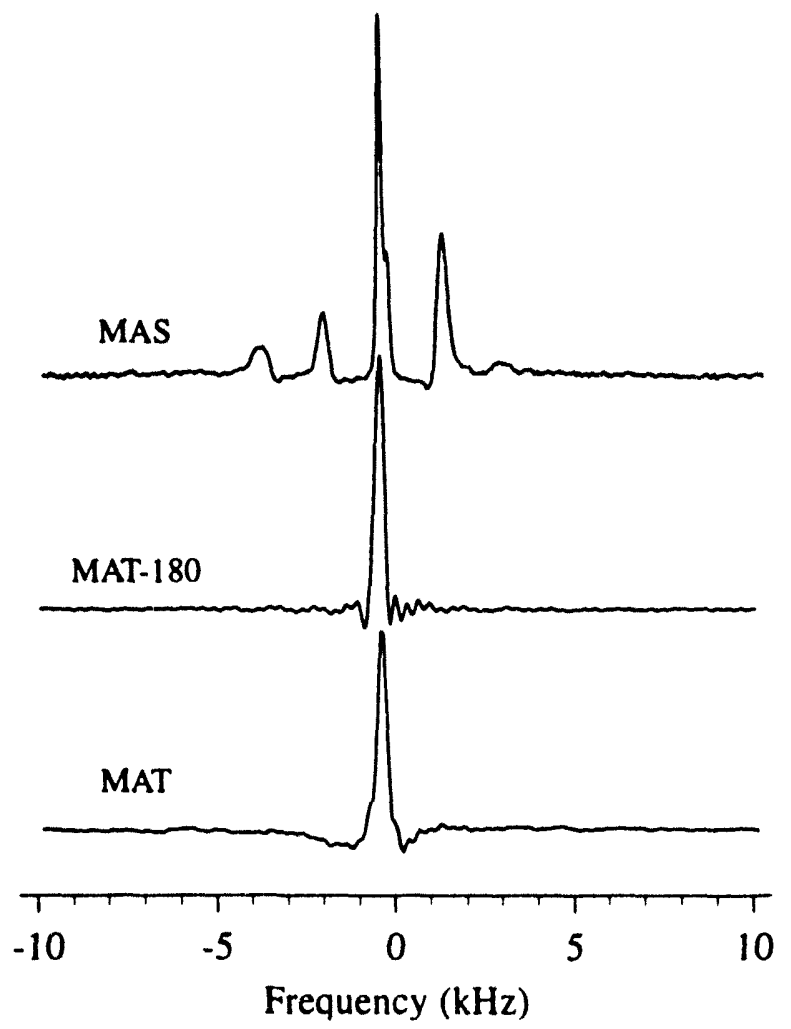

Figure 6.8 MAS, MAT and MAT-180 Spectra of ${ }^{207} \mathrm{PbNO}_{3}$. All of these spectra represent 64 points in $t_{1}$ zero filled to 512 . The dwell times were $50 \mu$ s and the $90^{\circ}$ pulse widths were $12 \mu \mathrm{s}$.

In the case of MAT, any number of rotor cycles may be used which is not a multiple of three, while for MAT-180, any even number of rotor cycles which is not a multiple of three may be used. (If the number of rotor cycles is a multiple of three, each of the three evolution periods will have identical starting phase and no averaging will result.) Figure 6.8 shows the MAS, MAT and MAT -180 spectra of ${ }^{207} \mathrm{PbNO}_{3}$. Notice, in this case, the 
signal-to-noise ratio of the MAT-180 spectrum is slightly better than in the MAT spectrum, since the $2 t_{r}$ time was chosen to be relatively short. This has the adverse effect of adding truncation artifacts to the spectrum in the form of "sinc" wiggles.

\section{Dynamic-Angle Hopping (DAH)}

In the previous section on magic-angle hopping methods were discussed which produce sideband-free isotropic spectra. In the case of spin-1/2 nuclei, a number of techniques are already available which produce sideband free evolution. ${ }^{115-122}$ The application of these methods to central transitions of quadrupolar nuclei is somewhat limited, especially in the case where sidebands overlap centerband features. The ideas of MAH, however, may be applied equally well to the quadrupolar problem (DAH). ${ }^{56,113}$ In the quadrupolar case the integrals of equation 6.29 will involve a sum from $m=-4$ to +4 . The additional $m= \pm 3$ and \pm 4 terms will cause the simple MAH and MAT experiments to fail, since the value of $C_{m} \neq 0$ for $m= \pm 3$ and \pm 4 . To average these as well, five different evolution windows are needed (in the case of hopping about the magic-angle). In this case, the expression for $C_{m}$ is given below (which is zero for all $m<5$ ).

$$
C_{m}=\left(1+e^{\frac{-2 i m \pi}{5}}+e^{\frac{-4 i m \pi}{5}}+e^{\frac{-6 i m \pi}{5}}+e^{\frac{-8 i m \pi}{5}}\right)
$$

This is still not sufficient to produce isotropic spectra, since five hops about the magicangle will only give a sideband free MAS spectrum (not altogether useless). To isotropically average a quadrupolar central transition, it has been shown that multiple spinning

axes are required. ${ }^{16,42,43.55,113}$ The solution to the problem is to use two DAS angles and use five evolution periods at each angle to cancel the time dependent terms. This is a total of 10 evolution periods, needing a minimum of 9 z-filters to store magnetization during hops. This is almost certainly an unacceptable number and therefore a better solution is to choose the DAS angle pair which simplifies the problem. The $k=5$ angle pair of $63.43^{\circ}$ and $0.00^{\circ}$ is the best angle pair for this sort of experiment, since at $0.00^{\circ}$ there are 
no time-dependent terms. Thus, to do the DAH experiment, we merely spin slowly about the angle $63.43^{\circ}$ with respect to the magnetic field. Under these conditions we use $90^{\circ}$ pulses to store the evolution between the five $72^{\circ}$ jumps. Following these five evolution periods, the magnetization is stored as the spinning axis is realigned to $0.00^{\circ}$ and evolution is allowed to proceed again. This experiment still needs a total of $5 \mathrm{z}$-filters for magnetization storage, but this represents a factor of 4 improvement in signal-to-noise over the $9 \mathrm{z}$-filter experiment proposed earlier (unfortunately it is still a factor of 4 worse than in a conventional DAS experiment).

The phase cycle needed to implement this DAH experiment is quite long, since a large number of pulses are involved in the sequence. The schematic pulse sequence and the equation which describes the relationship between the eleven $90^{\circ}$ pulses and the receiver phases are given below. The time $172^{\circ}$ indicates the time needed to allow the rotor to rotate $72^{\circ}$ and the time thop indicates the time needed to reorient the spinning axis from $63.43^{\circ}$ to $0.00^{\circ}$.

$$
\begin{aligned}
& 90^{\circ}-\frac{t_{1}}{6}-90^{\circ}-t_{72^{\circ}}-90^{\circ}-\frac{t_{1}}{6}-90^{\circ}-t_{72^{\circ}}-90^{\circ}-\frac{t_{1}}{6}- \\
& 90^{\circ}-t_{72^{\circ}}-90^{\circ}-\frac{t_{1}}{6}-90^{\circ}-t_{72^{\circ}}-90^{\circ}-\frac{t_{1}}{6}-90^{\circ}-t_{\text {hop }}-90^{\circ}-\frac{t_{1}}{6}-t_{2} \\
& -\phi_{1}+\phi_{2}-\phi_{3}+\phi_{4}-\phi_{5}+\phi_{6}-\phi_{7}+\phi_{8}-\phi_{9}+\phi_{10}-\phi_{11}=-\phi_{R}
\end{aligned}
$$

In this experiment, the coherence alternates between -1 (during the $t_{1} / 6$ evolution periods) and 0 (during the $t 72^{\circ} \mathrm{z}$-filter storage periods). To achieve this, the first pulse should be cycled through four phases to choose the -1 coherence transfer and the second will be uncycled, assuming that the $72^{\circ}$ hopping period will be sufficiently long that all transverse magnetization will decay away. This, in theory, should be continued for each of the next 8 pulse pairs. The last pulse may be left uncycled, since the receiver quadrature will select the -1 pathway. This is a total of $4^{5}$, or 1024 , steps in the phase cycle. In many situations, this is too many steps to do a phase cycle over for a spectrometer (i.e. the Bruker $^{\mathrm{TM}}$ AM-400) or more scans than is possible in a two-dimensional experiment due to 
long relaxation times (remember that the isotropic DAH signal must be collected point by point in $t_{1}$ just as in DAS). One solution is to use only cycles of pulse phases of three rather than four. This will still choose only a $\Delta p=-1$, however this may be difficult to implement on some spectrometers. Also, it still requires a total $3^{5}$, or 243 , steps which is over a factor of four fewer scans. In the case where fewer scans are desired, it is necessary to choose fewer pulses to cycle. It is probably best to cycle pulses closer to the beginning of the sequence, thereby guaranteeing the coherence pathway for most of the early steps. This can lead to experimental artifacts. Ideally, we should cycle the other pulses (except the last) through two steps to guarantee no transverse components during z-filters. This will expand the phase cycle again by a factor of $2^{5}$ (a total factor of 32 ).

A final note about the sequence is that the five angles at $63.43^{\circ}$ and one at $0.00^{\circ}$ are equivalent to the static magnetic field being rotated to point through the vertices of an icosohedron (which has the symmetry needed to average first and second order interactions) in the PAS coordinate system.

A second implementation of the DAH experiment is to use $180^{\circ}$ pulses (DAH180 ), just as in the MAT-180 sequence. Instead of storing the magnetization with $z$-filters, we can instead apply rotor synchronized $180^{\circ}$ pulses in the following sequence, where the time variables have the same meaning as before.

$$
\begin{gathered}
90^{\circ}-t_{r}-\frac{t_{1}}{12}-180^{\circ}-t_{72^{\circ}}-180^{\circ}-\frac{t_{1}}{12}-180^{\circ}-t_{72^{\circ}}-180^{\circ}-\frac{t_{1}}{12}-180^{\circ}-t_{72^{\circ}}-180^{\circ} \\
-\frac{t_{1}}{12}-180^{\circ}-t_{72^{\circ}}-180^{\circ}-\frac{t_{1}}{12}-180^{\circ}-t_{72^{\circ}}-90^{\circ}-t_{\text {hop }}-90^{\circ}-\frac{t_{1}}{6}-t_{2} \\
-\phi_{1}+2 \phi_{2}-2 \phi_{3}+2 \phi_{4}-2 \phi_{5}+2 \phi_{6}- \\
2 \phi_{7}+2 \phi_{8}-2 \phi_{9}+2 \phi_{10}-\phi_{11}-\phi_{12}=-\phi_{R}
\end{gathered}
$$

The phase cycle needed to implement DAH-180 is much simpler than for DAH, assuming the $180^{\circ}$ pulses are accurate and lead to only a \pm 2 coherence transfer. In this case, only the first pulse needs to be cycled through four phases and the eleventh through two (just as in the original DAS experiment). In fact, the sequence is identical to the original 
DAS experiment with the addition of nine $180^{\circ}$ pulses. These may be ignored in this case for phase cycling purposes. Any of the pure-phase modifications discussed in chapter 4 may be used here to enhance the overall signal-to-noise in the two-dimensional spectrum (if the we are interested in an isotropic/anisotropic correlation spectrum). Again, both the DAH and DAH-180 cycles may be implemented over more than two rotor cycles, just as in the case of MAT and MAT-180. Since the frequency expansion has sines and cosines up to $4 \omega_{r}$, the number of evolution periods must always be larger than this (we choose 5), and the number of rotor periods the experiment is performed over must not be a multiple of the number of evolution periods (in this case 5). The mathematics needed to prove these features for the DAH and DAH-180 are identical to the case of MAT and MAT-180. These types of experiments have recently been discussed by Gann et al. ${ }^{56}$ and Alderman et al. ${ }^{123}$ and I would direct the interested reader to these papers for additional information.

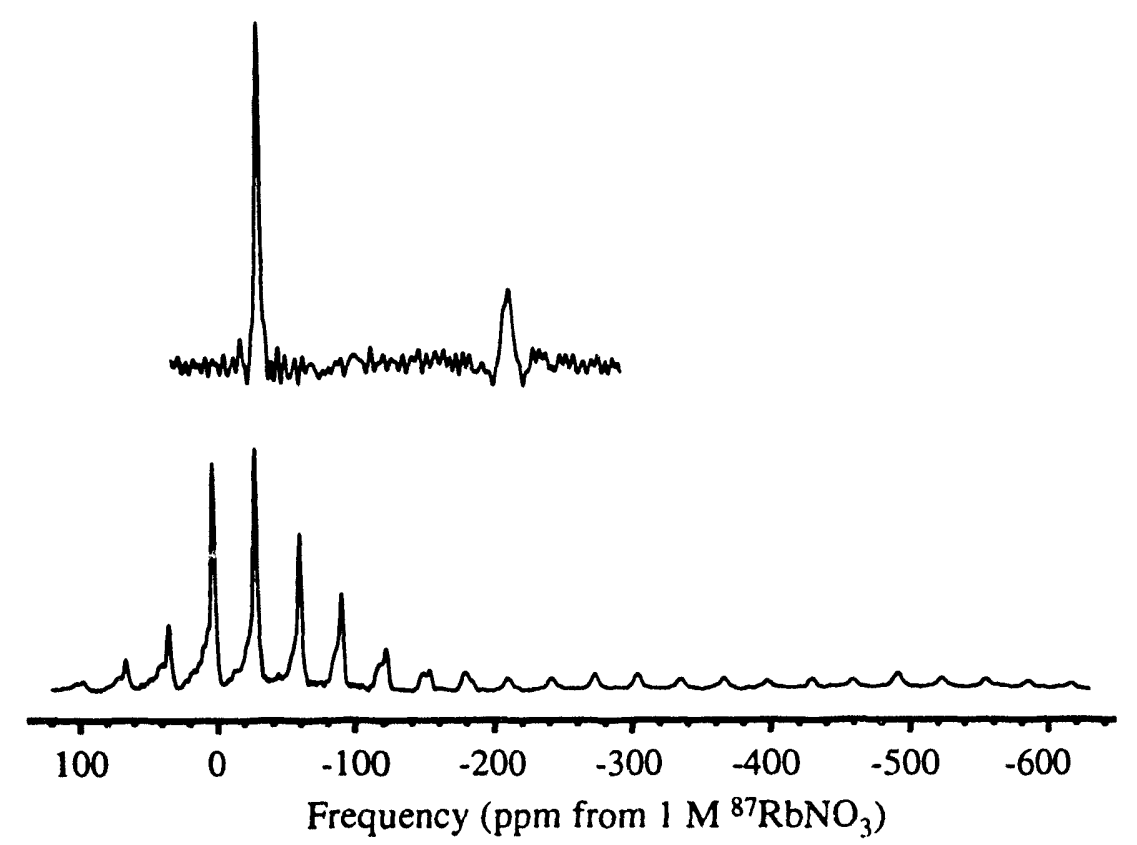

Figure 6.9 DAS and DAH ID spectra of ${ }^{87} \mathrm{Rb}_{2} \mathrm{CrO}_{4}$. The upper DAH spectrum shows no spinning sidebands and the isotropic peaks are easily identified, while in the lower DAS spectrum the broad site with an isotropic shift at $-201 \mathrm{ppm}$ breaks into a large number of sidebands. A second spinning speed would be needed to identify this as the isotropic site using this method. 
Figure 6.9 shows the DAS and DAH spectra for rubidium chromate $\left({ }^{87} \mathrm{Rb}_{2} \mathrm{CrO}_{4}\right)$ taken at 9.4 $\mathrm{T}$ which has two sites. Both sites appear in the DAS spectrum, however the broad site with an isotropic shift at $-201 \mathrm{ppm}$ is greatly reduced in intensity due to the large number of spinning sidebands. Even the more intense peak at $-27 \mathrm{ppm}$ has at least six spinning sidebands in this spectrum. The appearance of the isotropic peak at -201 ppm in the DAH spectrum shows the power of the DAH experiment. This peak is much more intense than the same peak in the DAS spectrum. This peak, unfortunately, is broadened more than the peak at $-27 \mathrm{ppm}$, thereby making the intensity seem much less than the expected $1: 1$ ratio. This is probably due to angle errors during the $72^{\circ}$ hops while spinning at $63.43^{\circ}$ which result from fluctuations in the spinning rate. Finally, because of the large number of $z$-filters, the DAH experiment required over 20 times the number of scans and therefore 20 times the overall experiment time as the comparable DAS experiment. This factor negates much of the benefit of DAH, since the same information may be attained with just two DAS experiments in a tenth the time.

In figure 6.10 we see the DAS and DAH-180 spectra for rubidium sulfate $\left({ }^{87} \mathrm{Rb}_{2} \mathrm{SO}_{4}\right)$ at $9.4 \mathrm{~T}$ which also has two sites. Both sites appear in both spectra. However, in the DAH-180 spectrum, there are no spinning sidebands to complicate the interpretation. Also, the intensities of the two peaks should reflect the populat: 1 at the two sites (which is $1: 1$ in this case). For the DAS spectrum, intensity measurement necessitates integration of a large number of spinning sidebands (some of which overlap). If we compare only the heights of the isotropic centerbands, we arrive at a ratio of 2.8 ($25 \mathrm{ppm}$ site) to 8.5 (29 ppm site). This 1:3 ratio is much less than expected from the crystal structure. Taking the heights of each sideband as the integral and adding up the intensity for each of the sidebands in the DAS spectrum, yields intensities of 9.2 (-25 ppm site) and 10.1 (29 ppm site). These are, as expected, quite close to the 1:1 ratio, however, in samples with multiple sites, integration of sideband intensity may be impos- 
sible. For the DAH-180 spectrum, we may easily integrate each of the two peaks (2.62 and 2.34 intensities respectively) and get the correct $1: 1$ ratio.

(b)
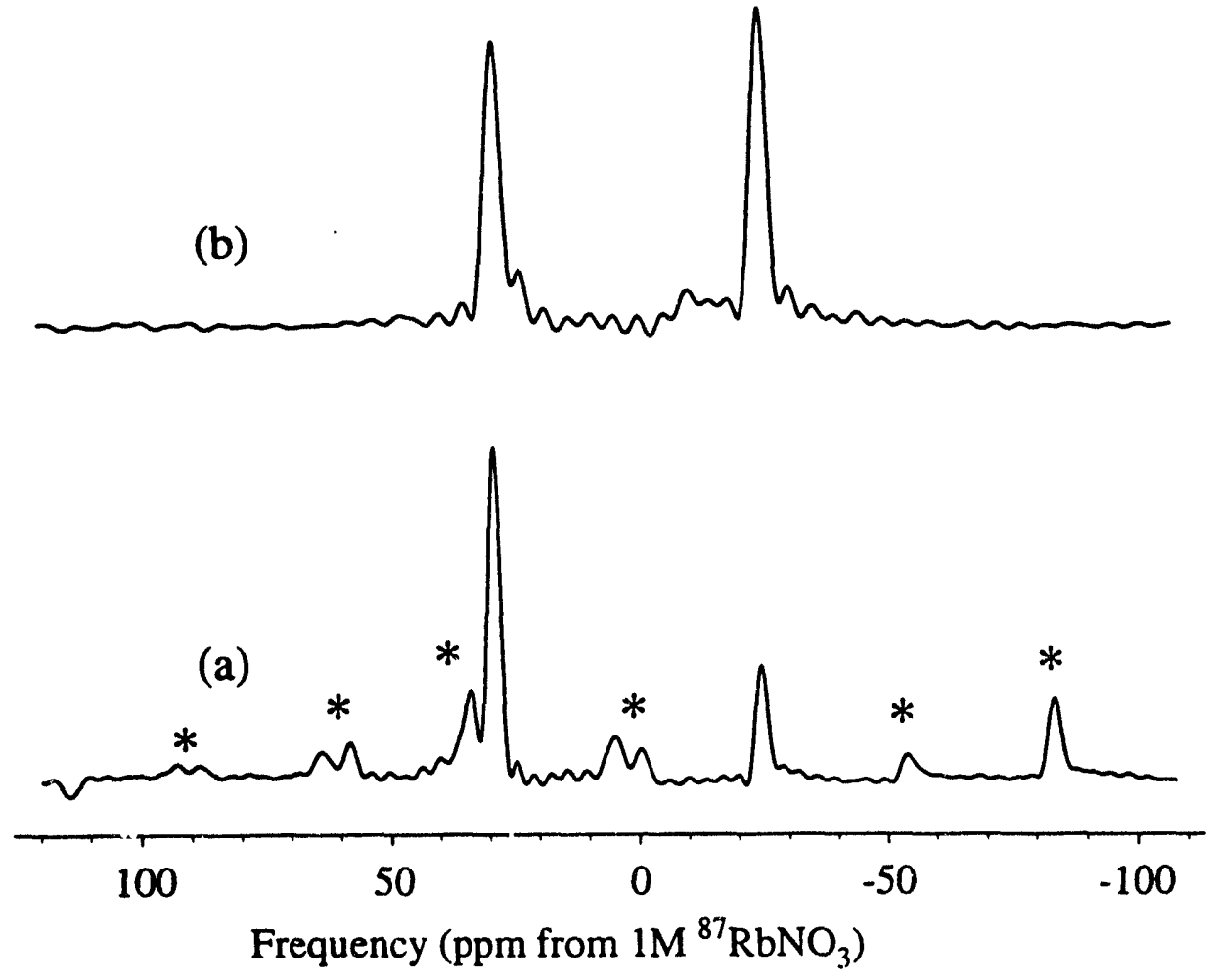

Figure 6.10 DAS and DAH-180 1D spectra of ${ }^{87} \mathrm{Rb}_{2} \mathrm{SO}_{4}$;. Spectrum (a) shows the $9.4 \mathrm{~T}$ DAS spectrum of ${ }^{87} \mathrm{Rb}_{2} \mathrm{SO}_{4}$ taken at a spinning rate of $5 \mathrm{kHz}$ and with the SEDAS pulse sequence. Spinning sidebands are indicated with asterisks. Spectrum (b) shows the 9.4T DAH-180 spectrum of the same compound taken with the $9 \pi$ pulse sequence applied over 8 rotor cycles at $2.4 \mathrm{kHz}$.

As was mentioned earlier for the MAT-180 sequence, constant-time experiments may sometimes present sensitivity problems when additional resolution is needed. The DAH-180 sequence is partially a constant-time experiment since $5 / 6$ of the $t_{1}$ evolution occurs under constant time conditions. Therefore, as can be seen in figure 6.10 , we often get truncation artifacts in DAH-180 spectra. These sequences, however, show great promise for studying systems where an inhomogeneous distribution of isotropic shifts exists, for example in an amorphous solid such as a glasse (see chapter 8). In these cases, the distribution of isotopic shifts leads to a rapid dephasing in $t_{1}$ (in fact, much more rapid than the intrinsic $T_{2}$ linewidth would suggest). This means that many fewer point 
are required in $t_{1}$ before the signal disappears. Therefore a constant time experiment such as DAH-180 is uniquely well suited for the study of these systems, just as pulse sequences such as HyperSEDAS are best suited for giving high-sensitivity pure-phase twodimensional spectra. Combining these two ideas should greatly improve the overall quality of isotropic/anisotropic correlation spectra in amorphous solids. 


\section{Chapter 7}

\section{Application of DAS to Inorganic Salts}

Dynamic-angle spinning has proven quite valuable in the evaluation of the electric field gradients present at the nuclei in a variety of inorganic salts. Specifically, the alkali metals are particularly conducive for study with NMR. All of these nuclei are spin-3/2 and possess a manageable quadrupolar moment. In the case of ${ }^{7} \mathrm{Li}$ and ${ }^{133} \mathrm{Cs}$, the quadrupolar interactions are generally small enough that MAS is sufficient to achieve high resolution spectra. The nuclei of ${ }^{87} \mathrm{Rb},{ }^{85} \mathrm{Rb}$ and ${ }^{23} \mathrm{Na}$, however, require the more complete averaging of a technique like DAS or DOR.

\section{Sodium}

This nucleus was one of the first evaluated with DAS. The large gyromagnetic ratio and high natural abundance make this a natural candidate for study with DAS. However, these two factors conspire to make the homonuclear dipolar interaction quite strong and therefore highest resolution is only achieved in samples where the sodium is magnetically diluted, for example in crown ether complexes or with bulky anions in ionic salts. Unfortunately, the total chemical shift range for this nucleus is quite small, as is the range of electric field gradients, since this is a fairly small cation. Therefore, the overall linewidth from the homonuclear dipolar coupling (see chapter 3 ) renders DAS insensitive to small variations in the local EFG and chemical shift interaction. Examples of sodium spectra are shown in both chapter 3 and chapter 5 .

\section{Rubidium Salts}

The application of dynamic-angles spinning NMR has also been extended to other inorganic salts. ${ }^{54}$ Specifically, ${ }^{87} \mathrm{Rb}$ has proven to be an extremely sensitive nucleus for DAS experiments. ${ }^{87} \mathrm{Rb}$ and alkali metals in general are important in a number of areas, 
they serve as promoters in catalysts, for example, the heterogeneous catalysis of ammonia synthesis ${ }^{124}$ and oxidative coupling of methane to yield ethane and ethene. ${ }^{125}$ Rubidium is an important component of some glasses, ${ }^{126}$ and recently, it has been shown that Buckminsterfullerene, $\mathrm{C}_{60}$, doped with $\mathrm{Rb}$ metal becomes superconducting at $28 \mathrm{~K}^{127}$

In order to assess the applicability of DAS to rubidium and its potential to yield structural information about materials such as those listed above, ${ }^{87} \mathrm{Rb}$ MAS, VAS and DAS spectra of five inorganic salts were obtained. The salts chosen were $\mathrm{RbCl}, \mathrm{RbClO}_{4}$, $\mathrm{Rb}_{2} \mathrm{SO}_{4}, \mathrm{Rb}_{2} \mathrm{CrO}_{4}$ and $\mathrm{RbNO}_{3}$ because they had been previously studied with static NMR experiments ${ }^{128.129}$ and the crystal structures were well known. ${ }^{130-135}$

In the study by Cheng et al., ${ }^{128}$ the $T_{1}$ relaxation times for each of these salts was measured and all were between 100 and $300 \mathrm{~ms}$. We performed the VAS, MAS, and DAS experiments using a probe designed by Mueller et al. ${ }^{51}$ We used the usual DAS pulse sequences (see chapters 3 and 4 ) for both the ID spectra ${ }^{42}$ and for the pure-phase MAS detected spectra. ${ }^{50}$ Our central transition selective $90^{\circ}$ pulses were between 4.0 and $6.0 \mu \mathrm{s}$. Our hopping times were usually between 30 and $50 \mathrm{~ms}$ and our data sets were $128 \mathrm{t}_{1}$ points by 512 complex $t_{2}$ points. All MAS and variable-angle spinning (VAS) spectra were acquired with a standard Hahn-echo pulse sequence $\left(\pi / 2-n t_{r}-\pi-t_{r d}-\right.$ acquire) where $t_{r}$ is the rotor period, $n$ is an integer and $n t_{r}$ was between 500 and $1500 \mu \mathrm{s}$. The $t_{r d}$ delay was used to allow collection of the whole echo.

For ${ }^{87} \mathrm{Rb}$, as with all quadrupolar nuclei, the measured isotropic shift in DAS has a field dependence because it is the sum of two contributions.

$$
\delta_{o b s}=\delta_{i s o, c s}+\delta_{i s o}^{(2 Q)}
$$

These two terms are the isotropic chemical shift and the isotropic second-order quadrupolar shift, respectively. The isotropic chemical shift is field independent when expressed in units of ppm while the second-order quadrupolar shift has a strong field dependence given below. 


$$
\delta_{i s o}^{(2)}=-\frac{3 \times 10^{6}}{40} \frac{C_{Q}{ }^{2}\left(I(I+1)-\frac{3}{4}\right)\left(1+\frac{\eta_{Q}^{2}}{3}\right)}{\omega_{l}^{2} I^{2}(2 I-1)^{2}}
$$

All constants have their usual meanings. Using equations 7.1 and 7.2 , we may calculate isotropic chemical shifts by measuring isotropic shifts at two field strengths. To do this, we solve the system of linear equations from equation 7.3 evaluated at two $B_{0}$ fields. Equation 7.3 (the reduced version of equation 7.1) may be expressed as follows for ${ }^{87} \mathrm{Rb}$ in units of ppm.

$$
\begin{aligned}
\delta_{o b s} & =\delta_{i s o, c s}-\left(1.28 \times 10^{-10} \frac{\mathrm{T}^{2}}{\mathrm{~Hz}^{2}}\right) C_{Q}^{2}\left(1+\frac{\eta_{Q}^{2}}{3}\right)\left(\frac{1}{B_{0}^{2}}\right) \\
& =\delta_{i s o, c s}-\left(1.28 \times 10^{-10} \frac{\mathrm{T}^{2}}{\mathrm{~Hz}^{2}}\right) P_{Q}{ }^{2}\left(\frac{1}{B_{0}^{2}}\right) \\
P_{Q} & \equiv C_{Q} \sqrt{1+\frac{\eta_{Q}^{2}}{3}}
\end{aligned}
$$

It is important to note that it is impossible to extract the $C_{Q}$ from $\eta_{Q}$ using only multiple field experimental ID DAS results. However, multiple field results do help to minimize experimental error in the final results.

Figure 7.1 shows the ${ }^{87} \mathrm{Rb}$ VAS spectra and the angles of acquisition. Only the $\mathrm{RbCl}$ is clearly resolved. This is because of the absence of quadrupolar coupling due to the cubic crystal structure of $\mathrm{RbCl}$. Figures 7.2 and 7.3 show the DAS spectra at 11.7T and 9.4T respectively. There is an order of magnitude narrowing of the DAS spectra compared to the VAS spectra. In the cases where multiple lines are present due to spinning sidebands, the isotropic peaks were identified by spinning at multiple spinning rates.

The VAS spectra in figure 7.1 show the resolving power of simple one-dimensional NMR techniques applied to Rb salts. In all cases except $\mathrm{RbNO}_{3}$ and $\mathrm{Rb}_{2} \mathrm{CrO}_{4}$, the individual sites are clearly separated. However, only the $\mathrm{RbCl}$ spectrum yields a single narrow line which may be used to measure the isotropic shift. The other spectra would all require simulations to extract the actual isotropic shifts, and in the case of $\mathrm{RbNO}_{3}$, the simulation would be quite difficult due to the extreme overlap of the three sites. 


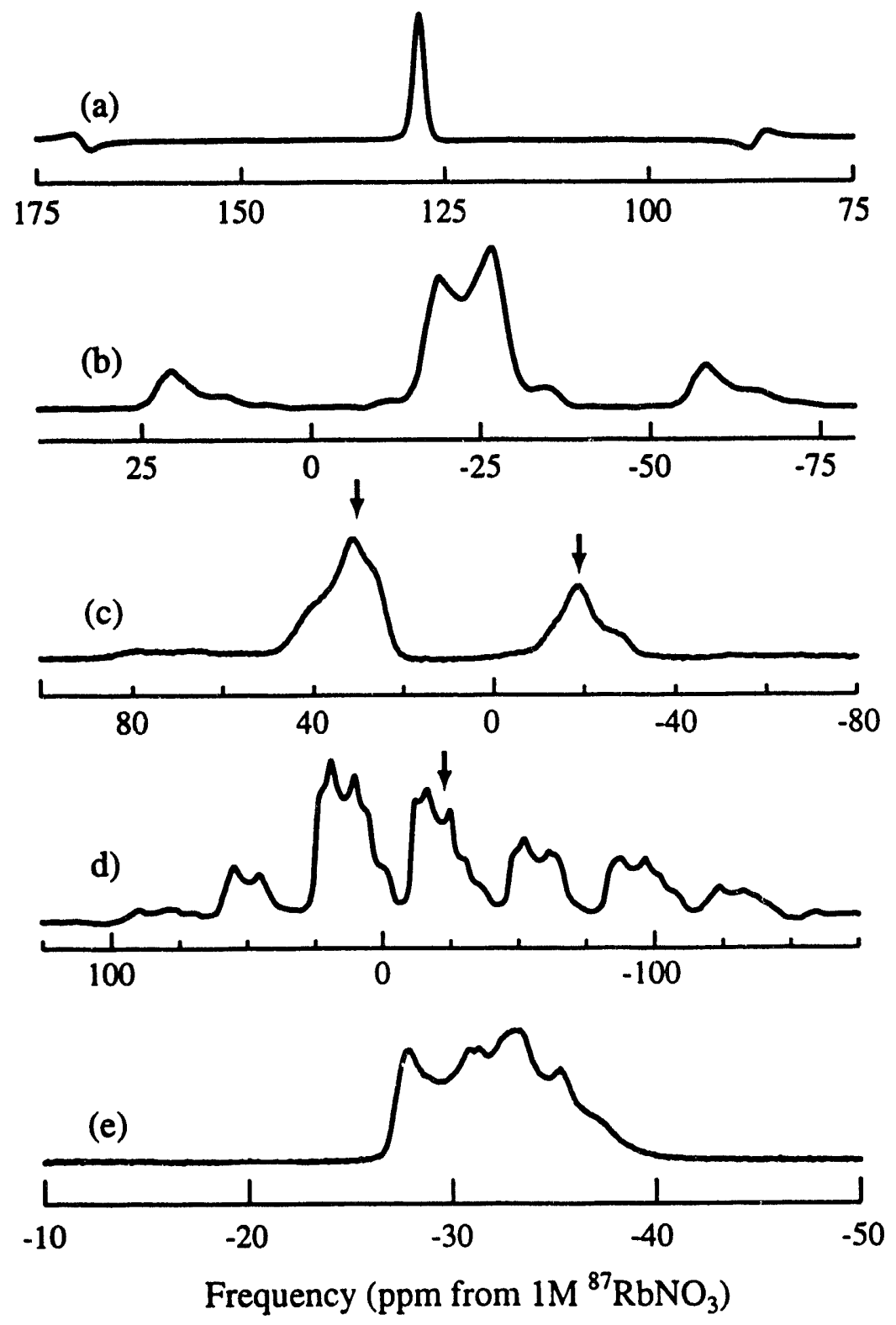

Figure $7.1{ }^{87} \mathrm{Rb}$ Salts $11.7 \mathrm{~T}$ VAS Spectra. (a) $\mathrm{RbCl}$ at $54.74^{\circ}$, (b) $\mathrm{RbClO}_{4}$ at $54.74^{\circ}$, (c) $\mathrm{Rb}_{2} \mathrm{SO}_{4}$ at $79.19^{\circ}$, (d) $\mathrm{Rb}_{2} \mathrm{CrO}_{4}$ at $54.74^{\circ}$, (e) $\mathrm{RbNO}_{3}$ at $54.74^{\circ}$.

The DAS spectra at $11.7 \mathrm{~T}$ reveal the actual isotropic shifts for each site in each compound (except the broadest site in $\mathrm{Rb}_{2} \mathrm{CrO}_{4}$ ) without the need for simulations. This has the advantage of greatly improving the accuracy of the measurement of the isotropic shifts. In the case of $\mathrm{Rb}_{2} \mathrm{SO}_{4}$, the DAS spectrum illustrates one of the classic problems with VAS spectra. 

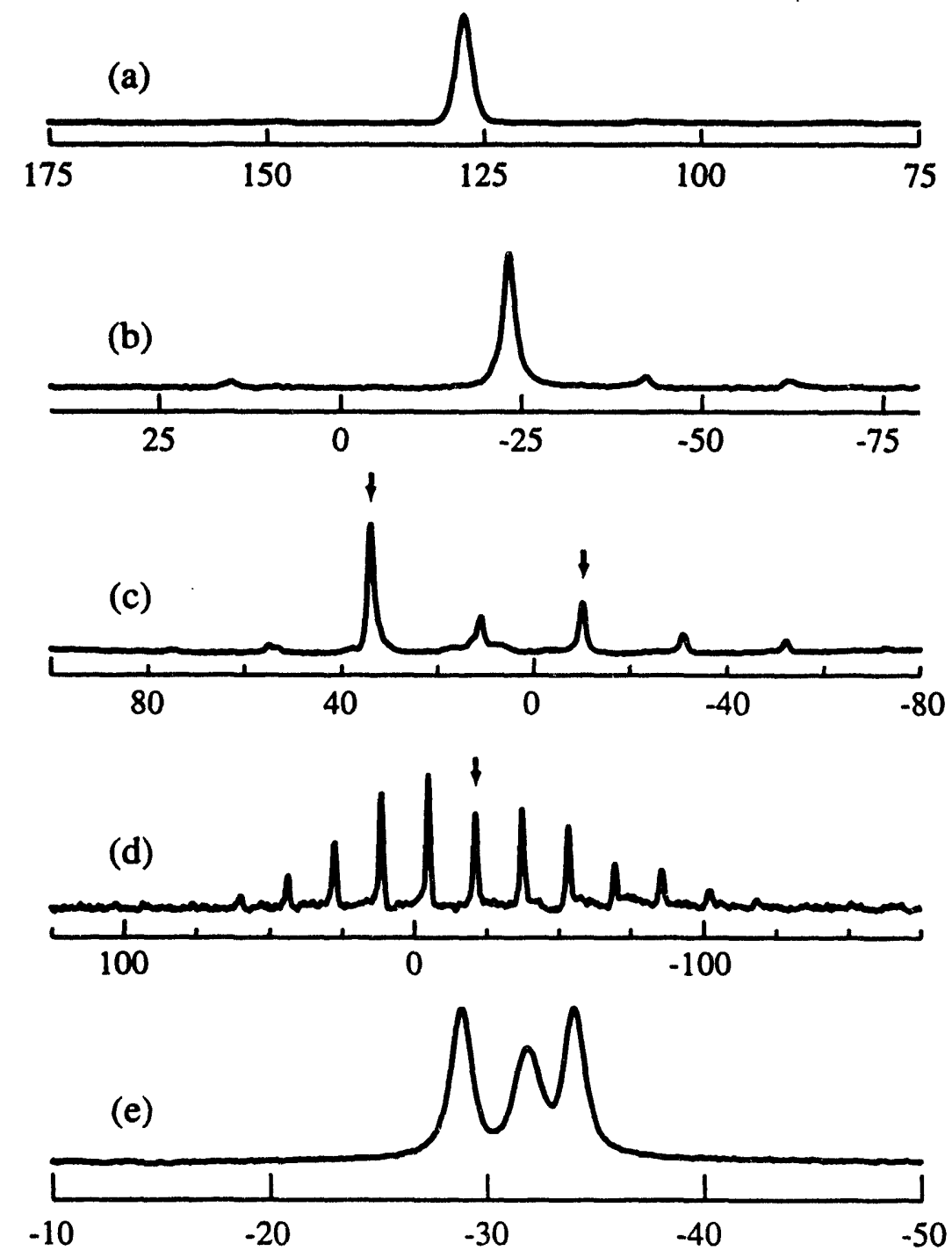

Frequency (ppm from $1 \mathrm{M}^{87} \mathrm{RbNO}_{3}$ )

Figure $7.2{ }^{87} \mathrm{Rb}$ Salts 11.7T DAS Spectra. (a) $\mathrm{RbCl}$, (b) $\mathrm{RbClO}_{4}$, (c) $\mathrm{Rb}_{2} \mathrm{SO}_{4}$, (d) $\mathrm{Rb}_{2} \mathrm{CrO}_{4}$, (e) $\mathrm{RbNO}_{3}$.

The actual isotropic shifts in this salt (as seen in the DAS spectrum, figure 7.2c) do not correspond to the highest point in the VAS spectrum (figure 7.1c), rather, the isotropic shifts in the VAS spectrum fall at the overall centers of gravity of each peak. Low intensity contributions in the wings of the VAS peaks make calculation of the center of gravity of these peaks difficult. 


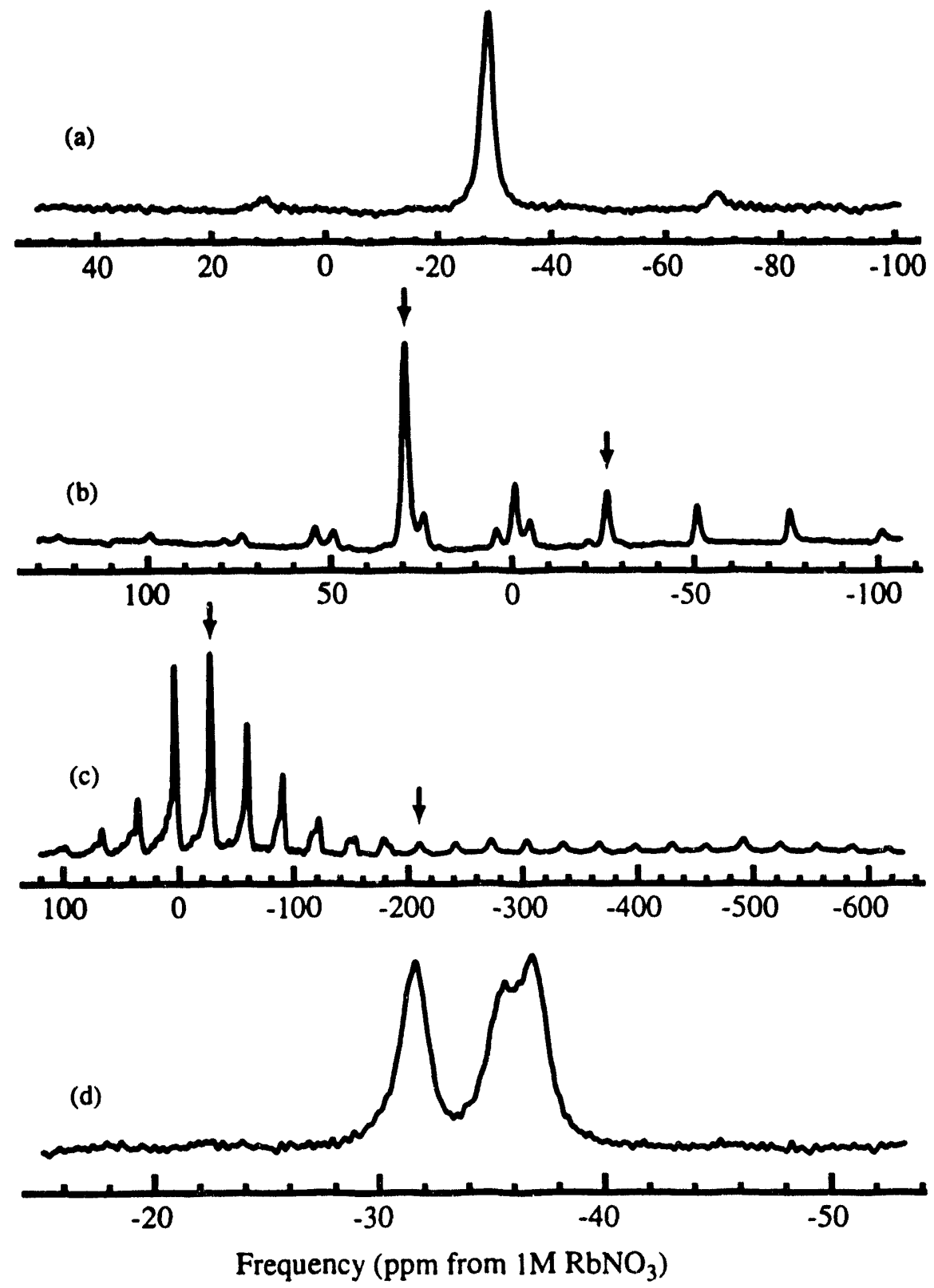

Figure $7.3{ }^{87} \mathrm{Rb}$ Salts 9.4T DAS Spectra. (a) $\mathrm{RbClO}_{4}$, (b) $\mathrm{Rb}_{2} \mathrm{SO}_{4}$, (c) $\mathrm{Rb}_{2} \mathrm{CrO}_{4}$, (d) $\mathrm{RbNO}_{3}$.

Figure 7.3 shows the DAS spectra of the same salts at a lower field strength. Notice that the spectra all have the same high resolution of the $11.7 \mathrm{~T}$ spectra in figure 7.2. However, now some of the peaks fall at different isotropic positions because of differences in the second order quadrupolar isotropic shifts (see equation 7.2 and 7.3). The cubic $\mathrm{RbCl}$ does not shift at all, since this salt has zero electric field gradients at the $\mathrm{Rb}$ nucleus (as evidenced earlier by the narrow MAS spectrum in figure 7.1a). All othei 
peaks shift slightly down field to more negative ppm values. Also, the number of spinning sidebands for a given site differs between the two fields, due to the fact that the overall size of the second-order broadening (which appears in the expressions for sideband intensities in chapter 3 ) is increased at lower field. Also, since the chemical shift scales with the field, the CSA contribution to the sidebands will actually be reduced at the lower field (this is the reason much of the ${ }^{13} \mathrm{C}$ and ${ }^{31} \mathrm{P}$ MAS NMR work is done at lower field strengths).

\begin{tabular}{llllc}
\hline Compound & $\delta_{o b s}^{9.4 T}(\mathrm{ppm})$ & $\delta_{o b s}^{11.7 T}(\mathrm{ppm})$ & $\delta_{i s o}^{(C S)}(\mathrm{ppm})$ & $P_{Q}(\mathrm{MHz})$ \\
\hline $\mathrm{RbCl}$ & $127 \pm 1$ & $127 \pm 1$ & $127 \pm 2$ & 0 \\
$\mathrm{RbClO}_{4}$ & $-28 \pm 1$ & $-23 \pm 1$ & $-14 \pm 2$ & $3.1 \pm 0.3$ \\
$\mathrm{Rb}_{2} \mathrm{SO}_{4}$ & $-25 \pm 1$ & $-10 \pm 1$ & $16 \pm 2$ & $5.3 \pm 0.2$ \\
& $29 \pm 1$ & $34 \pm 1$ & $42 \pm 2$ & $3.0 \pm 0.3$ \\
$\mathrm{Rb}_{2} \mathrm{CrO}_{4}$ & $-27 \pm 1$ & $-21 \pm 1$ & $-11 \pm 2$ & $3.3 \pm 0.3$ \\
& $-201 \pm 2$ & $\mathrm{a}$ & $\mathrm{a}$ & $\mathrm{a}$ \\
$\mathrm{RbNO}_{3}$ & $-32 \pm 1$ & $-29 \pm 1$ & $-24 \pm 2$ & $2.4 \pm 0.4$ \\
& $-36 \pm 1$ & $-32 \pm 1$ & $-25 \pm 2$ & $2.8 \pm 0.4$ \\
& $-37 \pm 1$ & $-34 \pm 1$ & $-29 \pm 2$ & $2.4 \pm 0.4$ \\
\hline
\end{tabular}

Table $7.1^{87} \mathrm{Rb}$ Isotropic Shifts and Coupling Products. The isotropic chemical shifts and

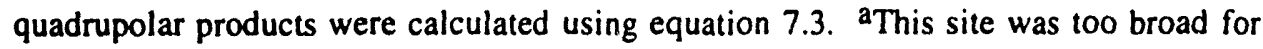
detection at 11.7T with both the DAS and MAS experiments.

In the case of $\mathrm{Rb}_{2} \mathrm{CrO}_{4}$, the second broad site at $-201 \mathrm{ppm}$ appears in the $9.4 \mathrm{~T}$ spectrum which was absent in the higher field spectrum. Also, the overall number of sidebands around the $-27 \mathrm{ppm}$ peak is greatly reduced at $9.4 \mathrm{~T}$, since the major contribution to the anisotropic broadening of this site is the chemical shift interaction. This actually may be seen in the MAS spectrum (figure 7.1d) where the individual sidebands have clearly resolved quadrupolar MAS patterns. Normally, when large numbers of sidebands result from quadrupolar coupling alone, the MAS pattern will be greatly distorted and overlap- 
ping with the sidebands. In the case of $\mathrm{Rb}_{2} \mathrm{CrO}_{4}$, this is not observed, showing that the CSA is quite large for this site. All of the measured isotropic shifts are compiled in table 7.1 below.

The two field DAS measurements were used to generate both the quadrupolar coupling products and isotropic chemical shifts for these salts using equation 7.3. These values are tabulated in table 7.1. The values of the quadrupolar products and isotropic shifts in table 7.1 may now be compared to the values arrived at by Cheng et al. ${ }^{128}$ from static simulations compiled in table 7.2. For the case of the $\mathrm{RbCl}$, our results agree exactly with those of Cheng et al. ${ }^{128}$

\begin{tabular}{lccc}
\hline Compound & $\delta_{\text {iso }}^{(C S)}(\mathrm{ppm})$ & $\eta_{Q}$ & $P_{Q}(\mathrm{MHz})$ \\
\hline $\mathrm{RbCl}$ & 128.0 & 0 & 0 \\
$\mathrm{RbClO}_{4}$ & 3.8 & 0.16 & 3.2 \\
$\mathrm{Rb}_{2} \mathrm{SO}_{4}$ & 3.0 & 0.13 & 3.2 \\
& 46.6 & 0.89 & 2.9 \\
$\mathrm{Rb}_{2} \mathrm{CrO}_{4}$ & -47.4 & 0.48 & 5.4 \\
& 52.8 & 0.75 & 12.5 \\
$\mathrm{RbNO}_{3}$ & $*$ & $*$ & $*$ \\
\hline
\end{tabular}

Table 7.2 Previously Measured ${ }^{87} \mathrm{Rb}$ Isotropic Chemical Shifts and Quadrupolar Parameters. These parameters were determined by simulating static central transition multi-site patterns with both quadrupolar and chemical shift anisotropy parameters by Cheng et al. For $\mathrm{RbNO}_{3}$, the three sites could not be resolved.

For other compounds, the agreement is much worse, indicating the difficulty of relying only on static simulations (which have a large number of parameters to adjust) in measuring quadrupolar and chemical shift parameters. The rough size of the coupling constants measured by Cheng et al. ${ }^{128}$ for the $\mathrm{RbClO}_{4}$ and $\mathrm{Rb}_{2} \mathrm{CrO}_{4}$ are in the correct range. However, in all cases except for $\mathrm{RbCl}$, the isotropic chemical shifts are quite inaccurate. Also, in the case of $\mathrm{RbNO}_{3}$, which has the strongest overlap, the static simulations fail completely. 


\section{Improvements from Multiple-Field DAS}

To improve the overall accuracy and precision for the measurement of the isotropic chemical shifts and quadrupolar coupling products, the DAS measurement should be made at more than two fields. In this case, the system of equations which relate the measured isotropic shifts to the isotropic chemical shift and second-order quadrupolar coupling products are over-determined.

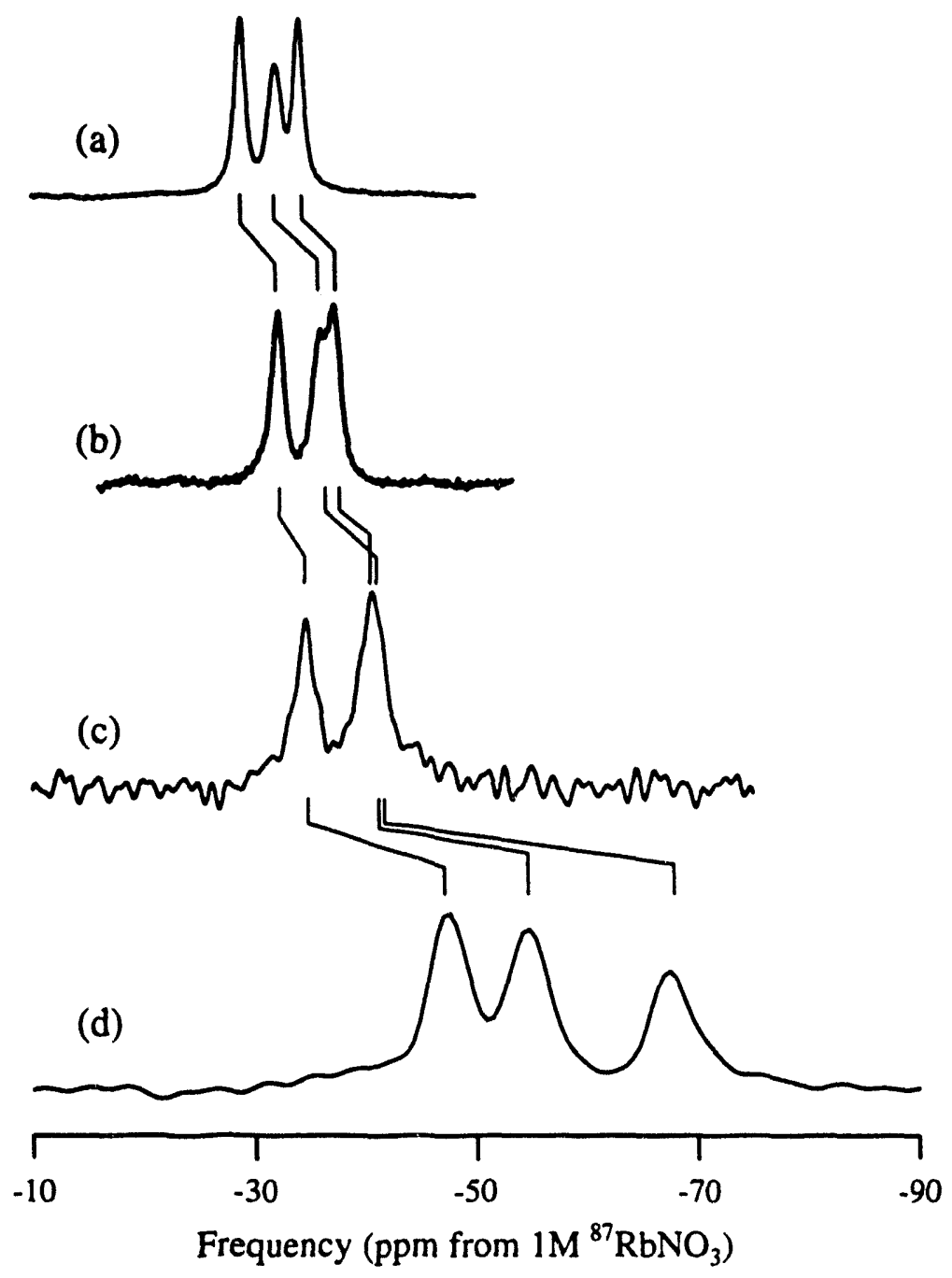

Figure 7.4 RbNO 3 Spectra at Four Field Strengths. (a) 11.7T, (b) 9.4T, (c) 7.0T and (d) 4.2T.

This opens the possibility of a linear least-squares fit of the isotropic shifts when plotted versus the reciprocal of the field strength squared. Figure 7.4 shows the $\mathrm{RbNO} 3$ spectra measured at $11.7 \mathrm{~T}(\mathrm{a}), 9.4 \mathrm{~T}(\mathrm{~b}), 7.0 \mathrm{~T}(\mathrm{c})$ and $4.2 \mathrm{~T}(\mathrm{~d})$. Notice in figure 7.4 that the 
overall resolution in ppm seems to get worse as the field strength gets larger. In fact the linewidth will remain approximately constant in units of Hertz (about $150 \mathrm{~Hz}$ in this case) and will appear larger in units of ppm (normally used in all reported measurements) as the field is reduced. This means that the error bars on the lower field isotropic shift measurements will become larger and larger. This fact must be accounted for in the linear least squares analysis of the best fits (see figure 7.5). To do this, the contribution of each point to the lease-squares chi-squared value must be weighted by the error in the measurement of that point. Figure 7.5 shows the plot of the measured isotropic shifts versus the reciprocal of the field strength squared. The best fits through each of the sets of isotropic shifts are shown.

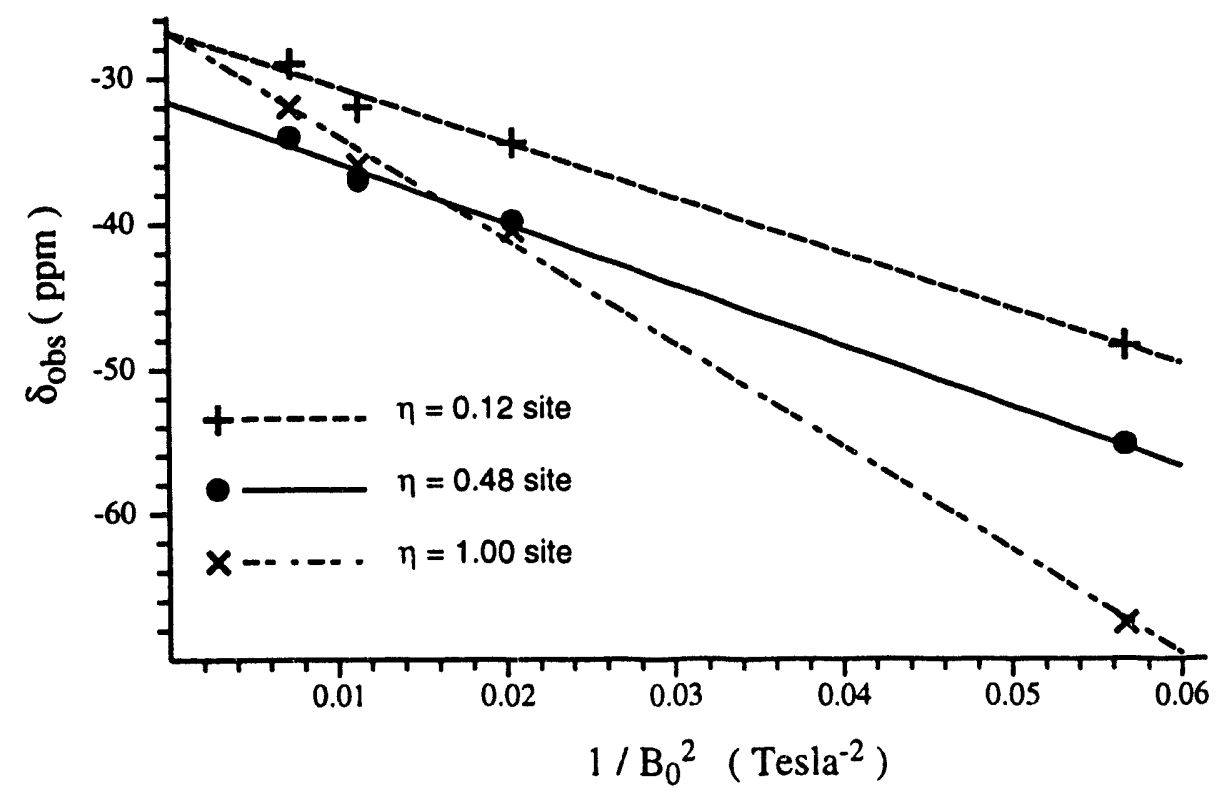

Figure 7.5 $\mathrm{RbNO}_{3}$ Linear Regression of Isotropic Shifts versus $1 / B_{0}^{2}$

This linear regression gives significant improvements in the overall errors. Figure 7.4 shows the DAS spectra at the four field strengths and Figure 7.5 shows the actual linear regression best fit. Table 7.3 gives the compiled final results and errors. As observed, the errors are about half as large as with only two fields (table 7.1). The isotropic shifts are all given in units of ppm and the quadrupolar coupling product is in units of $\mathrm{MHz}$. In addition, when these results are compared to those from single site simulations (see next 
section), the values for the quadrupolar and chemical shift parameters are much closer to the correct values.

\begin{tabular}{cccccc}
\hline$\delta_{o b s}^{4.2 T}$ & $\delta_{o b s}^{7.0 T}$ & $\delta_{o b s}^{9.4 T}$ & $\delta_{o i s}^{11.7 T}$ & $\delta_{i s o}^{(C S)}$ & $P_{Q}$ \\
\hline$-48.4 \pm 3.0$ & $-34.4 \pm 2.0$ & $-32.0 \pm 1.0$ & $-29.0 \pm 1.0$ & $-26.8 \pm 0.8$ & $1.72 \pm 0.06$ \\
$-67.5 \pm 3.0$ & $-39.8 \pm 2.0$ & $-36.0 \pm 1.0$ & $-32.0 \pm 1.0$ & $-26.8 \pm 0.8$ & $2.36 \pm 0.04$ \\
$-55.3 \pm 3.0$ & $-40.2 \pm 2.0$ & $-37.0 \pm 1.0$ & $-34.0 \pm 1.0$ & $-31.6 \pm 0.8$ & $1.81 \pm 0.05$ \\
\hline
\end{tabular}

Table $7.3{ }^{87} \mathrm{RbNO}_{3}$ Multiple Field DAS Results. Isotropic chemical shifts and quadrupolar products were calculated from a linear regression analysis of the isotropic shifts versus $1 / B_{0}^{2}$ as in figure 7.6 .

\section{Improvements from MAS-Detected DAS}

MAS detected DAS ${ }^{50}$ was performed at both $9.4 \mathrm{~T}$ and $11.7 \mathrm{~T}$ (figures 7.6 and 7.7 respectively.) These spectra show a high resolution DAS dimension as well as a purephase MAS detected anisotropic dimension. Slices through each DAS peak yield accurate MAS lineshapes for each site. Figure 7.8 shows the simulation of each of the three sites and table 7.4 gives the most precise (because there is only one external standard) and accurate (essentially more data points are effectively involved in the calculation than in multiple field methods) ${ }^{87} \mathrm{RbNO}_{3}$ quadrupolar coupling and chemical shift parameters measured. In addition, by using both fields, even greater accuracy may be achieved by simulating both field spectra at the same time. This approach has been used previously for multiple site ${ }^{17} \mathrm{O}$ spectra ${ }^{48}$ (see chapter 8 ). The pulse sequence used to collect these spectra is the double-hop DAS sequence described by Mueller et al. ${ }^{50}$ This sequence achieves pure-absorption mode spectra by taking a hypercomplex data set in $t_{1}$. The overall signal-to-noise ratio is greatly reduced as compared to the hypercomplex SEDAS experiment (by a factor of 8 ) due to the second $z$-filter storage period (used to store magnetization while we reorient the spinner between the second DAS angle to the magic-angle $54.74^{\circ}$ ) and the lack of an echo in the second dimension. However, in the case of 
${ }^{87} \mathrm{RbNO}_{3}$, the relaxation times and absolute signal intensity make collection of a MAS detected DAS spectrum quite feasible.
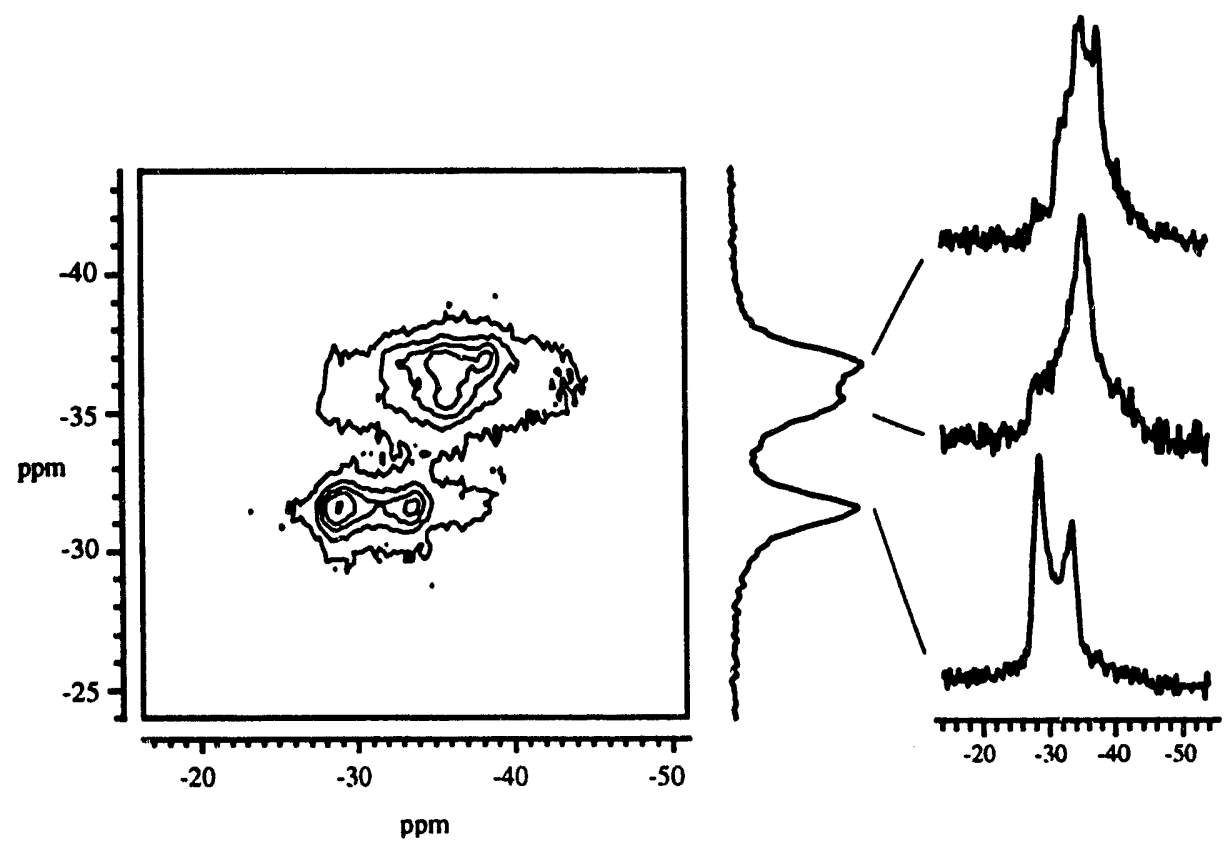

Figure 7.6 $\mathrm{RbNO}_{3}$ 9.4T 2D MAS detected DAS Contour Plot. Single site MAS slices through each isotropic peak in the DAS dimension have been extracted and are displayed to the right of the contour plot.

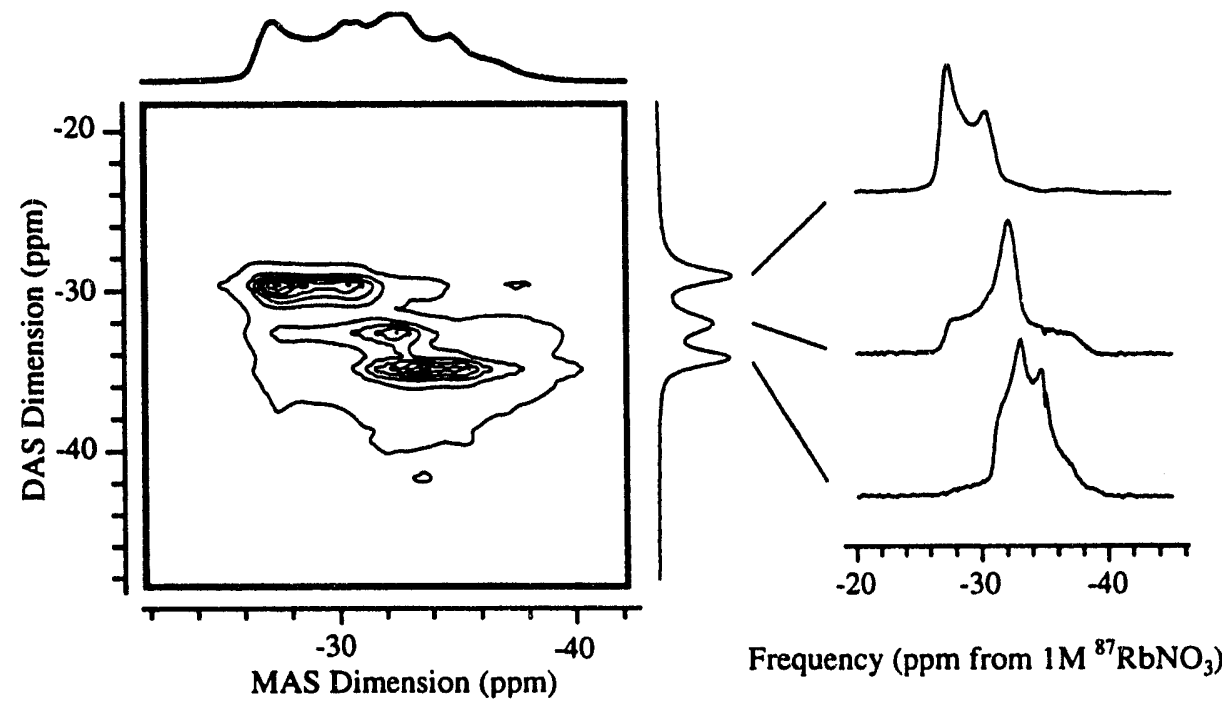

Figure 7.7 $\mathrm{RbNO}_{3}$ 11.7T 2D MAS detected DAS Contour Plot. Single site MAS slices through each isotropic peak in the DAS dimension have been pulled out and are displayed to the right of the contour plot.

In the 9.4T MAS detected DAS spectrum, figure 7.6, the site with a nearly zero asymmetry parameter at $-32 \mathrm{ppm}$ is clearly separated from the other two sites. The slice through 
this peak may be fit easily with a single high speed MAS pattern. The other two sites overlap too much and the sum of the slices through these peaks must be simulated with two patterns. In the case of 11.7T MAS detected DAS, figure 7.7, all three sites are cleanly separated and may be simulated individually. Figure 7.8 shows each of the three slices through the isotropic DAS peaks at $11.7 \mathrm{~T}$, along with the best fit simulations. The parameters and error bars for these simulations are given in table 7.4 below.

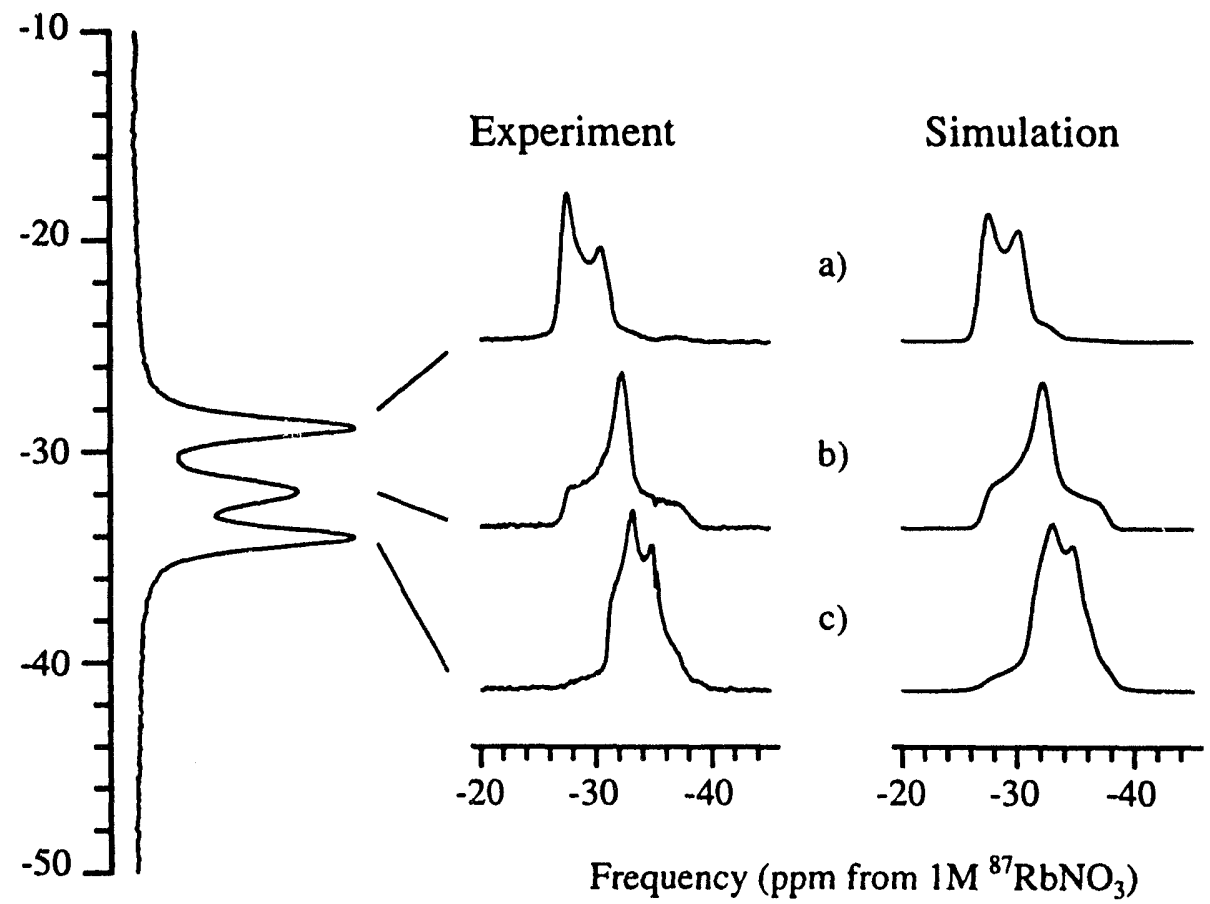

Figure 7.8 $\mathrm{RbNO}_{3}$ Single Site MAS Slices and Simulations at 11.7T. Best fit parameters are given in table 7.3.

The isotropic shifts from these simulations agree quite well with the results from the four field linear regression fit of the isotropic shifts. The same type of simulation was performed on the ${ }^{87} \mathrm{RbClO}_{4} \mathrm{MAS}$ spectrum, yielding the results in table 7.4. The advantage with the MAS detected DAS method is that in this case, only a single field strength is needed with only a single experiment. The errors from this method are even less for the quadrupolar coupling constants than in the multiple field experiments of the previous section. The errors in the measurement of the isotropic chemical shifts, however, are larger since these are primarily systematic errors due to the external $1 \mathrm{M}^{87} \mathrm{RbNO}_{3}$ fre- 
quency reference. The combination of both the multiple field measurements and the MAS detected DAS experiments leads to the highest overall accuracy and precision in the determination of these parameters.

\begin{tabular}{llll}
\hline Compound & $\delta_{i s 0}^{(C S)}(\mathrm{ppm})$ & $C_{Q}(\mathrm{MHz})$ & $\eta$ \\
\hline $\mathrm{RbClO}_{4}$ & $-16.2 \pm 1.0$ & $3.20 \pm 0.05$ & $0.10 \pm 0.05$ \\
$\mathrm{RbNO}_{3}$ & $-26.2 \pm 1.0$ & $1.83 \pm 0.05$ & $0.12 \pm 0.05$ \\
& $-26.8 \pm 1.0$ & $2.39 \pm 0.07$ & $1.00 \pm 0.05$ \\
& $-30.9 \pm 1.0$ & $1.91 \pm 0.05$ & $0.48 \pm 0.05$ \\
\hline
\end{tabular}

Table $7.4{ }^{87} \mathrm{Rb}$ Isotropic Shifts from MAS Simulations. The $\mathrm{RbClO}_{4}$ values come from the simulation of the MAS spectrum (figure 7.2b) while the $\mathrm{RbNO}_{3}$ values come from the simultaneous simulation of the 9.4T and 11.7T slices from figures 7.7 and 7.8 .

Finally, the quadrupolar coupling constants for ${ }^{87} \mathrm{RbNO}_{3}$ may be compared to those measured by Segel ${ }^{136}$. In those low field measurements, he measured coupling constants of $1.76,1.80$ and $2.20 \mathrm{MHz}$ and asymmetry parameters of $0.17,0.48$ and 0.91 for the three sites respectively. These are in very good agreement with the values measured with DAS experiments.

\section{Theory of Coupling Constants from Crystal Structure}

The $\mathrm{RbNO}_{3}$ coupling constants also provide a good example to demonstrate how to use the measured quadrupolar information to assign resonances to actual sites in the crystal structure. To do this, we assign a point charge to each of the atoms in the $\mathrm{RbNO}_{3}$ crystal structure. If for instance, we choose +1 for the rubidium atoms and $-1 / 3$ for each of the oxygen atoms, we inay then calculate EFG tensor at each rubidium site. To do this, we use the unit cell centered at the origin and the 26 unit cells which directly surround the origin. The electric field gradients are calculated using the formula given below. 


$$
\begin{aligned}
& V\left(x_{0}, y_{0}, z_{0}\right)=\frac{-e q}{r}=\frac{-e q}{\sqrt{\left(x-x_{0}\right)^{2}+\left(y-y_{0}\right)^{2}+\left(z-z_{0}\right)^{2}}} \\
& \frac{\partial^{2} V\left(x_{0}, y_{0}, z_{0}\right)}{\partial \alpha \partial \beta}=V_{\alpha \beta}=\frac{e q}{r^{5}}\left(r^{2} \delta(\alpha-\beta)-3 \alpha \beta\right)
\end{aligned}
$$

Where $\alpha, \beta$ are coordinates $x, y, z$ and $\delta(\alpha-\beta)$ is a Dirac delta function. With each electric field gradient (EFG) tensor element known, we may then diagonalize the tensor to get the principal axis values for $V_{x x}, V_{y y}$ and $V_{z z}$. To convert these values into $C_{Q}$ and $\eta$ values, we need to use the relationships that relate $V_{\alpha \beta}$ to quadrupolar coupling constants. In addition we need to know the Sternheimer anti-shielding factor. In the case of $\mathrm{RbNO}_{3}$ we have calculated EFG values (see table 7.5) for a variety of point charge distributions. Changing the values of the charges changes the absolute size of the EFG tensor values, but does not appreciably change the asymmetry parameters. Therefore, we may assign each DAS peak to a site in the $\mathrm{RbNO}_{3}$ crystal structure (unit cell not shown).

\begin{tabular}{|llllll|}
\hline Rb Charge & N Charge & O Charge & Site & $C_{Q}$ (a.u.) & $\eta$ \\
\hline \hline+0.70 & -0.10 & -0.20 & 1 & 3.5 & 0.31 \\
& & & 2 & 3.4 & 0.59 \\
& & & 3 & 3.5 & 0.97 \\
\hline+1.00 & -0.10 & -0.30 & 1 & 3.4 & 0.30 \\
& & & 2 & 3.4 & 0.61 \\
& & & 3 & 3.5 & 0.94 \\
\hline+0.60 & +0.10 & -0.23 & 1 & 3.4 & 0.29 \\
& & & 2 & 3.4 & 0.72 \\
& & & 3 & 3.5 & 0.81 \\
\hline+0.40 & -0.10 & -0.10 & 1 & 3.4 & 0.32 \\
& & & 2 & 3.4 & 0.55 \\
& & & 3 & 3.5 & 0.97 \\
\hline
\end{tabular}

Table 7.5 ${ }^{87} \mathrm{RbNO}_{3}$ EFG Values From Crystal Structure. These calculations were carried out over a large number of unit cells and the EFG values were calculated and averaged for the thirty inner most $\mathrm{Rb}$ sites (out of almost 250 total).

\section{Application of CPDAS to organic compounds}


The use of solid state NMR to study biologically active and interesting compounds has been one of the longtime goals of many researchers. Techniques such as rotational resonance $\left(\mathrm{R}^{2}\right)$, spin echo double resonance (SEDOR), rotational echo double resonance (REDOR) and transferred echo double resonance (TEDOR) have been used successfully to measure distances at specific sites in a number of biological samples by research groups at MIT and Washington University. ${ }^{137,138}$ These techniques look primarily at the ${ }^{1} \mathrm{H},{ }^{19} \mathrm{~F},{ }^{13} \mathrm{C}$ and ${ }^{15} \mathrm{~N}$ nuclei in labeled compounds. The important oxygen nucleus has been studied much less. The primary reasons for this lack of $17 \mathrm{O}$ information stems from its low gyromagnetic ratio $\left(1 / 7\right.$ of $\left.{ }^{1} \mathrm{H}\right)$, strong quadrupolar interactions and low natural abundance $(0.037 \%)$. Isotopic substitution may be used to over come the last problem and large magnetic fields may be used to fight the first (and to some degree the second). The strong second-order quadrupolar broadening in ${ }^{17} \mathrm{O}$ compounds is the largest obstacle remaining.

Recent developments in DAS have allowed us to begin to look more closely at ${ }^{17} \mathrm{O}$. Specifically in the case of biological samples, decoupling of the ${ }^{1} \mathrm{H}$ nuclei is essential for high resolution. In addition, the long ${ }^{17} \mathrm{O}$ relaxation times and low sensitivity may be overcome with cross polarization techniques (as described in chapter 5). We have begun preliminary studies of L-alanine, one of the simplest amino acids which is present in virtually all proteins and peptides. The $20 \%$ enriched sample was made by $\mathrm{H}$. Zimmerman by acid catalyzed exchange of oxygen in ${ }^{17} \mathrm{O}$ labeled water. The relaxation times in this compound are quite favorable for DAS, with a $700 \mathrm{~ms}^{1} \mathrm{H}$ relaxation time (this determines the experimental repetition rate) and $2.5 \mathrm{~s}$ for the ${ }^{17} \mathrm{O}$ (which determines the minimum rotor reorientation time).

For the cross polarization experiments on ${ }^{17} \mathrm{O}$ labeled $\mathrm{L}$-alanine at $7.04 \mathrm{~T}$ the $3 / 4$ " static coil DAS probe desigred by Mueller et al. ${ }^{51}$ was refitted with a double tuned ${ }^{1} \mathrm{H}$ ${ }^{17} \mathrm{O}$ rf circuit capable of absorbing $500 \mathrm{~W}$ decoupling pulses on the ${ }^{1} \mathrm{H}$ channel $(301.2$ 
MHz). The circuit used is a standard one described previously by Doty et al. ${ }^{139,140}$ The ${ }^{1} \mathrm{H}$ and ${ }^{17} \mathrm{O}$ central transition selective pulses were both approximately $7 \mu \mathrm{s}$. The dwell times were $12.5 \mu \mathrm{s}$ in the $t_{2}$ dimension and $18 \mu \mathrm{s}$ in the $t_{1}$ dimension (after shearing). The angle pair was the usual $0^{\circ}-63.43^{\circ}(k=5)$ to obtain maximum CP efficiency.

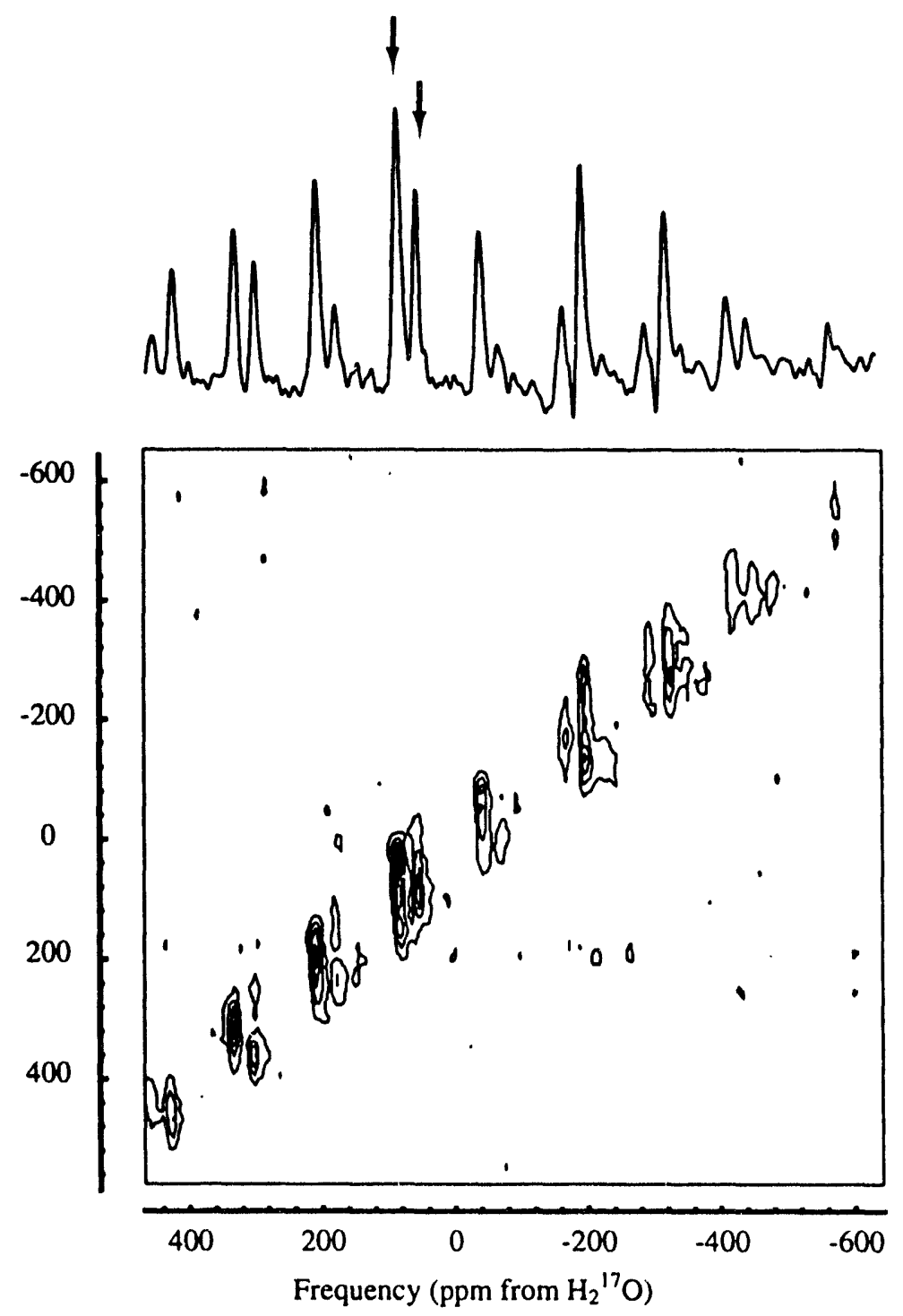

Figure $7.9{ }^{1} \mathrm{H}$ Decoupled Two-Dimensional ${ }^{17} \mathrm{O}$ CPDAS Spectrum of Alanine at 7.0T. The experimental parameters are given in the text. The two isotropic peaks are labeled with arrows. All other peaks in the DAS dimension are spinnirig sidebands.

The spinning rate was $6 \mathrm{kHz}$ and the hopping time was $35 \mathrm{~ms}$. In these experiments, 256 points were taken in the anisotropic dimension and 117 in the isotropic DAS dimension. The data in figure 7.9 was zero-filled to $256 \times 256$ for final processing. L-Alanine has 
two crystallographically distinct oxygen sites in the unit cell ${ }^{141}$. The two isotropic peaks for the distinct oxygen sites in L-alanine were observed at $51 \pm 4$ and $80 \pm 4 \mathrm{ppm}$ from the ${ }^{17} \mathrm{O}$ labeled water standard. All other peaks in the spectrum in figure 7.9 are spinning sidebands.

The spectra of alanine taken at $11.7 \mathrm{~T}$ used a standard single tuned probe. ${ }^{51}$ At this high (500 MHz) proton frequency, no decoupling could be achieved and the oxygen lines are significantly broader (almost a factor of 10 ) than in the decoupled spectrum at 7.04T.

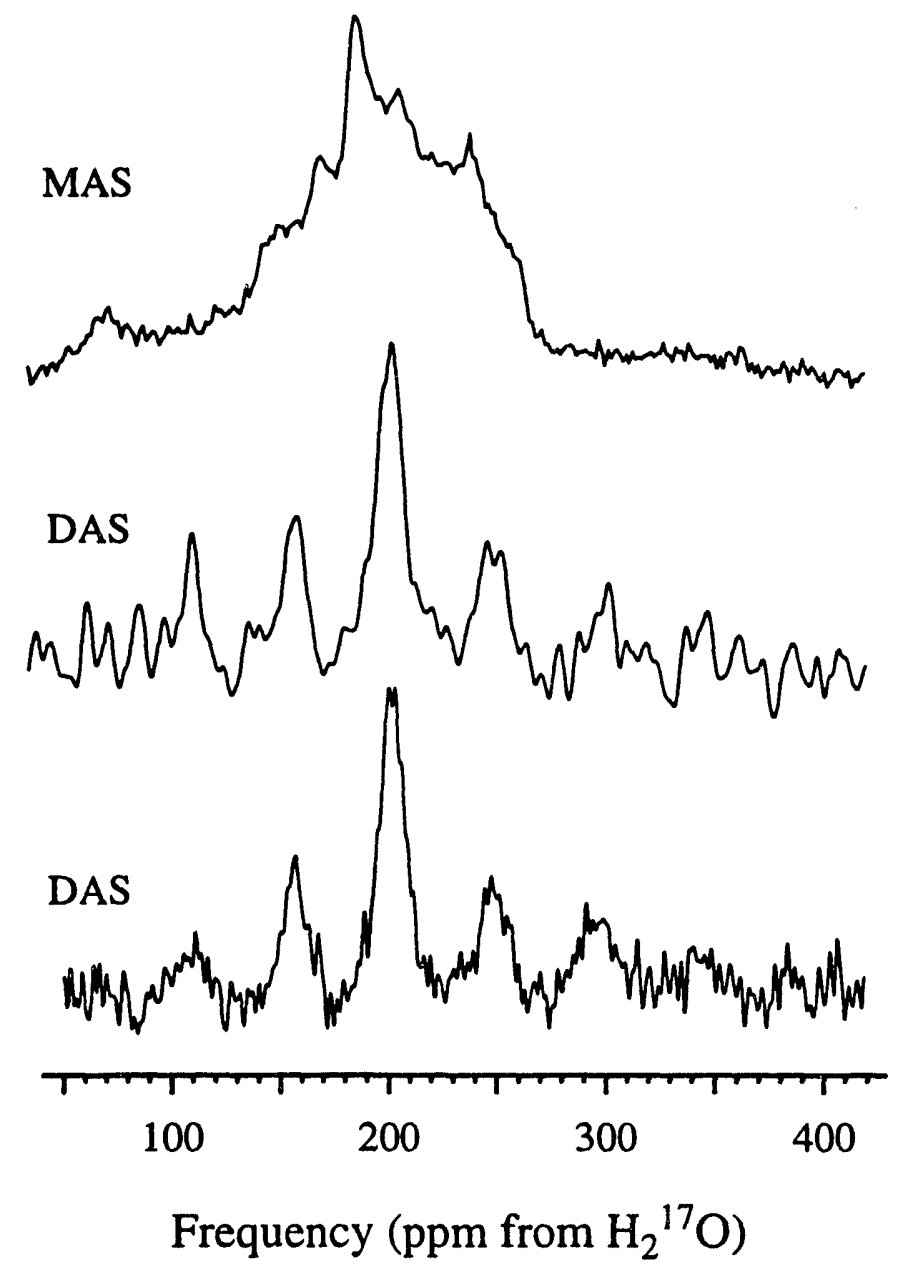

Figure 7.10 Undecoupled ${ }^{17} \mathrm{O}$ MAS and DAS Spectra of Alanine at 11.7T. The experimental parameters for these experiments are given in the text. The isotropic peak in the DAS spectrum occurs at $200 \mathrm{ppm}$; all other peaks are spinning sidebands. 
The $\pi / 2$ selective pulses were again $7.0 \mu \mathrm{s}$; most other parameters were similar to those used at $7.04 \mathrm{~T}$. Figure 7.10 shows both the MAS and two DAS spectra for the same alanine sample as in figure 7.9. The MAS spectrum shows a broad powder pattern with a number of singularities. The two peaks in the DAS spectrum are not clearly resolved and are both assigned an isotropic shift of $200 \pm 7 \mathrm{ppm}$. Using the two field results (just as in the case of the ${ }^{87} \mathrm{Rb}$ salts of the previous section) we may calculate the isotropic chemical shifts and quadrupolar coupling products for the two sites in alanine. These results are compiled in table 7.6 below.

\begin{tabular}{|ccccc|}
\hline Site & $\delta_{o b s}^{7.04 T}$ & $\delta_{o b s}^{11.7 T}$ & $P_{Q}(\mathrm{MHz})$ & $\delta_{\text {iso,cs }}$ \\
\hline 1 & $51 \pm 4 \mathrm{ppm}$ & $200 \pm 7 \mathrm{ppm}$ & $8.1 \pm 0.3 \mathrm{MHz}$ & $285 \pm 8 \mathrm{ppm}$ \\
2 & $80 \pm 4$ & $200 \pm 7$ & $7.2 \pm 0.3$ & $268 \pm 8$ \\
\hline
\end{tabular}

Table 7.6 ${ }^{17} \mathrm{O}$ L-Alanine DAS Results. Multiple field measurements from figures 7.9 and 7.10 are tabulated along with the calculated quadrupolar products and isotropic chemical shifts. The error bars are indicated and arise from the overall width of the peaks in the DAS spectra.

These values for the quadrupolar coupling products are in good agreement with the size of the quadrupolar coupling constant measured for the carboxyl oxygen atoms in similar compounds with NQR. Additional experiments are currently underway which will apply the techniques of CPDAS and DAS to other organic compounds with the long term goal of examining larger biologically active molecules. 


\section{Chapter 8}

\section{Application of DAS to silicate materials}

The use of dynamic-angle spinning to study silicate samples has so far proven to be probably the most important application of this experiment. The ${ }^{17} \mathrm{O}$ nucleus, along with the ${ }^{29} \mathrm{Si}$ nucleus, is in one of the pivotal locations in silicate materials. There are two common types of oxygen environments in silicate samples, first those which are covalently bonded between two other atoms (bridging oxygen atoms), usually silicon, (but also others, for example aluminum, phosphorus or boron) and second those which have a single covalent bond to a silicon atom and therefor a net negative charge balanced by neighboring cations such as sodium, potassium or calcium. A third type has been found in some high pressure systems, where oxygen forms three covalent bonds to neighboring silicon atoms. This third type is very uncommon and will not be discussed here. The use of NMR to look at ${ }^{17} \mathrm{O}$ has becoming increasingly popular in the last ten years, due to the construction of high field magnets (greater than $9 \mathrm{~T}$ ) and high speed spinning probes (greater than $10 \mathrm{kHz}$ ). The measurement of $17 \mathrm{O}$ quadrupolar coupling parameters has been an important goal, since these parameters are strongly correlated to the local microscopic structures ${ }^{142}$. Some of the most important early contributions were made by Oldfield et al. ${ }^{72,73,78,97,104,143-151}$ Included in this list of references are pioneering MAS experiments on a series of modified silicate materials, including wollastonite, diopside and forsterite. In recent years, DAS has been applied to the study of similar materials and the reader is referred to work done by Pines et al. ${ }^{45-48}$ Some of this work will be discussed in this thesis, as well as the previously mentioned thesis by Mueller. ${ }^{15}$

\section{Crystalline Silicates}

In this section, I will describe studies of a variety of ${ }^{17} \mathrm{O}$ labeled pyroxene mineral samples which have previously been examined by Timken et al. ${ }^{150}$ with MAS and by 
Mueller et al..$^{45}$ using DOR and DAS. Specifically, the isotropic shifts and quadrupolar coupling products (defined below) of diopside, clinoenstatite, forsterite, wollastonite and larnite were measured ${ }^{48}$ In the past, in order to measure these parameters using NMR, the static, magic angle spinning (MAS) and variable angle spinning (VAS) spectra for compounds containing half-odd integer nuclear spins had been deconvoluted into individual lines using powder pattern simulation programs (see chapter 2). This type of analysis is often quite accurate. However, when more than two or more overlapping sites are present, it is difficult to extract meaningful parameters without significant errors. As has been shown throughout this thesis, the application of dynamic angle spinning (DAS) NMR to systems with quadrupolar nuclei allows significant narrowing of the MAS linewidths, usually by more than an order of magnitude and therefore a corresponding increase in accuracy.

For nuclei with large quadrupolar coupling constants and small dipolar couplings due to either low gyromagnetic ratios or low natural abundance (less than 50\%), DAS may often give substantial narrowing of the lines without generating the large number of spinning sidebands often found in double rotation experiments (DOR). This is especially important when there are a large number of magnetically inequivalent sites, such as in wollastonite which has nine distinct oxygen sites. As has been mentioned earlier in this thesis, by comparing the DAS spectra collected at two different magnetic field strengths the isotropic peaks are seen to shift (in ppm), just as in the studies of ${ }^{87} \mathrm{Rb}$ salts (see chapter 7). This is expected as the isotropic peak in a DAS experiment expressed in units of parts per million (ppm) is actually the sum of the isotropic chemical shift and the isotropic 2nd-order quadrupolar shift (see equation 7.1 and discussion).

$$
\delta_{o b s}=\delta_{i s o, c s}+\delta_{i s o}^{(2 Q)}
$$

The observed isotropic chemical shift (in ppm) is constant at all field strengths and therefore doesn't exhibit any shift between $9.4 \mathrm{~T}$ and $11.7 \mathrm{~T}$. However, the isotropic $2 \mathrm{nd}$ or- 
der quadrupolar shift (in ppm) is inversely proportional to the square of field strength and therefore becomes much smaller as field strength is increased.

$$
\begin{aligned}
\delta_{i s o}^{(2 Q)} & =\frac{\omega_{i s o}^{(2 Q)}}{\omega_{l}} \times 10^{6} \\
& =\frac{-3 \times 10^{6} C_{Q}^{2}\left(I(I+1)-\frac{3}{4}\right)}{40 \omega_{l}^{2} I^{2}(2 I-1)^{2}}\left(1+\frac{\eta_{Q}^{2}}{3}\right) \\
& =\frac{-3 \times 10^{6}\left(I(I+1)-\frac{3}{4}\right)}{40 \omega_{l}^{2} I^{2}(2 I-1)^{2}} P_{Q}^{2} \\
P_{Q} & \equiv C_{Q}\left(1+\frac{\eta_{Q}^{2}}{3}\right)^{\frac{1}{2}}
\end{aligned}
$$

Substituting in the values for ${ }^{17} \mathrm{O}\left(I=5 / 2, \omega_{l, 9.4 T}=54.245 \mathrm{MHz}, \omega_{l, 11.7 T}=67.898 \mathrm{MHz}\right)$ yields the following pair of linear equations for $\delta_{i s o, c s}$ and $P_{Q}$ where these are given in units of ppm and $\mathrm{MHz}$ respectively.

$$
\begin{aligned}
& \delta_{o b s}^{9.4 T}=\delta_{i s o, c s}-2.03691 P_{Q}^{2} \\
& \delta_{o b s}^{11.7 T}=\delta_{i s o, c s}-1.30476 P_{Q}^{2}
\end{aligned}
$$

In fact using the isotropic shifts from two fields allows one to solve two simultaneous equations relating the coupling product $P_{Q}$ and the isotropic chemical shift $\delta_{\text {iso,cs }}$.

All of the ${ }^{17} \mathrm{O}$ labeled crystalline mineral samples were prepared by Prof. J. Stebbins and coworkers following a procedure already reported ${ }^{14,45}$. The diopside was isotopically enriched with ${ }^{17} \mathrm{O}$ uniformly to the $20 \%$ level, while all other samples were $40 \%$ enriched. The phase identities and stoichiometry of these materials were all analyzed using ${ }^{29} \mathrm{Si}$ and ${ }^{17} \mathrm{O}$ NMR and had shifts which agreed with previously reported values. In addition, these findings were confirmed by powder $x$-ray diffraction. The forsterite sample was slightly off-stoichiometry and contained $25 \%$ clinoenstatite but this did not affect the final NMR measurements significantly. The unit cell structures of these compounds has been reported before as well ${ }^{152-156}$ and are shown below in figure 8.1. 

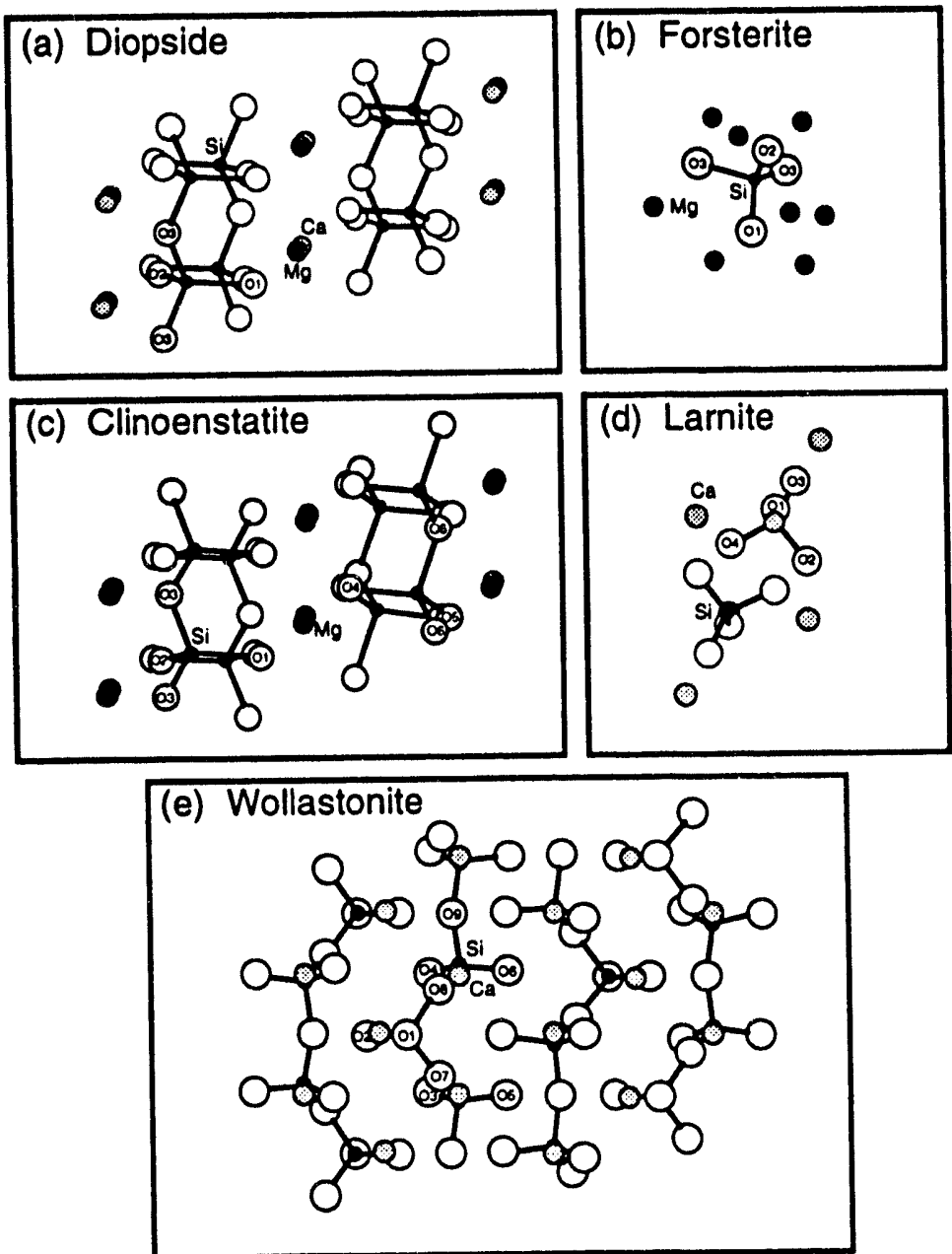

Figure 8.1 Crystal Structures of Some Pyroxene Silicate Minerals. The unit cells for diopside, clinoenstatite, wollastonite, larnite and forsterite are shown. The numbers indicate the distinct sites for each type of atom in the crystal structure. This figure is taken with permission from the thesis by Mueller ${ }^{15}$.

All of the NMR spectra at $9.4 \mathrm{~T}$ were recorded on a Bruker AM-400 spectrometer in a $89 \mathrm{~mm}$ widebore magnet, while the spectra at $11.7 \mathrm{~T}$ were recorded on a Chemagnetics CMX-500 spectrometer in a $89 \mathrm{~mm}$ widebore magnet. The DAS probes used for these experiments were homebuilt using a design detailed by Mueller, et al..$^{15,51}$ The pulse sequences and phase cycles used to collect the data were the original DAS type described previously in chapter 3 . The rf-pulse widths were calibrated to selectively excite only the central $(-1 / 2$ to $1 / 2)$ transition of $17 \mathrm{O}$ and were usually in the range of 4 to 6 $\mu$ s for the $\pi / 2$ pulses (equivalent to a $\pi / 6$ solution pulse). All data was taken with $k=1$ 
where the first angle was set at $\theta_{1}=37.38^{\circ}$ and the second angle set at $\theta_{2}=79.19^{\circ}$. The usual hopping time between these two angles ranged from $25 \mathrm{~ms}$ to $35 \mathrm{~ms}$, which was significantly shorter than the $T_{1}$ for these compounds. A $1 \mathrm{~s}$ to $5 \mathrm{~s}$ recycle delay was used for most of the experiments and the spinning rate was usually between 6 and $7 \mathrm{kHz}$. We sampled between 128 and $512 t_{1}$ points for these compounds giving a digital resolution of approximately $0.5 \mathrm{ppm}$. All ${ }^{17} \mathrm{O}$ peaks were referenced externally to a sample of $37 \%$ $\mathrm{H}_{2}{ }^{17} \mathrm{O}$ in a small ampoule placed inside a DAS rotor.

All of the simulations of quadrupolar powder patterns were done on a Stardent Titan computer. To extract the quadrupolar parameters, a program, MINUITQ, was written which calculates 2 nd order quadrupolar powder patterns spinning about any single axis (see appendix to this thesis). This program simulates only the central transition for half-odd integer nuclei and assumes that no intensity is lost in spinning sidebands. The experimental spectra were fit using a standard AMOEBA simplex routine from Numerical Recipes in FORTRAN ${ }^{157}$ or MINUIT, a minimization package from CERN. These algorithms allow rapid convergence by minimizing the root mean square deviation between the simulated and the experimental spectra. Each fit takes approximately 2,000 to 10,000 iterations to achieve a best fit with each iteration taking about 0.4 seconds per powder pattern. By using the quadrupolar parameters and isotropic chemical shift values determined directly from the one-dimensional DAS spectra, we are able to fix the isotropic shift and the value of $P_{Q}$ which limits the simplex to only 4 variable parameters per site, which are the asymmetry parameter, $\eta_{Q}$, the total intensity, the lorentzian broadening, and the Gaussian broadening for each site, under rapid magicangle spinning. Finally, we are able to fit multiple experimental spectra simultaneously. Thus by simulating MAS spectra at both $9.4 \mathrm{~T}$ and $11.7 \mathrm{~T}$, we may place a large rumber of constraints on our simulations. This allows more exact determination of the asymmetry parameter, $\eta_{Q}$, and therefore the quadrupolar coupling constant, $C_{Q}$. 
Figure 8.2 shows the DAS, DOR and MAS spectra of these six minerals previously reported. ${ }^{15,45}$ It is observed that in all cases, the DAS spectra show the same number of isotropic sites as are present in the crystal structure. In the wollastonite spectrum, two of the bridging sites overlap at $28 \mathrm{ppm}$, giving a peak twice as intense as the third bridging site at $22 \mathrm{ppm}$.

\section{Magic-Angle Spinning Dynamic-Angle Spinning Double Rotation}
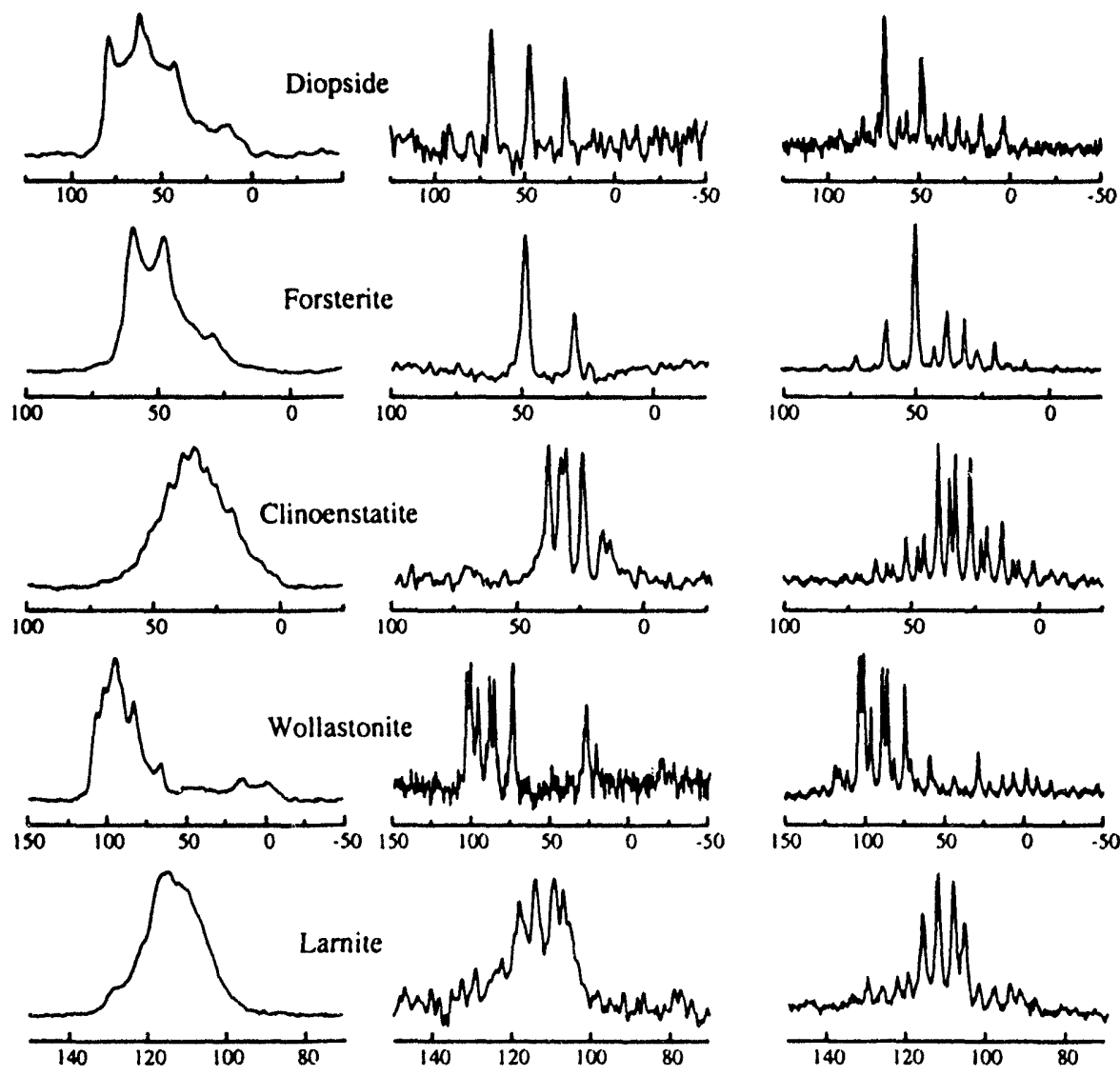

Frequency (ppm from $\mathrm{H}_{2}{ }^{17} \mathrm{O}$ )

Figure 8.2 Crystalline 9.4T DAS, DOR and MAS Spectra. The MAS spectra are very similar to those observed by Timken et al. ${ }^{150}$ and all spectra are shown with permission from the thesis by Mueller. ${ }^{15}$ The MAS and DOR spectra were taken with standard onepulse experiments while for the DAS spectra the original DAS pulse sequence was used.

The large number of spinning sidebands in the DOR and the second-order quadrupolar broadening in the MAS make interpretation of these more difficult. The comparison of 
the DAS to the DOR spectra immediately identifies the isotropic shifts and these are compiled in table 8.1 .

Figure 8.3 shows the DAS spectra of the various pyroxene silicates taken at $11.7 \mathrm{~T}$ (67.797 MHz). No DOR spectra were performed for these compound at this field. MAS experiments were conducted for all compounds though only the MAS of diopside at 11.7T is shown in figure 8.5. The signal-to-noise ratio of the clinoenstatite spectrum was significantly worse than in the previous experiments at $9.4 \mathrm{~T}$.

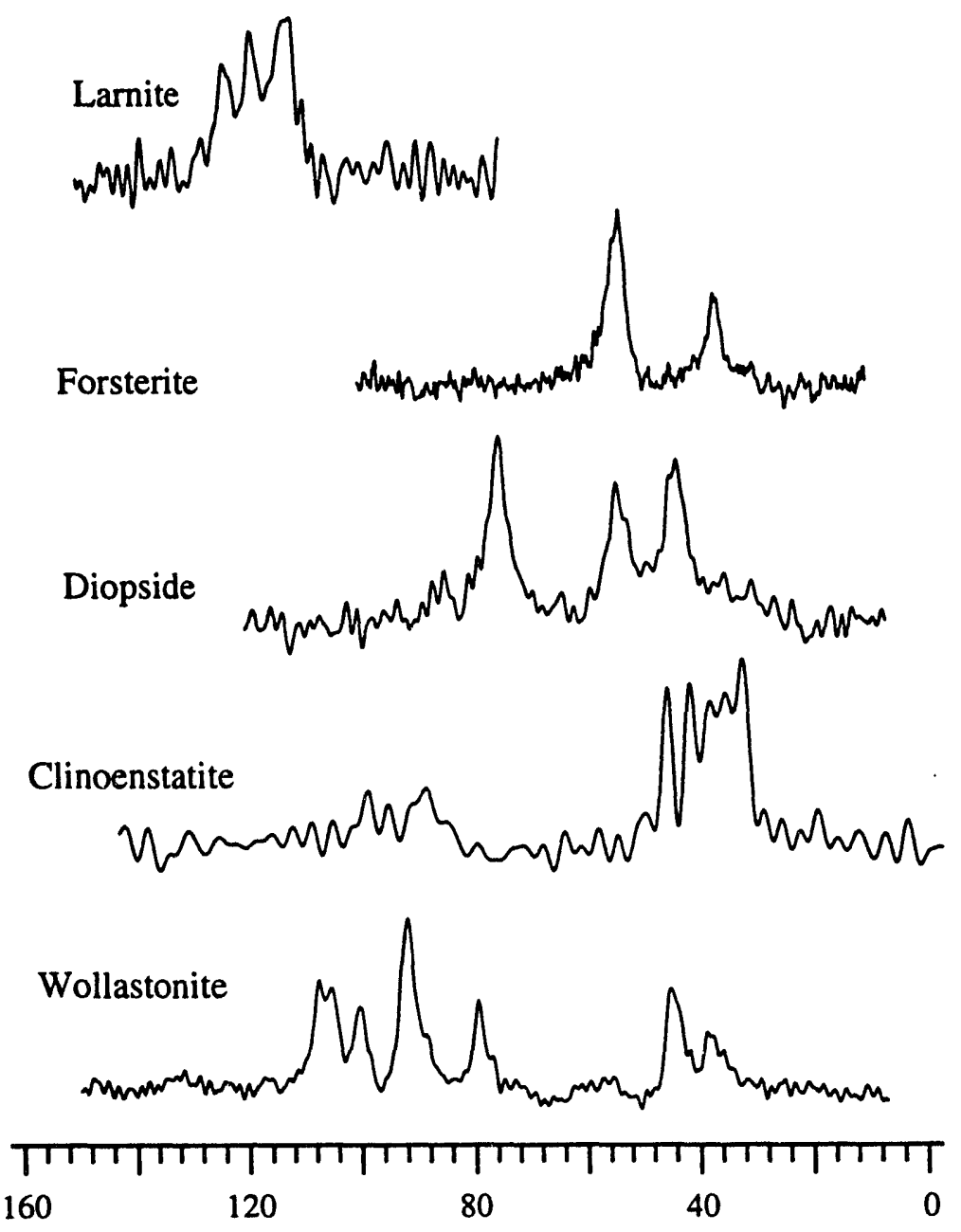

Frequency (ppm from $\mathrm{H}_{2}{ }^{17} \mathrm{O}$ )

Figure 8.3 Crystalline 11.7T DAS Spectra. All spectra are shown on the same scale referenced relative to $\mathrm{H}_{2}{ }^{17} \mathrm{O}$. The isotropic peaks in the clinoenstatite and larnite spectra were determined by performing these experiments twice, however the signal to noise dictates that the errors in the measured isotropic shifts will be approximately twice (or \pm 2 ppm) those in the other spectra. 
This is probably due to the fact that some of the sample was lost over the course of these and other experiments and therefore the overall signal was significantly reduced. Both the clinoenstatite and larnite DAS experiments were performed twice and peaks appeared at the positions given in table 8.1 in both sets of experiments. In the case of wollastonite, the number of resolved peaks is reduced from six non-bridging and two bridging sites at 9.4 $\mathrm{T}$ to five non-bridging and two bridging sites at $11.7 \mathrm{~T}$. The most intense bridging peak again is the sum of two sites, just as in the 9.4T experiment. Also, the most intense non-bridging peak (at $92 \mathrm{ppm}$ ) is the sum of two sites with different quadrupolar coupling constants which are apparently crossing meaning that the isotropic chemical shift of the one with the larger quadrupolar coupling constant is greater than the isotropic chemical shift of the other, leading to the possibility that at a given field they will have identical total isotropic shifts. If this experiment could be performed at a field as high as $14 \mathrm{~T}$, this peak would probably again split into two peaks.

The isotropic shifts at both $9.4 \mathrm{~T}$ and $11.7 \mathrm{~T}$, as well as the calculated isotropic chemical shifts and quadrupolar coupling product, $P_{Q}$ are listed in table 8.1 for each of the compounds studied. There is some ambiguity as to the assignment of the peaks in the wollastonite spectra between the two fields, however it is reasonable that the quadrupolar coupling constants should be relatively similar in both the bridging and the non-bridging region. Thus the order of the peaks should not change significantly. It may be shown that changing the order (and therefore the assignment in table 8.1) of any of the $11.7 \mathrm{~T}$ peaks will dramatically affect at least 2 of the isotropic shifts and coupling products. Thus we feel that the assignments below are quite reasonable. 


\begin{tabular}{|lcccc|}
\hline Compound & $\delta_{\text {obs }}^{9.4 T}(\mathrm{ppm})$ & $\delta_{o b s}^{11.7 T}(\mathrm{ppm})$ & $\delta_{i s o, c s}(\mathrm{ppm})$ & $P_{Q}(\mathrm{MHz})$ \\
& 117.3 & 123.3 & 133.9 & $2.9 \pm 0.2$ \\
Larnite & 113.3 & 118.5 & 127.8 & $2.7 \pm 0.2$ \\
$\left(\mathrm{Ca}_{2} \mathrm{SiO}_{4}\right)$ & 108.8 & 113.4 & 121.6 & $2.5 \pm 0.2$ \\
& 106.3 & 112.0 & 122.1 & $2.8 \pm 0.2$ \\
& & & & \\
Diopside & 69.2 & 75.1 & 85.6 & $2.8 \pm 0.2$ \\
(MgCaSi$\left._{2} \mathrm{O}_{6}\right)$ & 48.5 & 54.0 & 63.8 & $2.7 \pm 0.2$ \\
& 28.6 & 43.3 & $69.5(\mathrm{~b})$ & $4.5 \pm 0.1$ \\
& & & & \\
& 39.3 & 45.5 & 56.6 & $2.9 \pm 0.3$ \\
Clinoenstatite $_{\left(\mathrm{MgSiO}_{3}\right)}$ & 34.5 & 44.1 & 61.2 & $3.6 \pm 0.3$ \\
& 32.3 & 42.0 & 59.3 & $3.6 \pm 0.3$ \\
& 26.3 & 39.0 & 61.7 & $4.2 \pm 0.3$ \\
& 18.0 & 36.8 & $70.3(\mathrm{~b})$ & $5.1 \pm 0.2$ \\
& 15.0 & 34.7 & $69.8(\mathrm{~b})$ & $5.2 \pm 0.2$ \\
Forsterite & & & & \\
$\left(\mathrm{Mg}_{2} \mathrm{SiO}_{4}\right)$ & 49.0 & 57.1 & 71.5 & $3.3 \pm 0.3$ \\
& 49.0 & 54.8 & 64.3 & $2.7 \pm 0.3$ \\
& 30.8 & 37.5 & 49.4 & $3.0 \pm 0.2$ \\
& & & & \\
& 103.4 & 107.4 & 114.5 & $2.3 \pm 0.2$ \\
Wollastonite $_{\left(\mathrm{CaSiO}_{3}\right)}$ & 100.1 & 105.1 & 114.0 & $2.6 \pm 0.2$ \\
& 96.5 & 100.2 & 106.8 & $2.3 \pm 0.2$ \\
& 89.0 & 91.9 & 97.1 & $2.0 \pm 0.2$ \\
& 85.8 & 91.9 & 102.8 & $2.9 \pm 0.2$ \\
& 74.3 & 79.3 & 88.2 & $2.6 \pm 0.2$ \\
& 28.2 & 44.9 & $74.6(\mathrm{~b})$ & $4.8 \pm 0.1$ \\
& 28.2 & 44.9 & $74.6(\mathrm{~b})$ & $4.8 \pm 0.1$ \\
& 21.6 & 37.8 & $66.6(\mathrm{~b})$ & $4.7 \pm 0.1$ \\
\hline
\end{tabular}

Table 8.1 Isotropic Chemical Shifts and Quadrupolar Coupling Products from Two Field Studies. The isotropic shifts measured from the spectra in figure 8.2 and 8.3 were used to compute the isotropic chemical shifts and quadrupolar products for all of the oxygen sites in each of the six minerals. The isotropic shifts marked with a (b) indicated bridging oxygen sites. The errors for the observed isotropic shifts were $\pm 1 \mathrm{ppm}$ (except for larnite and clinoenstatite at $11.7 \mathrm{~T}$ which had $\pm 2 \mathrm{ppm}$ errors) providing an isotropic chemical shift error of $\pm 2 \mathrm{ppm}$ ( $\pm 3 \mathrm{ppm}$ for larnite and clinoenstatite).

If the spectra could be collected at yet a third field (i.e. $<7 \mathrm{~T}$ or $>14 \mathrm{~T}$ ) then these assignments may become more clear. To calculate the isotropic chemical shifts and quadrupolar coupling products, the coupled equations 8.3 were solved. The errors in the calculated parameters were computed using standard error propagation techniques. 
In addition to these quadrupolar parameters, the magic angle spinning spectra of diopside at $9.4 \mathrm{~T}$ and $11.7 \mathrm{~T}$ were simulated using automated simplex routines. By effectively fixing the coupling product and the isotropic chemical shift values we were able to generate highly accurate values for the asymmetry parameters. This in turn allows us to recover the real quadrupolar coupling constant $C_{Q}$ which is proportional to the field gradient in the z-direction of the principal axes system of the nucleus. The experimental spectra and best fit simulations are shown in figure 8.4. The values we extracted agreed quite well with previous work. ${ }^{150}$

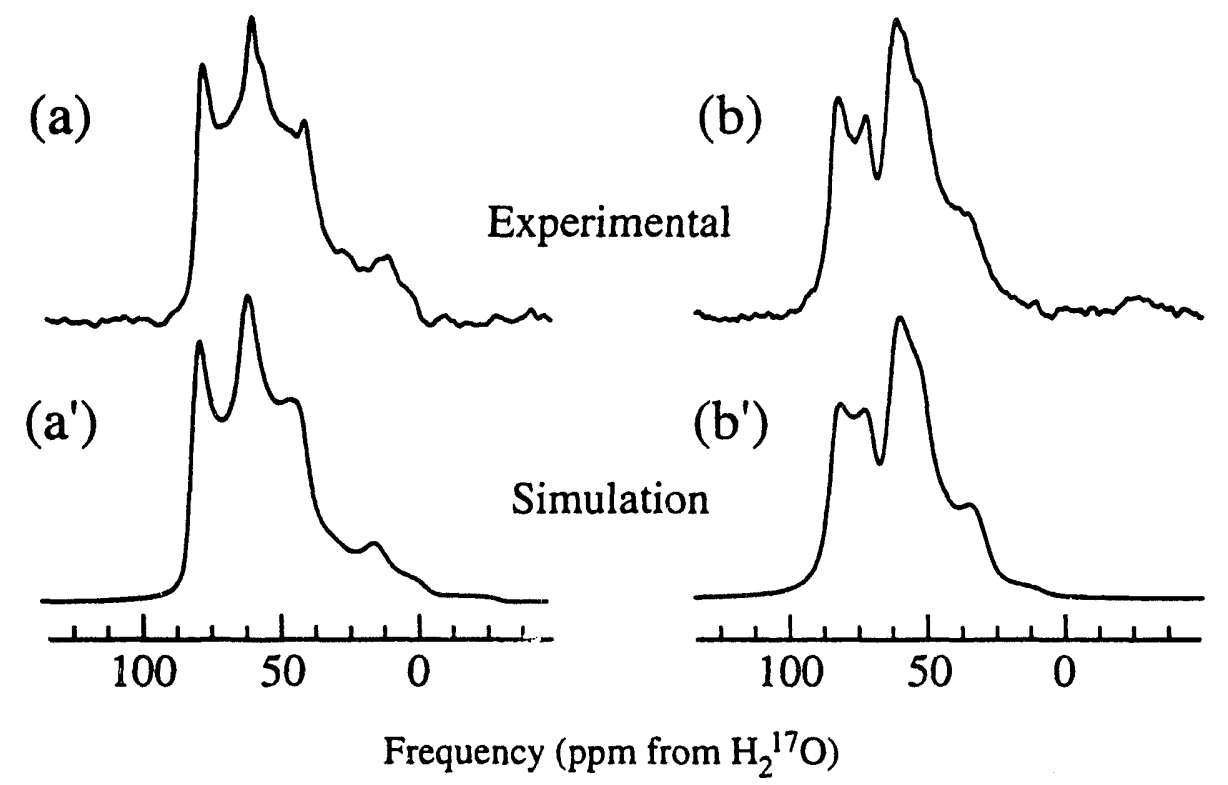

Figure 8.4 Crystalline Diopside 9.4T and 11.7T MAS Spectra. The MAS spectra at 9.4T (a) are shown as well as at $11.7 \mathrm{~T}$ (b). All spectra are shown with a ppm scale referenced relative to $\mathrm{H}_{2}{ }^{17} \mathrm{O}$. The simulations $\left(a^{\prime}\right.$ and $\left.b^{\prime}\right)$ were performed with the isotropic chemical shift and quadrupolar product parameters fixed to those in table 8.1. The simulation results are given in table 8.2.

In table 8.2 below, the values extracted from the simulations of the diopside MAS spectra in figure 8.4 are compiled. Also shown are the parameters reported by Timken et al. ${ }^{150}$ previously from single field diopside MAS simulations. There are no error bars for the Timken results, however, since these fits were done by hand without least-squares minimization. Our results include actual error bars since the MINUIT subroutine calculates and uses gradietts on the chi squared surface. 


\begin{tabular}{|cccc|ccc|}
\hline \multicolumn{4}{|c|}{ This Study } & \multicolumn{3}{c|}{ Timken et al. $^{150}$} \\
Site & $\delta_{\text {iso,cs }}(\mathrm{ppm})$ & $C_{Q}(\mathrm{MHz})$ & $\eta_{Q}$ & $\delta_{\text {iso,cs }}(\mathrm{ppm})$ & $C_{Q}(\mathrm{MHz})$ & $\eta_{Q}$ \\
\hline 1 & $86 \pm 1$ & $2.83 \pm 0.05$ & $0.13 \pm 0.10$ & 84 & 2.7 & 0.0 \\
2 & $64 \pm 1$ & $2.74 \pm 0.05$ & $0.00 \pm 0.10$ & 63 & 2.7 & 0.1 \\
3 & $69 \pm 1$ & $4.39 \pm 0.05$ & $0.36 \pm 0.05$ & 69 & 4.4 & 0.3 \\
\hline
\end{tabular}

Table 8.2 Diopside Quadrupolar Parameters. These quadrupolar parameters were extracted by simultaneously fitting the MAS spectra in figure 8.4. The error bars are indicated in the table.

In the three chain silicates studied (diopside, clinoenstatite, and wollastonite), the occupancy of terminal oxygen sites in the structure is twice that of bridging oxygen species. In forsterite and larnite, all oxygen sites are non-bridging. Diopside, clinoenstatite, and wollastonite have three, six, and nine crystallographically distinct oxygen sites, respectively, and one, two, and three different bridging sites respectively. Referring to table 8.1, we note that the quadrupolar products $P_{Q}$ for the oxygen sites in the chain silicates are predominantly less than $4 \mathrm{MHz}$. Values higher than $4.3 \mathrm{MHz}$ follow a 1:2:3 ratio, respectively, for diopside, clinoenstatite, and wollastonite, suggesting that lines associated with these values should be assigned to bridging sites. This observation is compatible with the results of Oldfield and coworkers in their studies of these and similar silicates $^{150}$. The DAS technique now allows complete resolution of all sites, even in wollastonite. For this silicate with nine crystallographic oxygen sites, the NMR data now reveal six distinct terminal sites as well as two inequivalent bridging sites occurring in a 2:1 ratio.

A number of trends are conspicuous when the isotropic chemical shifts for the various types of oxygen sites are examined. All of the bridging sites have isotropic chemical shifts to within $4 \mathrm{ppm}$ of $71 \mathrm{ppm}$, referenced to the single oxygen-17 resonance from $\mathrm{H}_{2}{ }^{17} \mathrm{O}$. This is an extremely small deviation considering the wide range of chemical shifts which have been reported for oxygen-17. ${ }^{158}$ Here, however, all of the oxygen 
sites are quite similar and differ only in the identities of neighboring cations. As noted by Oldfield and coworkers, ${ }^{150}$ the chemical shifts of the bridging oxygen atoms is generally less sensitive to the nature of the nearby cations due to the distance separating the oxygen nuclei and the cations.

Chemical shifts of $\mathrm{Si}-\mathrm{O}$ terminal sites are more strongly dependent on the cations present. The deshielding of the oxygen nucleus as the effective ionic radius of the cation increases has been established empirically ${ }^{150}$ and when the cations are magnesium ions (as in forsterite and clinoenstatite), the isotropic chemical shifts calculated range between 49 and $72 \mathrm{ppm}$. When calcium ions are present exclusively (wollastonite and larnite), the isotropic chemical shifts for the ten sites lie between 88 and $134 \mathrm{ppm}$. In the mixed cation compound (diopside), both terminal oxygen sites had intermediate chemical shift values (64 and $88 \mathrm{ppm}$ ). Thus it appears that each oxygen in diopside experiences an averaged chemical shift value from the surrounding cations.

Similar trends are also observed when the quadrupolar coupling products are examined. For the bridging sites, $P_{Q}$ values range between 4.5 and $5.2 \mathrm{MHz}$. This again is a very small range considering that oxygen-17 coupling constants as large as $12 \mathrm{MHz}$ are observed for sites with similar coordination or stoichiometry ${ }^{159}$. For the terminal sites in the magnesium-containing minerals $P_{Q}$ values from 2.8 to $4.2 \mathrm{MHz}$ are found. For similar sites near calcium cations the experimentally determined values are generally lower and fall between 2.0 and $2.9 \mathrm{MHz}$. Since the electronegativities of both cations are quite similar, the electric field gradients near these ions are only slightly dependent on the type of ion itself. The quadrupolar coupling products for terminal sites in diopside, which both fall close to the overlap point of the ranges for the two types of cations, tend to support the argument that an average environment is experienced at these sites. A more noticeable difference is between the bridging and terminal oxygen quadrupolar environments since the field gradients at bridging sites are almost double those at terminal sites. 
Finally, from the MAS simulations at the two fields, the values for the asymmetry parameter, $\eta_{Q}$, at each of the three sites in diopside were determined (table 8.2). This provides additional information above and beyond the coupling product, $P_{Q}$. It also allows us to determine with greater precision the value of the actual quadrupolar coupling constants $C_{Q}$, giving a quantitative description of the strength the field gradient at each site. Further, all sites in diopside have asymmetry parameters near zero, indicating that the $\mathrm{x}$ and $\mathrm{y}$ gradients are of approximately the same strength. The asymmetry parameter of the bridging oxygen also may be correlated with the bridging $\mathrm{Si}-\mathrm{O}-\mathrm{Si}$ bond angle determined from the crystal structure (see the next section). When many such bond angle/asymmetry parameter correlations have been determined, this information may be used to determine an unknown bond angle from quadrupolar parameters. ${ }^{46}$

In conclusion, we have shown that by performing field-dependent DAS experiments on oxygen-17 in minerals, parameters are obtained which can be directly correlated with structural information. Trends are recognized in the isotropic chemical shifts and the quadrupolar coupling strengths for a series of silicate minerals. It has been demonstrated that these parameters depend on the type of oxygen crystallographic site and the neighboring cation present in the crystals, corroborating extensive earlier studies but further providing information on all oxygen sites present in certain complex silicate minerals.

\section{Amorphous Silicates}

The difference between a glass and a crystal lies in disorder present in the intermediate-range glass structure that eliminates long-range translational symmetry (see figure 8.5). Characterization of disorder is an important experimental objective because it is a critical test of the accuracy of models of glass structure. In pure silica glasses $\left(\mathrm{SiO}_{2}\right)$, the basic building block is the $\mathrm{SiO}_{4}$ tetrahedron which form a three-dimensional network with the overall disorder coming in the range of $\mathrm{Si}-\mathrm{O}-\mathrm{Si}$ bond angles made by joining 
tetrahedra at the corners. ${ }^{160}$ This distribution of bond angles in $\mathrm{SiO}_{2}$ has been extensively studied with both X-ray and NMR techniques. ${ }^{67,161-167}$

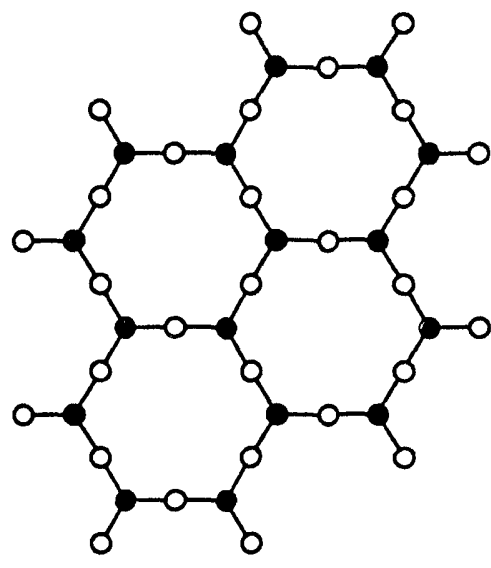

a)

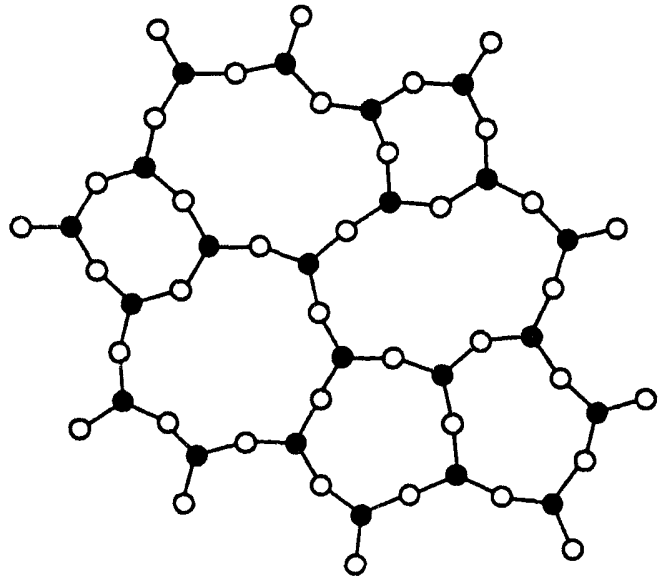

b)

Figure 8.5 Comparison of $\mathrm{A}_{2} \mathrm{O}_{3}$ Crystal and Glass Lattice Structures. The two structures represent possible planar configurations for a sample with $\mathrm{A}_{2} \mathrm{O}_{3}$ stoichiometry. The dark circles represent $A$ atoms while the open circles represent oxygen atoms. Notice that the $\mathrm{AO}_{3}$ building block used in both structures preserves basically identical A-O bond lengths and $\mathrm{O}-\mathrm{A}-\mathrm{O}$ bond angles. The primary difference lies in the distribution of $\mathrm{A}-\mathrm{O}$ A bond angles leading to the wide range of rings in the glass as opposed to the strict $180^{\circ}$ bond angle with six sided rings in the crystal.

Figure 8.5 shows a possible planar structure for both a crystal and a glass with the $\mathrm{A}_{2} \mathrm{O}_{3}$ stoichiometry (for example $\mathrm{B}_{2} \mathrm{O}_{3}$ ). This figure could be thought of as a two-dimensional analog of the three-dimensional lattices formed from $\mathrm{AO}_{2}$ glasses (such as $\mathrm{SiO}_{2}$ ). It is immediately apparent that in the crystal, the A atoms are always surrounded by three oxygen atoms with strict $120^{\circ}$ bond angles; each $O$ atom forms a distinct $180^{\circ}$ bond as well. The glass structure maintains the basic $\mathrm{AO}_{3}$ building block with $120^{\circ}$ bonds, however now the connecting A-O-A bonds are no-longer $180^{\circ}$. With only a very slight increase in the overall energy of the crystal structure, the glass structure may be formed. This indicates some of the basic local order trends seen in three dimensional glasses. 


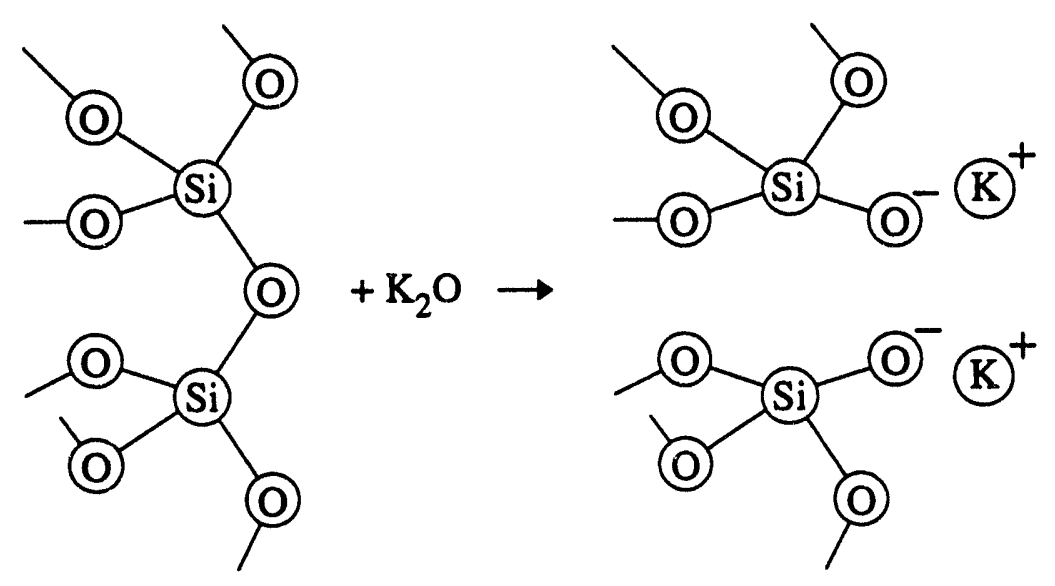

Figure 8.6 Insertion of Modifying Cations into Silicate Glasses. The insertion of a $\mathrm{K}_{2} \mathrm{O}$ "molecule" into the $\mathrm{SiO}_{2}$ glass lattice causes the creation of two non-bridging oxygen atoms from a bridging oxygen. The distribution of these modifying cations throughout the glass may proceed in either a random or partially random fashion as the modifying cation concentration is increased.

In network-modified silicate glasses, the continuous disordered network of $\mathrm{SiO}_{4}$ tetrahedra is presumed to be disrupted by modifying cations which create non-bridging oxygen atoms (oxygen atoms bonded to only one silicon atom). ${ }^{168}$ Figure 8.6 shows how the addition of a modifying cation (in this case in the form of $\mathrm{K}_{2} \mathrm{O}$ ) creates non-bridging oxygen sites in a silicate glass. Two principal sources of disorder are thought to be this disruption of the network and the distribution of bond angles ( $\mathrm{Si}-\mathrm{O}-\mathrm{Si}$ ) between network forming cations (as mentioned previously). It is well established experimentally that the silicon and oxygen are ordered locally in network modified-silicate glasses, and that the $\mathrm{SiO}_{4}$ tetrahedra remain the basic structural unit. From extended $\mathrm{X}$-ray absorption fine structure (EXAFS) studies of modified cations, we know that they too are regularly coordinated by oxygen, ${ }^{169,170}$ having coordination polyhedra and bond lengths similar to those in crystalline silicates. Isotopically substituted neutron scattering has also shown that ordering associated with modifier cations extends beyond the first coordination splere, by detecting strong correlations between $\mathrm{Ca}-\mathrm{Ca}$ as well as $\mathrm{Ca}-\mathrm{O}$ distances in $\mathrm{CaSiO}_{3}$ glass ${ }^{171,172}$. This is consistent with ${ }^{29} \mathrm{Si}$ NMR studies of silicate glasses, which show that the distribution of non-bridging oxygen atoms is not random, being close to binary 165,173 ; the deviation from a binary distribution depends on the electronegativity of 
the network modifier and on the glass transition temperature. Taken together, these experimental data indicate considerable order associated with network modification. Quantification of the remaining disorder associated with variations in bridging angles between network-forming cations is therefore important. ${ }^{166,167}$

Volumetrically, silicate glasses are dominated by oxygen anions, yet despite this, the structure of silicate glasses has been studied almost entirely from the perspective of the cations and their coordination. For example, $\mathrm{X}$-ray scattering experiments are most sensitive to scattering from cations (network-forming and network-modifying) that are heavier than oxygen anions, EXAFS experiments concentrate on network modifiers such as $\mathrm{Na}^{+}$or $\mathrm{Ca}^{2+}$, and ${ }^{29} \mathrm{Si} \mathrm{NMR}$ experiments specifically observe signal from the networkforming cation. In this section, we investigate the local environments of the oxygen anions. As oxygen is the connecting atom between locally ordered tetrahedral environments, the intermediate-range disorder in the glass will be reflected in the range of environments exhibited by these oxygen atoms. As shown in the previous section, ${ }^{17} \mathrm{O} N \mathrm{NMR}$ is a sensitive and direct way to characterize these interconnections. Previously, ${ }^{17} \mathrm{O}$ NMR has been used to study glasses with conventional static and MAS techniques. ${ }^{159,174}$ These methods of course are seriously hampered by both the anisotropic broadening arising from a range of crystallite orientations and the distribution of local environments in a glass which lead to a continuum of sites. These two contributions to the lineshape may not be separated in an experiment such as MAS, since this fails to remove all of the anisotropic broadening arising from the second-order quadrupolar interaction (see cnapter 2). In contrast, the two-dimensional DAS experiment is well suited for this type of system, as this may be used to correlate high-resolution isotropic peaks in one dimension (which will be a broad distribution in a glass due to the continuum of sites) with the individual powder patterns for each site in the second dimension (see chapter 3). 


\section{Experimental}

All of the spectra of silicate glasses in this section were taken with one of two types of home built probes designed by Mueller et al..$^{51}$ and Eastman et al. ${ }^{44}$ The usual field strength was either $9.4 \mathrm{~T}$ or $11.7 \mathrm{~T}$ to give the highest initial polarization and therefor highest signal-to-noise ratios, since individual slices from the 2D DAS spectra were to be simulated. Additionally, the HyperSEDAS or SEDAS (see chapter 4) pulse sequences were used since both the transverse and longitudinal ( $T_{2}$ and $\left.T_{1}\right)$ relaxation times for these samples were in general quite long (1-20 seconds). The pulse widths were usually between 3 and $7 \mu$ s and the rotor reorientation times were often less than $40 \mathrm{~ms}$. For the shifted-echo experiments, the DAS echo was usually shifted from 4 to 8 rotor periods out in time (approximately $1 \mathrm{~ms}$ ). The spinning rate was from 5 to $7 \mathrm{kHz}$ and the $k=1$ angle pair was used for most experiments. In all glass spectra the time domain data in the $t_{1}$ dimension rapidly decayed away due to the broad distribution of sites in the isotropic dimension and therefore usually only 40-70 total points were collected in this dimension. In the second dimension, usually 256 or 512 points were taken to provide the necessary digital resolution to see distinct features in the anisotropic powder patterns.

The ${ }^{17} \mathrm{O}$ labeled glasses were again prepared by Stebbins and coworkers. The usual enrichment was between 35 and 50 percent and was achieved by the addition of ${ }^{17} \mathrm{O}$ labeled water to $\mathrm{SiCl}_{4}$ to produce isotopically labeled $\mathrm{SiO}_{2}$ which was then used to make the glasses by combination with alkaline and alkaline earth metal oxides (often ${ }^{17} \mathrm{O}$ labeled as well). In general the glasses were quenched from the liquid state at about $1100^{\circ}$ to $1600^{\circ} \mathrm{C}$ in a vacuum oven to assure that no oxygen was lost or exchanged in the glass formation. In some cases, the samples were sealed in a $\mathrm{Pt}$ tube to allow quenching from even higher temperature than were possible in the vacuum oven (specifically this applies to the amorphous $\mathrm{SiO}_{2}$ ). In all cases, stoichiometry and phase were tested with both ${ }^{17} \mathrm{O}$ and ${ }^{29}$ Si MAS NMR at Stanford before attempting DAS experiments. For more details of the synthesis see the thesis by Chmelka. ${ }^{47}$ 


\section{Amorphous Silica $\left(\mathrm{SiO}_{2}\right)$}

The study of amorphous $\mathrm{SiO}_{2}$ with ${ }^{17} \mathrm{O}$ DAS represents the natural starting point for a discussion of silicate glasses (as a side note, chronologically this was not the first glass studied with DAS, however with the clarity of hindsight, this represents a more logical place to begin the discussion of silicate glasses). A range of different silica samples were prepared by Stebbins and coworkers for study with DAS as well as some from Dupree and coworkers. In all cases the spectra were very similar for all kinds of silica glasses. Figure 8.7 shows the 2D DAS spectrum of amorphous $\mathrm{SiO} 2$ taken at $9.4 \mathrm{~T}$ with $k=1\left(37.38^{\circ}\right.$ and $79.19^{\circ}$ angle pair).

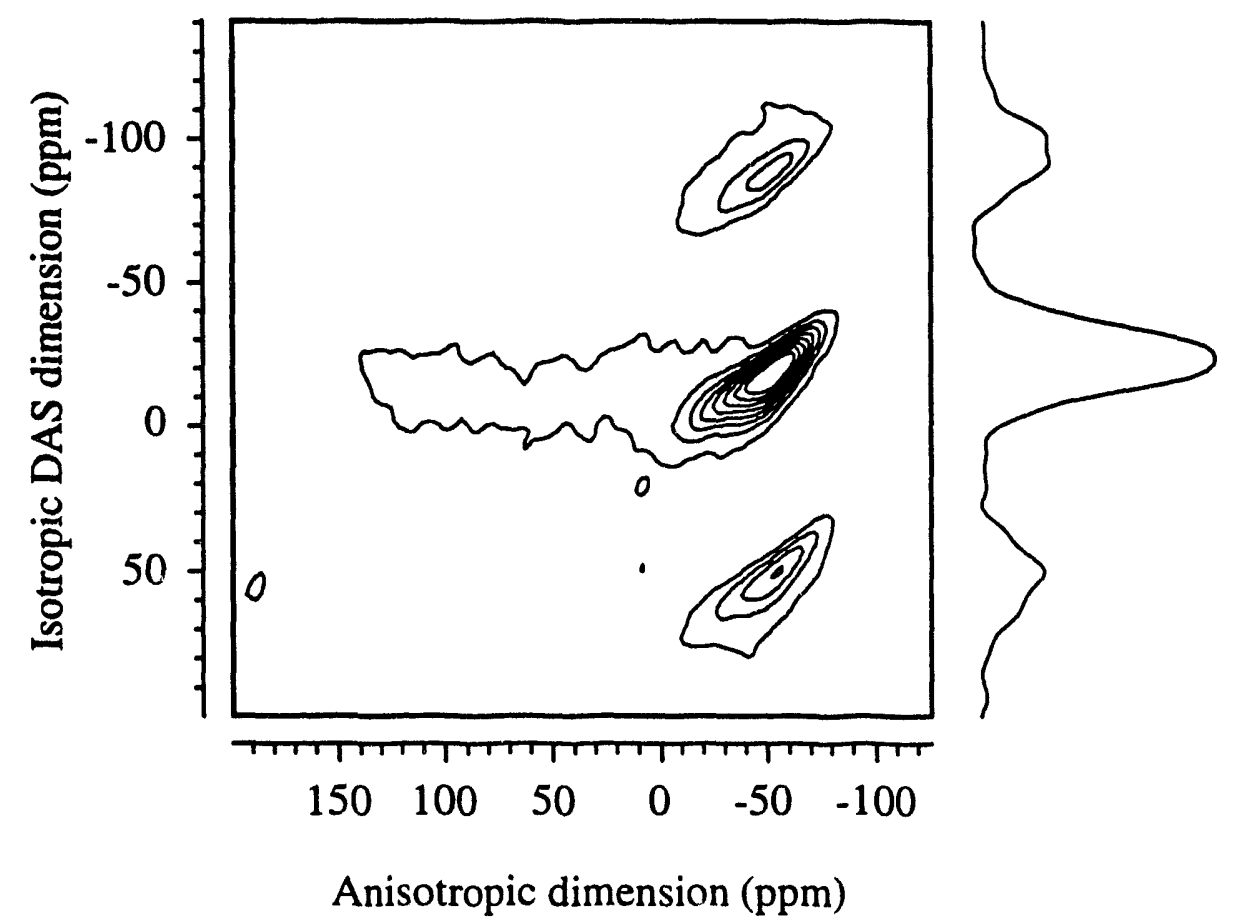

Figure 8.7 2D DAS Spectrum of Amorphous $\mathrm{SiO}_{2}$. The isotropic dimension shows a single broad peak with spinning sidebands on both sides. The anisotropic slices are seen to increase in asymmetry parameter and decrease in quadrupolar coupling as the isotropic shift gets larger.

Notice that the center band is quite broad (approximately $20 \mathrm{ppm}$ full width at half maximum, FWHM) and the sidebands which appear to either side are fairly strong due to the large quadrupolar coupling in this sample (quadrupolar coupling constant of about 5 to $6 \mathrm{MHz}$ ). Also, the overall shape of the anisotropic slices changes as the isotopic shift 
is increased. On the low frequency side of the isotopic peak, the asymmetry parameter for anisotropic slices is nearly zero. As the isotropic shift increases in frequency, the asymmetry parameter gets larger and the quadrupolar coupling constant gets smaller. This is observed in both this data and the data from 11.7T. Table 8.3 compiles the intensity, isotropic shift, quadrupolar coupling constant and asymmetry parameter for each of the 18 slices with significant intensity (slices which may be simulated) through the centerband isotropic peak. The slice number corresponds to the absolute number of the slice through $\omega_{1}$ (for this data set, the $t_{1}$ dimension was zero filled to 256 points).

\begin{tabular}{ccccc||ccccc}
\hline Slice & $C_{Q}$ & $\eta_{Q}$ & Pop. & $\delta_{\text {iso,cs }}$ & Slice & $C_{Q}$ & $\eta_{Q}$ & Pop. & $\delta_{\text {iso,cs }}$ \\
\hline 109 & 5.85 & 0.00 & 0.24 & 32.5 & 118 & 5.78 & 0.13 & 0.85 & 55.0 \\
110 & 6.00 & 0.00 & 0.44 & 40.2 & 119 & 5.70 & 0.15 & 0.79 & 55.5 \\
111 & 6.14 & 0.00 & 0.55 & 47.4 & 120 & 5.61 & 0.17 & 0.71 & 55.3 \\
112 & 6.18 & 0.02 & 0.65 & 51.4 & 121 & 5.53 & 0.18 & 0.61 & 55.7 \\
113 & 6.18 & 0.03 & 0.75 & 54.3 & 122 & 5.47 & 0.19 & 0.50 & 57.0 \\
114 & 6.11 & 0.05 & 0.83 & 54.6 & 123 & 5.43 & 0.20 & 0.40 & 59.0 \\
115 & 6.02 & 0.07 & 0.87 & 54.0 & 124 & 5.38 & 0.22 & 0.31 & 60.5 \\
116 & 5.93 & 0.09 & 0.89 & 54.0 & 125 & 5.12 & 0.26 & 0.22 & 55.6 \\
117 & 5.85 & 0.11 & 0.89 & 54.3 & 126 & 4.58 & 0.34 & 0.15 & 43.4 \\
\hline
\end{tabular}

Table 8.3 $\mathrm{SiO}_{2}$ Anisotropic Slice Fits. The simulations were performed using the computer programs in the appendix with the assumption that the chemical shift anisotropy was negligible.

It may be noted here that slices $109,110,125$ and 126 have values for either the quadrupolar coupling or isotropic chemical shift which do not follow the trends observed throughout the rest of the table. This is due to the fact that the signal-to-noise ratio of these slices made simulation difficult and these values are to be given much less significance than in the reşion of the peak (slices 116 and 117). Five of these slices (as well as 


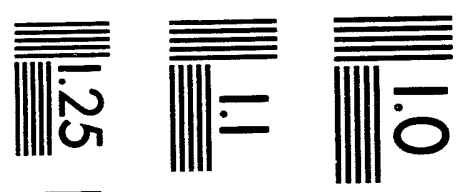

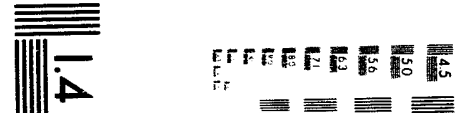

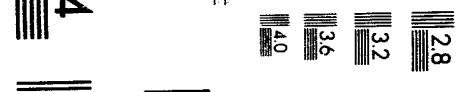

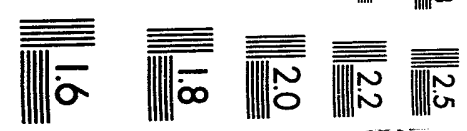



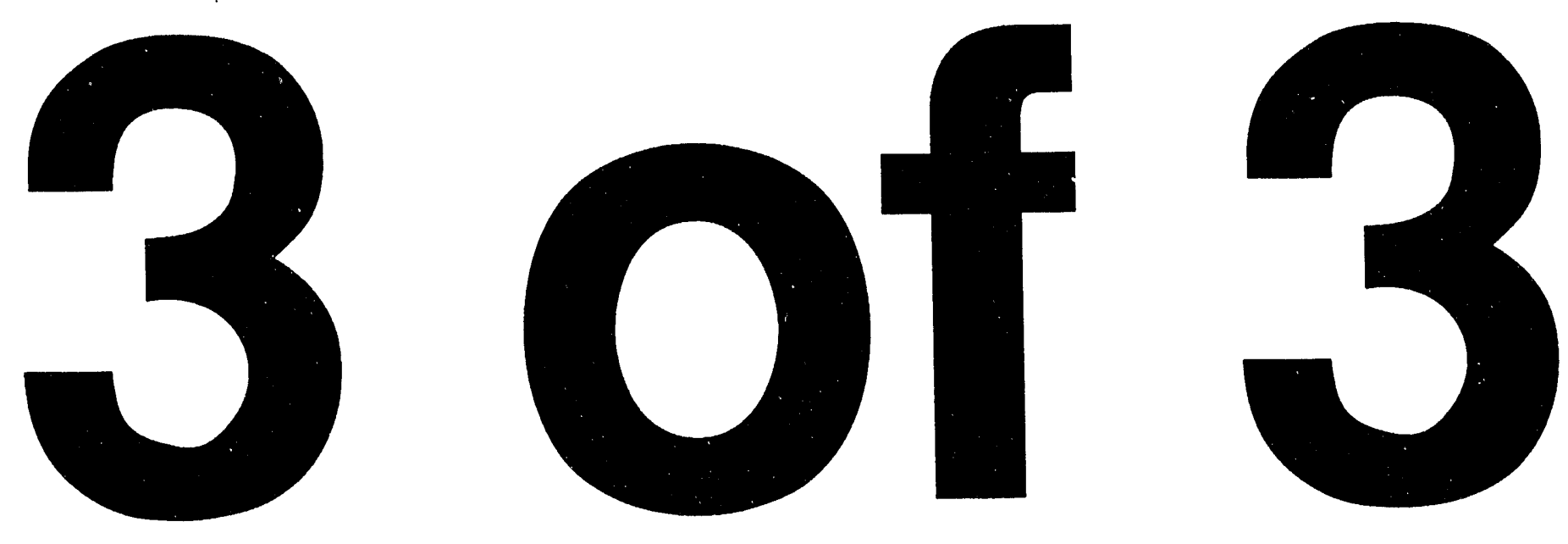


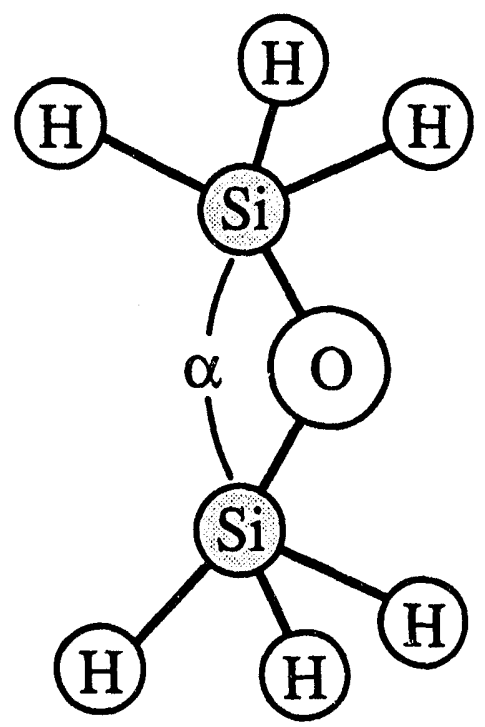

igure 8.9 Electric Field Gradient Model Compound;. The molecule $\mathrm{H}_{3} \mathrm{Si}-\mathrm{O}-\mathrm{SiH}_{3}$ may be used as a rough model to show the relative sizes of the EFG tensor as the Si-O-Si bond angle is changed.

As this bond angle is reduced, the $x$-axis becomes defined by the plane made by the two silicon atoms and the oxygen atom (the $y$-axis is of course perpendicular to the $x$ - and $z$ axes). At an angle of less than $180^{\circ}$, the $x$ and $y$ field gradients will no longer by identical and by definition (see equation 2.37) $\eta_{\mathrm{Q}}$ will be greater than zero. With simple point charge or electron bonding models it is impossible to assess accurately how rapidly the asymmetry parameter will grow towards the maximum possible value of one. Tossell and Lazzeretti have done a more thorough analysis of this molecule using modern $a b$ initio molecular orbital calculation algorithms ${ }^{142}$. Figure 8.10 shows the characteristic quadrupolar coupling constants and asymmetry parameters calculated for this molecule when the Si-O-Si bond angle was $180^{\circ}, 160^{\circ}$ and $140^{\circ}$. 

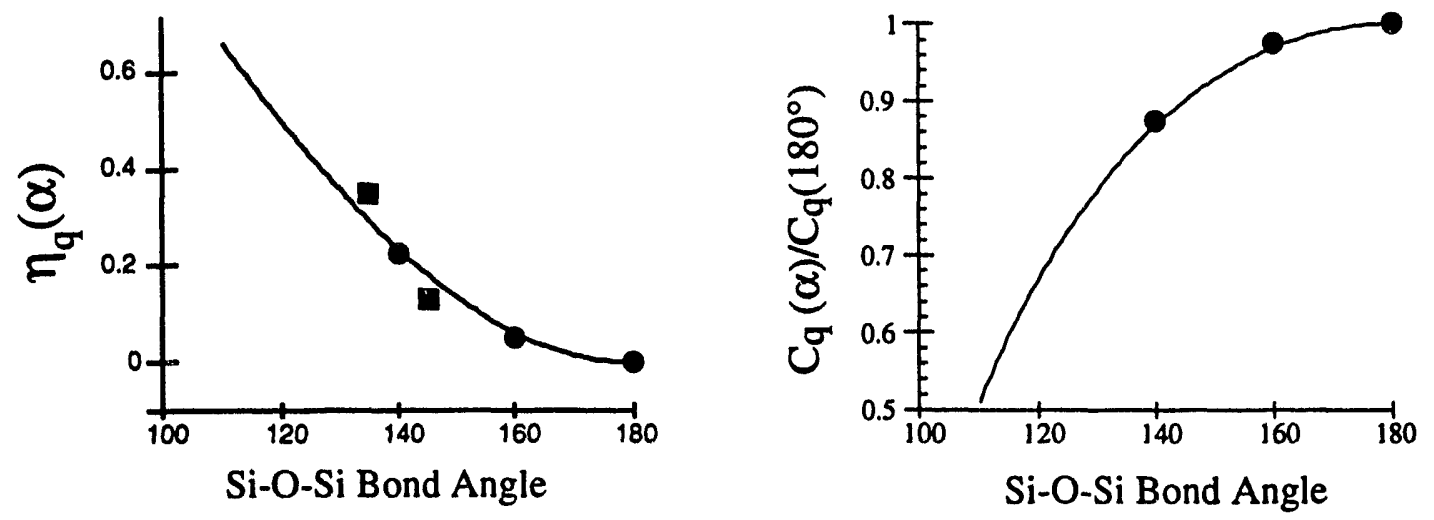

Figure 8.10 Ab Initio Quadrupolar Parameters for $\mathrm{H}_{3} \mathrm{Si}-\mathrm{O}-\mathrm{SiH}_{3}$. The quadrupolar coupling constant and asymmetry parameter were calculated using Gaussian algorithms for the angles $180^{\circ}, 160^{\circ}$ and $140^{\circ}$. Circles indicate values calculated by Tossell and Lazzeretti while square are experimental points from Stebbins $e t$ al.

The lines going through the points in these figures correspond to an empirical fit with the functions given below.

$$
\begin{aligned}
& C_{Q}(\angle S i-O-S i)=C_{Q}\left(180^{\circ}\right) \frac{2 \cos (\angle S i-O-S i)}{\cos (\angle S i-O-S i)-1} \\
& \eta_{Q}(\angle S i-O-S i)=1-\cos (\angle S i-O-S i)
\end{aligned}
$$

These equations describe Tossell and Lazzeretti's data reasonably well. The two squares in figure 8.10 indicate asymmetry parameters measured from simulations of MAS spectra of wadeite $\left(134.7^{\circ}\right.$ bridging bond angle) and cristobalite ${ }^{175}\left(146.4^{\circ}\right.$ bond angle). Notice that these fall very near the empirical asymmetry parameter curve.

The equation used to describe the asymmetry parameter in terms of the bridging bond angle may be inverted and used to convert our data in table 8.3 from intensity (or population) as a function of slice number into intensity as a function of bond angle. This involves two separate conversions. First, the data must be converted from intensity as a function of slice number (or isotropic shift) into intensity as a function of asymmetry parameter. This is equivalent to redefining the axis in the one dimensional isotropic spectrum from slice number (or ppm) into asymmetry parameter. This is not, however, a simple linear transformation and as such the intensity at each point must be rescaled by the gradient at that point. 


$$
I(\eta)=I(\delta)\left|\frac{d \eta}{d \delta}\right|
$$

The $\delta$ in this equation indicates the isotropic shift of a given slice while $\eta$ corresponds to the asymmetry parameter for that slice. These derivatives may be computed numerically by graphing the asymmetry parameter as a function of isotropic shift and empirically fitting the resulting curve. Additionally, the value of the asymmetry parameter may be extrapolated with an empirical curve to determine the bond angles in regions where the intensity is too low to simulate individual slices. The same procedure must be used to convert from intensity as a function of asymmetry parameter to intensity as a function of bridging bond angle, $\alpha$. This however is not as difficult since we know a functional form for the gradient already.

$$
I(\alpha)=I(\eta)\left|\frac{d \alpha}{d \eta}\right|=I(\eta) \sin \alpha
$$

This procedure leads to the bond angle distribution shown in figure 8.11 (reported first by Grandinetti et al. ${ }^{176}$ )

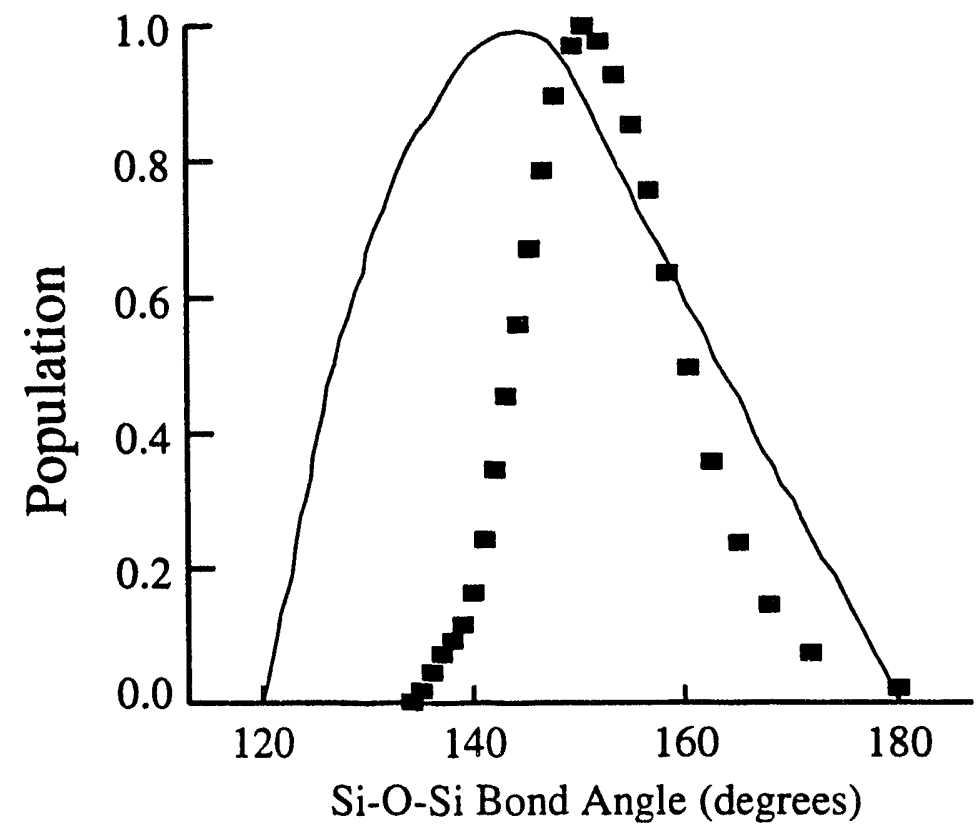

Figure 8.11 Amorphous $\mathrm{SiO}_{2}$ Bond Angle Distribution. The squares are the bond angle distribution arrived at from the DAS spectral analysis. The line indicates the bond angle distribution found by Mozzi and Warren with X-ray scattering experiments. 
The peak in this bond angle distribution occurs at approximately $150^{\circ}$. The absolute accuracy of this bond angle distribution is about $\pm 3^{\circ}$ at the peak and $\pm 10^{\circ}$ in the wings (where the simulations were of significantly reduced precision and extrapolation of the asymmetry parameters was used).

A second approach to determine the bond angle is to use the equation relating the quadrupolar coupling constant to bond angle (equation 8.4). To do this, we again must use the equivalent Jacobian equations to rescale the axes from intensity as a function of slice number (isotropic shift) to intensity as a function of bond angles.

$$
I(\alpha)=I\left(C_{Q}\right)\left|\frac{d C_{Q}}{d \alpha}\right|=I(\delta)\left(\frac{2 C_{Q}\left(180^{\circ}\right) \sin \alpha}{(\cos \alpha-1)^{2}}\right)\left|\frac{d \delta}{d C_{Q}}\right|
$$

This is slightly more difficult since we do not have an absolute value for the quadrupolar coupling constant at $180^{\circ}$. The simplest solution is to use the asymmetry parameter of the highest signal-to-noise ratio slice to determine the bond angle for that slice and use the empirical equation 8.4 to extrapolate to $C_{Q}\left(180^{\circ}\right)$. This requires that both empirical relations in equation 8.4 hold reasonably well, which may not be as accurate as in the case where only the asymmetry parameter i sused. However, when the quadrupolar coupling constants in table 8.3 are plotted against the isotropic shifts it is apparent that they form a nearly linear relationship which allows much greater confidence when extrapolating to the coupling constants in the outer slices. Also, the percent error bars on the quadrupolar coupling constants from the simulation are much smaller then those for the asymmetry parameters. Additionally, the linear relationship allows easy computation of the derivatives in equation 8.7. The bond angle distribution derived from this method is virtually identical to the one in figure 8.11. Also shown in figure 8.11 is the Si-O-Si bond angle distribution of Mozzi and Warren. ${ }^{161}$ Both the DAS and X-ray scattering bond angle distributions show a number of similarities. First, they both have a sharp cut off on the low angle side. This corresponds to the point where steric hindrances make the 
small bridging bond angles energetically unfavorable. Second, both have a long tail on the high angle side. This again is reasonable, since there will be no steric hindrances as the bond angle approaches $180^{\circ}$, however this is a strong deviation from the tetrahedral bond angle (as in $\mathrm{H}_{2} \mathrm{O}$ ) one might expect from simple molecular orbital arguments. Finally, both have a maximum near $150^{\circ}$, which is quite reasonable since most of the crystalline $\mathrm{SiO}_{2}$ polymorphs have bridging bond angles between $140^{\circ}$ and $155^{\circ}$. The bond angle distribution of Mozzi and Warren is much broader, however, which may be attributed to the inherent difficulties (and inaccuracies) in fitting the three pair correlation functions needed to analyze the X-ray scattering results with arbitrary functions. Additionally, the lack of good high angle scattering data may effectively truncate the results and lead to artificial broadening of the bond-angle distribution.

\section{Tetrasilicates $\left(\mathrm{K}_{2} \mathrm{Si}_{4} \mathrm{O}_{9}\right.$ and $\left.\mathrm{KMg}_{.5} \mathrm{Si}_{4} \mathrm{O}_{9}\right)$}

The second class of silicate glasses we have evaluated are tetrasilicates. These all have a total of +2 cationic charge balancing an $\mathrm{Si}_{4} \mathrm{O}_{9}-2$ cluster. The actual structures of both the crystalline and glassy compounds are much more complex. In the crystalline compounds, the silicates form long double stranded chains separated by cations. In the glass, these chains remain (as evidenced by the ${ }^{29} \mathrm{Si} \mathrm{NMR}$ ) however they are no longer ordered. In our study of these materials ${ }^{46}$ we hoped to both determine the Si-O-Si bond angle distributions to compare to the distribution from $\mathrm{SiO}_{2}$. Additionally, we attempted to evaluate the local ordering of the cations in the glass, similar to the ordering described by Gaskell in calcium modified silicate glasses ${ }^{171,172}$.

The experimental DAS spectra are shown in figure 8.12 for both $\mathrm{K}_{2} \mathrm{Si}_{4} \mathrm{O}_{9}$ and $\mathrm{KMg}_{.5} \mathrm{Si}_{4} \mathrm{O}_{9}$ glasses. Both spectra were taken at $9.4 \mathrm{~T}$ where the separation of the bridging and non-bridging oxygen peaks was the greatest. Spectra at $11.7 \mathrm{~T}$ were very similar and are not shown here. 

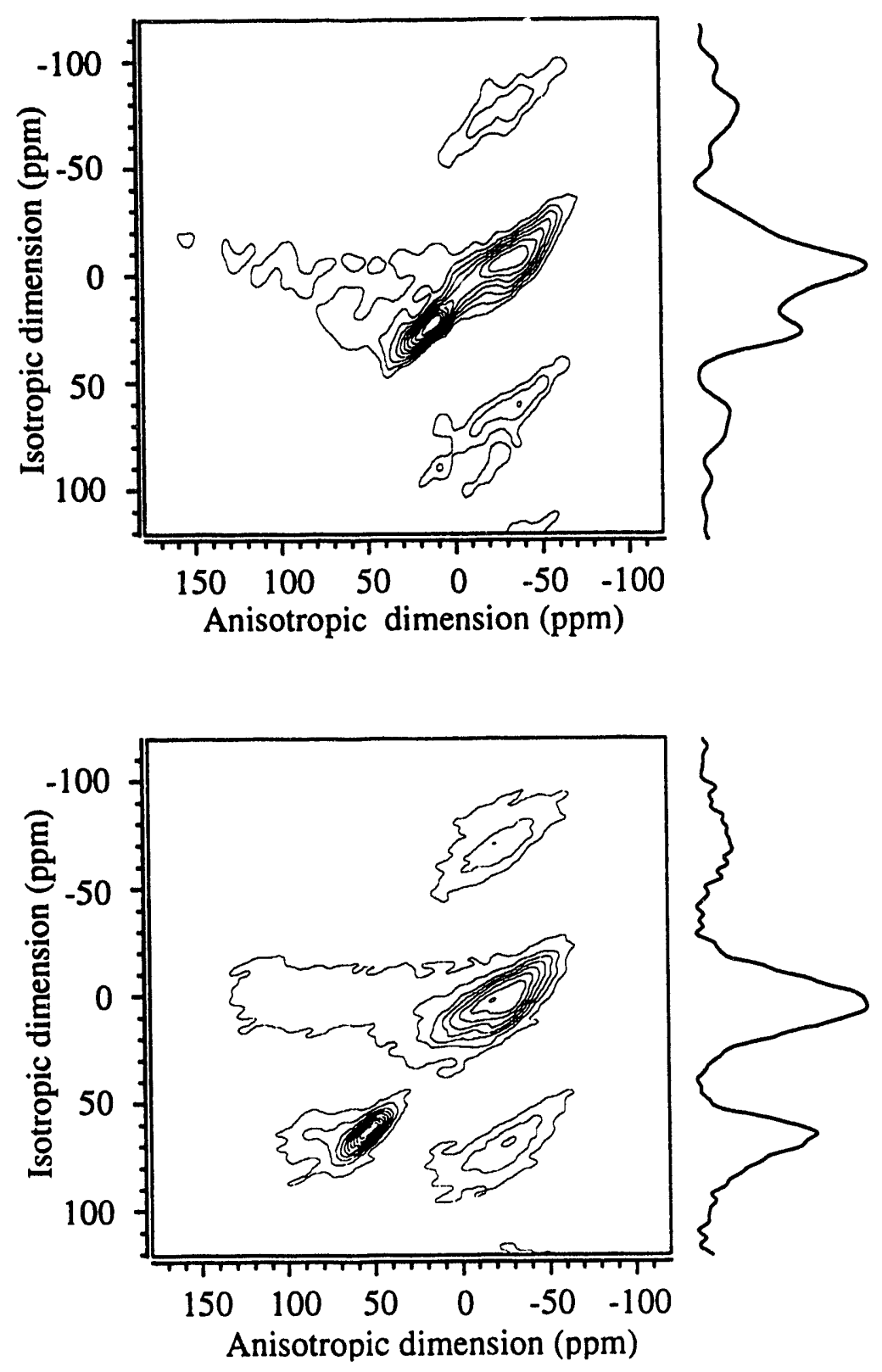

Figure 8.12 2D DAS Spectra of Tetrasilicate Glasses. The DAS spectrum on the left is for $\mathrm{K}_{2} \mathrm{Si}_{4} \mathrm{O}_{9}$ and the spectrum on the right is for $\mathrm{KMg}_{.5} \mathrm{Si}_{4} \mathrm{O}_{9}$. These spectra were taken at $9.4 \mathrm{~T}$ with the usual pulse sequences and acquisition parameters.

The isotropic bridging oxygen peak occurs at $0 \mathrm{ppm}$ in both spectra while the non-bridging oxygen peak occurs at $65 \mathrm{ppm}$ in the $\mathrm{K}_{2} \mathrm{Si}_{4} \mathrm{O}_{9}$ glass and $25 \mathrm{ppm}$ in the $\mathrm{KMg}_{.5} \mathrm{Si}_{4} \mathrm{O}_{9}$ glass. The two peaks on either side of the bridging oxygen correspond to spinning sidebands. The magnesium substituted glass has significantly worse signal-to-noise than the potassium tetrasilicate and cannot be used to extract a bond angle distribution. In the 
case of potassium tetrasilicate, we are able to simulate a number of the slices through the isotropic bridging oxygen site. The quadrupolar and chemical shift parameters from simulations of both the 9.4T and 11.7T DAS data sets are given in table 8.4. In both cases, only the peaks which were simulated are given. Intensities for extrapolated points were gotten from the one-dimensional DAS projection. In both cases the slice numbers are referenced to the 128 total points in the $\omega_{1}$ dimension following zero filling of the data in $t_{1}$. These parameters may then be converted into a bond angle distribution just as in the previous section. Both the 11.7T and 9.4T data give very similar distributions, as evidenced by the similarity of the quadrupolar and chemical shift parameters for the most intense slices ( 61 and 62 at $9.4 \mathrm{~T}$ and 69 and 60 at $11.7 \mathrm{~T}$ ). This resulting 9.4T bond angle distribution is shown in figure 8.13.

\begin{tabular}{ccccc||ccccc}
\hline Slice & $C_{Q}$ & $\eta_{Q}$ & Pop. & $\delta_{\text {iso,cs }}$ & Slice & $C_{Q}$ & $\eta_{Q}$ & Pop. & $\delta_{\text {iso,cs }}$ \\
\hline 56 & 5.73 & 0.09 & 0.21 & 57.3 & 54 & 6.12 & 0.10 & 0.51 & 57.7 \\
57 & 5.66 & 0.11 & 0.23 & 58.0 & 55 & 6.06 & 0.11 & 0.66 & 59.2 \\
58 & 5.65 & 0.12 & 0.26 & 60.5 & 56 & 5.90 & 0.12 & 0.83 & 58.4 \\
59 & 5.65 & 0.13 & 0.29 & 63.0 & 57 & 5.79 & 0.14 & 1.06 & 56.9 \\
60 & 5.55 & 0.16 & 0.31 & 62.9 & 58 & 5.72 & 0.16 & 1.13 & 60.3 \\
61 & 5.43 & 0.19 & 0.33 & 62.1 & 59 & 5.66 & 0.17 & 1.16 & 61.9 \\
62 & 5.33 & 0.20 & 0.33 & 61.7 & 60 & 5.56 & 0.19 & 1.15 & 62.7 \\
63 & 5.25 & 0.22 & 0.32 & 62.4 & 61 & 5.48 & 0.21 & 1.10 & 64.0 \\
64 & 5.28 & 0.23 & 0.30 & 66.2 & 62 & 5.40 & 0.22 & 0.97 & 65.5 \\
65 & 5.14 & 0.26 & 0.27 & 64.7 & 63 & 5.34 & 0.23 & 0.82 & 67.1 \\
66 & 5.10 & 0.28 & 0.25 & 67.3 & 64 & 5.28 & 0.24 & 0.66 & 68.8 \\
\hline
\end{tabular}

Table 8.4 $\mathrm{K}_{2} \mathrm{Si}_{4} \mathrm{O}_{9}$ Anisotropic Slice Fits from 9.4T and 11.7T DAS Spectrum. The simulations were performed using the computer programs in the appendix with the assumption that the chemical shift anisotropy was negligible, as in table 8.3. The parameters on the left correspond to $9.4 \mathrm{~T}$ data and on the right to $11.7 \mathrm{~T}$. 
Notice that the maximum in this distribution falls at about $140^{\circ}$, about $10^{\circ}$ less than in the $\mathrm{SiO}_{2}$ bond angle distribution. Also shown in figure 8.13 with a dashed line is the bond angle distribution calculated from a molecular dynamics simulation of $78 \mathrm{~K}_{2} \mathrm{O} \cdot 216 \mathrm{SiO}_{2} \cdot{ }^{177}$ It may be noted that this is significantly different in both the shape and maximum.

Returning to the DAS spectra in figure 8.12 , we note that the only major difference between the two spectra is in the position of the non-bridging oxygen peak. Both bridging site peaks are of approximately the same shape and width, as well the nonbridging peaks are of similar width.

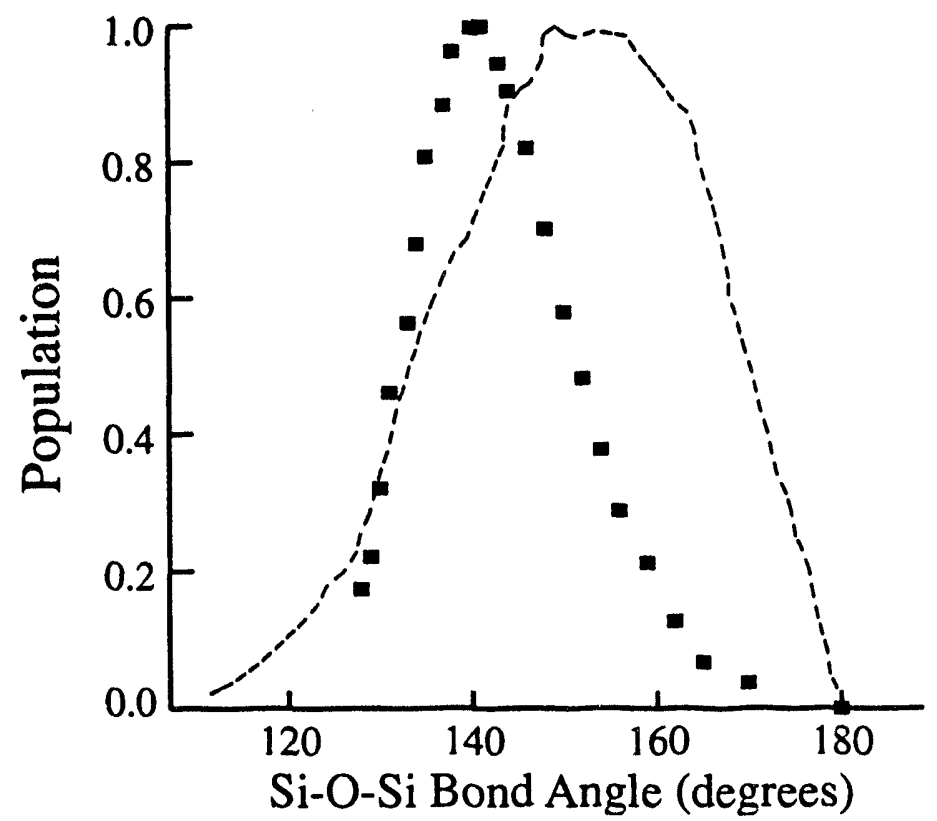

Figure 8.13 $\mathrm{K}_{2} \mathrm{Si}_{4} \mathrm{O}_{9}$ Bond Angle Distribution. The bond angle distribution for $\mathrm{K}_{2} \mathrm{Si}_{4} \mathrm{O}_{9}$ (squares) as well as a molecular dynamics simulation (dashed line) result are shown.

It is important to see that there is no sign of a non-bridging peak at $65 \mathrm{ppm}$ in the potassium magnesium tetrasilicate glass. This indicates that in the mixed cation glass there are no regions which are potassium "rich" and no region conversely which are magnesium "rich". In fact, the cation distribution must be anything but random in this glass, otherwise the peak at $25 \mathrm{ppm}$ would be a broad lump from 65 to $25 \mathrm{ppm}$. In the case of these glasses, for a $\mathrm{K}: \mathrm{Mg}$ ratio of $2: 1$ in the potassium magnesium tetrasilicate, 
the only way to produce an ordered $2: 1$ arrangement of $\mathrm{K}^{+}$and $\mathrm{Mg}^{+2}$ in the vicinity of the non-bridging oxygen atoms is to have an original coordination of four $\mathrm{K}^{+}$cations in the potassium tetrasilicate which then substitutes one $\mathrm{Mg}^{+2}$ for two $\mathrm{K}^{+}$cations in the potassium magnesium tetrasilicate. This will take the non-bridging oxygen atom from a coordination of 5 atoms ( 4 potassium atoms and 1 silicon) to 4 atoms ( 2 potassium atoms, 1 silicon and 1 magnesium). Since the magnesium is a much smaller cation of comparable size to a silicon cation, the magnesium substitution will produce a local nonbridging oxygen environment which is much more similar to a bridging oxygen environment than in the potassium tetrasilicate, hence the reduced isotropic chemical shift values. Additionally, since there will be a total of a +4 charge in the vicinity of every nonbridging oxygen atom, the non-bridging oxygen atoms themselves must be locally ordered and occur in distinct pairs. Additionally, both non-bridging oxygen atoms must not be coordinated to the same silicon atom, since this would necessitate the formation of $\mathrm{Q}^{2}$ (where $\mathrm{Q}^{n}$ stands for a silicon bonded to $n$ bridging oxygen atoms) species, which are not found in silicon NMR experiments. In fact all silicon atoms are in either $\mathrm{Q}^{3}$ or $\mathrm{Q}^{4}$ almost exclusively (50:50 ratio). ${ }^{178}$ This cationic ordering is in strong agreement with Gaskell et al. ${ }^{171,172}$ when they stated that the calcium cations were found to be in very ordered and regular arrangements in a tetrasilicate glass they studied. In fact, this study goes a step further to actually demonstrate absolute coordination in a modified tetrasilicate glass.

\section{Disilicates $\left(\mathrm{K}_{2} \mathrm{Si}_{2} \mathrm{O}_{5}\right)$}

The final class of glasses studied is the disilicates which consist of a mixture of cations totaling +2 charge and $\mathrm{Si}_{2} \mathrm{O}_{5}-2$ anion cluster. The crystalline form of these materials forms long chains which are separated by cations. The glasses also form the same chains, but again lacking the long range order of the crystal. Figure 8.14 shows the DAS spectrum of a potassium disilicate glass taken at $11.7 \mathrm{~T}$. This spectrum looks very 
much like the potassium tetrasilicate spectrum in figure 8.12. The main isotropic peak for the bridging oxygen sites occurs at $35 \mathrm{ppm}$ while the non-bridging oxygen peak occurs at $75 \mathrm{ppm}$. The overall width and position of these peaks are approximately the same as in the potassium tetrasilicate spectrum at 11.7T. The non-bridging peak indicates that, just as in the potassium tetrasilicate, the non-bridging oxygen atoms will be five-fold coordinated to one silicon and four potassium atoms. As in the previous two sections, the quadrupolar and chemical shift parameters were extracted with simulations of the bridging site slices. These are tabulated in the same form as before in table 8.5. As in the amorphous silica, the DAS spectrum was zero filled in the $t_{1}$ dimension to 256 points and the slices are referenced to these 256 points in the resulting $\omega_{1}$ dimension.

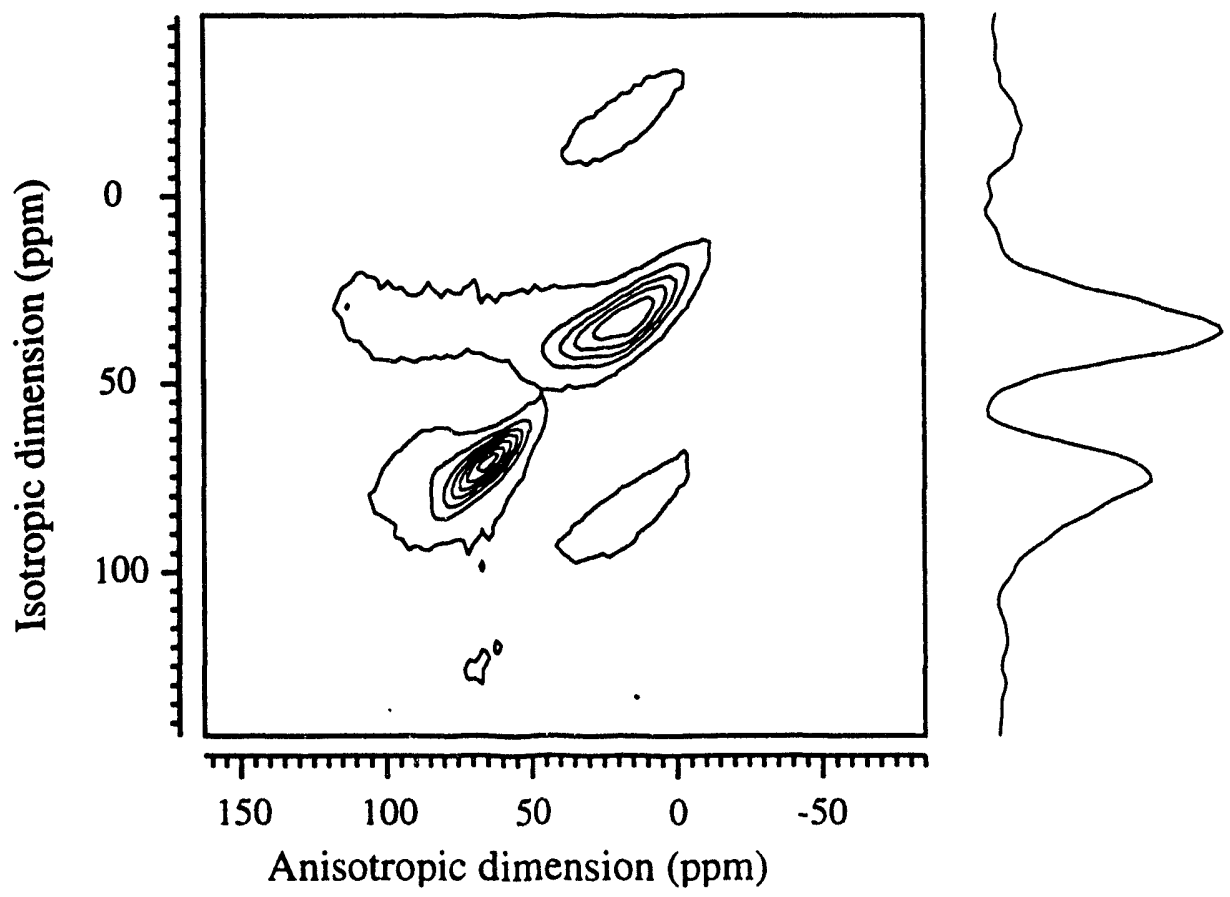

Figure 8.14 DAS Spectrum of $\mathrm{K}_{2} \mathrm{Si}_{2} \mathrm{O}_{5}$ glass at $11.7 \mathrm{~T}$. The DAS spectrum above was taken with the usual HyperSEDAS pulse sequence and parameters.

The first three slices $(110,111$ and 112$)$ seem to have unusually large isotropic chemical shifts relative to the expected trend from the other slices and thus they are attributed less significance (this is primarily due to the low signal-to-noise of these outer slices). These 
coupling constants are converted into a bond angle distribution in the usual fashion, which is shown below in figure 8.15.

\begin{tabular}{ccccc||ccccc}
\hline Slice & $C_{Q}$ & $\eta_{Q}$ & Pop. & $\delta_{\text {iso,cs }}$ & Slice & $C_{Q}$ & $\eta_{Q}$ & Pop. & $\delta_{\text {iso,cs }}$ \\
\hline 110 & 6.57 & 0.00 & 0.07 & 73.1 & 119 & 5.58 & 0.16 & 0.73 & 70.8 \\
111 & 6.52 & 0.00 & 0.11 & 73.7 & 120 & 5.48 & 0.18 & 0.77 & 71.2 \\
112 & 6.37 & 0.00 & 0.16 & 72.2 & 121 & 5.38 & 0.20 & 0.79 & 71.6 \\
113 & 6.08 & 0.01 & 0.23 & 67.7 & 122 & 5.30 & 0.21 & 0.78 & 72.5 \\
114 & 6.01 & 0.04 & 0.31 & 68.5 & 123 & 5.24 & 0.23 & 0.72 & 73.8 \\
115 & 5.94 & 0.05 & 0.40 & 69.2 & 124 & 5.16 & 0.25 & 0.62 & 74.5 \\
116 & 5.81 & 0.09 & 0.49 & 70.9 & 125 & 4.97 & 0.27 & 0.49 & 73.3 \\
117 & 5.72 & 0.12 & 0.58 & 68.1 & 126 & 4.84 & 0.28 & 0.35 & 73.2 \\
118 & 5.65 & 0.13 & 0.66 & 69.9 & 127 & 4.78 & 0.29 & 0.24 & 74.3 \\
\hline
\end{tabular}

Table $8.5 \mathrm{~K}_{2} \mathrm{Si}_{2} \mathrm{O}_{5}$ Anisotropic Slice Fits. The simulations were performed using the computer programs in the appendix with the assumption that the chemical shift anisotropy was negligible, as in table 8.3 and 8.4 .

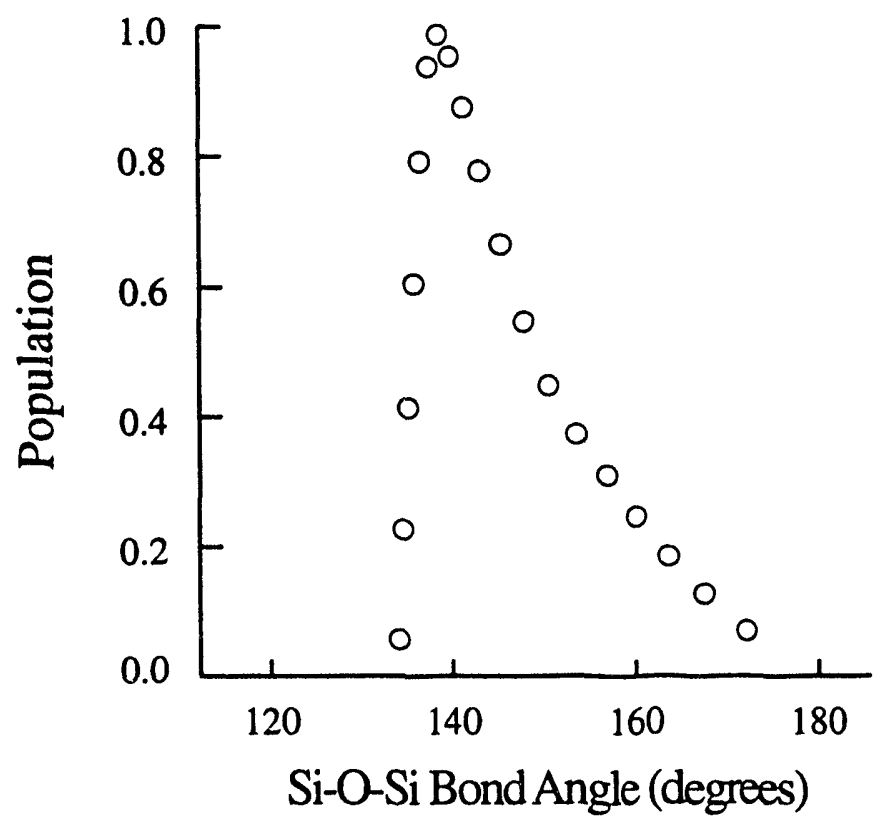

Figure 8.15 $\mathrm{K}_{2} \mathrm{Si}_{2} \mathrm{O}_{5}$ Bond Angle Distribution. The circles indicate the bond angle distribution extracted from the quadrupolar coupling constants in table 8.5. 
This bond angle distribution, just like the potassium tetrasilicate, has a maximum at approximate $140^{\circ}$ with error bars of $\pm 3^{\circ}$ in the peak region and $\pm 10^{\circ}$ in the wings. The usual sharp cut off at $130^{\circ}$ is observed, just as in the potassium tetrasilicate and in the silica. Any small variations between the tetrasilicate and disilicate bond angle distributions may be attributed to the random errors associated with the simulations. In disilicates such as $\mathrm{K}_{2} \mathrm{Si}_{2} \mathrm{O}_{5}$, it has been found as well previously that all silicon atoms are in $\mathrm{Q}^{3}$ coordination. ${ }^{178}$

\section{Conclusions}

The three bond angle distributions shown in the preceding sections are a good starting point to be able to understand the structures present in silicate glasses. The significance of these distributions is not well understood at this point, however some conclusions may be drawn. First, the bond angle maximum of the amorphous silica $\left(150^{\circ}\right)$ is significantly higher than the maximum for the polassium modified silicates $\left(140^{\circ}\right)$. This could possibly be attributed to the local ordering of the potassium cations around the nonbridging oxygen atoms. To achieve the cationic ordering observed earlier, it is necessary that the cations begin to form clusters early in the quenching of the glass and not be trapped in unfavorable environments as the glass viscosity increases. This is not difficult to envision, since the non-bridging oxygen sites are quite mobile due to the formation and breaking of bridging silicon-oxygen bonds as the cations migrate through the glass. As the glass forms, the cations must find energetic minima in the locally ordered clusters which controls the medium-range structure during the overall quenching of the glass. In the amorphous silica, there are no cations to lend mobility to oxygen atoms and therefore the glass transition temperature is much higher. Additionally as the silica fluid begins to quench into a glass, the lack of cations will cause a rapid loss of mobility and increase in the viscosity of the liquid. This might indicate that the silica bond angle distribution would be broader than the potassium silicate glasses were it not for the fact that the Si-O- 
Si potentials in the pure silica glass are significantly different than in the potassium silicate. Experimentally we observe that the silica and potassium silicate bond angle distributions are of similar width, indicating that the effect of the local ordering and lower quench temperature of the potassium silicates is comparable to the stronger potentials in the silica to control the overall bond distribution. It is difficult to attach any stronger conclusions at this time. Additional studies of the effect of quench rate and temperature on bond angle distributions, as well as compositional studies will be needed to give a complete picture of the processes occurring in glasses. 


\section{References}

\section{Bibliography}

(1) Baym, G. Lectures on Quantum Mechanics; The Benjamin/Cummings Publishing Company: Menlo Park, CA, 1969.

(2) Feynman, R. P.; Leighton, R. B.; Sands, M. The Feynman Lectures on Physics; Addison-Wesley Publishing Company: Reading, MA, 1965; Vol. 1-3.

(3) Sakurai, J. J. Advanced Quantum Mechanics; 10th ed.; Addison-Wesley Publishing Company, Inc.: Menlo Park, California, 1967.

(4) Sakurai, J. J. Modern Quantum Mechanics; Addison-Wesley Publishing Company, Inc.: Redwood City, CA, 1985.

(5) Schiff, L. I. Quantum Mechanics; 3rd ed.; McGraw-Hill, Inc.: New York, NY, 1968.

(6) Abragam, A. Principles of Nuclear Magnetism; Clarendon Press: Oxford, England, 1961.

(7) Ernst, R. R.; Bodenhausen, G.; Wokaun, A. Principles of Nuclear Magnetic Resonance in One and Two Dimensions; 1st ed.; Clarendon Press: Oxford, England, 1987; Vol. 14.

(8) Farrar, T. C.; Becker, E. D. Pulse and Fourier Transform NMR: Introduction to Theory and Methods; 1st ed.; Academic Press, Inc.: New York, NY, 1971.

(9) Fukushima, E.; Roeder, S. B. W. Experimental Pulse NMR: A Nuts and Bolts Approach; 1st ed.; Addison-Wesley Publishing Company, Inc.: Reading, MA, 1981.

(10) Harris, R. K. Nuclear Magnetic Resonance Spectroscopy: A Physicochemical View; 1st ed.; Longman Scientific \& Technical: Essex, England, 1986.

(11) Mehring, M. Principles of High-Resolution NMR in Solids; 2nd ed.; SpringerVerlag: Berlin, Germany, 1976; Vol. 11.

(12) Slichter, C. P. Principles of Magnetic Resonance; 3rd ed.; Springer-Verlag: Berlin, Germany, 1990; Vol. 1.

(13) Das, T. P.; Hahn, E. L. Nuclear Quadrupole Resonance Spectroscopy; Academic Press: New York, NY, 1958.

(14) Chmelka, B. F. Ph.D. Thesis, University of California, Berkeley, 1989. 
(15) Mueller, K. T. Ph.D. Thesis, University of California, Berkeley. 1991.

(16) Sun, B. Q. Ph.D. Thesis, University of California, Berkeley, 1992.

(17) Pose, M. E. Elementary Theory of Angular Momentum; John Wiley \& Sons: New iork, NY, 1957.

(18) Edmonds, A. R. Angular Momentum in Quantum Mechanics; Princeton University Press: Princeton, NJ, 1960.

(19) Zare, R. N. Angular Momentum: Understanding Spatial Aspects in Chemistry and Physics; John Wiley \& Sons: New York, NY, 1988.

(20) Dyson, F. Phys. Rev. 1949, 75, 486.

(21) Dyson, F. Phys. Rev. 1949, 75, 1736.

(22) Tossell, J. A. Phys. Chem. Min. 1984, 10, 137.

(23) Casimir, H. B. G. On the Interaction Between Atomic Nuclei and Electrons; Teyler's Tweede Genootschap: Haarlem, 1936.

(24) Cohen, M. H.; Reif, F. Sol. State Phys. 1957, 5, 321.

(25) Vega, S.; Pines, A. J. Chem. Phys. 1977, 66, 5624-5644.

(26) Vega, S. J. Chem. Phys. 1978, 68, 5518-5527.

(27) Vega, S.; Naor, Y. J. Chem. Phys. 1981, 75, 75-86.

(28) Vega, S. Physical Review A 1981, 23, 3152-3172.

(29) Man, P. P. J. Magn. Reson. 1986, 67, 78-90.

(30) Man, P. P.; Theveneau, H.; Papon, P. J. Magn. Reson. 1985, 64, $271-277$.

(31) Man, P. P. J. Magn. Reson. 1988, 77, 148-154.

(32) Man, P. P.; Klinowski, J.; Trokiner, A.; Zanni, H.; Papon, P. Chem. Phys. Lett. 1988, 151, 143-150.

(33) Man, P. P. J. Magn. Reson. 1991, 94, 258-267.

(34) Man, P. P. Mol. Phys. 1993, 78, 307-318.

(35) Wokaun, A.; Ernst, R. R. J. Chem. Phys. 1977, 67, 1752-1758.

(36) Bain, A. D. J. Magn. Reson. 1984, 56, 418-427. 
(37) Bodenhausen, G.; Kogler, H.; Ernst, R. R. J. Magn. Reson. 1984, 58, 370-388.

(38) Haeberlen, U. High Resolution NMR in Solids - Selective Averaging; Academic Press: New York, NY, 1976.

(39) Carr, H. Y.; Purcell, E. M. Phys. Rev. 1954, 94, 630.

(40) Gill, D.; Meiboom, S. Rev. Sci. Instrum. 1958, 29, 688.

(41) Alderman, D. W.; Solum, M. S.; Grant, D. M. J. Chem. Phys. 1986, 84, 37173725.

(42) Mueller, K. T.; Sun, B. Q.; Chingas, G. C.; Zwanziger, J. W.; Terao, T.; Pines, A. J. Magn. Reson. 1990, 86, 470-487.

(43) Llor, A.; Virlet, J. Chem. Phys. Lett. 1988, 152, 248-253.

(44) Eastman, M. A.; Grandinetti, P. J.; Lee, Y. K.; Pines, A. J. Magn. Reson. 1992 , $98,333$.

(45) Mueller, K. T.; Wu, Y.; Chmelka, B. F.; Stebbins, J.; Pines, A. J. Am. Chem. Soc. 1991, 113, 32-38.

(46) Farnan, I.; Grandinetti, P. J.; Baltisberger, J. H.; Stebbins, J. F.; Werner, U.; Eastman, M. A.; Pines, A. Nature 1992, 358, 31-35.

(47) Chmelka, B. F.; Mueller, K. T.; Pines, A.; Stebbins, J.; Wu, Y.; Zwanziger, J. W. Nature 1989, 339, 42-43.

(48) Mueller, K. T.; Baltisberger, J. H.; Wooten, E. W.; Pines, A. J. Am. Chem. Soc. 1992, 96, 7001-7004.

(49) Sun, B. Q.; Baltisberger, J. H.; Wu, Y.; Samoson, A.; Pines, A. Solid State NMR 1992, 1, 26i-295.

(50) Mueller, K. T.; Wooten, E. W.; Pines, A. J. Magn. Reson. 1991, 92, 620-627.

(51) Mueller, K. T.; Chingas, G. C.; Pines, A. Rev. Sci. Instrum. 1991, 62, 1445-1452.

(52) Grandinetti, P. J.; Lee, Y. K.; Baltisberger, J. H.; Sun, B. Q.; Pines, A. J. Magn. Reson. A 1993, 102, 195-204.

(53) Grandinetti, P. J.; Baltisberger, J. H.; Llor, A.; Lee, Y. K.; Werner, U.; Eastman, M. A.; Pines, A. J. Magn. Reson. A 1993, 103, 72-81.

(54) Baltisberger, J. H.; Gann, S. L.; Wooten, E. W.; Chang, T. H.; Mueller, K. T.; Pines, A. J. Am. Chem. Soc. 1992, 114, 7489-4793. 
(55) Samoson, A.; Lippmaa, E.; Pines, A. Mol. Phys. 1988, 65, 1013-1018.

(56) Gann, S. L.; Baltisberger, J. H.; Pines, A. Chem. Phys. Lett. 1993, in press,

(57) Elbaum, N. C.; Haw, J. F. J. Magn. Reson. 1991, 91, 199-203.

(58) Maricq, M. M.; Waugh, J. S. J. Chem. Phys. 1979, 70, 3300-3316.

(59) Herzfeld, J.; Berger, A. E. J. Chem. Phys. 1980, 73, 6021-6030.

(60) Jakobsen, H. J.; Daugaard, P.; Langer, V. J. Magn. Reson. 1988, 76, 162-168.

(61) Jakobsen, H. J.; Skibsted, J.; Bildsøe, H.; Nielsen, N. C. J. Magn. Reson. 1989, $85,173-180$.

(62) Samoson, A.; Kundla, E.; Lippmaa, E. J. Magn. Reson. 1982, 49, 350-357.

(63) Samoson, A. Chem. Phys. Lett. 1985, 119, 29-32.

(64) Amoureux, J. P.; Fernandez, C.; Lefebvre, F. Magnetic Resonance in Chemistry $1990,28,5-10$.

(65) Dec, S. F.; Maciel, G. E. J. Magn. Reson. 1990, 87, 153-159.

(66) Dec, S. F.; Maciel, G. E.; Fitzgerald, J. J. J. Am. Chem. Soc. 1990, 112, 90699077.

(67) Jäger, C.; Dupree, R.; Kohn, S. C.; Mortuza, M. G. J. Non-Cryst. Solids 1993, in press.

(68) Jäger, C. J. Magn. Reson. 1992, 99, 353-362.

(69) Lefebvre, F.; Amoureux, J. P.; Fernandez, C.; Derouane, E. G. J. Chem. Phys. 1987, 86, 6070-6076.

(70) Massiot, D.; Kahn-Harari, A.; Michel, D.; Muller, D.; Taulelle, F. Magnetic Resonance in Chemistry 1990, 28, S82-88.

(71) Massiot, D.; Bessada, C.; Coutures, J. P.; Taulelle, F. J. Magn. Reson. 1990, 90, 231-242.

(72) Oldfield, E.; Kirkpatrick, R. J. Science 1985, 227, 1537-1544.

(73) Oldfield, E.; Kyung, H.; Timken, C.; Montez, B.; Ramachandran, R. Nature 1985, 318, 163-165.

(74) Skibsted, J.; Nielsen, N. C.; Bildsøe, H.; Jakobsen, H. J. J. Magn. Reson. 1991, 95, 88-117. 
(75) Skibsted, J.; Bildsøe, H.; Jakobsen, H. J. J. Magn. Reson. 1991, 92, 669-676.

(76) Zheng, Z.; Gan, Z.; Sethi, N. K.; Alderman, D. W.; Grant, D. M. J. Magn. Reson. 1991, 95, 509-522.

(77) Bodenhausen, G.; Kempsell, S. P.; Freeman, R.; Hill, H. D. W. J. Magn. Reson. 1979, 35, 337-342.

(78) Ganapathy, S.; Shore, J.; Oldfield, E. Chem. Phys. Lett. 1990, 169, 301-306.

(79) Levitt, M. H. J. Magn. Reson. 1989, 82, 427-433.

(80) Sardashti, M.; Maciel, G. E. J. Magn. Reson. 1987, 72, 467-474.

(81) Sethi, N. K.; Alderman, D. W.; Grant, D. M. Mol. Phys. 1990, 71, 217-238.

(82) Nagayama, K.; Bachmann, P.; Wüthrich, K.; Ernst, R. R. J. Magn. Reson. 1978, $31,133$.

(83) Nagayama, K.; Kumar, A.; Wüthrich, K.; Ernst, R. R. J. Magn. Reson. 1980, 40, 321.

(84) Bax, A.; Griffey, R. H.; Hawkins, B. L. J. Magn. Reson. 1983, 55, 301.

(85) Wider, G.; Macura, S.; Kumar, A.; Ernst, R. R.; Wüthrich, K. J. Magn. Reson. 1984, 56, 207.

(86) Kolbert, A. C.; Levitt, M. H.; Griffin, R. G. J. Magn. Reson. 1989, 85, 42.

(87) Van Vleck, J. H. Phys. Rev. 1948, 74, 1168.

(88) States, D. J.; Haberkorn, R. A.; Ruben, D. J. J. Magn. Reson. 1982, 48, 286-292.

(89) Marion, D.; Wüthrich, K. Biochem. Biophys. Res. Commun. 1983, 113, 967.

(90) Drobny, G.; Pines, A.; Sinton, S.; Weitekamp, D.; Wemmer, D. Symp. Faraday Soc. 1979, 13, 49.

(91) Bax, A.; Mehlkopf, A. F.; Smidt, J. J. Magn. Reson. 1979, 35, 373-377.

(92) Hartmann, S. R.; Hahn, E. L. Phys. Rev. 1962, 128, $2042-2053$.

(93) Pines, A.; Gibby, M. G.; Waugh, J. S. J. Chem. Phys. 1973, 59, 569-590.

(94) Stejskal, E. O.; Schaefer, J.; Waugh, J. S. J. Magn. Reson. 1977, 28, 105-112.

(95) Nielsen, N. C.; Sørensen, O. W. J. Magn. Reson. 1992, 99, 214-222.

(96) Vega, A. J. J. Magn. Reson. 1992, 96, 50-68. 
(97) Walter, T. H.; Turner, G. L.; Oldfield, E. J. Magn. Reson. 1988, 76, 106-120.

(98) Harris, R. K.; Nesbitt, G. J. Magn. Reson. 1988, 78, 245-256.

(99) Haase, J.; Pfeifer, H. J. Magn. Reson. 1990, 86, 217-226.

(100) Wu, Y.; Lewis, D.; Frye, J. S.; Palmer, A. R.; Wind, R. A. J. Magn. Reson. 1992, $100,425-430$.

(101) Power, W. P.; Wasylishen, R. E.; Mooibroek, S.; Pettitt, B. A.; Danchura, W. J. Phys. Chem. 1990, 94, 591-598.

(102) Merwin, L. H.; Sebald, A. Solid State NMR 1992, 1, 45-47.

(103) Edwards, J. C.; Ellis, P. D. Magnetic Resonance in Chemistry 1990, 28, S59-67.

(104) Walter, T. H.; Oldfield, E. J. Phys. Chem. 1989, 93, 6744-6751.

(105) Vega, A. J. Solid State NMR 1992, 1, 17-32.

(106) Hayashi, S.; Hayamizu, K. Chem. Phys. Lett. 1993, 203, 319-324.

(107) Doty, F. D.; Ellis, P. D. Rev. Sci. Instrum. 1981, 52, 1868-1875.

(108) Samoson, A.; Lippmaa, E. J. Magn. Reson. 1989, 84, 410-416.

(109) Bax, A.; Szeverenyi, N. M.; Maciel, G. E. J. Magn. Reson. 1983, 52, 147-152.

(110) Szeverenyi, N. M.; Bax, A.; Maciel, G. E. J. Magn. Reson. 1985, 61, 440-447.

(111) Gan, Z. J. Am. Chem. Soc. 1992, 114, 8307-8309.

(112) Wu, Y.; Sun, B. Q.; Pines, A. J. Magn. Reson. 1990, 89, 297-309.

(113) Samoson, A.; Sun, B. Q.; Pines, A. In Pulsed Magnetic Resonance: NMR, ESR and Optics - A Recognition of E.L. Hahn; D. M. S. Bagguley, Ed.; Clarendon Press: Oxford, 1992; pp 80-94.

(114) Wu, Y.; Chmelka, B. F.; Pines, A.; Davis, M. E.; Grobet, P. J.; Jacobs, P. A. Nature 1990, 346, 550-552.

(115) Dixon, W. T.; Schaefer, J.; Sefcik, M. D.; Stejskal, E. O.; McKay, R. A. J. Magn. Reson. 1982, 49, 341-345.

(116) Dixon, W. T. J. Magn. Reson. 1985, 64, 332-333.

(117) Kolbert, A. C.; Griffin, R. G. Chem. Phys. Lett. 1990, 166, 87-91.

(118) Geen, H.; Bodenhausen, G. J. Am. Chem. Soc. 1993, 115, 1579-1580. 
(119) Geen, H.; Bodenhausen, G. J. Chem. Phys. 1992, 97, 2928-2937.

(120) Raleigh, D. P.; Olejniczak, E. T.; Griffin, R. G. J. Magn. Reson. 1991, 93, 472484.

(121) Raleigh, D. P.; Kolbert, A. C.; Griffin, R. G. J. Magn. Reson. 1990, 89, 1-9.

(122) Raleigh, D. P.; Olejniczak, E. T.; Vega, S.; Griffin, R. G. J. Magn. Reson. 1987, 72, 238-250.

(123) Alderman, D., MAH with $\pi$ pulses.

(124) Hikita, T.; Aika, K. I.; Onishi, T. Cat. Lett. 1990, 4, 157-162.

(125) Aika, K.; Fujimoto, N.; Kobayashi, M.; Iwamatsu, E. J. Cat. 1991, 127, 1-8.

(126) Bansal, N. P.; Doremus, R. H. Handbook of Glass Properties; Academic Press: Orlando, FL, 1986.

(127) Rosseinsky, M. J.; Ramirez, A. P.; Glarum, S. H.; Murphy, D. W.; Haddon, R. C.; Hebasrd, A. F.; Palstra, T. T. M.; Kortan, A. R.; Zahurak, S. M.; Makhija, A. V. Phys. Rev. Lett. 1991, 66, 2830-2832.

(128) Cheng, J. T.; Edwards, J. C.; Ellis, P. D. J. Phys. Chem. 1990, 94, 553-561.

(129) Cheng, J. T.; Ellis, P. D. J. Phys. Chem. 1989, 93, 2549-2555.

(130) Deshpande, V. T.; Sirdeshmukh, D. B. Acta Cryst. 1961, 14, 353-355.

(131) Braekken, H.; Harang, L. Z. Krist. 1930, 75, 538-549.

(132) Smith, H. W., Jr.; Colby, M. Y. Z. Krist. 1940, 103, 90-95.

(133) Nord, A. G. Acta Cryst. 1974, B30, 1640-1641.

(134) Shamsuzzoha, M.; Lucas, B. W. Acta Cryst. 1982, B38, 2353-2357.

(135) Dean, C.; Hambley, T. W.; Snow, M. R. Acta Cryst. 1984, C40, 1512-1515.

(136) Segel, S. L. J. Chem. Phys. 1980, 73, 4146-4147.

(137) Pan, Y.; Gullion, T.; Schaefer, J. J. Magn. Reson. 1990, 90, 330-340.

(138) Schmidt, A.; McKay, R. A.; Schaefer, J. J. Magn. Reson. 1992, 96, 644-650.

(139) Doty, F. D.; Inners, R. R.; Ellis, P. D. J. Magn. Reson. 1981, 43, 399-416.

(140) Doty, F. D.; Connick, T. J.; Ni, X. Z.; Clingan, M. N. J. Magn. Reson. 1988, 77, 536-549. 
(141) Simpson, H. J., Jr.; Marsh, R. E. Acta Cryst. 1966, 20, 550-555.

(142) Tossell, J. A.; Lazzeretti, P. Phys. Chem. Min. 1988, 15, 564-569.

(143) Coretsopoulos, C.; Lee, H. C.; Ramli, E.; Reven, L.; Rauchfuss, T. B.; Oldfield, E. Phys. Rev. B 1989, 39, 781-784.

(144) Ganapathy, S.; Schramm, S.; Oldfield, E. J. Chem. Phys. 1982, 77, 4360-4365.

(145) Lee, H. C.; Cummings, K.; Hall, K.; Hager, L. P.; Oldfield, E. J. Bio. Chem. 1988, $263,16118-16124$.

(146) Oldfield, E.; Kinsey, R. A.; Smith, K. A.; Nichols, J. A.; Kirkpatrick, R. J. J. Magn. Reson. 1983, 51, 323-329.

(147) Schramm, S.; Oldfield, E. J. Am. Chem. Soc. 1984, 106, 2502-2506.

(148) Thompson, A. R.; Kunwar, A. C.; Gutowsky, H. S.; Oldfield, E. J. Chem. Soc. Delton Trans. 1987, 2317-2322.

(149) Turner, G. L.; Chung, S. E.; Oldfield, E. J. Magn. Reson. 1986, 64, 316-324.

(150) Timken, H. K. C.; Schramm, S. E.; Kirkpatrick, R. J.; Oldfield, E. J. Phys. Chem. 1987, 91, 1054-1058.

(151) Yang, S.; Shore, J.; Oldfield, E. J. Magn. Reson. 1992, 99, 408-412.

(152) Warren, B.; Bragg, W. L. Z. Kristallogr. 1928, 69, 168-193.

(153) Toger, F. J. Z. Kristallogr. 1968, 127, 291-308.

(154) Wyckoff, R. W. G. Crystal Structures; Interscience: New York, NY, 1948; Vol. 3.

(155) Morimoto, N.; Appleman, D. E.; Evans, H. T., Jr. Z. Kristallogr. 1960, 114, 120147.

(156) Midgely, C. M. Acta Crystallogr. 1952, 5, 307-312.

(157) Press, W. H.; Flannery, B. P.; Teukolsky, S. A.; Vetterling, W. T. Numerical Recipes. The Art of Scientific Computing; 1st ed.; Cambridge University Press: Cambridge, England, 1986.

(158) Kintzinger, J. P. NMR: Basic Principles and Progress; Springer-Verlag: Berlin, Germany, 1978.

(159) Kirkpatrick, R. J. Rev. Minerol. 1988, 18, 341-403.

(160) Elliott, S. R. Physics of Amorphous Materials; 2nd ed.; Longman Scientific \& Technical: Essex, England, 1990. 
(161) Mozzi, R. L.; Warren, B. E. Journal of Applied Crystalography 1969, 2, 164.

(162) Harris, I. A., Jr.; Bray, P. J. Phys. Chem. Glasses 1980, 21, 156-159.

(163) Galeener, F. L. Phil. Mag. B 1985, 51, L1-L6.

(164) Devine, R. A. B.; Dupree, R.; Farnan, I.; Capponi, J. J. Phys. Rev. B 1987, 35, 2560-2562.

(165) Dupree, R.; Holland, D.; McMillan, P. W.; Pettifer, R. F. J. Non-Cryst. Solids $1984,68,399-410$.

(166) Dupree, R.; Pettifer, R. F. Nature 1984, 308, 523-525.

(167) Pettifer, R. F.; Dupree, R.; Farnan, I.; Sternberg, U. J. Non-Cryst. Solids 1988, 106, 408.

(168) Elliot, S. R. Science 1992, 357, 650.

(169) Glass Science and Technology; Greaves, G. N., Ed.; 1990; Vol. 4B, pp 1-76.

(170) Greaves, G. N.; Fontaine, A.; Lagarde, P.; Raoux, D.; Gurman, S. J. Nature 1981, 293, 611-616.

(171) Eckersley, M. C.; Gaskell, P. H.; Barnes, A. C.; Chieux, P. Nature 1988, 335, 525-527.

(172) Gaskell, P. H.; Eckersley, M. C.; Barnes, A. C.; Chieux, P. Nature 1991, 350, 675-677.

(173) Stebbins, J. F. J. Non-Cryst. Solids 1988, 106, 359-369.

(174) Geissberger, A. E.; Bray, P. J. J. Non-Cryst. Solids 1983, 54, 121-137.

(175) Spearing, D. R.; Farnan, I.; Stebbins, J. F. Phys. Chem. Min. 1993, in press.

(176) Grandinetti, P. J.; Baltisberger, J. H.; Farnan, I.; Werner, U.; Pines, A.; Stebbins, J. F. in preparation 1993,

(177) Huang, C.; Cormack, A. N. J. Chem. Phys. 1991, 95, 3634-3642.

(178) Eckert, H. Progress in Magnetic Resonance Spectroscopy 1992, 24, 159-293.

\section{Appendices}

Computer programs 
A variety of computer programs were developed which were used to simulate the one dimensional VAS spectra or slices from two-dimensional DAS spectra. The first program, CQP, outputs a file which contains frequency and intensity pairs over the full sweep width desired. The second program, MINUITCQ, uses the MINUIT (CERN) library of minimization routines to iteratively arrive at a least-squares best fit to an input data set. The first program is useful for rapidly getting the initial parameters in a fit in the correct ranges and for exploring the effects of small deviations in parameters. The second program uses both simplex and gradient minimization techniques and provides an error matrix which may be used to evaluate the overall errors in each of the fit parameters.

\section{CQP - VAS Spectral Simulation Program}

\begin{tabular}{|c|c|}
\hline C & PROGRAM CQP \\
\hline C & CALCULATES FREQUENCIES FOR EACH CRYSTALLITE ORIENTATION \\
\hline C & ASSUMING THE PRESENCE OF BOTH SECOND-ORDER QUADRUPOLAR \\
\hline $\mathrm{C}$ & INTERACTIONS AS WELL AS CHEMICAL SHIFT ANISOTROPY. THE PAS \\
\hline $\mathrm{C}$ & OF THESE TWO INTERACTIONS DO NOT NEED TO NECESSARILY COINCIDE \\
\hline C & AND IN FACT THE CSA PAS IS DESCRIBED FIRST REIJATIVE TO THE \\
\hline C & QUADRUPOLAR PAS AND THEN BOTH ARE ROTATED TO THE ROTOR FRAME \\
\hline C & AND THEN FINALLY TO THE LABORATORY FRAME. IT IS ASSUMED AS \\
\hline $\mathrm{C}$ & WELL THAT THE SPINNING SPEED IS FAST ENOUGHT TO ELIMINATE ALL \\
\hline $\mathrm{C}$ & TIME DEPENDANT TERMS IN THE FREQUENCY EXPRESSION. THE POWDER \\
\hline C & PATTERS ARE CALCULATED USING A METHOD \\
\hline C & \\
\hline C & This was written by Jay Baltisberger \\
\hline C & Chemistry Department \\
\hline C & Berea College \\
\hline C & Berea, KY 40404 \\
\hline C & \\
\hline C & while at the University of California, Berkeley in the \\
\hline $\mathrm{C}$ & laboratory of Prof. A. Pines \\
\hline C & \\
\hline C & THIS PROGRAM IS DESIGNED TO RUN ON STANDARD UNIX TYPE MACHINES \\
\hline C & THE FOLLOWING PROGRAMS AND SUBROUTINES NEED TO BE COMPILED AND \\
\hline C & LINKED. \\
\hline C & \\
\hline C & \\
\hline C & COMPILE LIST \\
\hline C & cqp.f \\
\hline C & $d r . f$ \\
\hline C & $\mathrm{dr} 2 . \mathrm{f}$ \\
\hline $\mathrm{C}$ & jran.f \\
\hline C. & $f f t 1 . f$ \\
\hline C & powdim8.f \\
\hline $\mathrm{C}$ & Iines.f \\
\hline
\end{tabular}




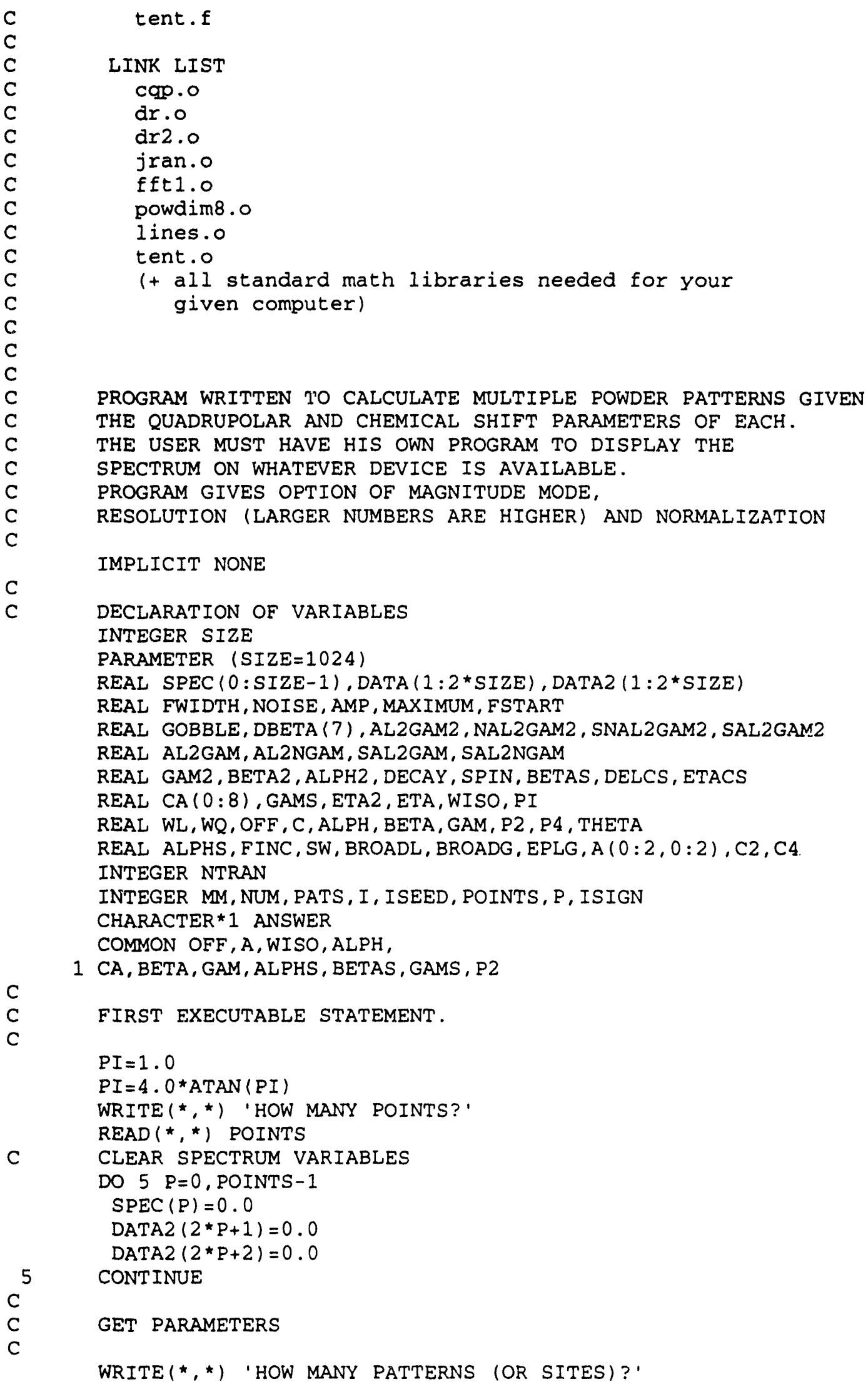




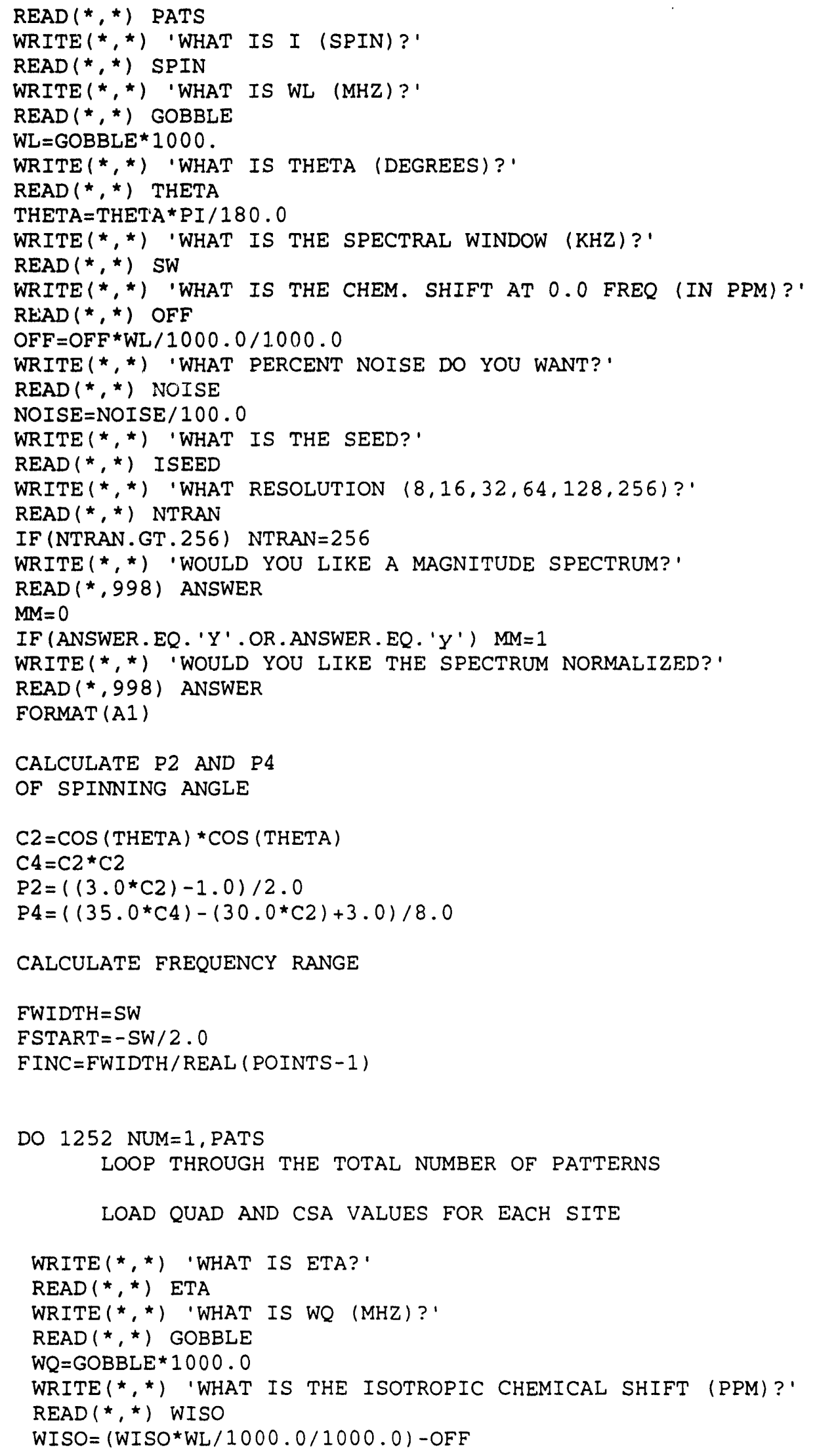




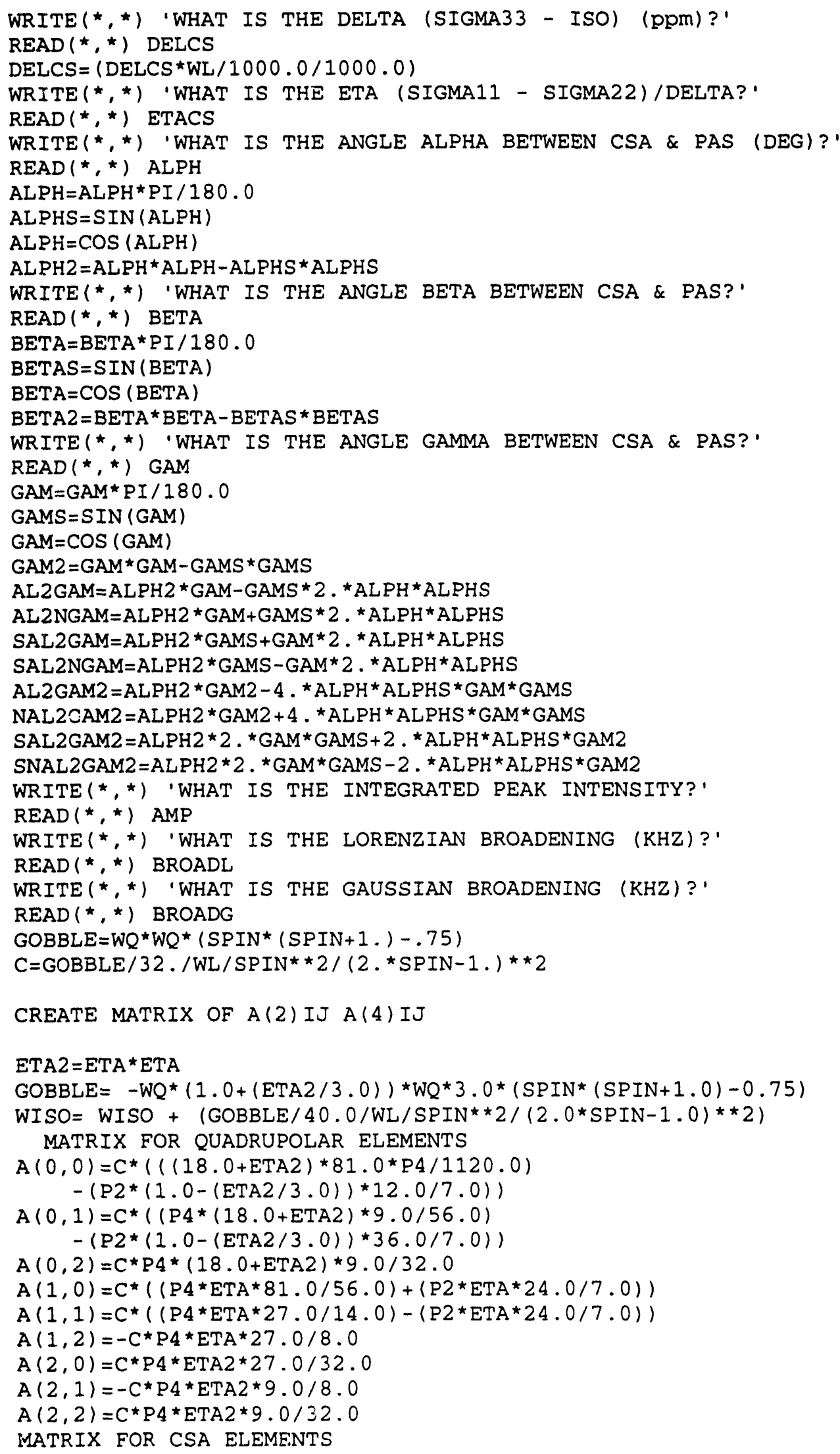




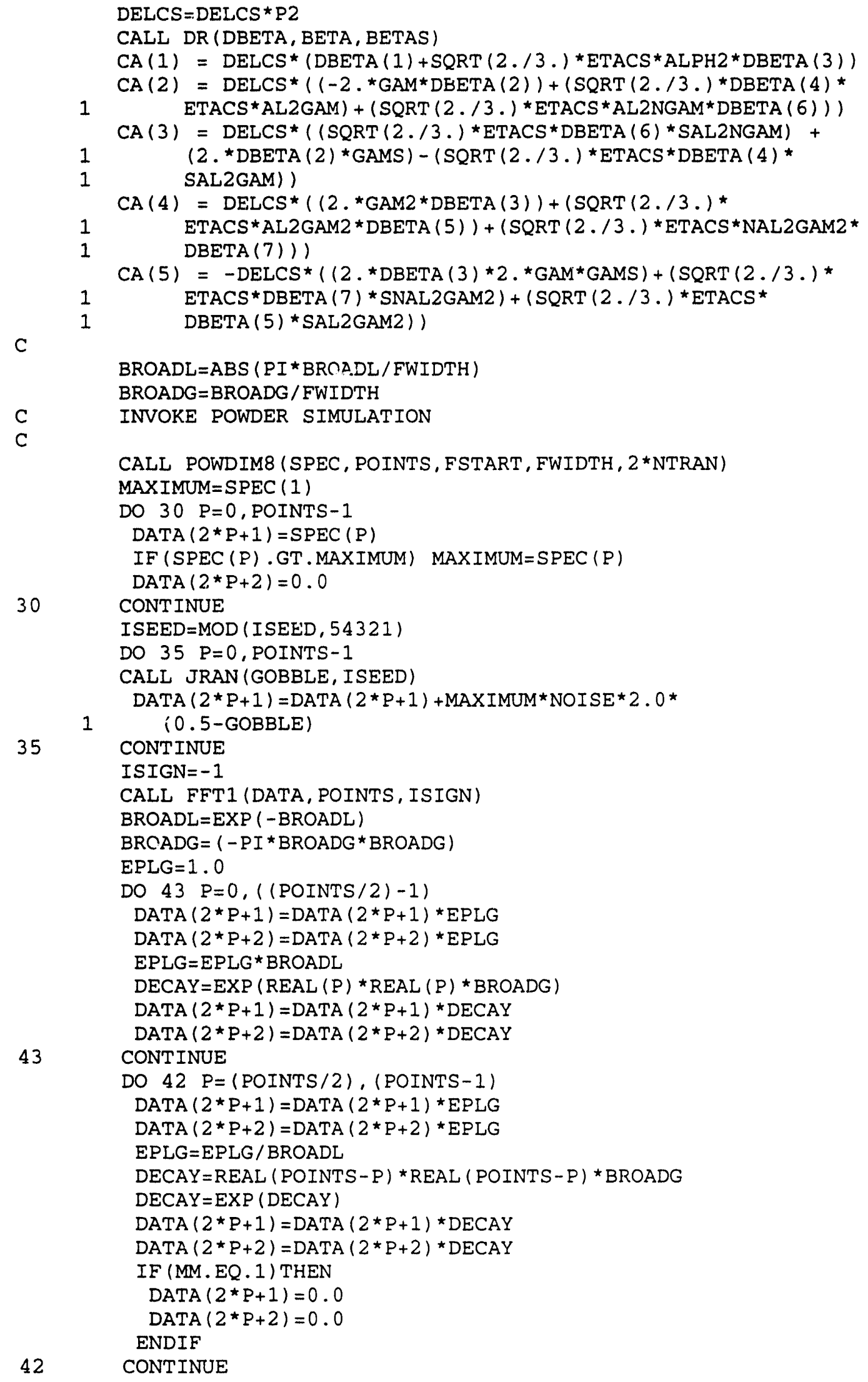


ISIGN $=1$

CALL FFT1 (DATA, POINTS, ISIGN)

C

FIND AREA OF SPECTRUM

MAXIMUM $=0.0$

DO $100 \quad P=0$, POINTS -1

100 MAXIMUM=MAXIMUM+DATA $(2 \star \mathrm{P}+1)$

C NORMALIZE SPECTRUM SO THAT LARGEST POINT IS UNITY

DO $201 \quad P=6$, POINTS-1

$\operatorname{SPEC}(P)=0.0$

$\operatorname{DATA} 2(2 \star \mathrm{P}+1)=\operatorname{DATA} 2(2 * \mathrm{P}+1)+($ AMP $* \operatorname{DATA}(2 * \mathrm{P}+1) / \mathrm{MAXIMUM})$

201

DATA2 $(2 \star P+2)=$ DATA2 $(2 * P+2)+($ AMP $*$ DATA $(2 \star P+2) /$ MAXIMUM $)$

1252

CONTINUE

CONTINUE

IF (MM.EQ.1) THEN

DO $8915 \mathrm{P}=0$, POINTS -1

$\operatorname{DATA2}(2 * \mathrm{P}+1)=\operatorname{SQRT}\left(\left(\operatorname{DATA} 2\left(2{ }^{\star} \mathrm{P}+1\right) * \operatorname{DATA} 2\left(2{ }^{\star} \mathrm{P}+1\right)\right)+\right.$

8915 CONTINUE $(\operatorname{DATA} 2(2 \star \mathrm{P}+2) * \operatorname{DATA} 2(2 \star \mathrm{P}+2)))$

ENDIF

IF (ANSWER.EQ.' $Y$ ') THEN

MAXIMUM $=0.0$

DO 234 I $=0$, POINTS -1

MAXIMUM $=$ MAXIMUM+DATA2 $(2 * I+1)$

234

CONTINUE

DO $235 I=0$, POINTS -1

$\operatorname{DATA} 2(2 \star I+1)=\operatorname{DATA} 2(2 \star I+1) /$ MAXIMUM

235 CONTINUE

ENDIF

C DISPLAY THE RESULT

OPEN (UNIT $=10$, FILE = ' POWD. DAT' , STATUS= ' UNKNOWN' ')

REWIND (10)

DO $2657 I=0$, POINTS - 1

$\operatorname{WRITE}(10, *) \quad(\operatorname{REAL}(I) * \operatorname{FINC}+\operatorname{FSTART}), \operatorname{DATA} 2(2 * I+1)$

2657 CONTINUE

CLOSE (10)

END

C

SUBROUTINE DR ( $D, C B, S B)$

C

IMPLICIT NONE

C

C DELCLARATION OF ARGUMENTS

REAL $D(7), C B, S B$

C

$D(1)=\left(\left(3 .{ }^{*} C B^{*} C B\right)-1.\right) / 2$.

$D(2)=-\operatorname{SQRT}(3 . / 2) *. S B^{\star} C B$

$D(3)=\operatorname{SQRT}(3 . / 8) * S B * S$.

$D(4)=-((1 .+C B) / 2) * S$.

$D(5)=((1 .+C B) / 2) *.((1 .+C B) / 2$.

$D(6)=((1 .-C B) / 2) * S$.

$D(7)=((1 .-C B) / 2) *.((1 .-C B) / 2$.

RETURN

END

C

SUBROUTINE DR2 (D, $C B, S B)$ 


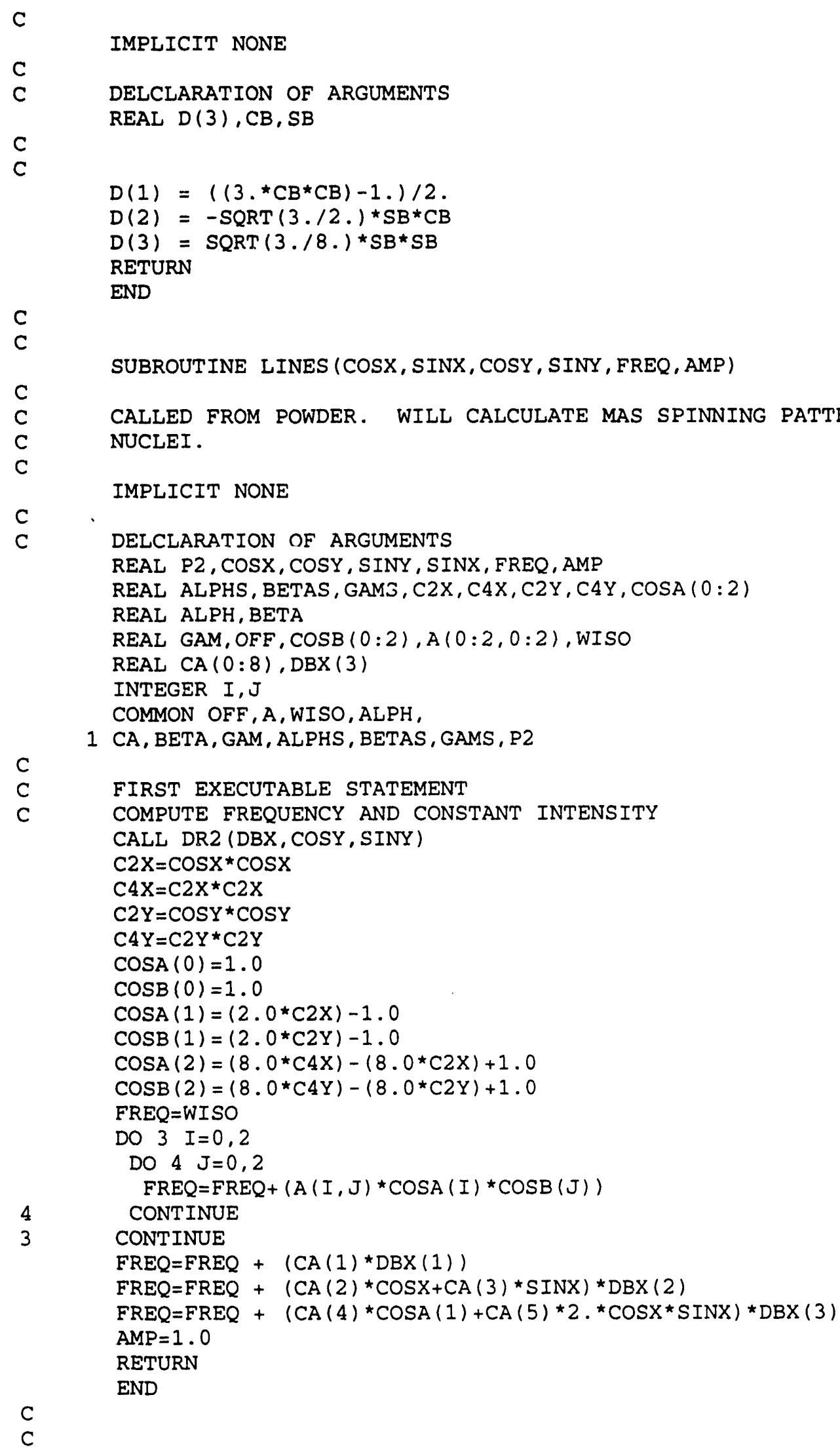


C

C

C

C

10

C

$\mathrm{C}$

C

C

C

C

C

C

C

C

C

C

C

C

C

SUBROUTINE JRAN (JRN, IDUM)

INTEGER M1, IA1, IC1, M2, IA2, IC2, M3, IA3, IC3

INTEGER IX1,IX2,IX3,J, IFF, IDUM

REAL RM1, RM2, JRN, TEMP, R (98)

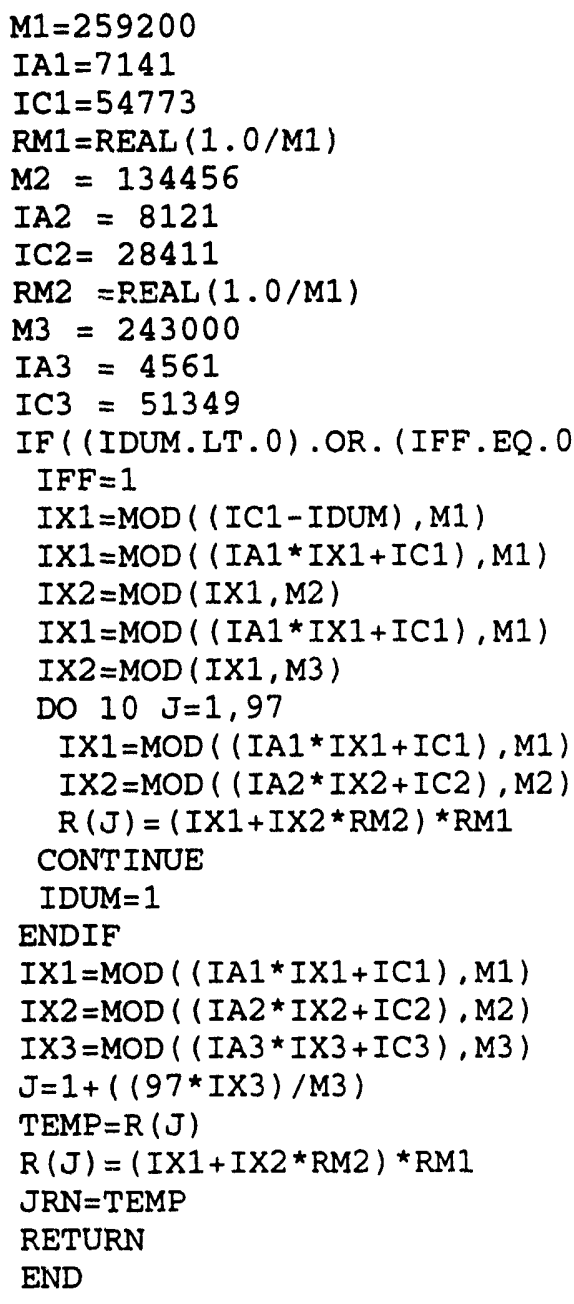


C

C

C

C

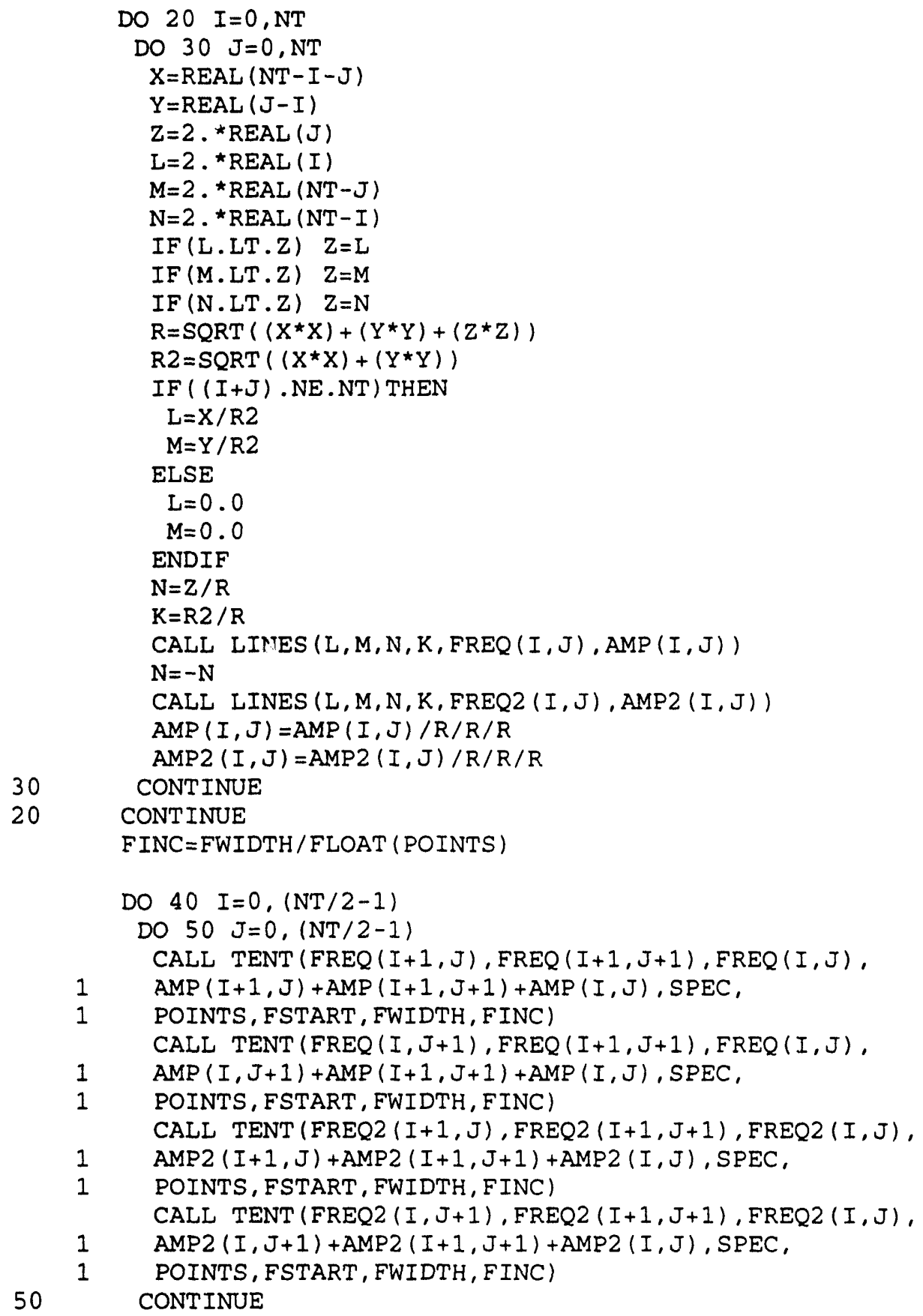


DO $41 \mathrm{I}=(\mathrm{NT} / 2),(\mathrm{NT}-1)$

DO $51 \mathrm{~J}=(\mathrm{NT} / 2),(\mathrm{NT}-1)$

CALL TENT (FREQ $(I+1, J), \operatorname{FREQ}(I+1, J+1), F R E Q(I, J)$,

1 $\operatorname{AMP}(I+1, J)+\operatorname{AMP}(I+1, J+1)+\operatorname{AMP}(I, J), \operatorname{SPEC}$,

1 POINTS, FSTART, FWIDTH, FINC)

CALL TENT (FREQ $(I, J+1), \operatorname{FREQ}(I+1, J+1), \operatorname{FREQ}(I, J)$, $\operatorname{AMP}(I, J+1)+\operatorname{AMP}(I+1, J+1)+\operatorname{AMP}(I, J), \operatorname{SPEC}$ POINTS, FSTART, FWIDTH, FINC)

CALL TENT (FREQ2 $(I+1, J), \operatorname{FREQ2}(I+1, J+1), \operatorname{FREQ2}(I, J)$, POINTS, FSTART, FWIDTH, FINC)

CALL TENT (FREQ2 (I, J + 1), FREQ2 (I + 1,J+1), FREQ2 (I, J),

DO $42 \mathrm{I}=0,(\mathrm{NT} / 2-1)$

DO $52 \mathrm{~J}=(\mathrm{NT} / 2),(\mathrm{NT}-1)$

CALL TENT (FREQ $(I+1, J), \operatorname{FREQ}(I, J+1), \operatorname{FREQ}(I, J)$,

$1 \operatorname{AMP}(I+1, J)+\operatorname{AMP}(I, J+1)+\operatorname{AMP}(I, J), S P E C$,

1 POINTS, FSTART, FWIDTH, FINC)

CALL TENT (FREQ $(I, J+1), F R E Q(I+1, J+1), F R E Q(I+1, J)$,

$1 \operatorname{AMP}(I, J+1)+\operatorname{AMP}(I+1, J+1)+\operatorname{AMP}(I+1, J), \operatorname{SPEC}$,

1 POINTS, FSTART, FWIDTH, FINC)

CALL TENT (FREQ2 $(I+1, J), \operatorname{FREQ2}(I, J+1), \operatorname{FREQ2}(I, J)$,

$1 \operatorname{AMP2}(I+1, J)+\operatorname{AMP} 2(I, J+1)+\operatorname{AMP} 2(I, J), \operatorname{SPEC}$,

1 POINTS, FSTART, FWIDTH, FINC)

CALL TENT (FREQ2 $(I, J+1), \operatorname{FREQ2}(I+1, J+1), \operatorname{FREQ2}(I+1, J)$,

$1 \operatorname{AMP} 2(I, J+1)+\operatorname{AMP} 2(I+1, J+1)+\operatorname{AMP} 2(I+1, J), \operatorname{SPEC}$,

1 POINTS, FSTART, FWIDTH, FINC) CONTINUE

CONTINUE

DO $43 I=(\mathrm{NT} / 2),(\mathrm{NT}-1)$

DO $53 \mathrm{~J}=0,(\mathrm{NT} / 2-1)$

CALL TENT (FREQ $(I+1, J), \operatorname{FREQ}(I+1, J+1), \operatorname{FREQ}(I, J)$,

$1 \operatorname{AMP}(I+1, J)+\operatorname{AMP}(I+1, J+1)+\operatorname{AMP}(I, J), \operatorname{SPEC}$,

1 POINTS, FSTART, FWIDTH, FINC)

CALL TENT (FREQ $(I, J+1), F R E Q(I+1, J+1), F R E Q(I, J)$,

$1 \operatorname{AMP}(I, J+1)+\operatorname{AMP}(I+1, J+1)+\operatorname{AMP}(I, J), \operatorname{SPEC}$,

1 POINTS, FSTART, FWIDTH, FINC)

CALL TENT (FREQ2 $(I+1, J), F R E Q 2(I, J+1), F R E Q 2(I, J)$,

$1 \operatorname{AMP} 2(I+1, J)+\operatorname{AMP} 2(I, J+1)+\operatorname{AMP} 2(I, J), S P E C$,

1 POINTS, FSTART, FWIDTH, FINC)

CALL TENT (FREQ2 ( I J J 1), FREQ2 (I+1,J+1), FREQ2 (I+1,J),

$1 \operatorname{AMP} 2(I, J+1)+\operatorname{AMP} 2(I+1, J+1)+\operatorname{AMP} 2(I+1, J), \operatorname{SPEC}$.

1 POINTS, FSTART, FWIDTH, FINC)

CONTINUE

43 CONTINUE

RETURN

END

C

C

C 


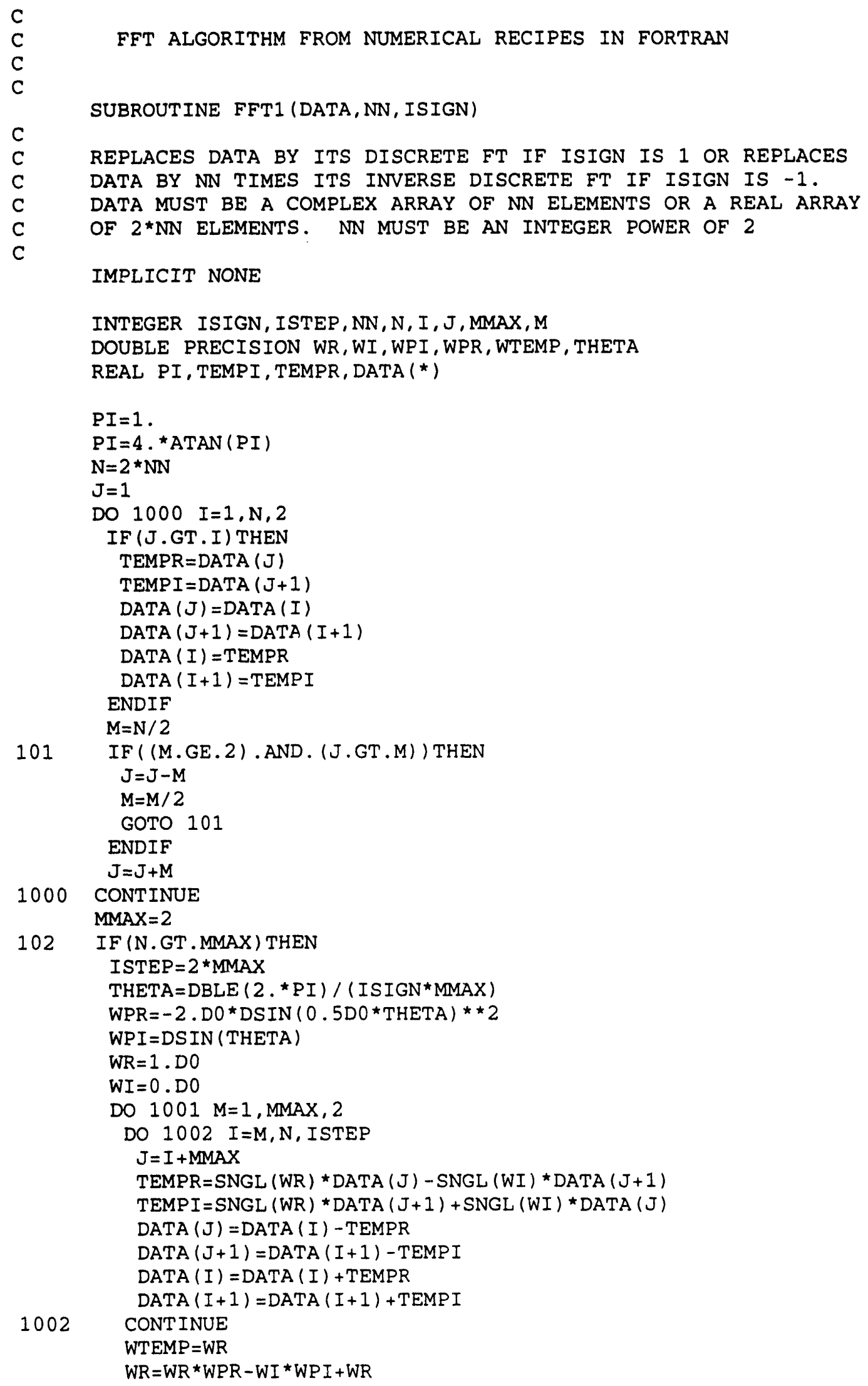

C

C

C

SUBROUTINE FFT1 (DATA, NN, ISIGN)

C

C

C

C

C

REPLACES DATA BY ITS DISCRETE FT IF ISIGN IS 1 OR REPLACES DATA BY NN TIMES ITS INVERSE DISCRETE FT IF ISIGN IS -1 . DATA MUST BE A COMPLEX ARRAY OF NN ELEMENTS OR A REAL ARRAY OF 2*NN ELEMENTS. NN MUST BE AN INTEGER POWER OF 2

IMPLICIT NONE

INTEGER ISIGN, ISTEP, NN, $N, I, J, M M A X, M$

DOUBLE PRECISION WR, WI, WPI, WPR, WTEMP, THETA

REAL PI, TEMPI, TEMPR, DATA (*) 
$W I=W I$ * WPR $+W T E M P$ * WPI $+W I$

CONT INUE

MMAX = ISTEP

GOTO 102

ENDIF

RETURN

END

C

C

C

1

TENT ALGORITHM FROM ALDERMAN ET. AL. IN POWDER PROGRAM

SUBROUTINE TENT (FREQ1, FREQ2, FREQ3, AMP , SPEC, POINTS,

1 FSTART, FWIDTH, FINC)

CALLED FROM POWDER. ADDS TO SPECTRUM THE "TENT"

WHICH REPRESENTS THE

CONTRIBUTION FROM A TRIANGLE ON THE VERTICES OF WHICH THE

FREQUENCIES ARE FREQ1, FREQ2, FREQ3.

IMPLICIT NONE

$\mathrm{C}$

C DECLARATION OF ARGUMENT VARIABLES.

REAL FREQ1, FREQ2, FREQ3, AMP

INTEGER POINTS

REAL SPEC (0:POINTS-1), FSTART, FWIDTH, FINC

C

C DECLARATION OF INTERNAL VARIABLES.

REAL AREA3, AREA1, AREA2, FMIN, FMID, FMAX, F1, F2, TOP

INTEGER P, PMID, PMAX

C

FIRST EXECUTABLE STATEMENT.

SORT THE FREQUENCIES

FMIN=AMIN1 (FREQ1, FREQ2, FREQ3)

FMID=AMIN1 (AMAX1 (FREQ1, FREQ2), AMAX1 (FREQ2, FREQ3) ,

1 AMAX1 (FREQ3, FREQ1))

FMAX=AMAX1 (FREQ1, FREQ2, FREQ3)

C COMPUTE HEIGHT OF "TENT".

IF (FMAX . NE . FMIN) TOP $=A M P \star 2.0 /$ (FMAX -FMIN)

C COMPUTE INDICES OF TENT EDGES AND TOP

$P=I N T($ (FMIN-FSTART) /FINC)

PMID $=$ INT $($ (FMID-FSTART $) / F I N C)$

PMAX $=$ INT $($ (FMAX -FSTART $) / F I N C)$

C LOOK FOR CONTRIBUTIONS OUTSIDE OF SPECTRUM.

IF (PMAX.GE.POINTS) PMAX=POINTS

IF (PMID.GE.POINTS) PMID=POINTS

IF (P.GE.POINTS) $P=$ POINTS

IF (P.LT.O) $\mathrm{P}=0$

IF (PMID.LT.0) PMID $=0$

IF (PMAX.LT.0) PMAX $=0$

AREA $1=$ TOP $/(2 . *($ FMID-FMIN $))$

AREA $2=$ TOP $/(2 . *($ FMAX - FMID $))$

AREA3 $=$ TOP $/ 2$.

C ERECT "TENT" BY EXAMINING VARIOUS CASES.

IF (P.NE.PMID) GO TO 10

$\operatorname{SPEC}(P)=\operatorname{SPEC}(\mathrm{P})+($ FMID - FMIN $)$ *AREA 3

GO TO 40

$10 \quad$ F2 $=$ FINC * REAL $(P+1)+$ FSTART

$\operatorname{SPEC}(P)=\operatorname{SPEC}(P)$ 
$1+($ F2 $-F M I N)$ * $(F 2-F M I N) * A R E A 1$

$\mathrm{P}=\mathrm{P}+1$

$F 1=F 2$

IF (P.EQ.PMID) GO TO 30

$F 2=F 2+F I N C$

$\operatorname{SPEC}(P)=\operatorname{SPEC}(P)$

1 +FINC* $(F 2+F 1-2$ *FMIN $)$ *AREAI

GO TO 20

$30 \quad \operatorname{SPEC}(P)=\operatorname{SPEC}(P)$

$1+(F M I D-F 1) *(F M I D+F 1-2$ *FMIN $)$ *AREA1

40 IF (P.NE.PMAX) GO TO 50

$\operatorname{SPEC}(P)=\operatorname{SPEC}(P)+($ FMAX - FMID $)$ *AREA 3

GO TO 80

50 F2 =FINC *REAL $($ PMID+1)+FSTART

$\operatorname{SPEC}(P)=\operatorname{SPEC}(P)$

60

$1+(F 2-F M I D) *(2$ *FMAX $-F 2-F M I D)$ * AREA2

$\mathrm{P}=\mathrm{P}+1$

$F 1=F 2$

IF (P.EQ.PMAX) GO TO 70

$F 2=F 2+F I N C$

$\operatorname{SPEC}(P)=\operatorname{SPEC}(P)$

$1+F I N C$ * $(2$ * FMAX $-F 1-F 2)$ *AREA2

GO TO 60

$70 \quad \operatorname{SPEC}(P)=\operatorname{SPEC}(\mathrm{P})$

80 CONTINUE

RETURN

END

\section{MINUITCQ - VAS Least Squares Fitting Program}

MINUITCQ uses many of the same subroutines as the previous CQP program. In all cases these are the same, except where indicated by specific inclusion in the program below. The code for the MINUIT subroutine is not included here but may be acquired from CERN. This is a very powerful minimization library which is applicable to a wide range of programming needs. Both MINUITCQ and CQP are written in the usual FORTRAN-77 with no extensions. This code may be acquired from the author of this thesis or Prof. A. Pines at the University of California, Berkeley.

$\begin{array}{lc}\text { C } & \text { THIS PROGRAM WAS WRITTEN BY JAY BALTISBERGER } \\ \text { C } & \text { PINES RESEARCH GROUP } \\ \text { C } & \text { UCBERKELEY } \\ \text { C } & \text { BERKELEY, CA } 94720 \\ \text { C } & \\ \text { C } & \text { THIS WILI CALCULATE AND FIT A QUADRUPOLAR LINESHAPE SPINNING } \\ \text { C } & \text { ABOUT ANY AXIS. (WITH O DEGREES BEING EQUIVALENT TO STATIC) } \\ \text { C } & \text { THE ASSUMPTION IS THAT THE SPINNING RATE IS GREATER THAN THE } \\ \text { C } & \text { OVERALL WIDTH OF THE PATTERN SO NO SIDEBANDS ARE INCLUDED. }\end{array}$


ALSO IN ADDITION TO THE 2ND ORDER QUADRUPOLAR LINESHAPE, WE MAY INCLUDE A CSA WITH AXES NON-COINCIDENT WITH THE QUADRUPOLAR AXES. LINE BROADENING IS INCLUDED BOTH WITH GAUSSIAN AND LORENTZIAN COMPONENTS. TO RUN THIS PROGRAM ON A UNIX BASED MACHINE, YOU MUST COMPILE AND LINK THIS AS BEFORE.

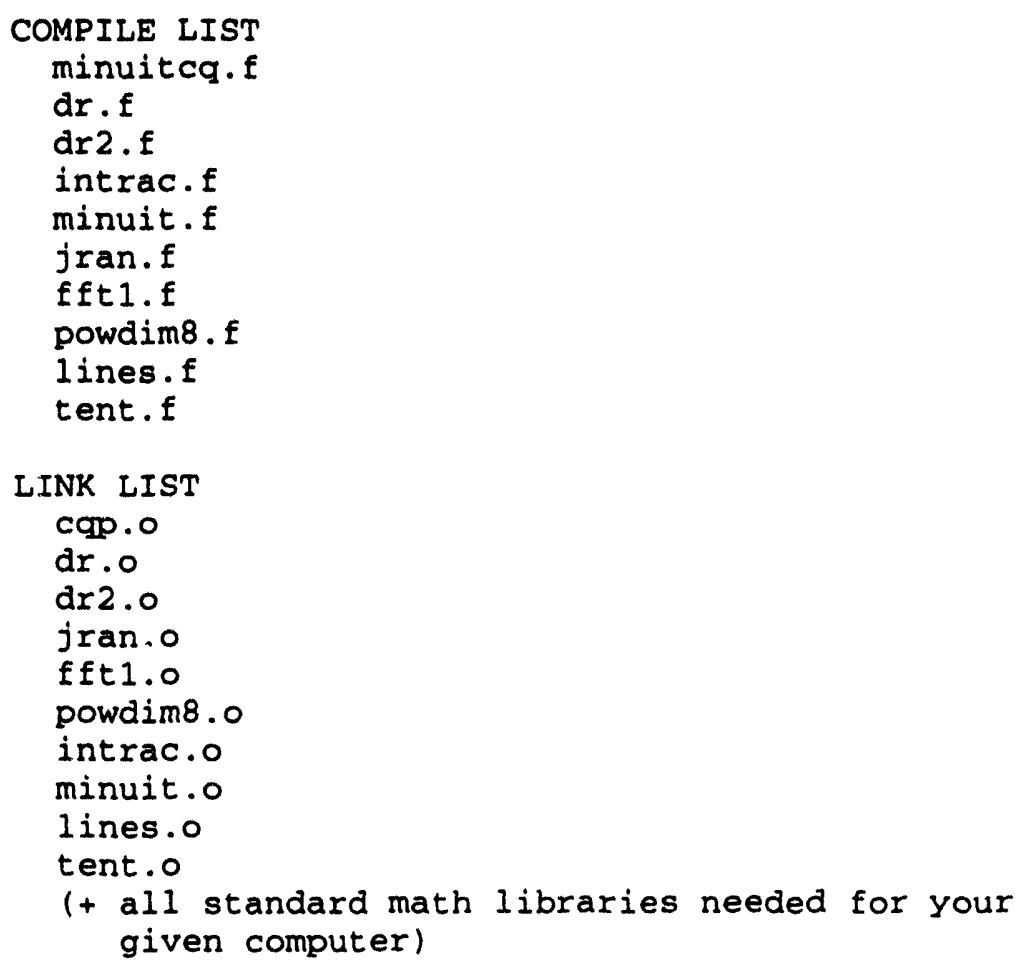

THE BASIS OF THIS PROGRAM COMES FROM A SIMULATION CODE WRITTEN BY ALDERMAN, GRANT, ET. AL. AT U.OF UTAH, JUST LIKE CQP.F ALSO CSA/QUAD COMBINED FORMULA APPEAR THROUGHOUT THE LITERATURE SUCH AS THE PAPER BY BAUGHER, BRAY, ET. AL.

IN ADDITION THE NONCOINCIDENT AXES HAS BEEN DESCRIBED MANY

TIMES AS WELL SUCH AS BY P. ELLIS, ET. AL. AT U OF S.CAR.

FUNCTION DUMMY $(\mathrm{X})$

REAL DUMMY, $X$

$D U M M Y=X$

RETURN

END

$\mathrm{C}$

C

FUNCTION SQAVELEVEL (ITER)

IMPLICIT NONE

C

DECIAARATION OF VARIABLES

REAL SQAVELEVEL, F'WIDTH, FSTART

INTEGER START, MM, SHOW, COUNT, NTRAN, QPTE, CSTE, SPIN, ITER, PATS, I

REAL WISO, WL, P2

REAL P4

REAL ZERO, FINC, SPECIN $(0: 1023)$ 
REAL $A(0: 2,0: 2)$, VAL , OFF

REAL PARAM $(0: 32,0: 64,5)$, CHIFIX, CA $(0: 8)$

COMMON SPECIN, A, WISO, PATS, SPIN, WL , P2, P4 , OFF, FWIDTH, FSTART, FINC

C

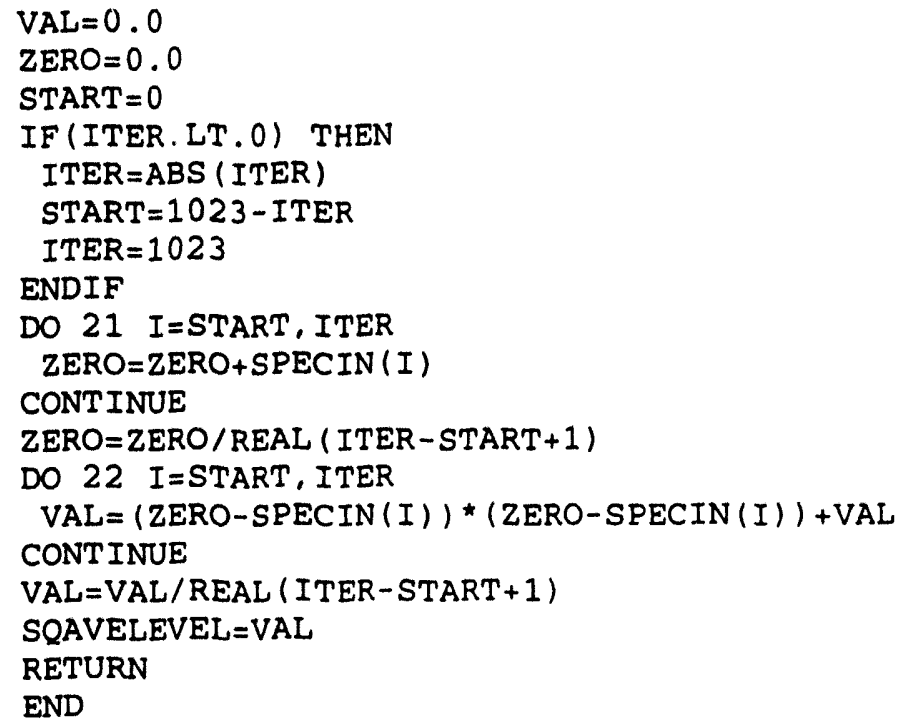

C

C

SUBROUTINE FXT (NPAR, GRAD, FCT, PR, IFLAG, DUMMY) PROGRAM WRITTEN TO CALCULATE MULTIPLE POWDER PATTERNS GIVEN THE QUADRUPOLAR PARAMETERS OF EACH.

IN ORDER TO SEE THE RESULT THE USER MUST PROVIDE A SUBROUTINE SHOW(SPEC, POINTS) WHICH DISPLAYS THE CALCULATED SPECTRUM ON WHATEVER DEVICE IS AVAILABLE. THE ARGUMENT SPEC IS A REAL ARRAY WITH POINTS ELEMENTS. THE LARGEST VALUE IN THE SPECTRUM IS UNITY.

EXTERNAL DUMMY

SIZE OF SPECTRUM

DECLARATION OF VARIABLES

INTEGER SIZE, NPAP, IFLAG

PARAMETER (SIZE=1024)

REAL GRAD $(60)$, DUMMY

REAL SPEC (0:SIZE-1),DATA (1:2*SIZE),DATA2 (1:2*SIZE)

REAL FCT, PR (60), FWIDTH, AMP, MAXIMUM, FSTART

INTEGER MM, COUNT, NUM, QPTE, CSTE, PATS, I, J , POINTS, P, ISIGN

INTEGER SHOW, NTRAN

REAI, SPIN, ERROR, ETA, WISO, PI, WL, WQ , C, P2

REAL DECAY, P4, DBETA (7), AL2GAM2, NAL2GAM2, SNAL2GAM2, SAL2GAM2

REAL AL2GAM, AL2NGAM, SAL2GAM, SAL2NGAM

REAL FINC, ETA2, SPECIN $(0: 1023)$, BROADL, BROADG

REAL EPLG, A $(0: 2,0: 2)$, GOBBLE, OFF

REAL PARAM $(0: 32, C: 64,5), \operatorname{CHIFIX,CA}(0: 8)$

COMMON SPECIN, A, WISO, PATS, SPIN, WL, P2, P4, OFF, FWIDTH, FSTART, FINC

C

,MM, SHOW, COUNT, QPTE, CSTE, PARAM, NTRAN, CHIFIX, CA

FIRST EXECUTABLE STATEMENT. 


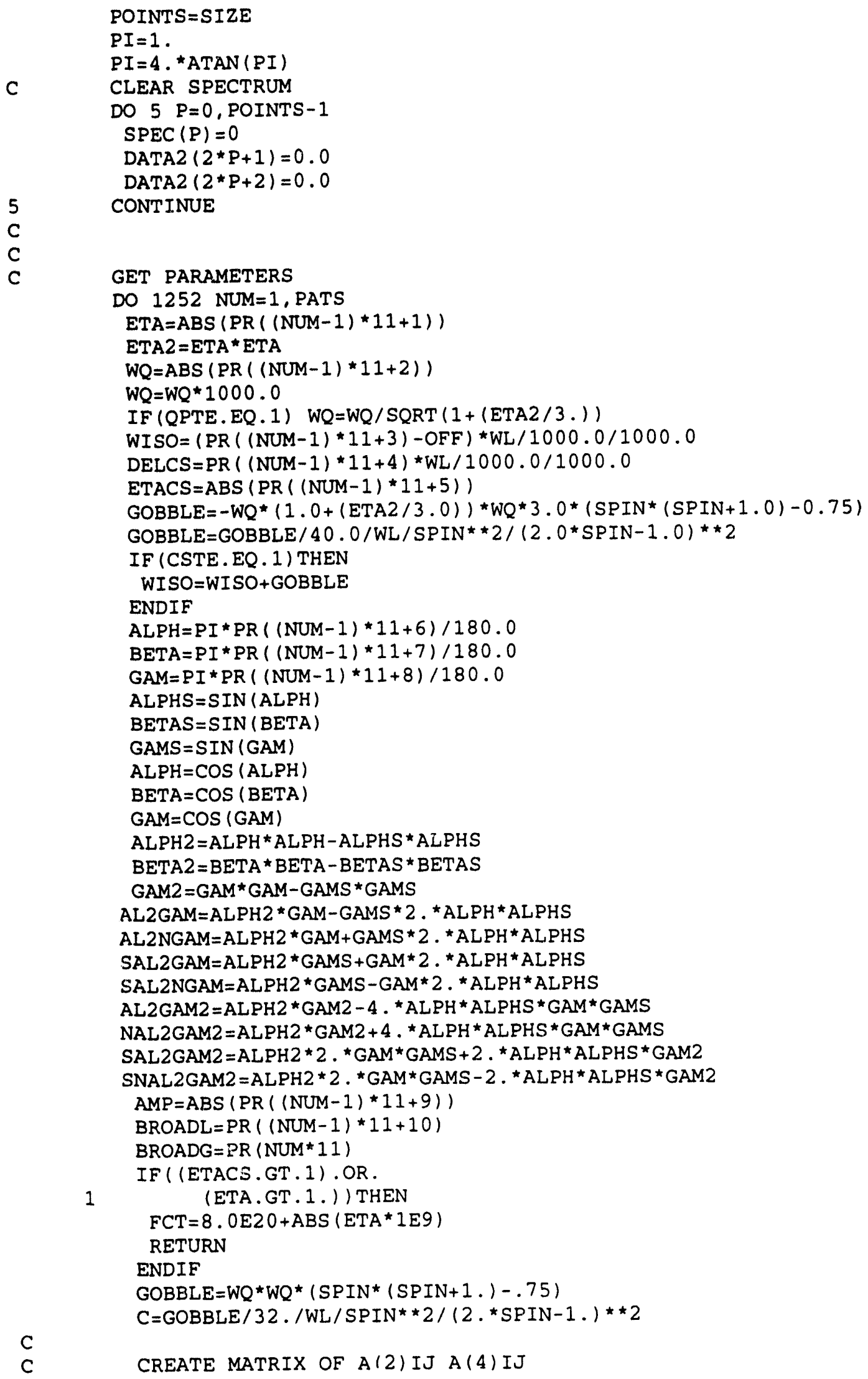




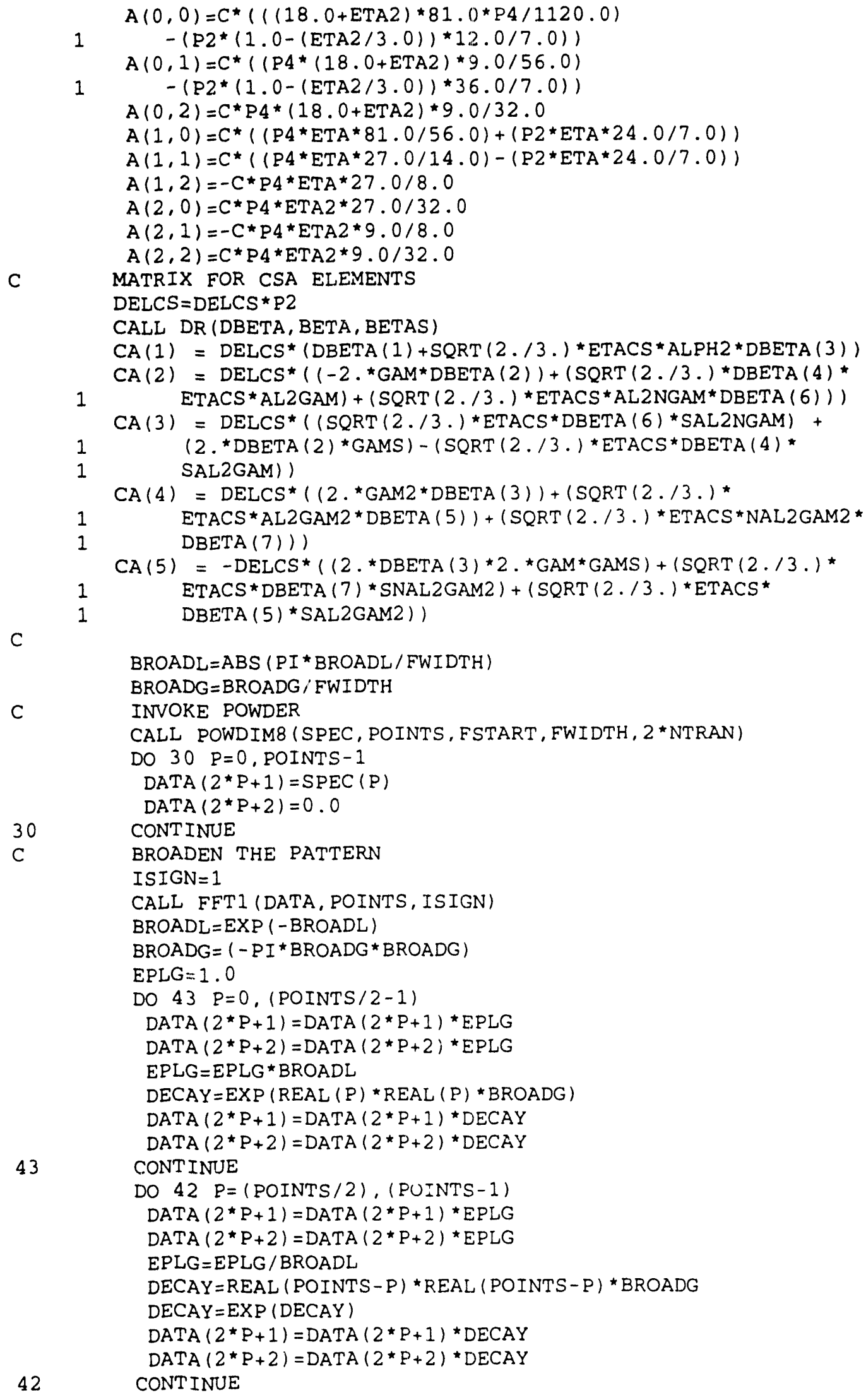




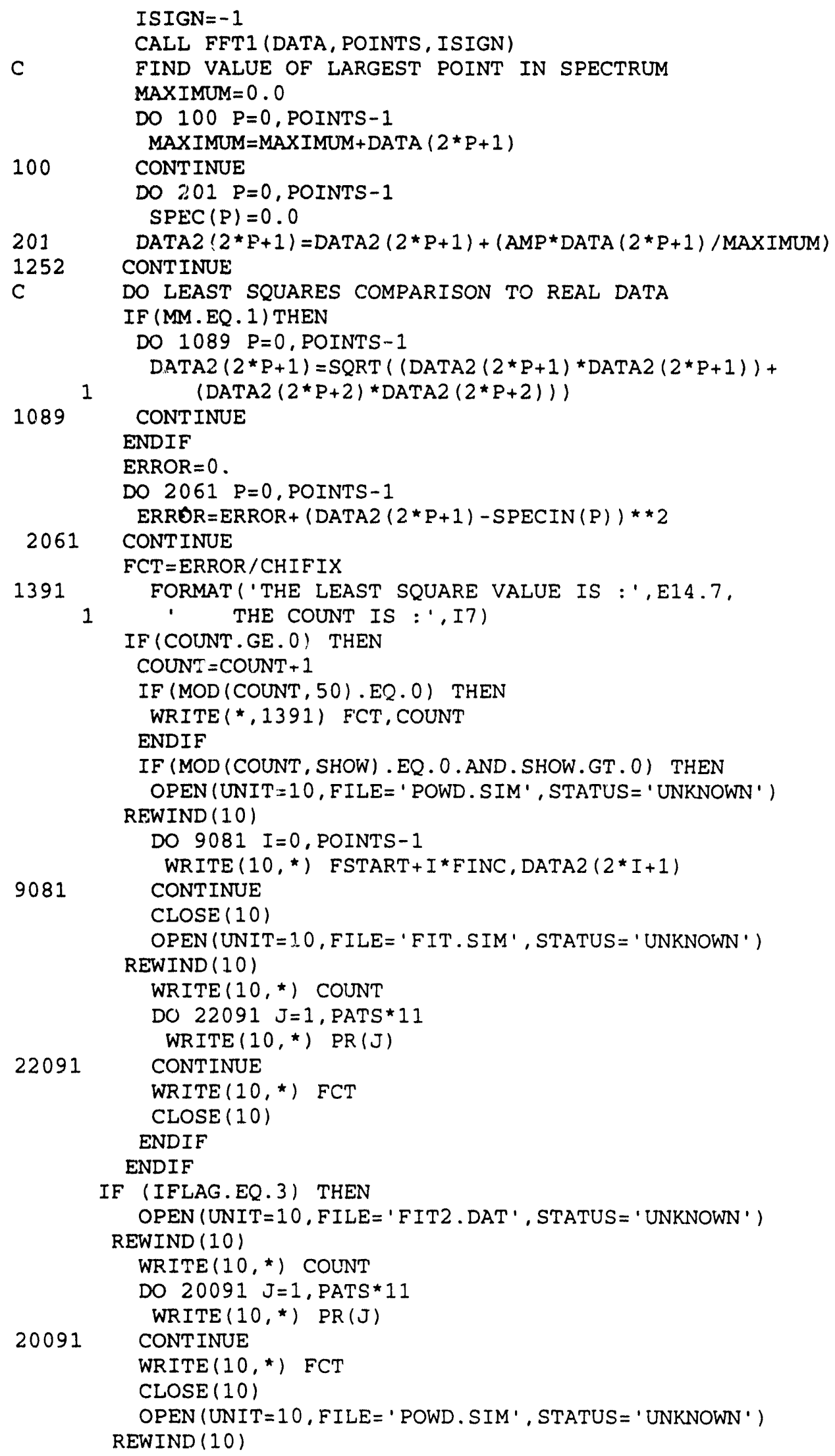




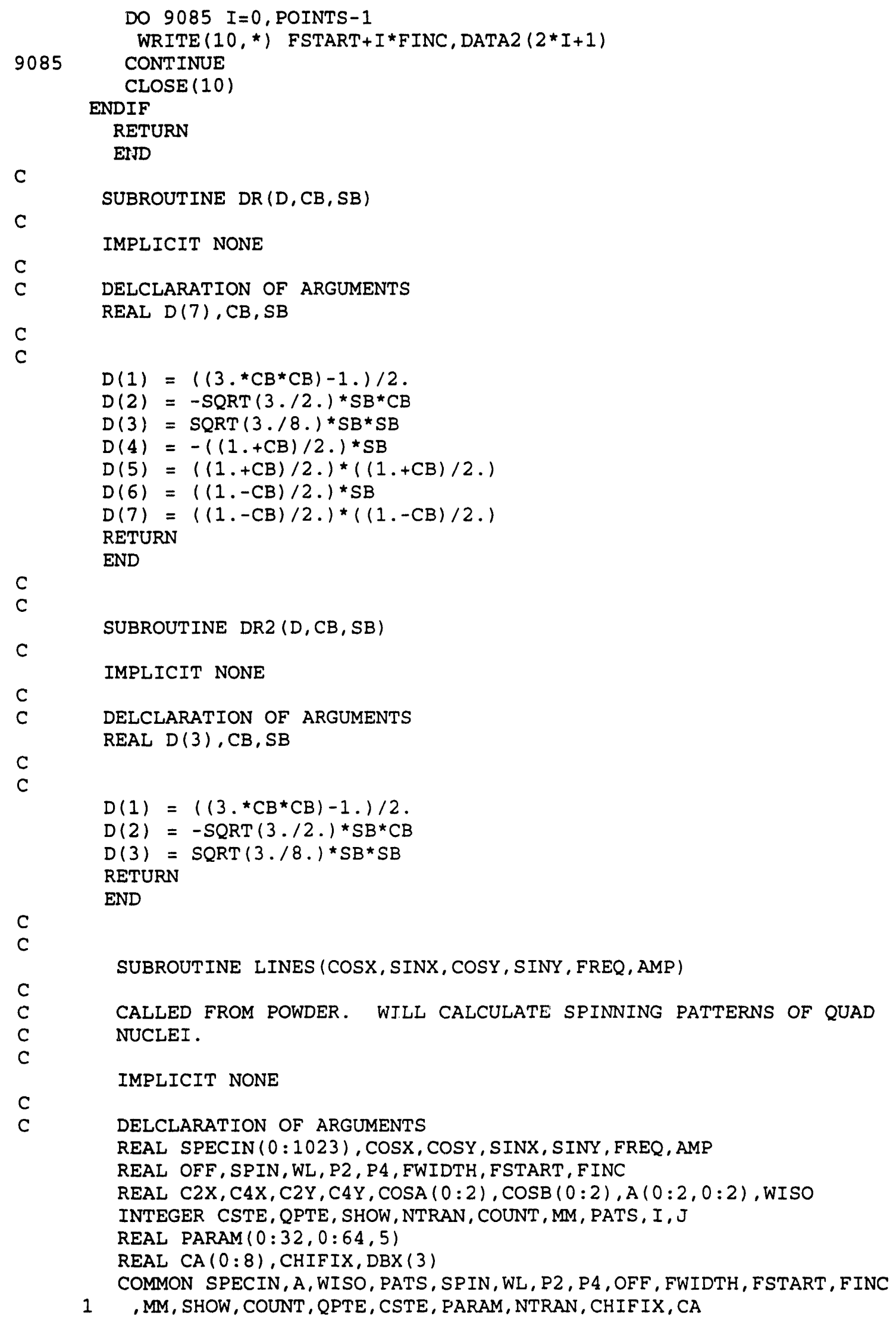


C

C

PROGRAM MINUITCQ

EXTERNAL FXT

EXTERNAL DUMMY

INTEGER QPTE, CSTE, NTRAN

INTEGER MM, SHOW, COUNT, I, PATS, POINTS

CHARACTER * 30 FILENM

CHARACTER $* 1$ ANSWER

REAL OFF

REAL SPECIN $(0: 1023), A(0: 2,0: 2)$, WISO

REAL WL, THETA, PI, SPIN, SW, C2, C4, P2, P4, FWIDTH, FSTART, FINC

REAL PARAM $(0: 32,0: 64,5)$, CHIFIX, CA $(0: 8)$

COMMON SPECIN, A, WISO, PATS, SPIN, WL, P2, P4, OFF, FWIDTH, FSTART, FINC

1 , MM, SHOW, COUNT, QPTE, CSTE, PARAM, NTRAN, CHIFIX, CA 


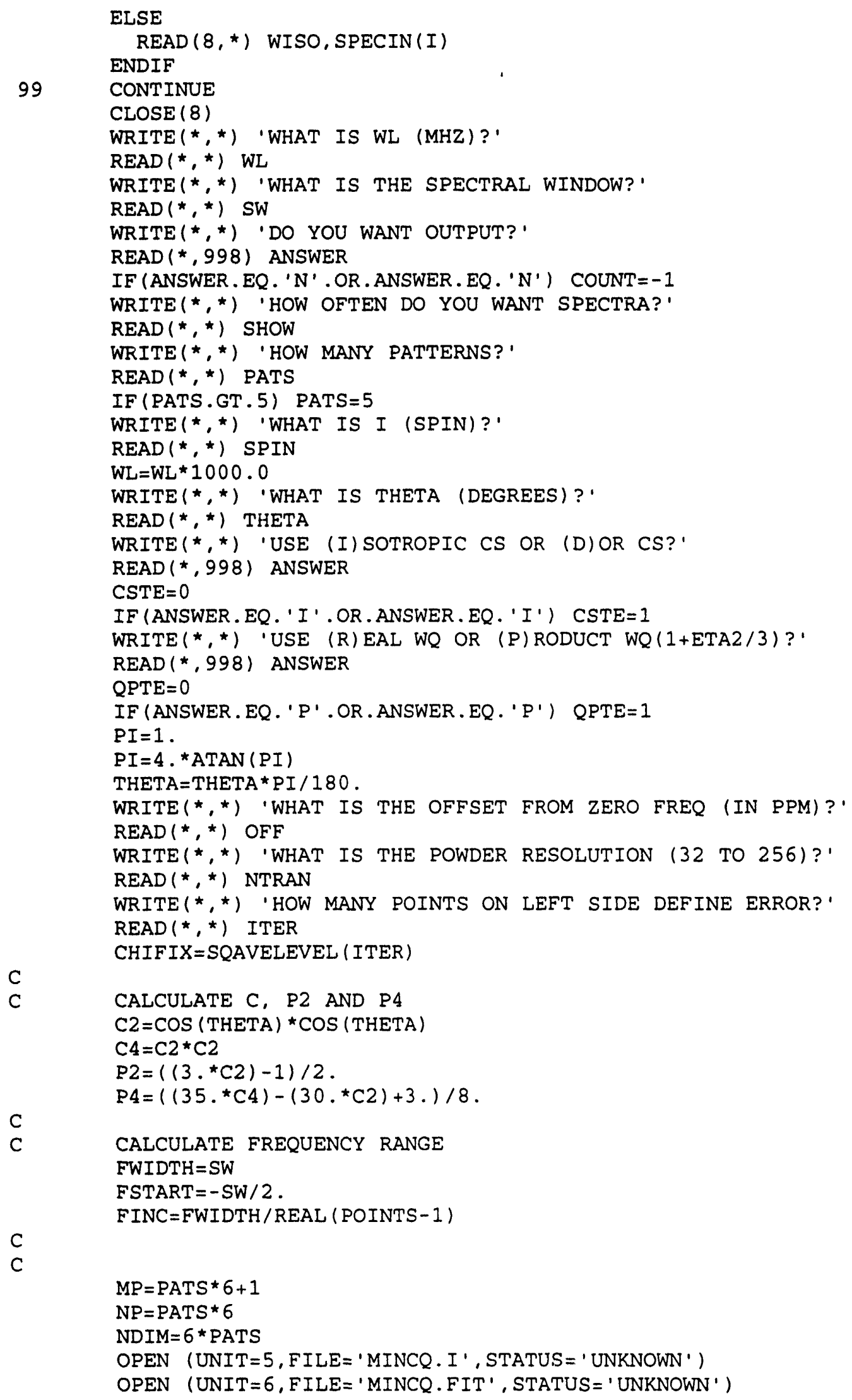


CALL MINUIT (FXT, DUMMY)

CLOSE (5)

CLOSE (6)

END

C

C

FUNCTION INTRAC

LOGICAL * 4 INTRAC

INTRAC $=$.FALSE.

RETURN

C

END 

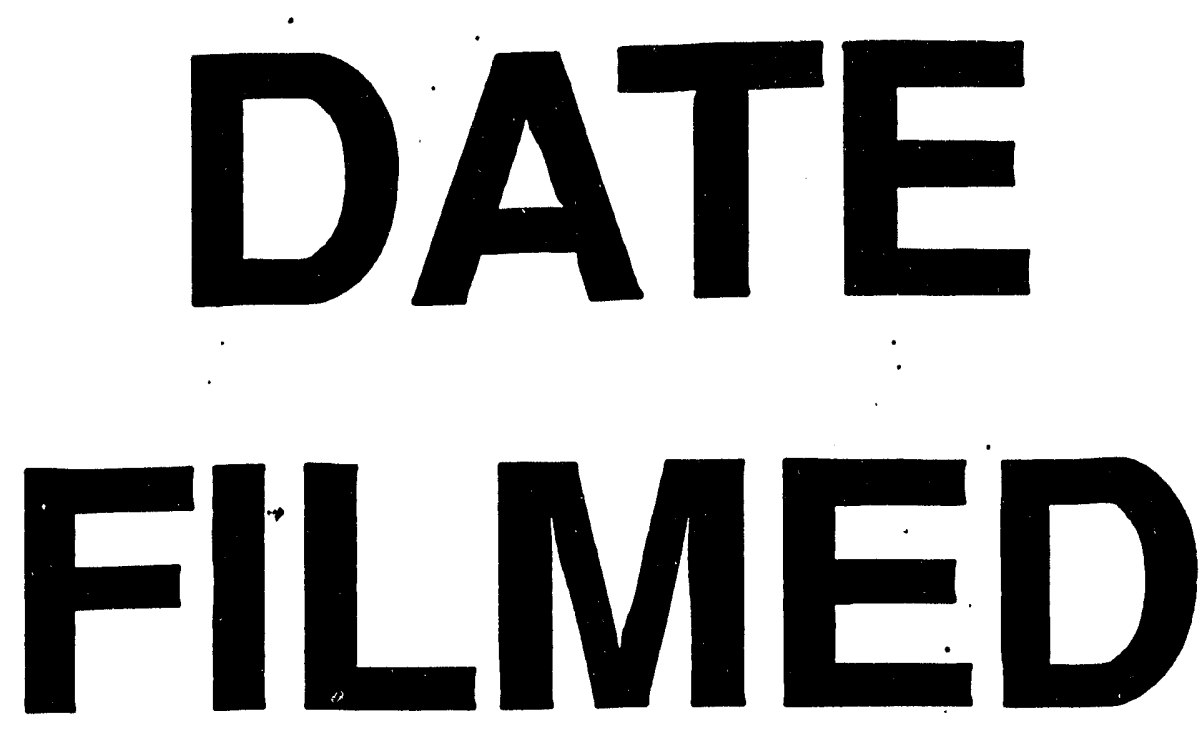

$3 / 23 / 94$
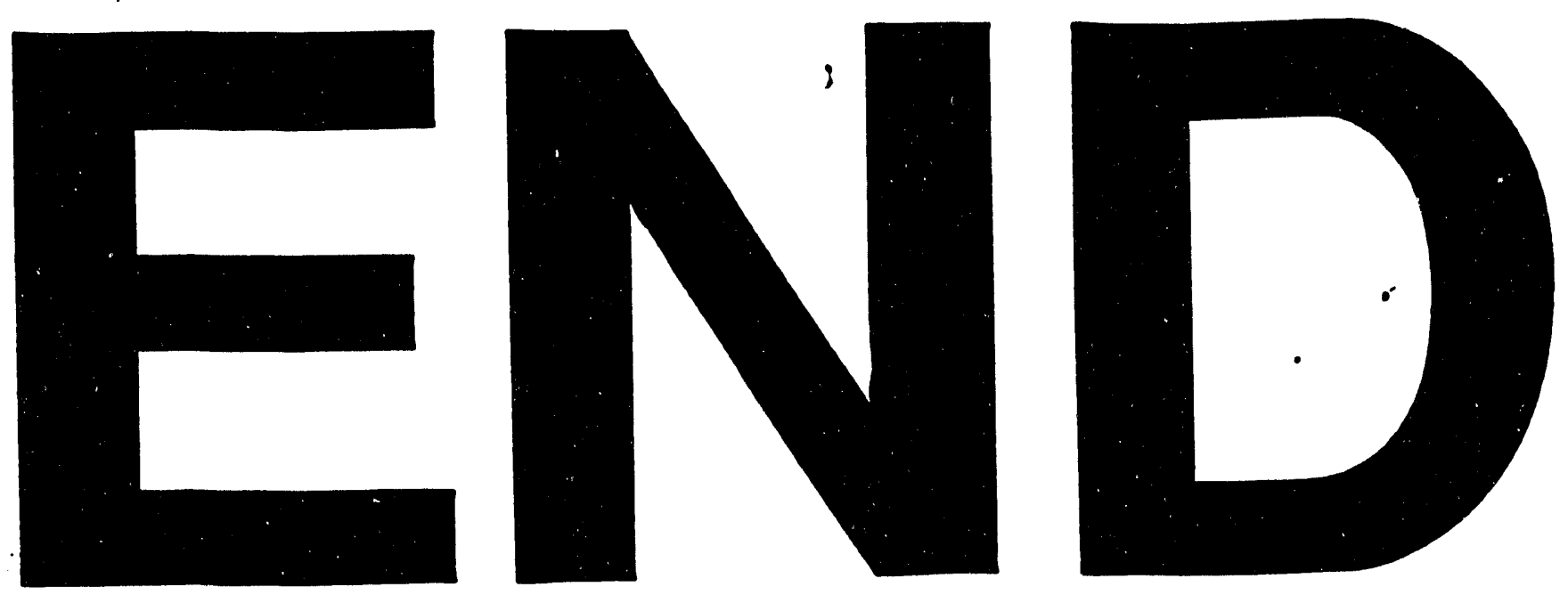
